\title{
Understanding the Torquoselectivity in $8 \pi$-Electrocyclic Cascade Reactions: Synthesis of Fenestradienes versus Cyclooctatrienes
}

\author{
Catherine Hulot, ${ }^{\ddagger}$ Shadi Amiri, ${ }^{\S}$ Gaëlle Blond, ${ }^{\ddagger}$ Peter R. Schreiner,${ }^{*}$, and Jean Suffert ${ }^{*, *}$
}

Faculté de Pharmacie, Université de Strasbourg (UMR 7200 CNRS/UDS), 74 Route du Rhin, 67401 Illkirch/Strasbourg, France; Institut für Organische Chemie, Justus-Liebig-Universität Giessen, Heinrich-Buff-Ring 58, D-35392 Giessen, Germany

* Université de Strasbourg

$\S$ Justus-Liebig-Universität Giessen

\section{Supporting Information}

\section{Contents}

General Methods: S1

Characterization data for 5-34: S2-S16

Computational Section : S17-S80

References of Supporting Information : S80

Then NMR spectra

General Methods. Reactions were run under an atmosphere of argon in oven-dried glassware using a standard syringue and septa apparatus. $\mathrm{Et}_{2} \mathrm{O}$ and THF were distilled from sodium / benzophenone. Crude products were purified by flash column chromatography on Merck silica gel Si 60 (40-63 $\mu \mathrm{m})$. For some compounds, $5 \% \mathrm{Et}_{3} \mathrm{~N}$ treated silica gel was used to avoid decomposition. Analytical TLC was carried out on Merck aluminium sheets silica gel $60 \mathrm{~F}_{254} \cdot{ }^{1} \mathrm{H}$ NMR spectra were recorded at 200,300 or $500 \mathrm{MHz}$ using the residual solvent signal as internal reference $\left(\mathrm{CDCl}_{3}, 7.26 \mathrm{ppm}\right)$. Chemical shifts are quoten in ppm, coupling constants $(J)$ are given in Hz. The following abbreviations are used to describe peak patterns when appropriate: $\mathrm{s}$ (singlet), $\mathrm{d}$ (doublet), $\mathrm{t}$ (triplet), q (quartet), quint (quintuplet), m (multiplet), ap (apparent), br (broad). ${ }^{13} \mathrm{C}$ NMR spectra were recorded at 50 or $75 \mathrm{MHz}$ at ambient temperature in $\mathrm{CDCl}_{3}$ with $\delta\left(\mathrm{CDCl}_{3}\right)$ at 77.16 as internal standard. Multiplicities were determined in some cases by DEPT pulse sequence and are given as follow: $+=\mathrm{CH}$ or $\mathrm{CH}_{3}$, $-=\mathrm{CH}_{2}$, and $\mathrm{C}_{\text {quat }}=\mathrm{C}$. Melting points were determined with a glass capillary apparatus Gallenkamp and are uncorrected. I.R were determined with a Nicolet 380 FT-IR apparatus. We thank Pr. Van Dorsselaer (LSMBO, Cronenbourg, France) to lend us the spectrometers for mass spectra. Gas Chromatography Mass Spectrometry (GCMS) were performed using an Agilent 5975 GCMS spectrometer (EI Quad, positive ion $70 \mathrm{eV}$ ). High Resolution Mass Spectral analysis (HRMS) were performed using a Bruker MicroTOF-Q (ESI Q-TOF, positive ion: solution of lithium formiate in isopropanol, $10 \mathrm{mM}$ ). $n$-BuLi was titrated using $N$-pivaloyl $o$ toluidine following the Suffert's procedure. ${ }^{1}$ X-ray Cristallographic Study were done by the X-Ray Central Service of the Chemistry Institute of Strasbourg (France). Microwave irradiations have been performed using BIOTAGE Smith Creator. The program NameiPro (ACD/Name version 5.5.5.5) was used to name the different compounds.

Compounds $1,2,3,4,6,13,14,21,25,26,28,30,32$ have already been described. ${ }^{2}$ 


\section{- Synthesis of trienynes 5a-f}

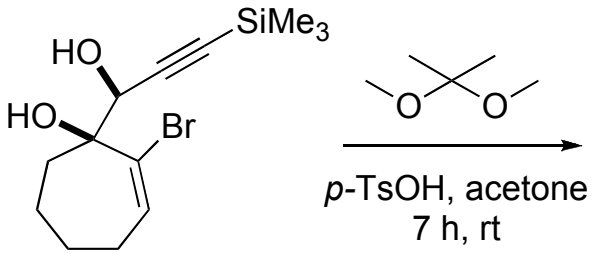

3

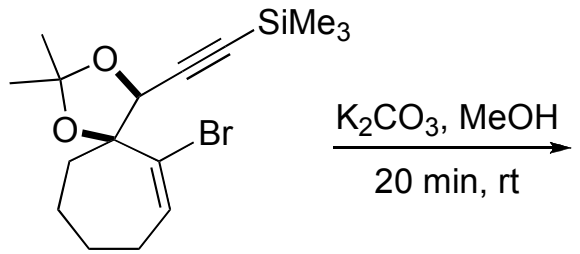

34

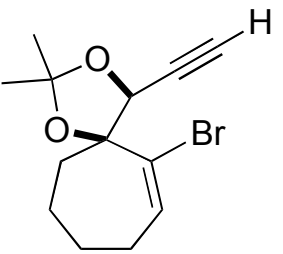

27
1) $n$-BuLi, $-78^{\circ} \mathrm{C}, 30 \mathrm{~min}$
2) $\left(\mathrm{CH}_{2} \mathrm{O}\right)_{\mathrm{n}},-78{ }^{\circ} \mathrm{C}, 1 \mathrm{~h}$ $18 \mathrm{~h}, \mathrm{rt}$

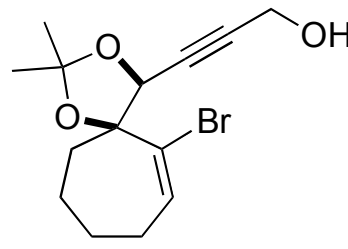

29

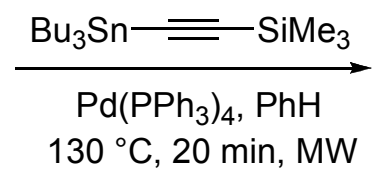

$130{ }^{\circ} \mathrm{C}, 20 \mathrm{~min}, \mathrm{MW}$

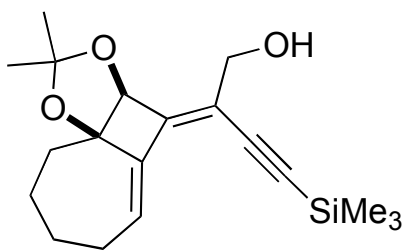

31

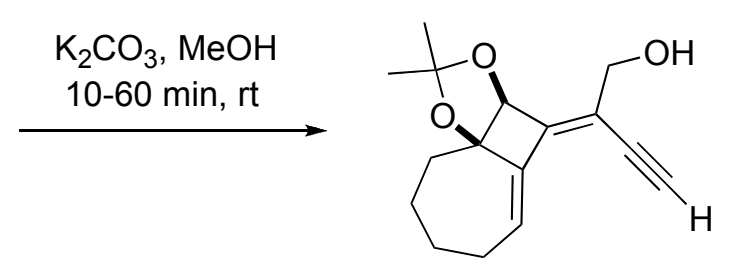

33

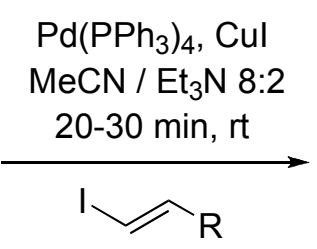

$\mathrm{MeCN} / \mathrm{Et}_{3} \mathrm{~N} 8: 2$

20-30 min, r

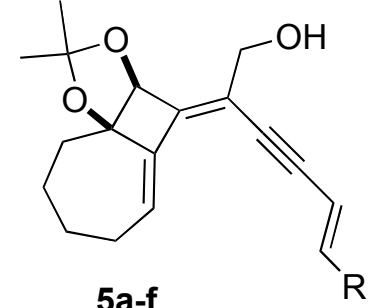

5a-f

$\left\{\left[\left(4 S^{*}, 5 S^{*}\right)-6-b r o m o-2,2-d i m e t h y l-1,3-d i o x a s p i r o[4.6]\right.\right.$ undec-6-en-4-yl]ethynyl $\}$ (trimethyl) silane (34)

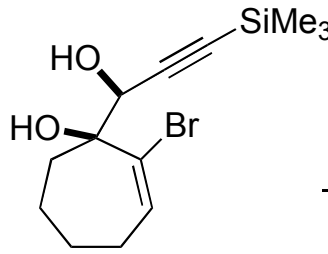

3

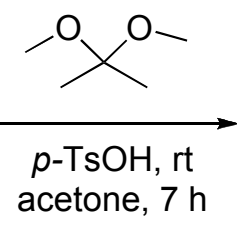

acetone, $7 \mathrm{~h}$

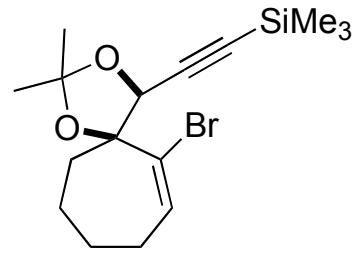

34

A solution of diol anti 3 (2 g, $6.30 \mathrm{mmol}, 1 \mathrm{eq}), 2$,2-dimethoxypropane (20 mL, $162.8 \mathrm{mmol}, 26$ eq), and $p$-TsOH $(200 \mathrm{mg}, 1.05 \mathrm{mmol}, 0.17 \mathrm{eq})$ in acetone $(50 \mathrm{~mL})$ was stirred for $7 \mathrm{~h}$ at room temperature. Then the mixture was quenched by addition of a saturated solution of $\mathrm{NaHCO}_{3}(50$ $\mathrm{mL})$. After extraction with $\mathrm{Et}_{2} \mathrm{O}$, the combined organic layers were washed with water, brine, dried over $\mathrm{MgSO}_{4}$ and concentrated in vacuo. The crude product was purified by chromatography on silica gel (elution with pentane/ $\left.\mathrm{Et}_{2} \mathrm{O} 98: 2\right)$ to give $2.1 \mathrm{~g}(93 \%)$ of the product as a white powder.

$\mathrm{C}_{16} \mathrm{H}_{25} \mathrm{BrO}_{2} \mathrm{Si}, \mathrm{MW}=357.36$ g.mol ${ }^{-1}$

$\mathbf{R f}=0.16\left(\right.$ Pentane $\left./ \mathrm{Et}_{2} \mathrm{O} 98: 2\right)$

$\mathbf{M p}=44^{\circ} \mathrm{C}$

${ }^{1}$ H NMR $\left(200 \mathrm{MHz}, \mathrm{CDCl}_{3}\right) \delta 6.54(\mathrm{t}, J=6.8 \mathrm{~Hz}, 1 \mathrm{H}), 4.82(\mathrm{~s}, 1 \mathrm{H}), 2.28-1.58(\mathrm{~m}, 8 \mathrm{H}), 1.70(\mathrm{~s}$, $3 \mathrm{H}), 1.39$ (s, 3H), 0.17 (s, 9H). ${ }^{13} \mathbf{C}$ NMR (50 MHz, $\left.\mathrm{CDCl}_{3}\right) \delta 135.4,129.7,110.8,100.2,94.2$, 
88.9, 73.8, 37.6, 27.4, 26.4 (2C), 24.5, 21.7, -0.5 (3C). IR $\left(\mathrm{CHCl}_{3}\right)$ v 3053, 2938, 2857, 1457, 1379, 1249, 1208, 1188, 1163, 1096, 1069, 1042, 980, 939, 893, 843, 760, 738, 704, 654, 559, 524, 407. MS (GCMS) : 356.1, 358.1 [M+·].

$(4 S *, 5 S *)-6-b r o m o-4-e t h y n y l-2,2-d i m e t h y l-1,3-d i o x a s p i r o[4.6]$ undec-6-ene (27)

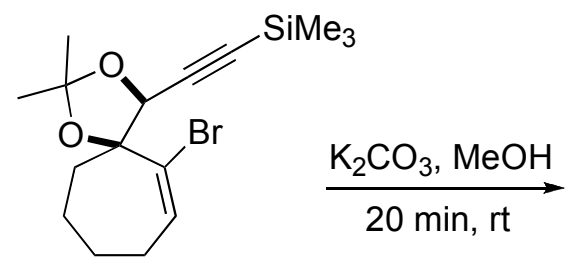

34

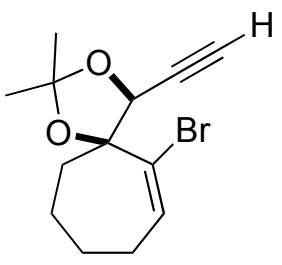

27

A solution of diol anti $34(2.89 \mathrm{~g}, 8.09 \mathrm{mmol}, 1 \mathrm{eq})$ and $\mathrm{K}_{2} \mathrm{CO}_{3}(1.12 \mathrm{~g}, 8.09 \mathrm{mmol}, 1 \mathrm{eq})$ in $\mathrm{MeOH}$ $(40 \mathrm{~mL})$ was stirred for $20 \mathrm{~min}$ at room temperature. The mixture was quenched by addition of a saturated solution of $\mathrm{NH}_{4} \mathrm{Cl}(40 \mathrm{~mL})$. After extraction with AcOEt, the combined organic layers were washed with water, brine, dried over $\mathrm{MgSO}_{4}$ and concentrated in vacuo. $2.25 \mathrm{~g}$ (97\%) of product were obtained as a white powder, and used without purification.

$\mathrm{C}_{13} \mathrm{H}_{17} \mathrm{BrO}_{2}, \mathrm{MW}=285.18 \mathrm{~g} \cdot \mathrm{mol}^{-1}$

$\mathbf{R f}=0,58\left(\right.$ Heptane $\left./ \mathrm{Et}_{2} \mathrm{O} 7: 3\right)$

$\mathbf{M p}=78^{\circ} \mathrm{C}$

${ }^{1} \mathbf{H}$ NMR $\left(200 \mathrm{MHz}, \mathrm{CDCl}_{3}\right) \delta 6.59(\mathrm{t}, J=6.6 \mathrm{~Hz}, 1 \mathrm{H}), 4.87(\mathrm{~d}, J=2.3 \mathrm{~Hz}, 1 \mathrm{H}), 2.65(\mathrm{~d}, J=2.3$ $\mathrm{Hz}, 1 \mathrm{H}), 2.30-1.54(\mathrm{~m}, 8 \mathrm{H}), 1.72(\mathrm{~s}, 3 \mathrm{H}), 1.41(\mathrm{~s}, 3 \mathrm{H}) .{ }^{13} \mathbf{C}$ NMR $\left(50 \mathrm{MHz}, \mathrm{CDCl}_{3}\right) \delta 136.3,129.2$, 111.0, 88.6, 78.7, 77.2, 73.4, 37.7, 27.4, 26.5, 26.4, 24.6, 21.7. IR $\left(\mathrm{CHCl}_{3}\right) \vee 3853,3751,3648$, 3283, 2938, 2360, 1684, 1653, 1558, 1541, 1507, 1457, 1375, 1261, 1209, 1163, 1068, 1040, 866, 669, 518, 419. MS (GCMS) : 284.1, $286.1[\mathrm{M}+\cdot]$.

\section{3-[(4S*,5S*)-6-bromo-2,2-dimethyl-1,3-dioxaspiro[4.6] undec-6-en-4-yl]prop-2-yn-1-ol (29)}

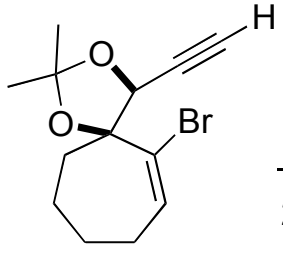

27 $\frac{\text { 1) } n \text {-BuLi, }-78^{\circ} \mathrm{C}, 30 \mathrm{~min}}{\text { 2) }\left(\mathrm{CH}_{2} \mathrm{O}\right)_{\mathrm{n}},-78^{\circ} \mathrm{C}, 1 \mathrm{~h}}$ $\mathrm{rt}, 18 \mathrm{~h}$

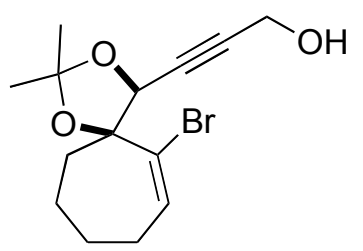

29

To a solution of compound $27(2.14 \mathrm{~g}, 7.50 \mathrm{mmol}, 1 \mathrm{eq})$ in THF $(63 \mathrm{~mL})$ was added dropwise $n$ $\operatorname{BuLi}\left(5.1 \mathrm{~mL}, 8.25 \mathrm{mmol}, 1.1 \mathrm{eq}, 1.6 \mathrm{M}\right.$ in hexane) at $-78^{\circ} \mathrm{C}$. The resulting solution was stirred for $30 \mathrm{~min}$ at $-78{ }^{\circ} \mathrm{C}$ and paraformaldehyde $(356 \mathrm{mg}, 11.25 \mathrm{mmol}, 1.5 \mathrm{eq})$ was then added. The reaction mixture was kept at $-78{ }^{\circ} \mathrm{C}$ for $1 \mathrm{~h}$ and then allowed to warm up to room temperature for $18 \mathrm{~h}$ before addition of a saturated solution of $\mathrm{NaHCO}_{3}(70 \mathrm{~mL})$. After extraction with $\mathrm{Et}_{2} \mathrm{O}$, the combined organic layers were washed with water, brine, dried over $\mathrm{MgSO}_{4}$ and concentrated in vacuo. The crude product was purified by chromatography on silica gel (elution with pentane/AcOEt 8:2) to afford $1.715 \mathrm{~g}(73 \%)$ of the product as a white solid.

$\mathrm{C}_{14} \mathrm{H}_{19} \mathrm{BrO}_{3}, \mathrm{MW}=315.2 \mathrm{~g} \cdot \mathrm{mol}^{-1}$

$\mathbf{R f}=0.24($ pentane/AcOEt 8:2) 
$\mathbf{M p}=72^{\circ} \mathrm{C}$

${ }^{1}$ H NMR $\left(200 \mathrm{MHz}, \mathrm{CDCl}_{3}\right) \delta 6.57(\mathrm{t}, J=6.8 \mathrm{~Hz}, 1 \mathrm{H}), 4.88(\mathrm{t}, J=2.1 \mathrm{~Hz}, 1 \mathrm{H}), 4.34(\mathrm{~d}, J=2.1$ $\mathrm{Hz}, 2 \mathrm{H}), 2.29-1.64(\mathrm{~m}, 9 \mathrm{H}), 1.71(\mathrm{~s}, 3 \mathrm{H}), 1.41(\mathrm{~s}, 3 \mathrm{H}) .{ }^{13} \mathbf{C} \mathbf{N M R}\left(50 \mathrm{MHz}, \mathrm{CDCl}_{3}\right) \delta 136.3,129.3$, 110.9, 88.7, 87.3, 80.6, 73.6, 51.1, 37.6, 27.4, 26.5 (2C), 24.7, 21.6. IR $\left(\mathrm{CHCl}_{3}\right)$ v 3414, 2986, 2937, 2864, 2183, 2144, 1457, 1380, 1275, 1261, 1209, 1162, 1043, 898, 867, 764, 750, 559, 430. MS (GCMS) : 314.1, $316.1[\mathrm{M}+\cdot]$.

(2Z)-2-[(3a $\left.S^{*}, 9 \mathrm{a} R *\right)-2,2-d i m e t h y l-6,7,8,9-t e t r a h y d r o c y c l o h e p t a[1,4]$ cyclobuta[1,2-d][1,3]dioxol -4(3aH)-ylidene]-4-(trimethylsilyl)but-3-yn-1-ol (31)

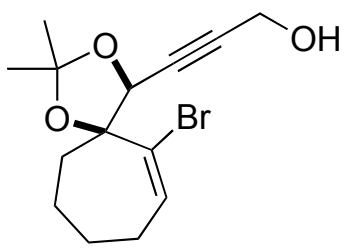

29

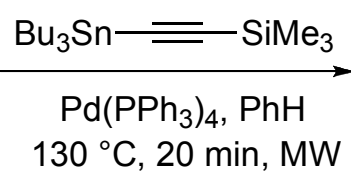

$130{ }^{\circ} \mathrm{C}, 20 \mathrm{~min}, \mathrm{MW}$

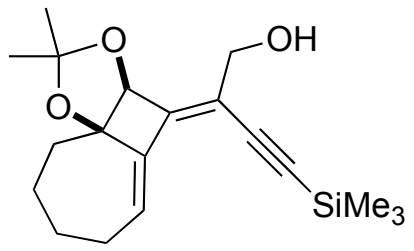

31

The reaction was carried out in a BIOTAGE sealed tube, under argon atmosphere. To a solution of the protected diol $29(1.182 \mathrm{~g}, 3.75 \mathrm{mmol}, 1 \mathrm{eq})$ in benzene $(15 \mathrm{~mL})$ were added $\mathrm{Pd}\left(\mathrm{PPh}_{3}\right)_{4}(433$ $\mathrm{mg}, 0.375 \mathrm{mmol}, 0.1 \mathrm{eq})$ and the stannylated reagent $(2.178 \mathrm{~g}, 5.63 \mathrm{mmol}, 1.5 \mathrm{eq})$. The yellow mixture was purged 10 min with argon and heated at $130{ }^{\circ} \mathrm{C}$ under microwave irradiation for 20 min. The resulted black solution was then cooled and concentrated. The reaction mixture was purified by chromatography on $5 \% \mathrm{Et}_{3} \mathrm{~N}$ treated silica gel (elution with pentane/AcOEt 92:8) to give $1.233 \mathrm{~g}(99 \%)$ of the product as a yellow solid.

$\mathrm{C}_{19} \mathrm{H}_{28} \mathrm{O}_{3} \mathrm{Si}, \mathrm{MW}=332.51$ g.mol ${ }^{-1}$

$\mathbf{R f}=0.23$ (pentane/AcOEt 92:8)

$\mathbf{M p}=36^{\circ} \mathrm{C}$

${ }^{1}$ H NMR $\left(300 \mathrm{MHz}, \mathrm{CDCl}_{3}\right) \delta 6.66(\mathrm{dd}, J=7.5 \mathrm{~Hz}, J=4.5 \mathrm{~Hz}, 1 \mathrm{H}), 4.84(\mathrm{~s}, 1 \mathrm{H}), 4.25(\mathrm{~m}, 2 \mathrm{H})$, $2.44(\mathrm{~m}, 1 \mathrm{H}), 2.25(\mathrm{~m}, 2 \mathrm{H}), 1.92(\mathrm{~m}, 4 \mathrm{H}), 1.75(\mathrm{~m}, 2 \mathrm{H}), 1.47(\mathrm{~s}, 3 \mathrm{H}), 1.42(\mathrm{~s}, 3 \mathrm{H}), 0.21(\mathrm{~s}, 9 \mathrm{H}) .{ }^{13} \mathbf{C}$ NMR $\left(75 \mathrm{MHz}, \mathrm{CDCl}_{3}\right) \delta 146.8,144.6,131.0,117.2,114.5,103.8,103.0,90.1,81.8,62.7,34.5$, 29.5, 28.8 (2C), 27.7, 26.7, -0.0 (3C). IR $\left(\mathrm{CHCl}_{3}\right) \vee$ 3438, 2926, 2855, 2132, 1443, 1370, 1250, 1230, 1208, 1192, 1148, 1106, 1054, 1010, 899, 922, 875, 841, 759, 698, 636, $555 \mathrm{~cm}^{-1}$. MS (GCMS) : 332.2 [M+·]. COSY, NOESY, HSQC, HMBC.

$(2 Z, 5 E)-2-\left[\left(3 \mathrm{a} S *, 9 \mathrm{a} R^{*}\right)-2,2-\right.$ dimethyl-6,7,8,9-tetrahydrocyclohepta[1,4]cyclobuta[1,2-d][1,3] dioxol-4(3aH)-ylidene]-7-methyloct-5-en-3-yne-1,7-diol (5a)

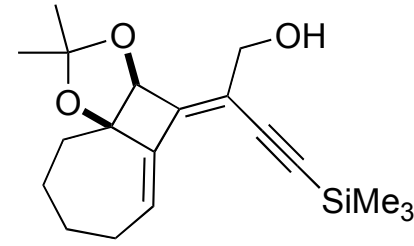

31

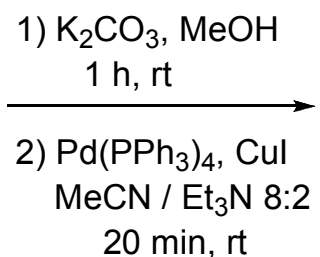

$>>^{\mathrm{OH}}$

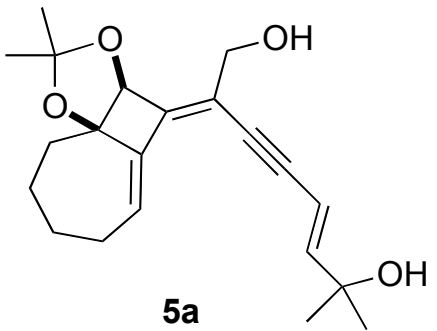

$5 a$

A solution of compound 31 (150 mg, $0.45 \mathrm{mmol}, 1 \mathrm{eq})$ and $\mathrm{K}_{2} \mathrm{CO}_{3}(62 \mathrm{mg}, 0.45 \mathrm{mmol}, 1 \mathrm{eq})$ in $\mathrm{MeOH}(2.25 \mathrm{~mL})$ was stirred at room temperature under argon for $1 \mathrm{~h}$. Then the mixture was 
quenched by addition of water $(3 \mathrm{~mL})$. After extraction with $\mathrm{Et}_{2} \mathrm{O}$, the combined organic layers were washed with water, brine, dried over $\mathrm{MgSO}_{4}$ and concentrated. It is important to note that this terminal alkyne 33 should not be dried, in order to avoid polymerization. Before the total evaporation of $\mathrm{Et}_{2} \mathrm{O}$ was added $\mathrm{MeCN}(4.5 \mathrm{~mL})$. When the residual $\mathrm{Et}_{2} \mathrm{O}$ was evapored, the product was then engaged in the Sonogashira reaction. To the mixture were added the vinyl iodide (115 $\mathrm{mg}$, $0.54 \mathrm{mmol}, 1.2 \mathrm{eq}), \mathrm{Pd}\left(\mathrm{PPH}_{3}\right)_{4}(26 \mathrm{mg}, 0.023 \mathrm{mmol}, 0.05 \mathrm{eq}), \mathrm{CuI}$ (13 mg, $\left.0.07 \mathrm{mmol}, 0.15 \mathrm{eq}\right)$ and $\mathrm{Et}_{3} \mathrm{~N}$ (1.1 mL, MeCN/Et $\left.{ }_{3} \mathrm{~N} 8: 2\right)$. According to the TLC, the reaction was completed after 20 min of stirring at room temperature under argon. The opaque yellow solution became a clear orange solution. Ethanolamine $(41 \mu \mathrm{L}, 0.68 \mathrm{mmol}, 10 \mathrm{eq} / \mathrm{CuI})$ was then added. The mixture was stirred for $1 \mathrm{~h}$ at room temperature, and became green, with the formation of precipitate. Then the mixture was quenched by addition of water $(5 \mathrm{~mL})$. After extraction with $\mathrm{Et}_{2} \mathrm{O}$, the combined organic layers were washed with water, brine, dried over $\mathrm{MgSO}_{4}$ and concentrated. The crude product was purified by chromatography on $5 \% \mathrm{Et}_{3} \mathrm{~N}$ treated silica gel (elution with pentane/AcOEt 7:3) to give $126 \mathrm{mg}(81 \%)$ of the product as an orange oil.

$\mathrm{C}_{21} \mathrm{H}_{28} \mathrm{O}_{4}, \mathrm{MW}=344.44$ g.mol ${ }^{-1}$

$\mathbf{R f}=0.14$ (pentane/AcOEt 7:3)

${ }^{1}$ H NMR $\left(300 \mathrm{MHz}, \mathrm{CDCl}_{3}\right) \delta 6.61(\mathrm{dd}, J=7.9 \mathrm{~Hz}, J=4.5 \mathrm{~Hz}, 1 \mathrm{H}), 6.29(\mathrm{~d}, J=16.0 \mathrm{~Hz}, 1 \mathrm{H})$, $5.95(\mathrm{~d}, J=16.0 \mathrm{~Hz}, 1 \mathrm{H}), 4.86(\mathrm{~s}, 1 \mathrm{H}), 4.32(\mathrm{~d}, J=13.6 \mathrm{~Hz}, 1 \mathrm{H}), 4.24(\mathrm{~d}, J=13.6 \mathrm{~Hz}, 1 \mathrm{H}), 2.44$ $(\mathrm{m}, 1 \mathrm{H}), 2.25(\mathrm{~m}, 2 \mathrm{H}), 1.98-1.35(\mathrm{~m}, 7 \mathrm{H}), 1.47(\mathrm{~s}, 3 \mathrm{H}), 1.43(\mathrm{~s}, 3 \mathrm{H}), 1.35(\mathrm{~s}, 6 \mathrm{H}) .{ }^{\mathbf{1 3}} \mathbf{C ~ N M R}(50$ $\left.\mathrm{MHz}_{1} \mathrm{CDCl}_{3}\right) \delta 151.2,145.5,144.6,130.7,117.4,114.5,106.9,96.5,90.0,88.1,81.7,71.0,62.9$, 34.5, 29.5 (2C), 28.8 (2C), 27.8, 26.8. IR $\left(\mathrm{CHCl}_{3}\right) \vee$ 3382, 2975, 2930, 2858, 1652, 1457, 1371, 1307, 1261, 1230, 1209, 1192, 1148, 1087, 1054, 1006, 957, 928, $844 \mathrm{~cm}^{-1}$. MS (GCMS) : 344.2 $[\mathrm{M}+\cdot]$.

\section{$(2 Z, 5 E)-2-\left[\left(3 a S^{*}, 9 \mathrm{a} R *\right)-2,2-d i m e t h y l-6,7,8,9-t e t r a h y d r o c y c l o h e p t a[1,4]\right.$ cyclobuta[1,2-d][1,3]} dioxol-4(3aH)-ylidene]dodec-5-en-3-yn-1-ol (5b)

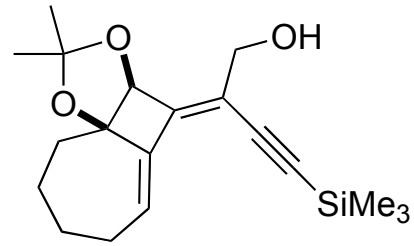

31

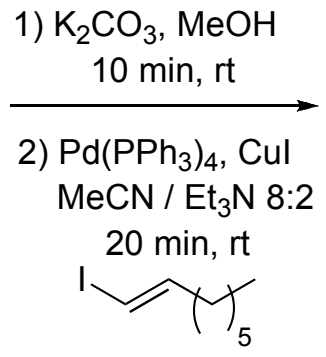

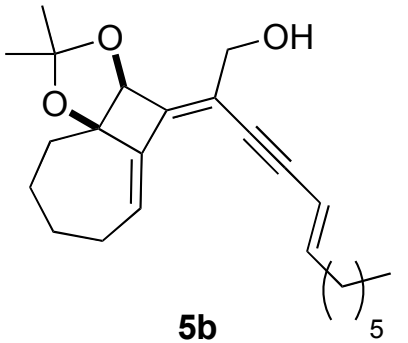

$5 b$

Compound $\mathbf{5 b}$ was prepared following the same procedure than for the preparation of $\mathbf{5 a}$, starting from compound 31 (180 mg, $0.54 \mathrm{mmol}, 1 \mathrm{eq})$ and $\mathrm{K}_{2} \mathrm{CO}_{3}(75 \mathrm{mg}, 0.54 \mathrm{mmol}, 1 \mathrm{eq})$ in $\mathrm{MeOH}$ (2.7 $\mathrm{mL}$ ). After $10 \mathrm{~min}$ of reaction and extraction, the alkyne 33 was reacted with the vinyl iodide (154 $\mathrm{mg}, 0.65 \mathrm{mmol}, 1.2 \mathrm{eq}), \mathrm{Pd}\left(\mathrm{PPh}_{3}\right)_{4}(31 \mathrm{mg}, 0.027 \mathrm{mmol}, 0.05 \mathrm{eq}), \mathrm{CuI}$ (15 mg, $\left.0.08 \mathrm{mmol}, 0.15 \mathrm{eq}\right)$, in $\mathrm{MeCN} / \mathrm{Et}_{3} \mathrm{~N} 8: 2(6.8 \mathrm{~mL})$. The mixture was stirred for $20 \mathrm{~min}$ at room temperature. Ethanolamine $(50 \mu \mathrm{L}, 0.81 \mathrm{mmol}, 10 \mathrm{eq} / \mathrm{CuI})$ was then added, and the solution was stirred for 1 hour. After extraction and purification by chromatography on $5 \% \mathrm{Et}_{3} \mathrm{~N}$ treated silica gel (elution with pentane/AcOEt 93:7), $168 \mathrm{mg}(84 \%)$ of the product were obtained as an orange oil.

$\mathrm{C}_{24} \mathrm{H}_{34} \mathrm{O}_{3}, \mathrm{MW}=370.52$ g.mol ${ }^{-1}$

$\mathbf{R f}=0.29$ (pentane/AcOEt 92:8)

${ }^{1}$ H NMR $\left(300 \mathrm{MHz}, \mathrm{CDCl}_{3}\right) \delta 6.61(\mathrm{dd}, J=7.6 \mathrm{~Hz}, J=4.9 \mathrm{~Hz}, 1 \mathrm{H}), 6.20(\mathrm{dt}, J=15.9 \mathrm{~Hz}, J=7.0$ $\mathrm{Hz}, 1 \mathrm{H}), 5.68(\mathrm{dt}, J=15.9 \mathrm{~Hz}, J=1.4 \mathrm{~Hz}, 1 \mathrm{H}), 4.86(\mathrm{~s}, 1 \mathrm{H}), 4.32(\mathrm{~d}, J=14.2 \mathrm{~Hz}, 1 \mathrm{H}), 4.23(\mathrm{~d}, J=$ 
$14.2 \mathrm{~Hz}, 1 \mathrm{H}), 2.53-1.56(\mathrm{~m}, 11 \mathrm{H}), 1.48(\mathrm{~s}, 3 \mathrm{H}), 1.43(\mathrm{~s}, 3 \mathrm{H}), 1.28(\mathrm{~m}, 8 \mathrm{H}), 0.88(\mathrm{t}, J=6.7 \mathrm{~Hz}, 3 \mathrm{H})$. ${ }^{13} \mathrm{C}$ NMR $\left(75 \mathrm{MHz}, \mathrm{CDCl}_{3}\right) \delta 146.2,145.3,144.7,130.6,117.6,114.6,109.6,97.6,90.2,86.1$, 81.8, 63.3, 34.7, 33.4, 31.8, 29.7, 28.9, 28.8 (2C), 27.9, 26.9, 22.7, 14.2. IR $\left(\mathrm{CHCl}_{3}\right) \vee$ 3433, 2925, 2855, 2360, 1651, 1612, 1456, 1379, 1370, 1306, 1259, 1230, 1209, 1192, 1150, 1087, 1054, 1007, $951,928,894,841,691,556 \mathrm{~cm}^{-1}$. MS (GCMS) : $370.3[\mathrm{M}+\cdot]$.

1-\{(1E,5Z)-5-[(3aS*,9a $\left.R^{*}\right)-2,2-$ dimethyl-6,7,8,9-tetrahydrocyclohepta[1,4]cyclobuta[1,2-d][1,3] dioxol-4(3aH)-ylidene]-6-hydroxyhex-1-en-3-ynyl\} cyclohexanol (5c)

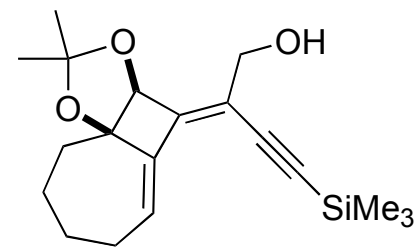

31
1) $\mathrm{K}_{2} \mathrm{CO}_{3}, \mathrm{MeOH}$ $20 \mathrm{~min}, \mathrm{rt}$

2) $\mathrm{Pd}\left(\mathrm{PPh}_{3}\right)_{4}, \mathrm{Cul}$ $\mathrm{MeCN} / \mathrm{Et}_{3} \mathrm{~N} 8: 2$ $20 \mathrm{~min}, \mathrm{rt}$<smiles>OC1(/C=C/I)CCCCC1</smiles>

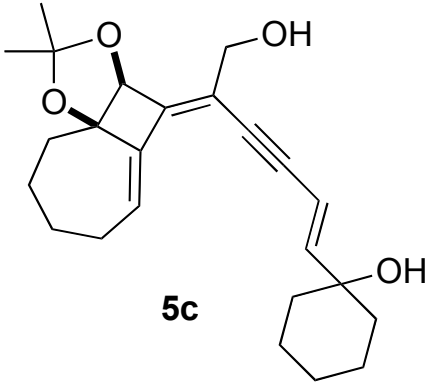

Compound $\mathbf{5 c}$ was prepared following the same procedure than for the preparation of $\mathbf{5 a}$, starting from compound 31 (220 mg, $0.66 \mathrm{mmol}, 1 \mathrm{eq})$ and $\mathrm{K}_{2} \mathrm{CO}_{3}(91 \mathrm{mg}, 0.66 \mathrm{mmol}, 1 \mathrm{eq})$ in $\mathrm{MeOH}$ (3.3 $\mathrm{mL}$ ). After $20 \mathrm{~min}$ of reaction and extraction, the alkyne $\mathbf{3 3}$ was reacted with the vinyl iodide (200 $\mathrm{mg}, 0.79 \mathrm{mmol}, 1.2 \mathrm{eq}), \mathrm{Pd}\left(\mathrm{PPh}_{3}\right)_{4}(38 \mathrm{mg}, 0.033 \mathrm{mmol}, 0.05 \mathrm{eq}), \mathrm{CuI}$ (19 mg, $\left.0.10 \mathrm{mmol}, 0.15 \mathrm{eq}\right)$, in $\mathrm{MeCN} / \mathrm{Et}_{3} \mathrm{~N} 8: 2(8.3 \mathrm{~mL})$. The mixture was stirred for $20 \mathrm{~min}$ at room temperature. Ethanolamine $(60 \mu \mathrm{L}, 0.99 \mathrm{mmol}, 10 \mathrm{eq} / \mathrm{CuI})$ was then added, and the solution was stirred for 1 hour. After extraction and purification by chromatography on $5 \% \mathrm{Et}_{3} \mathrm{~N}$ treated silica gel (elution with pentane/AcOEt 7:3), $216 \mathrm{mg}(85 \%)$ of the product were obtained as a yellow solid.

$\mathrm{C}_{24} \mathrm{H}_{32} \mathrm{O}_{4}, \mathrm{MW}=384.51$ g.mol ${ }^{-1}$

$\mathbf{R f}=0.26($ pentane/AcOEt 7:3)

$\mathbf{M p}=64^{\circ} \mathrm{C}$

${ }^{1}$ H NMR $\left(300 \mathrm{MHz}, \mathrm{CDCl}_{3}\right) \delta 6.62(\mathrm{dd}, J=7.8 \mathrm{~Hz}, J=4.7 \mathrm{~Hz}, 1 \mathrm{H}), 6.30(\mathrm{~d}, J=15.9 \mathrm{~Hz}, 1 \mathrm{H})$, $5.99(\mathrm{~d}, J=15.9 \mathrm{~Hz}, 1 \mathrm{H}), 4.86(\mathrm{~s}, 1 \mathrm{H}), 4.32(\mathrm{dd}, J=13.6 \mathrm{~Hz}, J=6.6 \mathrm{~Hz}, 1 \mathrm{H}), 4.24(\mathrm{dd}, J=13.6$ $\mathrm{Hz}, J=6.6 \mathrm{~Hz}, 1 \mathrm{H}), 2.45(\mathrm{~m}, 1 \mathrm{H}), 2.29(\mathrm{~m}, 1 \mathrm{H}), 2.17(\mathrm{t}, J=6.6 \mathrm{~Hz}, 1 \mathrm{H}: \mathrm{OH}), 2.04(\mathrm{~s}, 1 \mathrm{H}: \mathrm{OH})$, $1.93(\mathrm{~m}, 4 \mathrm{H}), 1.79-1.36(\mathrm{~m}, 12 \mathrm{H}), 1.47(\mathrm{~s}, 3 \mathrm{H}), 1.43(\mathrm{~s}, 3 \mathrm{H}) .{ }^{13} \mathbf{C} \mathbf{N M R}\left(75 \mathrm{MHz}, \mathrm{CDCl}_{3}\right) \delta 151.3$, $145.8,144.6,130.9,117.4,114.6,107.5,96.9,90.1,88.2,81.8,72.1,63.2,37.6$ (2C), 34.7, 29.7, 28.9 (2C), 27.9, 26.8, 25.4, 21.9 (2C). IR $\left(\mathrm{CHCl}_{3}\right) \vee$ 3396, 2986, 2930, 2856, 1448, 1380, 1370, $1261,1231,1209,1191,1150,1088,1054,1006,957,909,845,833,733,417,405 \mathrm{~cm}^{-1}$. MS (GCMS) : $384.3[\mathrm{M}+\cdot]$.

1-\{(1E,5Z)-5-[(3a $\left.S^{*}, 9 \mathrm{a} R^{*}\right)-2,2-$ dimethyl-6,7,8,9-tetrahydrocyclohepta[1,4]cyclobuta[1,2-d][1,3] dioxol-4(3aH)-ylidene]-6-hydroxyhex-1-en-3-ynyl\} cyclopentanol (5d) 


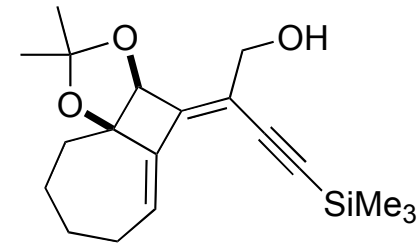

31

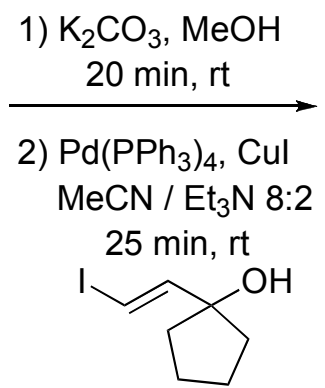

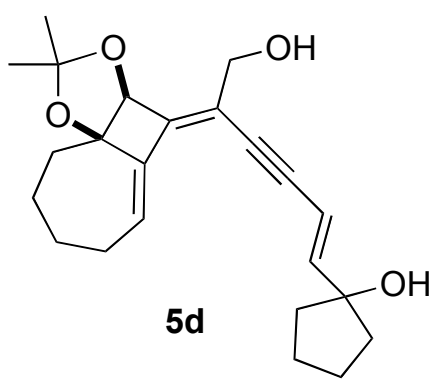

Compound 5d was prepared following the same procedure than for the preparation of 5a, starting from compound 31 (150 mg, $0.45 \mathrm{mmol}, 1 \mathrm{eq})$ and $\mathrm{K}_{2} \mathrm{CO}_{3}(62 \mathrm{mg}, 0.45 \mathrm{mmol}, 1 \mathrm{eq})$ in $\mathrm{MeOH}(2.3$ $\mathrm{mL}$ ). After $20 \mathrm{~min}$ of reaction and extraction, the alkyne $\mathbf{3 3}$ was reacted with the vinyl iodide (129 $\mathrm{mg}, 0.54 \mathrm{mmol}, 1.2 \mathrm{eq}), \mathrm{Pd}\left(\mathrm{PPh}_{3}\right)_{4}(26 \mathrm{mg}, 0.023 \mathrm{mmol}, 0.05 \mathrm{eq}), \mathrm{CuI}$ (13 mg, $0.068 \mathrm{mmol}, 0.15$ eq), in $\mathrm{MeCN} / \mathrm{Et}_{3} \mathrm{~N} 8: 2(5.6 \mathrm{~mL})$. The mixture was stirred for $25 \mathrm{~min}$ at room temperature. Ethanolamine $(40 \mu \mathrm{L}, 0.68 \mathrm{mmol}, 10 \mathrm{eq} / \mathrm{CuI})$ was then added, and the solution was stirred for 1 hour. After extraction and purification by chromatography on $5 \% \mathrm{Et}_{3} \mathrm{~N}$ treated silica gel (elution with pentane/AcOEt 75:25), $143 \mathrm{mg}(86 \%)$ of the product were obtained as an orange solid.

$\mathrm{C}_{23} \mathrm{H}_{30} \mathrm{O}_{4}, \mathrm{MW}=370.48$ g. $\mathrm{mol}^{-1}$

$\mathbf{R f}=0.18$ (pentane/AcOEt 75:25)

$\mathrm{Mp}=44^{\circ} \mathrm{C}$

${ }^{1}$ H NMR $\left(300 \mathrm{MHz}, \mathrm{CDCl}_{3}\right) \delta 6.62(\mathrm{dd}, J=7.8 \mathrm{~Hz}, J=4.7 \mathrm{~Hz}, 1 \mathrm{H}), 6.33(\mathrm{~d}, J=15.9 \mathrm{~Hz}, 1 \mathrm{H})$, $6.03(\mathrm{~d}, J=15.9 \mathrm{~Hz}, 1 \mathrm{H}), 4.87(\mathrm{~s}, 1 \mathrm{H}), 4.32(\mathrm{dd}, J=13.4 \mathrm{~Hz}, J=5.9 \mathrm{~Hz}, 1 \mathrm{H}), 4.24(\mathrm{dd}, J=13.4$ $\mathrm{Hz}, J=5.9 \mathrm{~Hz}, 1 \mathrm{H}), 2.50-2.17(\mathrm{~m}, 3 \mathrm{H}), 1.98-1.62(\mathrm{~m}, 15 \mathrm{H}), 1.48(\mathrm{~s}, 3 \mathrm{H}), 1.43(\mathrm{~s}, 3 \mathrm{H}) .{ }^{13} \mathbf{C} \mathbf{N M R}$ $\left(75 \mathrm{MHz}, \mathrm{CDCl}_{3}\right) \delta 150.1,145.5,144.6,130.7,117.4,114.5,107.1,96.8,90.0,88.1,82.2,81.7$, 63.0, 40.7 (2C), 34.6, 29.6, 28.9, 28.8, 27.8, 26.8, 23.9 (2C). IR $\left(\mathrm{CHCl}_{3}\right)$ v 3392, 2931, 2858, 2156, $1653,1436,1370,1261,1231,1210,1192,1149,1088,1054,1007,957,845,555,409 \mathrm{~cm}^{-1}$. MS (GCMS) : 370.2 [M+·].

\section{$(2 Z, 5 E)-2-\left[\left(3 \mathrm{a} S^{*}, 9 \mathrm{a} R^{*}\right)-2,2-\right.$ dimethyl-6,7,8,9-tetrahydrocyclohepta[1,4]cyclobuta[1,2-d][1,3] dioxol-4(3aH)-ylidene]-8-phenyloct-5-en-3-yn-1-ol (5e)}

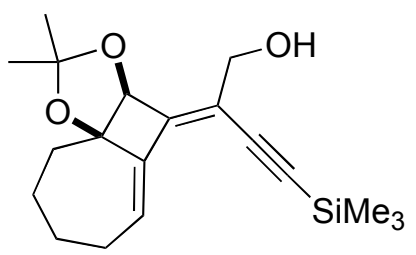

31
1) $\mathrm{K}_{2} \mathrm{CO}_{3}, \mathrm{MeOH}$ $20 \mathrm{~min}, \mathrm{rt}$

2) $\mathrm{Pd}\left(\mathrm{PPh}_{3}\right)_{4}, \mathrm{Cul}$ $\mathrm{MeCN} / \mathrm{Et}_{3} \mathrm{~N}$ 8:2 $30 \mathrm{~min}, \mathrm{rt}$<smiles>I/C=C/CCc1ccccc1</smiles>

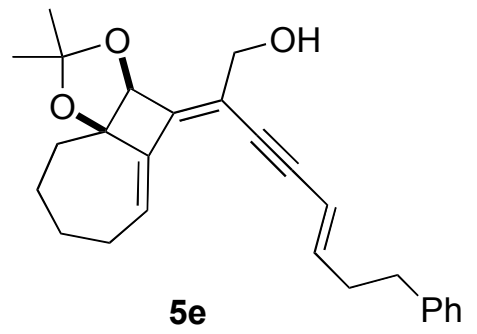

$5 e$

Compound 5e was prepared following the same procedure than for the preparation of 5a, starting from compound 31 (140 mg, $0.42 \mathrm{mmol}, 1 \mathrm{eq}$ ) and $\mathrm{K}_{2} \mathrm{CO}_{3}$ (58 mg, $\left.0.42 \mathrm{mmol}, 1 \mathrm{eq}\right)$ in $\mathrm{MeOH}(2.1$ $\mathrm{mL}$ ). After $20 \mathrm{~min}$ of reaction and extraction, the alkyne $\mathbf{3 3}$ was reacted with the vinyl iodide (163 $\mathrm{mg}, 0.63 \mathrm{mmol}, 1.5 \mathrm{eq}), \mathrm{Pd}\left(\mathrm{PPh}_{3}\right)_{4}(24 \mathrm{mg}, 0.02 \mathrm{mmol}, 0.05 \mathrm{eq}), \mathrm{CuI}$ (12 mg, $\left.0.06 \mathrm{mmol}, 0.15 \mathrm{eq}\right)$, in $\mathrm{MeCN} / \mathrm{Et}_{3} \mathrm{~N} 8: 2(5.3 \mathrm{~mL})$. The mixture was stirred for $30 \mathrm{~min}$ at room temperature. Ethanolamine $(0.04 \mathrm{~mL}, 0.60 \mathrm{mmol}, 10 \mathrm{eq} / \mathrm{CuI})$ was then added, and the solution was stirred for 1 hour. After extraction and purification by chromatography on $5 \% \mathrm{Et}_{3} \mathrm{~N}$ treated silica gel (elution with pentane/AcOEt 9:1), $130 \mathrm{mg}(79 \%)$ of the product were obtained as an orange oil.

$\mathrm{C}_{26} \mathrm{H}_{30} \mathrm{O}_{3}, \mathrm{MW}=390.51$ g.mol ${ }^{-1}$ 
$\mathbf{R f}=0.26($ pentane/AcOEt $85: 15)$

${ }^{1}$ H NMR $\left(300 \mathrm{MHz}, \mathrm{CDCl}_{3}\right) \delta 7.32-7.17(\mathrm{~m}, 5 \mathrm{H}), 6.59(\mathrm{dd}, J=7.9 \mathrm{~Hz}, J=4.5 \mathrm{~Hz}, 1 \mathrm{H}), 6.22(\mathrm{dt}, J$ $=15.8 \mathrm{~Hz}, J=7.2 \mathrm{~Hz}, 1 \mathrm{H}), 5.72(\mathrm{~d}, J=15.8 \mathrm{~Hz}, 1 \mathrm{H}), 4.86(\mathrm{~s}, 1 \mathrm{H}), 4.31(\mathrm{dd}, J=13.6 \mathrm{~Hz}, J=6.4$ $\mathrm{Hz}, 1 \mathrm{H}), 4.23(\mathrm{dd}, J=13.6 \mathrm{~Hz}, J=6.4 \mathrm{~Hz}, 1 \mathrm{H}), 2.74(\mathrm{t}, J=7.2 \mathrm{~Hz}, 2 \mathrm{H}), 2.48(\mathrm{~m}, 3 \mathrm{H}), 2.30(\mathrm{~m}$, $1 \mathrm{H}), 2.13(\mathrm{t}, J=6.4 \mathrm{~Hz}, 1 \mathrm{H}: \mathrm{OH}), 1.93(\mathrm{~m}, 4 \mathrm{H}), 1.80-1.58(\mathrm{~m}, 2 \mathrm{H}), 1.48(\mathrm{~s}, 3 \mathrm{H}), 1.43(\mathrm{~s}, 3 \mathrm{H})$. ${ }^{13}$ C NMR (75 MHz, $\left.\mathrm{CDCl}_{3}\right) \delta 145.5,144.63,144.57,141.2,130.6,128.53$ (2C), 128.50 (2C), 126.2, 117.5, 114.6, 110.4, 97.2, 90.1, 86.5, 81.8, 63.3, 35.2, 35.1, 34.7, 29.7, 28.94, 28.92, 27.9, 26.8. IR $\left(\mathrm{CHCl}_{3}\right) \vee$ 3448, 3026, 2987, 2929, 2855, 2331, 1605, 1497, 1454, 1379, 1370, 1306, 1260, 1231, 1209, 1192, 1149, 1087, 1054, 1007, 952, 842, 747, 699, $556 \mathrm{~cm}^{-1}$. MS (GCMS) : $390.2[\mathrm{M}+\cdot]$.

$(2 Z, 5 E)-2-\left[\left(3 a S^{*}, 9 \mathrm{a} R^{*}\right)-2,2-d i m e t h y l-6,7,8,9-\right.$ tetrahydrocyclohepta[1,4]cyclobuta $[1,2-d][1,3]$ dioxol-4(3aH)-ylidene]-7-isobutyl-9-methyldec-5-en-3-yne-1,7-diol (5f)

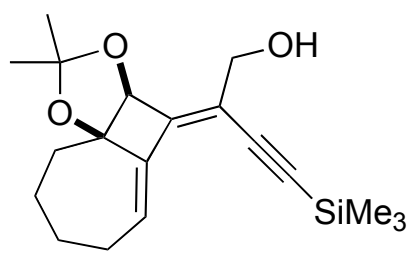

31
1) $\mathrm{K}_{2} \mathrm{CO}_{3}, \mathrm{MeOH}$ $20 \mathrm{~min}, \mathrm{rt}$

2) $\mathrm{Pd}\left(\mathrm{PPh}_{3}\right)_{4}, \mathrm{Cul}$ $\mathrm{MeCN} / \mathrm{Et}_{3} \mathrm{~N} 8: 2$ $20 \mathrm{~min}, \mathrm{rt}$

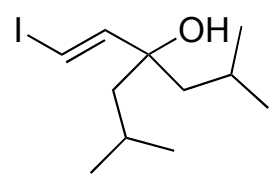

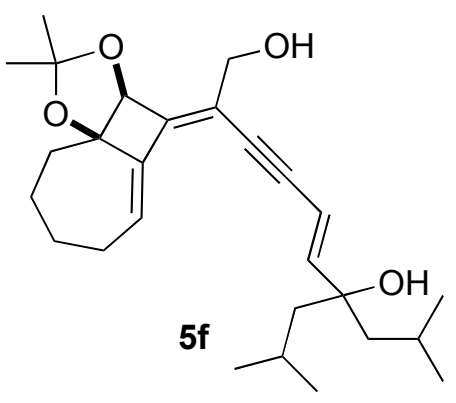

Compound $\mathbf{5 f}$ was prepared following the same procedure than for the preparation of 5a, starting from compound 31 (180 mg, $0.54 \mathrm{mmol}, 1 \mathrm{eq})$ and $\mathrm{K}_{2} \mathrm{CO}_{3}(75 \mathrm{mg}, 0.54 \mathrm{mmol}, 1 \mathrm{eq})$ in $\mathrm{MeOH}(2.7$ $\mathrm{mL}$ ). After $20 \mathrm{~min}$ of reaction and extraction, the alkyne 33 was reacted with the vinyl iodide (193 $\mathrm{mg}, 0.65 \mathrm{mmol}, 1.2 \mathrm{eq}), \mathrm{Pd}\left(\mathrm{PPh}_{3}\right)_{4}(31 \mathrm{mg}, 0.027 \mathrm{mmol}, 0.05 \mathrm{eq}), \mathrm{CuI}$ (15 mg, $\left.0.08 \mathrm{mmol}, 0.15 \mathrm{eq}\right)$, in $\mathrm{MeCN}_{2} \mathrm{Et}_{3} \mathrm{~N}$ 8:2 $(6.8 \mathrm{~mL})$. The mixture was stirred for $20 \mathrm{~min}$ at room temperature. Ethanolamine $(50 \mu \mathrm{L}, 0.81 \mathrm{mmol}, 10 \mathrm{eq} / \mathrm{CuI})$ was then added, and the solution was stirred for 1 hour. After extraction and purification by chromatography on $5 \% \mathrm{Et}_{3} \mathrm{~N}$ treated silica gel (elution with pentane/AcOEt 8:2), $187 \mathrm{mg}$ (81\%) of the product were obtained as an orange oil.

$\mathrm{C}_{27} \mathrm{H}_{40} \mathrm{O}_{4}, \mathrm{MW}=428.6$ g.mol ${ }^{-1}$

$\mathbf{R f}=0.22($ pentane/AcOEt $8: 2)$

${ }^{1}$ H NMR $\left(300 \mathrm{MHz}, \mathrm{CDCl}_{3}\right) \delta 6.61(\mathrm{dd}, J=7.5 \mathrm{~Hz}, J=4.4 \mathrm{~Hz}, 1 \mathrm{H}), 6.16(\mathrm{~d}, J=15.9 \mathrm{~Hz}, 1 \mathrm{H})$, $5.97(\mathrm{~d}, J=15.9 \mathrm{~Hz}, 1 \mathrm{H}), 4.87(\mathrm{~s}, 1 \mathrm{H}), 4.33(\mathrm{dd}, J=13.4 \mathrm{~Hz}, J=6.1 \mathrm{~Hz}, 1 \mathrm{H}), 4.25(\mathrm{dd}, J=13.4$ $\mathrm{Hz}, J=6.1 \mathrm{~Hz}, 1 \mathrm{H}), 2.43(\mathrm{~m}, 1 \mathrm{H}), 2.23(\mathrm{~m}, 2 \mathrm{H}), 1.97(\mathrm{~m}, 4 \mathrm{H}), 1.74(\mathrm{~m}, 3 \mathrm{H}), 1.59(\mathrm{~s}, 1 \mathrm{H}: \mathrm{OH})$, 1.49 (s, 3H), $1.46(\mathrm{~d}, J=5.9 \mathrm{~Hz}, 4 \mathrm{H}), 1.43(\mathrm{~s}, 3 \mathrm{H}), 1.34(\mathrm{~s}, 1 \mathrm{H}: \mathrm{OH}), 0.94(\mathrm{~d}, J=6.7 \mathrm{~Hz}, 6 \mathrm{H}), 0.91$ $(\mathrm{d}, J=6.7 \mathrm{~Hz}, 6 \mathrm{H}) .{ }^{13} \mathrm{C}$ NMR $\left(75 \mathrm{MHz}, \mathrm{CDCl}_{3}\right) \delta 150.3,145.3,144.7,130.5,117.6,114.5,107.6$, 97.1, 90.0, 87.8, 81.7, 76.9, 62.9, 51.0 (2C), 34.6, 29.5, 28.8 (2C), 27.8, 26.8, 24.7 (2C), 24.6 (2C), 24.0 (2C). IR $\left(\mathrm{CHCl}_{3}\right) \vee 3431,2951,2927,2868,1651,1467,1380,1370,1304,1260,1231,1210$, $1192,1150,1087,1054,1008,963,894,845,555 \mathrm{~cm}^{-1}$. MS (GCMS) : 428.3 [M+·]. 


\section{- Synthesis of cyclooctatrienes 16a and 18a-f}

2-[(3a $R *, 4 S *, 5 R *, 9 \mathrm{~b} S *)-9-($ hydroxymethyl)-2,2-dimethyl-4,9b-dihydro-5H-3a,4-propanocyclo octa[3,4]cyclobuta[1,2- $d][1,3]$ dioxol-5-yl]propan-2-ol

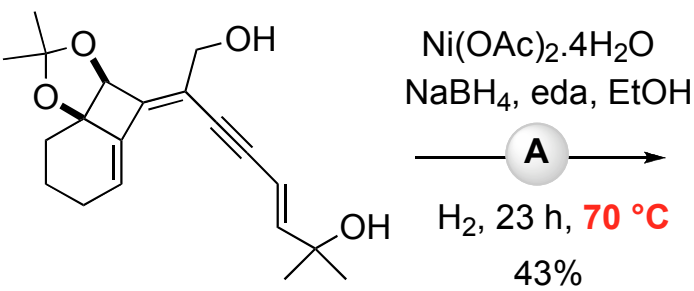

$4 a$

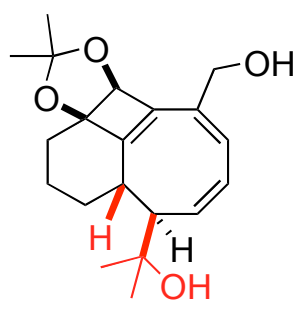

$16 a$
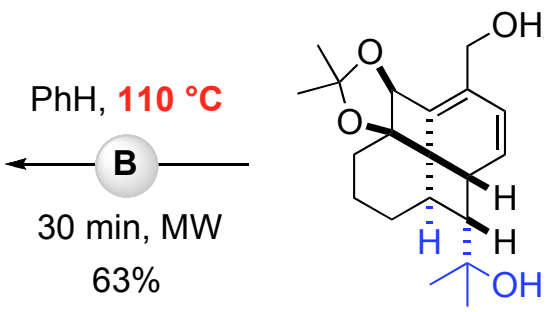

21a

\section{- Path A : semihydrogenation of trienyne 4 a at $70^{\circ} \mathrm{C}$}

To a solution of $\mathrm{Ni}(\mathrm{OAc})_{2} .4 \mathrm{H}_{2} \mathrm{O}(68 \mathrm{mg}, 0.27 \mathrm{mmol}, 1 \mathrm{eq})$ in $\mathrm{EtOH}(0.68 \mathrm{~mL})$ at room temperature under argon was added a solution of $\mathrm{NaBH}_{4}(10 \mathrm{mg}, 0.27 \mathrm{mmol}, 1 \mathrm{eq})$ in EtOH $(0.22 \mathrm{~mL})$. After stirring $1 \mathrm{~h}$ under one atmosphere of $\mathrm{H}_{2}$, a solution of trienyne $4 \mathbf{a}(90 \mathrm{mg}, 0.27 \mathrm{mmol}, 1 \mathrm{eq})$ and ethylenediamine $(0.06 \mathrm{~mL}, 0.95 \mathrm{mmol}, 3.5 \mathrm{eq})$ in $\mathrm{EtOH}(0.56 \mathrm{~mL})$ was added. The black mixture was stirred at $70{ }^{\circ} \mathrm{C}$ for $23 \mathrm{~h}$ and after a complete conversion, was filtrated on $5 \% \mathrm{Et}_{3} \mathrm{~N}$ treated silica gel with AcOEt, and concentrated. The crude product was purified by chromatography on 5\% $\mathrm{Et}_{3} \mathrm{~N}$ treated silica gel (elution with pentane/AcOEt 5:5) to give $39 \mathrm{mg}$ (43\%) of the product as a white solid.

\section{- $\quad$ Path B : heating of fenestradiene 21a}

The reaction was carried out in a BIOTAGE sealed tube, under argon atmosphere. A solution of the fenestradiene 21 a $(70 \mathrm{mg}, 0.21 \mathrm{mmol}, 1 \mathrm{eq})$ in benzene $(5 \mathrm{~mL})$ was purged $5 \mathrm{~min}$ with argon and heated at $110{ }^{\circ} \mathrm{C}$ under microwave irradiation for $30 \mathrm{~min}$. The resulted solution was then cooled and concentrated. According to the ${ }^{1} \mathrm{H}$ NMR, the conversion of fenestradiene in cyclooctatriene was complete. The reaction mixture was then purified by chromatography on $5 \% \mathrm{Et}_{3} \mathrm{~N}$ treated silica gel (elution with pentane/AcOEt 5:5) to give $44 \mathrm{mg}$ (63\%) of the product as a white solid.

$\mathrm{C}_{20} \mathrm{H}_{28} \mathrm{O}_{4}, \mathrm{MW}=332.42$ g. $\mathrm{mol}^{-1}$

$\mathbf{R f}=0.37$ (heptane/Et ${ }_{2} \mathrm{O} 1: 9$ )

$\mathbf{M p}=180^{\circ} \mathrm{C}$

${ }^{1} \mathbf{H}$ NMR $\left(200 \mathrm{MHz}, \mathrm{CDCl}_{3}\right) \delta 6.07(\mathrm{~d}, J=4.4 \mathrm{~Hz}, 1 \mathrm{H}), 5.98(\mathrm{dd}, J=11.0 \mathrm{~Hz}, J=4.6 \mathrm{~Hz}, 1 \mathrm{H})$, $5.64(\mathrm{dd}, J=11.0 \mathrm{~Hz}, J=9.0 \mathrm{~Hz}, 1 \mathrm{H}), 4.93(\mathrm{~s}, 1 \mathrm{H}), 4.27$ (br s, 2H), $2.64(\mathrm{~m}, 2 \mathrm{H}), 2.17-1.61$ (m, $8 \mathrm{H}), 1.36(\mathrm{~s}, 3 \mathrm{H}), 1.33(\mathrm{~s}, 3 \mathrm{H}), 1.27(\mathrm{~s}, 3 \mathrm{H}), 1.16(\mathrm{~s}, 3 \mathrm{H}) .{ }^{13} \mathbf{C}$ NMR $\left(50 \mathrm{MHz}, \mathrm{CDCl}_{3}\right) \delta 157.2$ $\left(\mathrm{C}_{\text {quat }}\right), 140.7(+), 137.2\left(\mathrm{C}_{\text {quat }}\right), 134.6\left(\mathrm{C}_{\text {quat }}\right), 127.2(+), 127.1(+), 114.7\left(\mathrm{C}_{\text {quat }}\right), 85.4\left(\mathrm{C}_{\text {quat }}\right), 83.9$ $(+), 72.8\left(\mathrm{C}_{\text {quat }}\right), 64.9(-), 51.8(+), 41.2(+), 34.1(-), 33.3(-), 30.8(+), 29.7(+), 28.7(+), 24.2(+)$, $23.8(-)$. IR $\left(\mathrm{CHCl}_{3}\right) \vee 3676,3649,3630,3399,2927,2854,2031,2024,2008,1995,1970,1918$, 1458, 1369, 1261, 1234, 1206, 1184, 1143, 1075, 1057, 1017, 929, 840, 775, 711, 572. HRMS (ESI, positive ion): calc'd for $\left(\mathrm{C}_{20} \mathrm{H}_{28} \mathrm{O}_{4} \mathrm{Li}\right)^{+} 339.2143$; found 339.2137 . X-Ray analysis : see Table 1

Single crystals of compound 16a were obtained at room temperature in a crystallisation tube (height $=15 \mathrm{~cm}$, diameter $=0.4 \mathrm{~cm})$, upon slow diffusion of $n$-hexane $(0.19 \mathrm{~mL})$ into a chloroform solution $(0.06 \mathrm{~mL})$ containing compound $16 \mathbf{a}(5 \mathrm{mg})$.

CCDC 707562 for $\mathbf{1 6 a}$ contains the supplementary crystallographic data for this paper. These data can be obtained free of charge via www.ccdc.cam.ac.uk/conts/retrieving.html (from the Cambridge 
Crystallographic Data Center, 12, Union Road, Cambridge CB21EZ, UK ; fax : (+44) 1223-336033 ; or deposit@ccdc.cam.ac.uk).

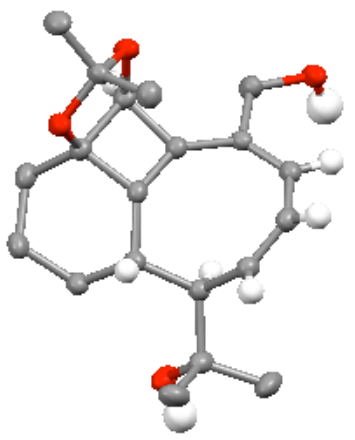

The majority of $\mathrm{H}$ are omitted for clarity. The crystal data were collected at $173 \mathrm{~K}$ for complex on a Kappa CCD diffractometer, using monochromated Mo K $\alpha$ radiation $(\lambda=0.71073 \AA)$. Details of data collection parameters and refinements results are listed in the following Table. The structures were solved using direct methods. After refinement of the non hydrogen atoms, difference-Fourier maps revealed maxima of residual electron density close to positions expected for hydrogen atoms. Hydrogen atoms were introduced as fixed contributors at calculated positions $(\mathrm{C}-\mathrm{H}=0.95 \AA, \mathrm{B}(\mathrm{H})$ $=1.3 \mathrm{Beqv}$ ). Final difference maps revealed no significant maxima. All calculations were done using the Nonius OpenMoleN package (OpenMoleN, Interactive Structure Solutions, Nonius B.V., Delft, The Netherlands, 1997). Neutral atom scattering factor coefficients and anormalous dispersion coefficients were taken from a standard source (D. T. Cromer, J. T. Waber, International Tables for X-Ray Crystallography, 1974, Vol IV, The Kynoch Press, Birmingham).

Table 1 : X-Ray experimental data

\begin{tabular}{|c|c|}
\hline Formula & $\mathrm{C}_{20} \mathrm{H}_{28} \mathrm{O}_{4}$ \\
\hline Molecular weight $M_{r}$ & 332.42 \\
\hline Crystal system & Monoclinic \\
\hline Space group & $\mathrm{P} 21 / \mathrm{c}$ \\
\hline $\mathrm{a}(\AA)$ & $8.1351(2)$ \\
\hline $\mathrm{b}(\AA)$ & $21.0319(10)$ \\
\hline $\mathrm{c}(\AA)$ & $10.6795(5)$ \\
\hline$\alpha(\mathrm{deg})$ & 90.00 \\
\hline$\beta(\operatorname{deg})$ & $98.594(2)$ \\
\hline$\gamma(\mathrm{deg})$ & 90.00 \\
\hline $\mathrm{V}\left(\AA^{3}\right)$ & $1806.71(13)$ \\
\hline $\mathrm{Z}$ & 4 \\
\hline Color & Colorless \\
\hline Crystal dim (mm) & $0.45 * 0.40 * 0.35$ \\
\hline$\rho_{\text {calcd }}\left(\right.$ g.cm $\left.{ }^{-3}\right)$ & 1.222 \\
\hline F000 & 720 \\
\hline$\mu\left(\mathrm{mm}^{-1}\right)$ & 0.084 \\
\hline Temperature $(\mathrm{K})$ & $173(2)$ \\
\hline Wavelength $(\AA)$ & 0.71073 \\
\hline Radiation & MoK $\alpha$ graphite monochromated \\
\hline Diffractometer & KappaCCD \\
\hline Scan mode & phi and omega scans \\
\hline Hkl limits & $-10,10 /-27,25 /-13,9$ \\
\hline Theta limits (deg) & $1.94 / 27.48$ \\
\hline Number of data meas. & 13039 \\
\hline
\end{tabular}




\begin{tabular}{|l|l|}
\hline $\begin{array}{l}\text { Number of data with } \\
\mathrm{I}>2 \sigma(\mathrm{I})\end{array}$ & 4132 \\
\hline R & 0.0523 \\
\hline Rw & 0.1314 \\
\hline
\end{tabular}

\section{2-[(3a $\left.R^{*}, 4 S^{*}, 5 R *, 9 \mathrm{~b} S^{*}\right)-9-($ hydroxymethyl)-2,2-dimethyl-4,9b-dihydro-5H-3a,4-butano cycloocta[3,4] cyclobuta[1,2-d][1,3]dioxol-5-yl]propan-2-ol (18a)}

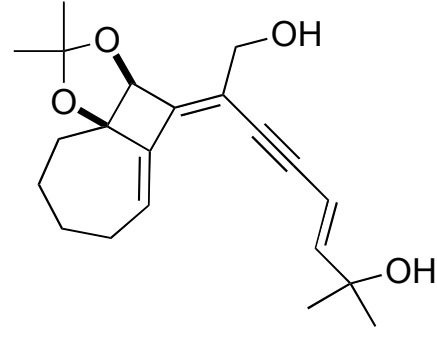

$5 a$

\author{
$\mathrm{Ni}(\mathrm{OAc})_{2} \cdot 4 \mathrm{H}_{2} \mathrm{O}$ \\ $\mathrm{NaBH}_{4}, \mathrm{EtOH}$ \\ ethylenediamine \\ $16 \mathrm{~h}, \mathrm{H}_{2}$, rt
}

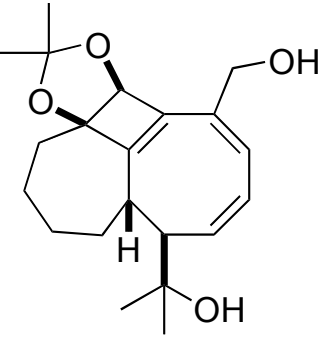

$18 \mathrm{a}$

To a solution of $\mathrm{Ni}(\mathrm{OAc})_{2} .4 \mathrm{H}_{2} \mathrm{O}(72 \mathrm{mg}, 0.29 \mathrm{mmol}, 1 \mathrm{eq})$ in $\mathrm{EtOH}(0.73 \mathrm{~mL})$ at room temperature under argon was added a solution of $\mathrm{NaBH}_{4}(11 \mathrm{mg}, 0.29 \mathrm{mmol}, 1 \mathrm{eq})$ in EtOH $(0.23 \mathrm{~mL})$. After stirring $1 \mathrm{~h}$ under one atmosphere of $\mathrm{H}_{2}$, a solution of trienyne $\mathbf{5 a}(100 \mathrm{mg}, 0.29 \mathrm{mmol}, 1 \mathrm{eq})$ and ethylenediamine $(69 \mu \mathrm{L}, 1.02 \mathrm{mmol}, 3.5 \mathrm{eq})$ in $\mathrm{EtOH}(0.60 \mathrm{~mL})$ was added. The black mixture was stirred overnight and after a complete conversion, was filtrated on 5\% $\mathrm{Et}_{3} \mathrm{~N}$ treated silica gel with AcOEt, and concentrated. The crude product was purified by chromatography on $5 \% \mathrm{Et}_{3} \mathrm{~N}$ treated silica gel (elution with pentane/AcOEt 6:4) to give $77 \mathrm{mg}(77 \%)$ of the product as a yellow solid.

$\mathrm{C}_{21} \mathrm{H}_{30} \mathrm{O}_{4}, \mathrm{M}=346.46 \mathrm{~g} \cdot \mathrm{mol}^{-1}$

$\mathbf{R f}=0.16$ (pentane/AcOEt 6:4)

$\mathbf{M p}=118^{\circ} \mathrm{C}$

${ }^{1} \mathbf{H}$ NMR $\left(300 \mathrm{MHz}, \mathrm{CDCl}_{3}\right) \delta 6.10(\mathrm{~d}, J=4.0 \mathrm{~Hz}, 1 \mathrm{H}), 5.97(\mathrm{dd}, J=10.7 \mathrm{~Hz}, J=4.0 \mathrm{~Hz}, 1 \mathrm{H})$, $5.64(\mathrm{t}, J=10.7 \mathrm{~Hz}, 1 \mathrm{H}), 4.83(\mathrm{~s}, 1 \mathrm{H}), 4.31(\mathrm{~d}, J=12.8 \mathrm{~Hz}, 1 \mathrm{H}), 4.24(\mathrm{~d}, J=12.8 \mathrm{~Hz}, 1 \mathrm{H}), 2.64(\mathrm{t}$, $J=10.7 \mathrm{~Hz}, 1 \mathrm{H}), 2.51(\mathrm{~m}, 2 \mathrm{H}), 2.32(\mathrm{~m}, 1 \mathrm{H}), 2.10(\mathrm{~m}, 2 \mathrm{H}), 1.97(\mathrm{~m}, 2 \mathrm{H}), 1.77(\mathrm{~m}, 3 \mathrm{H}), 1.57(\mathrm{~m}$, $1 \mathrm{H}), 1.35(\mathrm{~s}, 3 \mathrm{H}), 1.27(\mathrm{~s}, 3 \mathrm{H}), 1.22(\mathrm{~s}, 3 \mathrm{H}), 1.11(\mathrm{~s}, 3 \mathrm{H}) .{ }^{13} \mathbf{C}$ NMR $\left(75 \mathrm{MHz}, \mathrm{CDCl}_{3}\right) \delta 158.0$ $\left(\mathrm{C}_{\text {quat }}\right), 141.1\left(\mathrm{C}_{\text {quat }}\right), 139.5(+), 137.0\left(\mathrm{C}_{\text {quat }}\right), 127.5(+), 127.1(+), 114.7\left(\mathrm{C}_{\text {quat }}\right), 91.3\left(\mathrm{C}_{\text {quat }}\right), 82.0$ $(+), 73.5\left(\mathrm{C}_{\text {quat }}\right), 64.9(-), 52.2(+), 41.0(+), 36.9(-), 35.2(-), 32.0(+), 30.2(+), 29.7(-), 28.7(+)$, $24.7(-), 23.7(+)$. IR $\left(\mathrm{CHCl}_{3}\right)$ v 3649, 3503, 3412, 2975, 2924, 2852, 1647, 1559, 1541, 1507, 1458, 1449, 1396, 1368, 1314, 1278, 1244, 1207, 1139, 1112, 1042, 1025, 920, 861, 838, 561, 554, 548, 541, 407, $401 \mathrm{~cm}^{-1}$. HRMS (ESI, positive ion): calc'd for $\left(\mathrm{C}_{21} \mathrm{H}_{30} \mathrm{O}_{4} \mathrm{Li}\right)^{+} 353.2299$; found 353.2292. COSY, NOESY, HSQC, HMBC. X-Ray analysis : see Table 2

Single crystals of compound 18a were obtained at room temperature in a crystallisation tube (height $=15 \mathrm{~cm}$, diameter $=0.4 \mathrm{~cm})$, upon slow diffusion and slow evaporation of $n$-heptane $(0.5 \mathrm{~mL})$ into a benzene solution $(0.17 \mathrm{~mL})$ containing compound $18 \mathbf{a}(8 \mathrm{mg})$.

CCDC 653647 for $\mathbf{1 8 a}$ contains the supplementary crystallographic data for this paper. These data can be obtained free of charge via www.ccdc.cam.ac.uk/conts/retrieving.html (from the Cambridge Crystallographic Data Center, 12, Union Road, Cambridge CB21EZ, UK ; fax : (+44) 1223-336033 ; or deposit@.ccdc.cam.ac.uk). 


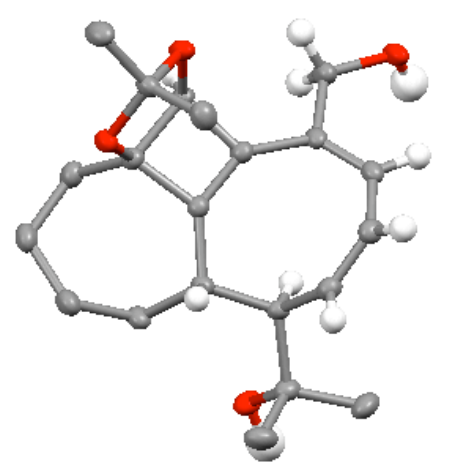

The majority of $\mathrm{H}$ are omitted for clarity. The crystal data were collected at $173 \mathrm{~K}$ for complex on a Kappa CCD diffractometer, using monochromated Mo K $\alpha$ radiation $(\lambda=0.71073 \AA)$. Details of data collection parameters and refinements results are listed in the following Table. The structures were solved using direct methods. After refinement of the non hydrogen atoms, difference-Fourier maps revealed maxima of residual electron density close to positions expected for hydrogen atoms. Hydrogen atoms were introduced as fixed contributors at calculated positions $(\mathrm{C}-\mathrm{H}=0.95 \AA, \mathrm{B}(\mathrm{H})$ $=1.3 \mathrm{Beqv}$ ). Final difference maps revealed no significant maxima. All calculations were done using the Nonius OpenMoleN package (OpenMoleN, Interactive Structure Solutions, Nonius B.V., Delft, The Netherlands, 1997). Neutral atom scattering factor coefficients and anormalous dispersion coefficients were taken from a standard source (D. T. Cromer, J. T. Waber, International Tables for X-Ray Crystallography, 1974, Vol IV, The Kynoch Press, Birmingham).

\section{Table 2 : X-Ray experimental data}

\begin{tabular}{|c|c|}
\hline Formula & $\mathrm{C}_{21} \mathrm{H}_{30} \mathrm{O}_{4}$ \\
\hline Molecular weight $M_{r}$ & 346.45 \\
\hline Crystal system & Monoclinic \\
\hline Space group & $\mathrm{P} 21 / \mathrm{c}$ \\
\hline $\mathrm{a}(\AA)$ & $8.2890(3)$ \\
\hline $\mathrm{b}(\AA)$ & $21.1559(7)$ \\
\hline $\mathrm{c}(\AA)$ & $10.7607(4)$ \\
\hline$\alpha(\operatorname{deg})$ & 90.00 \\
\hline$\beta(\mathrm{deg})$ & $100.360(2)$ \\
\hline$\gamma(\mathrm{deg})$ & 90.00 \\
\hline $\mathrm{V}\left(\AA^{3}\right)$ & $1856.25(11)$ \\
\hline Z & 4 \\
\hline Color & colorless \\
\hline Crystal dim (mm) & $0.40 * 0.20 * 0.15$ \\
\hline$\rho_{\text {calcd }}\left(\right.$ g.cm $\left.{ }^{-3}\right)$ & 1.240 \\
\hline F000 & 752 \\
\hline$\mu\left(\mathrm{mm}^{-1}\right)$ & 0.084 \\
\hline Temperature $(\mathrm{K})$ & 173 \\
\hline Wavelength $(\AA)$ & 0.71073 \\
\hline Radiation & MoK $\alpha$ graphite monochromated \\
\hline Diffractometer & KappaCCD \\
\hline Scan mode & phi and omega scans \\
\hline Hkl limits & $-10,10 /-27,24 /-13,13$ \\
\hline Theta limits (deg) & $1.93 / 27.48$ \\
\hline Number of data meas. & 4242 \\
\hline Number of data with $\mathrm{I}>2 \sigma(\mathrm{I})$ & 3164 \\
\hline $\mathrm{R}$ & 0.0444 \\
\hline
\end{tabular}




\begin{tabular}{|l|l|}
\hline Rw & 0.0995 \\
\hline
\end{tabular}

[ $\left(3 \mathrm{a} R *, 4 S *, 5 R *, 9 \mathrm{~b} S^{*}\right)-5$-hexyl-2,2-dimethyl-4,9b-dihydro-5H-3a,4-butanocycloocta[3,4] cyclobuta[1,2-d][1,3]dioxol-9-yl]methanol (18b)

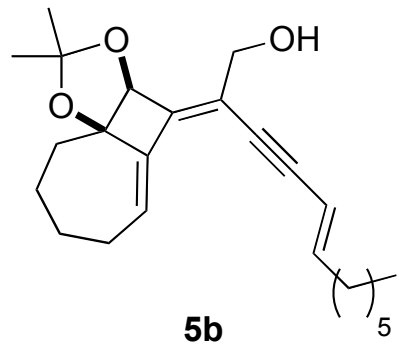

$$
\begin{gathered}
\underset{\mathrm{Ni}(\mathrm{OAc})_{2} \cdot 4 \mathrm{H}_{2} \mathrm{O}}{\mathrm{NaBH}_{4}, \mathrm{EtOH}} \\
\text { ethylenediamine } \\
33 \mathrm{~h}, \mathrm{H}_{2}, \mathrm{rt}
\end{gathered}
$$

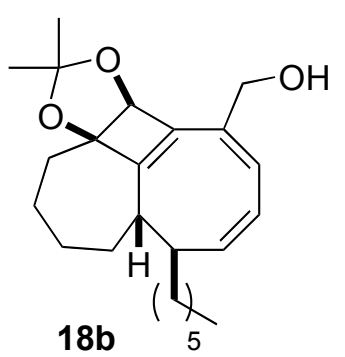

To a solution of $\mathrm{Ni}(\mathrm{OAc})_{2} .4 \mathrm{H}_{2} \mathrm{O}(80 \mathrm{mg}, 0.32 \mathrm{mmol}, 1 \mathrm{eq})$ in $\mathrm{EtOH}(0.80 \mathrm{~mL})$ at room temperature under argon was added a solution of $\mathrm{NaBH}_{4}(12 \mathrm{mg}, 0.32 \mathrm{mmol}, 1 \mathrm{eq})$ in $\mathrm{EtOH}(0.26 \mathrm{~mL})$. After stirring $1 \mathrm{~h}$ under one atmosphere of $\mathrm{H}_{2}$, a solution of trienyne $\mathbf{5 b}(120 \mathrm{mg}, 0.32 \mathrm{mmol}, 1 \mathrm{eq})$ and ethylenediamine $(0.08 \mathrm{~mL}, 1.12 \mathrm{mmol}, 3.5 \mathrm{eq})$ in EtOH $(0.67 \mathrm{~mL})$ was added. The black mixture was stirred overnight and after a complete conversion, was filtrated on $5 \% \mathrm{Et}_{3} \mathrm{~N}$ treated silica gel with AcOEt, and concentrated. The crude product was purified by chromatography on $5 \% \mathrm{Et}_{3} \mathrm{~N}$ treated silica gel (elution with pentane/ $\left.\mathrm{Et}_{2} \mathrm{O} 8: 2\right)$ to give $75 \mathrm{mg}(63 \%)$ of the product as a colorless oil.

$\mathrm{C}_{24} \mathrm{H}_{36} \mathrm{O}_{3}, \mathrm{M}=372.54 \mathrm{~g} \cdot \mathrm{mol}^{-1}$

$\mathbf{R f}=0.15$ (pentane/ $\left./ \mathrm{Et}_{2} \mathrm{O} 8: 2\right)$

${ }^{1} \mathbf{H}$ NMR $\left(300 \mathrm{MHz}, \mathrm{CDCl}_{3}\right) \delta 6.06(\mathrm{~d}, J=4.3 \mathrm{~Hz}, 1 \mathrm{H}), 5.94(\mathrm{dd}, J=11.1 \mathrm{~Hz}, J=4.3 \mathrm{~Hz}, 1 \mathrm{H})$, $5.70(\mathrm{dd}, J=11.1 \mathrm{~Hz}, J=9.4 \mathrm{~Hz}, 1 \mathrm{H}), 4.83(\mathrm{~s}, 1 \mathrm{H}), 4.26(\mathrm{~s}, 2 \mathrm{H}), 2.38-1.93(\mathrm{~m}, 7 \mathrm{H}), 1.76(\mathrm{~m}, 2 \mathrm{H})$, 1.51-1.06 (m, 12H), $1.35(\mathrm{~s}, 3 \mathrm{H}), 1.31(\mathrm{~s}, 3 \mathrm{H}), 0.84(\mathrm{t}, J=6.8 \mathrm{~Hz}, 3 \mathrm{H}) .{ }^{13} \mathbf{C} \mathbf{N M R}\left(75 \mathrm{MHz}, \mathrm{CDCl}_{3}\right)$ $\delta 157.7\left(\mathrm{C}_{\text {quat }}\right), 142.8(+), 141.3\left(\mathrm{C}_{\text {quat }}\right), 136.0\left(\mathrm{C}_{\text {quat }}\right), 127.5(+), 127.4(+), 114.4\left(\mathrm{C}_{\text {quat }}\right), 91.0$ $\left(\mathrm{C}_{\text {quat }}\right), 82.2(+), 65.3(-), 42.4(+), 42.1(+), 36.7(-), 35.3(-), 33.1(-), 31.9(-), 30.2(+), 29.8(-)$, $29.4(-), 28.7(+), 26.9(-), 24.9(-), 22.7(-), 14.2(+)$. IR $\left(\mathrm{CHCl}_{3}\right) \vee$ 3448, 2924, 2853, 2160, 1448, 1378, 1368, 1316, 1278, 1244, 1210, 1174, 1141, 1114, 1055, 1029, 861, 832, 774, 748, $707 \mathrm{~cm}^{-1}$. HRMS (ESI, positive ion): calc'd for $\left(\mathrm{C}_{24} \mathrm{H}_{36} \mathrm{O}_{3} \mathrm{Li}\right)^{+} 379.2820$; found 379.2811. COSY, NOESY, HSQC, HMBC.

\section{$1-[(3 \mathrm{a} R *, 4 S *, 5 R *, 9 \mathrm{~b} S *)-9-($ hydroxymethyl)-2,2-dimethyl-4,9b-dihydro-5H-3a,4-butano cycloocta[3,4] cyclobuta[1,2-d][1,3] dioxol-5-yl]cyclohexanol (18c)}

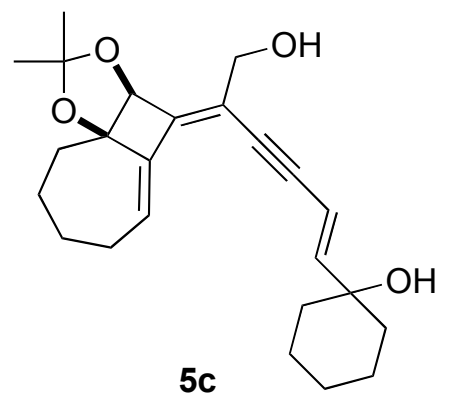

$$
\begin{gathered}
\underset{\mathrm{Ni}(\mathrm{OAc})_{2} \cdot 4 \mathrm{H}_{2} \mathrm{O}}{\mathrm{NaBH}}{ }_{4}, \mathrm{EtOH} \\
\text { ethylenediamine } \\
15 \mathrm{~h}, \mathrm{H}_{2}, \mathrm{rt}
\end{gathered}
$$

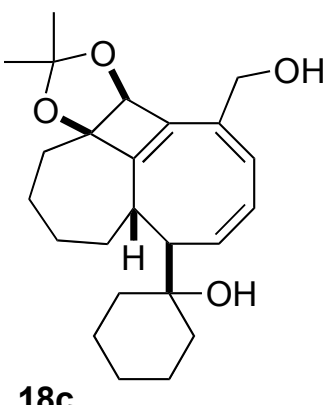

To a solution of $\mathrm{Ni}(\mathrm{OAc})_{2} .4 \mathrm{H}_{2} \mathrm{O}(113 \mathrm{mg}, 0.46 \mathrm{mmol}, 1 \mathrm{eq})$ in $\mathrm{EtOH}(1.14 \mathrm{~mL})$ at room temperature under argon was added a solution of $\mathrm{NaBH}_{4}(17 \mathrm{mg}, 0.46 \mathrm{mmol}, 1 \mathrm{eq})$ in $\mathrm{EtOH}(0.37$ 
$\mathrm{mL})$. After stirring $1 \mathrm{~h}$ under one atmosphere of $\mathrm{H}_{2}$, a solution of trienyne $\mathbf{5 c}(175 \mathrm{mg}, 0.46 \mathrm{mmol}, 1$ eq) and ethylenediamine $(0.11 \mathrm{~mL}, 1.59 \mathrm{mmol}, 3.5 \mathrm{eq})$ in EtOH $(0.95 \mathrm{~mL})$ was added. The black mixture was stirred overnight and after a complete conversion, was filtrated on $5 \% \mathrm{Et}_{3} \mathrm{~N}$ treated silica gel with AcOEt, and concentrated. The crude product was purified by chromatography on 5\% $\mathrm{Et}_{3} \mathrm{~N}$ treated silica gel (elution with pentane/AcOEt 75:25) to give $142 \mathrm{mg}(81 \%)$ of the product as a white solid.

$\mathrm{C}_{24} \mathrm{H}_{34} \mathrm{O}_{4}, \mathrm{M}=386.52 \mathrm{~g} \cdot \mathrm{mol}^{-1}$

$\mathbf{R f}=0.24($ pentane/AcOEt $7: 3$ )

$\mathbf{M p}=87^{\circ} \mathrm{C}$

${ }^{1}$ H NMR $\left(300 \mathrm{MHz}, \mathrm{CDCl}_{3}\right) \delta 6.10(\mathrm{~d}, J=4.0 \mathrm{~Hz}, 1 \mathrm{H}), 5.98(\mathrm{dd}, J=11.3 \mathrm{~Hz}, J=4.0 \mathrm{~Hz}, 1 \mathrm{H})$, $5.75(\mathrm{~m}, 1 \mathrm{H}), 4.83(\mathrm{~s}, 1 \mathrm{H}), 4.28(\mathrm{~s}, 2 \mathrm{H}), 2.57(\mathrm{~m}, 3 \mathrm{H}), 2.10(\mathrm{~m}, 1 \mathrm{H}), 1.97-1.07(\mathrm{~m}, 18 \mathrm{H}), 1.36(\mathrm{~s}$, 3H), $1.29(\mathrm{~s}, 3 \mathrm{H}) .{ }^{13} \mathrm{C}$ NMR $\left(75 \mathrm{MHz}, \mathrm{CDCl}_{3}\right) \delta 158.0\left(\mathrm{C}_{\text {quat }}\right), 140.9\left(\mathrm{C}_{\text {quat }}\right), 139.3(+), 136.8$ $\left(\mathrm{C}_{\text {quat }}\right), 127.3(+), 126.9(+), 114.7\left(\mathrm{C}_{\text {quat }}\right), 91.2\left(\mathrm{C}_{\text {quat }}\right), 82.0(+), 73.9\left(\mathrm{C}_{\text {quat }}\right), 64.8(-), 52.4(+), 40.3$ $(+), 38.4(2 \mathrm{C},(-)), 37.2(-), 35.1(-), 30.6(-), 30.2(+), 29.8(-), 28.7(+), 25.8(-), 24.7(-), 21.5(-)$. IR $\left(\mathrm{CHCl}_{3}\right) \vee$ 3648, 3416, 2926, 2853, 2248, 1725, 1448, 1378, 1369, 1317, 1277, 1244, 1206, 1138, 1112, 1030, 959, 922, 864, 838, 773, 734, 710, $419 \mathrm{~cm}^{-1}$. HRMS (ESI, positive ion): calc'd for $\left(\mathrm{C}_{24} \mathrm{H}_{34} \mathrm{O}_{4} \mathrm{Li}\right)^{+} 393.2612$; found 393.2602. COSY, NOESY, HSQC, HMBC.

\section{$1-[(3 \mathrm{a} R *, 4 S *, 5 R *, 9 \mathrm{~b} S *)-9-($ hydroxymethyl)-2,2-dimethyl-4,9b-dihydro-5H-3a,4-butano cycloocta $[3,4]$ cyclobuta[1,2-d][1,3]dioxol-5-yl]cyclopentanol (18d)}

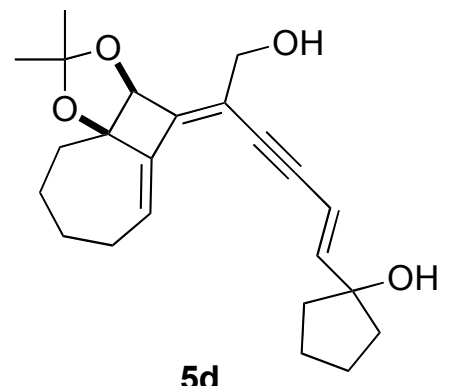

5d

$$
\begin{gathered}
\underset{\mathrm{Ni}(\mathrm{OAc})_{2} \cdot 4 \mathrm{H}_{2} \mathrm{O}}{\mathrm{NaBH}}, \mathrm{EtOH} \\
\text { ethylenediamine } \\
15 \mathrm{~h}, \mathrm{H}_{2}, \mathrm{rt}
\end{gathered}
$$
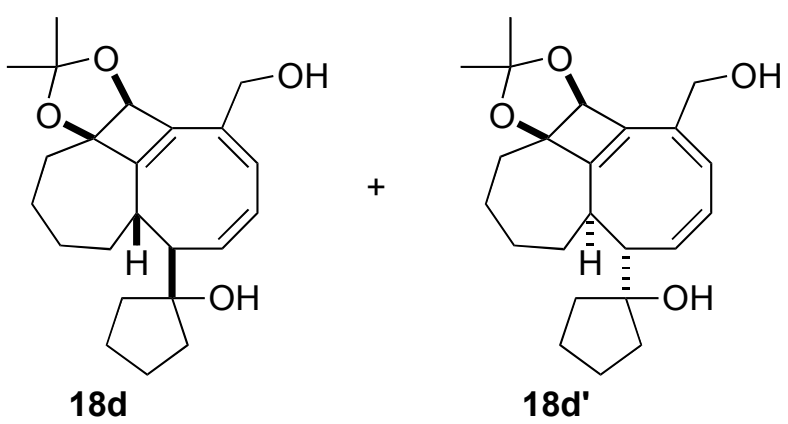

To a solution of $\mathrm{Ni}(\mathrm{OAc})_{2} .4 \mathrm{H}_{2} \mathrm{O}(96 \mathrm{mg}, 0.39 \mathrm{mmol}, 1 \mathrm{eq})$ in $\mathrm{EtOH}(0.98 \mathrm{~mL})$ at room temperature under argon was added a solution of $\mathrm{NaBH}_{4}(15 \mathrm{mg}, 0.39 \mathrm{mmol}, 1 \mathrm{eq})$ in EtOH $(0.31 \mathrm{~mL})$. After stirring $1 \mathrm{~h}$ under one atmosphere of $\mathrm{H}_{2}$, a solution of trienyne $\mathbf{5 d}$ (143 $\left.\mathrm{mg}, 0.39 \mathrm{mmol}, 1 \mathrm{eq}\right)$ and ethylenediamine $(0.09 \mathrm{~mL}, 1.37 \mathrm{mmol}, 3.5 \mathrm{eq})$ in $\mathrm{EtOH}(0.81 \mathrm{~mL})$ was added. The black mixture was stirred overnight and after a complete conversion, was filtrated on $5 \% \mathrm{Et}_{3} \mathrm{~N}$ treated silica gel with AcOEt, and concentrated. The crude product was purified by chromatography on $5 \% \mathrm{Et}_{3} \mathrm{~N}$ treated silica gel (elution with pentane/AcOEt 8:2) to give $78 \mathrm{mg}$ of a compound 18d' (54\%) as a white solid, and $39 \mathrm{mg}(27 \%)$ of the desired compound $\mathbf{1 8 d}$ as a white solid. The compound $\mathbf{1 8 d}$ ' rapidly rearranged at room temperature to give the desired compound $\mathbf{1 8 d}$. When the compound 18d' was heated at $80^{\circ} \mathrm{C}$ in benzene for $5 \mathrm{~min}$ under microwave irradiation, this product was totally rearranged in the desired compound $\mathbf{1 8 d}$ (final yield : 81\%).

Compound 18d' : (contamined with traces of compound 18d)

$\mathrm{C}_{23} \mathrm{H}_{32} \mathrm{O}_{4}, \mathrm{M}=372.50 \mathrm{~g} \cdot \mathrm{mol}^{-1}$

$\mathbf{R f}=0.56($ pentane/AcOEt 7:3)

$\mathbf{M p}=48^{\circ} \mathrm{C}$

${ }^{1}$ H NMR $\left(300 \mathrm{MHz}, \mathrm{CDCl}_{3}\right) \delta 6.02(\mathrm{~d}, J=3.1 \mathrm{~Hz}, 1 \mathrm{H}), 5.83(\mathrm{~m}, 2 \mathrm{H}), 4.91(\mathrm{~d}, J=2.2 \mathrm{~Hz}, 1 \mathrm{H}), 4.5$ $(\mathrm{d}, J=11.4 \mathrm{~Hz}, 1 \mathrm{H}), 4.02(\mathrm{t}, J=11.4 \mathrm{~Hz}, 1 \mathrm{H}), 3.49(\mathrm{~m}, 1 \mathrm{H}: \mathrm{OH}), 2.57(\mathrm{~m}, 1 \mathrm{H}), 2.36-1.37$ (m, $18 \mathrm{H}), 1.54(\mathrm{~s}, 3 \mathrm{H}), 1.42(\mathrm{~s}, 3 \mathrm{H}) .{ }^{13} \mathrm{C}$ NMR $\left(75 \mathrm{MHz}, \mathrm{CDCl}_{3}\right) \delta 159.5\left(\mathrm{C}_{\text {quat }}\right), 141.3(+), 138.2$ 
$\left(\mathrm{C}_{\text {quat }}\right), 133.2\left(\mathrm{C}_{\text {quat }}\right), 127.6(+), 123.0(+), 114.7\left(\mathrm{C}_{\text {quat }}\right), 89.8\left(\mathrm{C}_{\text {quat }}\right), 83.4\left(\mathrm{C}_{\text {quat }}\right), 82.4(+), 68.0(-)$, $55.1(+), 50.8(+), 42.7(-), 41.0(-), 34.8(-), 32.0(-), 30.7(-), 30.3(+), 29.1(+), 25.3(-), 25.0(-)$, $23.8(-) . \mathbf{I R}\left(\mathrm{CHCl}_{3}\right) \vee 3691,3675,3614,3587,2987,2361,2340,2014,1982,1967,1949,1560$, 1507, 1457, 1394, 1242, 1066, $669 \mathrm{~cm}^{-1}$. HRMS (ESI, positive ion): calc'd for $\left(\mathrm{C}_{23} \mathrm{H}_{32} \mathrm{O}_{4} \mathrm{Li}\right)^{+}$ 379.2456 ; found 379.2451. COSY, NOESY, HSQC, HMBC.

Compound 18d :

$\mathrm{C}_{23} \mathrm{H}_{32} \mathrm{O}_{4}, \mathrm{M}=372.50 \mathrm{~g} \cdot \mathrm{mol}^{-1}$

$\mathbf{R f}=0.20($ pentane/AcOEt 7:3)

$\mathbf{M p}=152^{\circ} \mathrm{C}$

${ }^{1}$ H NMR $\left(300 \mathrm{MHz}, \mathrm{CDCl}_{3}\right) \delta 6.11(\mathrm{~d}, J=4.0 \mathrm{~Hz}, 1 \mathrm{H}), 5.97(\mathrm{dd}, J=11.1 \mathrm{~Hz}, J=4.0 \mathrm{~Hz}, 1 \mathrm{H})$, $5.86(\mathrm{~m}, 1 \mathrm{H}), 4.84(\mathrm{~s}, 1 \mathrm{H}), 4.30(\mathrm{~s}, 2 \mathrm{H}), 2.64(\mathrm{~m}, 2 \mathrm{H}), 2.34(\mathrm{~m}, 1 \mathrm{H}), 2.17-1.09(\mathrm{~m}, 17 \mathrm{H}), 1.37(\mathrm{~s}$, $3 \mathrm{H}), 1.30(\mathrm{~s}, 3 \mathrm{H}) .{ }^{13} \mathbf{C}$ NMR $\left(75 \mathrm{MHz}, \mathrm{CDCl}_{3}\right) \delta 158.3\left(\mathrm{C}_{\text {quat }}\right), 140.9\left(\mathrm{C}_{\text {quat }}\right), 139.9(+), 136.4$ $\left(\mathrm{C}_{\text {quat }}\right), 127.2(+), 126.6(+), 114.7\left(\mathrm{C}_{\text {quat }}\right), 91.0\left(\mathrm{C}_{\text {quat }}\right), 84.3\left(\mathrm{C}_{\text {quat }}\right), 82.2(+), 65.0(-), 51.2(+), 41.7$ $(-), 41.4(+), 39.4(-), 36.3(-), 34.9(-), 30.2(+), 29.4(-), 28.7(+), 24.6(-), 24.5(-), 23.1(-)$. IR $\left(\mathrm{CHCl}_{3}\right) \vee 3417,2930,2852,2365,2357,2334,2210,2164,2148,1998,1447,1379,1369,1318$, 1275, 1243, 1206, 1140, 1113, 1025, 921, 841, 774, 733, 547, 418, $409 \mathrm{~cm}^{-1}$. HRMS (ESI, positive ion): calc'd for $\left(\mathrm{C}_{23} \mathrm{H}_{32} \mathrm{O}_{4} \mathrm{Li}\right)^{+} 379.2456$; found 379.2452. COSY, NOESY, HSQC, HMBC.

\section{[(3a $\left.R^{*}, 4 S^{*}, 5 R *, 9 \mathrm{~b} S^{*}\right)-2,2-d i m e t h y l-5-(2-p h e n y l e t h y l)-4,9 \mathrm{~b}-d i h y d r o-5 H-3 \mathrm{a}, 4-\mathrm{butano}$ cycloocta $[3,4]$ cyclobuta[1,2-d][1,3]dioxol-9-yl]methanol (18e)}

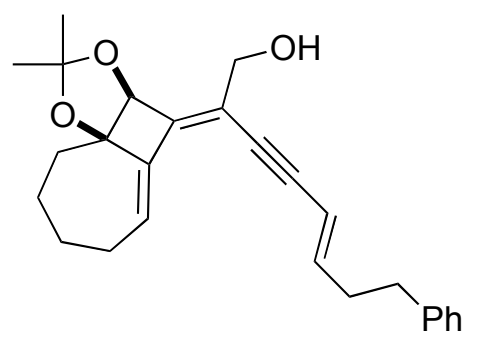

$5 e$

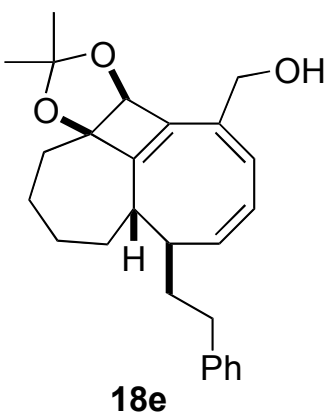

$18 \mathrm{e}$

To a solution of $\mathrm{Ni}(\mathrm{OAc})_{2} .4 \mathrm{H}_{2} \mathrm{O}(64 \mathrm{mg}, 0.26 \mathrm{mmol}, 1 \mathrm{eq})$ in $\mathrm{EtOH}(0.65 \mathrm{~mL})$ at room temperature under argon was added a solution of $\mathrm{NaBH}_{4}(10 \mathrm{mg}, 0.26 \mathrm{mmol}, 1 \mathrm{eq})$ in EtOH $(0.21 \mathrm{~mL})$. After stirring $1 \mathrm{~h}$ under one atmosphere of $\mathrm{H}_{2}$, a solution of trienyne 5e $(100 \mathrm{mg}, 0.26 \mathrm{mmol}, 1 \mathrm{eq})$ and ethylenediamine $(0.06 \mathrm{~mL}, 0.91 \mathrm{mmol}, 3.5 \mathrm{eq})$ in $\mathrm{EtOH}(0.54 \mathrm{~mL})$ was added. The black mixture was stirred overnight and after a complete conversion, was filtrated on $5 \% \mathrm{Et}_{3} \mathrm{~N}$ treated silica gel with AcOEt, and concentrated. The crude product was purified by chromatography on $5 \% \mathrm{Et}_{3} \mathrm{~N}$ treated silica gel (elution with pentane/AcOEt 9:1) to give $76 \mathrm{mg}(76 \%)$ of the product as a white oil.

$\mathrm{C}_{26} \mathrm{H}_{32} \mathrm{O}_{3}, \mathrm{M}=392.53 \mathrm{~g} \cdot \mathrm{mol}^{-1}$

$\mathbf{R f}=0.17$ (pentane/AcOEt 9:1)

${ }^{1}$ H NMR $\left(300 \mathrm{MHz}, \mathrm{CDCl}_{3}\right) \delta$ 7.29-7.12 (m, 5H), $6.11(\mathrm{~d}, J=4.4 \mathrm{~Hz}, 1 \mathrm{H}), 6.05(\mathrm{dd}, J=11.1 \mathrm{~Hz}, J$ $=4.4 \mathrm{~Hz}, 1 \mathrm{H}), 5.81(\mathrm{dd}, J=11.1 \mathrm{~Hz}, J=9.4 \mathrm{~Hz}, 1 \mathrm{H}), 4.84(\mathrm{~s}, 1 \mathrm{H}), 4.28(\mathrm{~d}, J=5.0 \mathrm{~Hz}, 2 \mathrm{H}), 2.68$ $(\mathrm{m}, 1 \mathrm{H}), 2.40(\mathrm{~m}, 2 \mathrm{H}), 2.27-1.49(\mathrm{~m}, 12 \mathrm{H}), 1.38(\mathrm{~s}, 3 \mathrm{H}), 1.34(\mathrm{~s}, 3 \mathrm{H}) .{ }^{13} \mathbf{C ~ N M R}\left(75 \mathrm{MHz}, \mathrm{CDCl}_{3}\right)$ $\delta 157.6\left(\mathrm{C}_{\text {quat }}\right), 142.5\left(\mathrm{C}_{\text {quat }}\right), 142.3(+), 141.4\left(\mathrm{C}_{\text {quat }}\right), 136.2\left(\mathrm{C}_{\text {quat }}\right), 128.5(2 \mathrm{C},(+)), 128.4(2 \mathrm{C},(+))$, $128.1(+), 127.4(+), 125.8(+), 114.5\left(\mathrm{C}_{\text {quat }}\right), 90.9\left(\mathrm{C}_{\text {quat }}\right), 82.2(+), 65.3(-), 42.4(+), 41.9(+), 36.6$ $(-), 35.3(-), 35.1(-), 33.4(-), 30.2(+), 29.7(-), 28.8(+), 24.9(-)$. IR $\left(\mathrm{CHCl}_{3}\right)$ v 3460, 3025, 2924, 2852, 2365, 1727, 1603, 1496, 1454, 1378, 1368, 1316, 1278, 1244, 1210, 1174, 1140, 1112, 1077 , 1032, 922, 861, 833, 775, 747, 699, 628, $511 \mathrm{~cm}^{-1}$. HRMS (ESI, positive ion): calc'd for 


\section{4-[(3a $R *, 4 S *, 5 R *, 9 \mathrm{~b} S *)-9-($ hydroxymethyl)-2,2-dimethyl-4,9b-dihydro-5H-3a,4-butano cycloocta[3,4]cyclobuta[1,2-d][1,3]dioxol-5-yl]-2,6-dimethylheptan-4-ol (18f)}

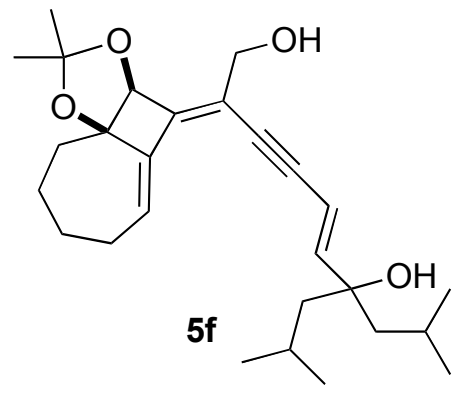

$$
\begin{gathered}
\underset{\mathrm{Ni}(\mathrm{OAc})_{2} \cdot 4 \mathrm{H}_{2} \mathrm{O}}{\stackrel{\mathrm{NaBH}}{4}, \mathrm{EtOH}} \\
\text { ethylenediamine } \\
22 \mathrm{~h}, \mathrm{H}_{2}, \mathrm{rt}
\end{gathered}
$$

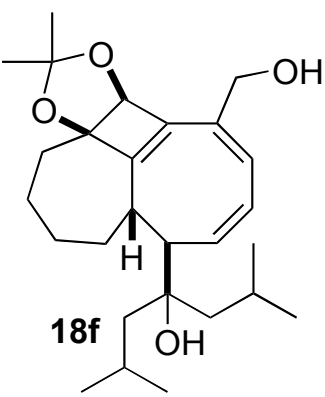

To a solution of $\mathrm{Ni}(\mathrm{OAc})_{2} .4 \mathrm{H}_{2} \mathrm{O}(70 \mathrm{mg}, 0.28 \mathrm{mmol}, 1 \mathrm{eq})$ in $\mathrm{EtOH}(0.70 \mathrm{~mL})$ at room temperature under argon was added a solution of $\mathrm{NaBH}_{4}(11 \mathrm{mg}, 0.28 \mathrm{mmol}, 1 \mathrm{eq})$ in EtOH $(0.23 \mathrm{~mL})$. After stirring $1 \mathrm{~h}$ under one atmosphere of $\mathrm{H}_{2}$, a solution of trienyne $\mathbf{5 f}(120 \mathrm{mg}, 0.28 \mathrm{mmol}, 1 \mathrm{eq})$ and ethylenediamine $(0.07 \mathrm{~mL}, 0.98 \mathrm{mmol}, 3.5 \mathrm{eq})$ in $\mathrm{EtOH}(0.58 \mathrm{~mL})$ was added. The black mixture was stirred overnight and after a complete conversion, was filtrated on $5 \% \mathrm{Et}_{3} \mathrm{~N}$ treated silica gel with AcOEt, and concentrated. The crude product was purified by chromatography on $5 \% \mathrm{Et}_{3} \mathrm{~N}$ treated silica gel (elution with pentane/AcOEt 8:2) to give $105 \mathrm{mg}(88 \%)$ of the product as a white solid.

$\mathrm{C}_{27} \mathrm{H}_{42} \mathrm{O}_{4}, \mathrm{M}=430.62 \mathrm{~g} \cdot \mathrm{mol}^{-1}$

$\mathbf{R f}=0.26($ pentane/AcOEt 8:2)

$\mathbf{M p}=45^{\circ} \mathrm{C}$

${ }^{1} \mathbf{H}$ NMR $\left(300 \mathrm{MHz}, \mathrm{CDCl}_{3}\right) \delta 6.12(\mathrm{~d}, J=4.1 \mathrm{~Hz}, 1 \mathrm{H}), 5.96(\mathrm{dd}, J=10.6 \mathrm{~Hz}, J=4.1 \mathrm{~Hz}, 1 \mathrm{H})$, $5.68(\mathrm{t}, J=10.6 \mathrm{~Hz}, 1 \mathrm{H}), 4.84(\mathrm{~s}, 1 \mathrm{H}), 4.32(\mathrm{~s}, 2 \mathrm{H}), 2.78(\mathrm{t}, J=10.6 \mathrm{~Hz}, 1 \mathrm{H}), 2.57(\mathrm{~m}, 2 \mathrm{H}), 2.17-$ $1.18(\mathrm{~m}, 9 \mathrm{H}), 1.45(\mathrm{~s}, 1 \mathrm{H}: \mathrm{OH}), 1.37(\mathrm{~s}, 3 \mathrm{H}), 1.28(\mathrm{~s}, 3 \mathrm{H}), 1.14(\mathrm{~s}, 1 \mathrm{H}: \mathrm{OH}), 1.00(\mathrm{~d}, J=6.8 \mathrm{~Hz}$, $6 \mathrm{H}), 0.97(\mathrm{~d}, J=6.4 \mathrm{~Hz}, 2 \mathrm{H}), 0.96(\mathrm{~d}, J=6.8 \mathrm{~Hz}, 6 \mathrm{H}), 0.84(\mathrm{~d}, J=6.7 \mathrm{~Hz}, 2 \mathrm{H}) .{ }^{13} \mathbf{C}$ NMR $(50$ $\left.\mathrm{MHz}, \mathrm{CDCl}_{3}\right) \delta 158.3\left(\mathrm{C}_{\text {quat }}\right), 140.8\left(\mathrm{C}_{\text {quat }}\right), 139.4(+), 137.5\left(\mathrm{C}_{\text {quat }}\right), 127.6(+), 126.8(+), 114.8$ $\left(\mathrm{C}_{\text {quat }}\right), 91.3\left(\mathrm{C}_{\text {quat }}\right), 82.1(+), 78.7\left(\mathrm{C}_{\text {quat }}\right), 65.1(-), 49.7(+), 47.8(-), 44.1(-), 40.5(+), 36.7(-), 35.2$ $(-), 30.3(+), 29.7(-), 28.8(+), 25.6(+), 25.5(+), 25.0(+), 24.7(-), 24.6(+), 23.6(+), 22.8(+)$. IR $\left(\mathrm{CHCl}_{3}\right) \vee 3419,2951,2925,2867,2359,1449,1368,1316,1276,1243,1206,1137,1112,1065$, 1023, 997, 920, 851, 831, 774, 734, $628 \mathrm{~cm}^{-1}$. HRMS (ESI, positive ion): calc'd for $\left(\mathrm{C}_{27} \mathrm{H}_{42} \mathrm{O}_{4} \mathrm{Li}\right)^{+}$ 437.3238; found 437.3250. COSY, NOESY, HSQC, HMBC.

The stannane derivative (2-(tributylstannyl)ethynyl)trimethylsilane is commercially available.

The following products were prepared by literature methods:

- The vinyl stannanes ((E)-4-(tributylstannyl)-2-methylbut-3-en-2-ol, $\quad$ 1- $((E)-2-$ (tributylstannyl)vinyl)cyclohexanol $)^{3,4}$

- The vinyl iodides ((E)-4-iodo-2-methylbut-3-en-2-ol, $(E)$-1-iodooct-1-ene, (E)-1-iodo-4phenylbut-1-ene) ${ }^{5,6,7}$ The 1-((E)-2-iodovinyl)cyclohexanol has already been described in literature. $^{8}$

- These vinyl stannanes (1-[(E)-2-(tributylstannyl)vinyl $]$ cyclopentanol, (tributylstannyl)vinyl]-2,6-dimethylheptan-4-ol) and these vinyl iodides ((1-[(E)-2iodovinyl]cyclopentanol, 4-[(E)-2-iodovinyl]-2,6-dimethylheptan-4-ol, $\quad$ 1-[(E)-2iodovinyl]cyclohexanol) have already been described in our preceding paper. ${ }^{2 \mathrm{c}}$ 


\section{Computational Section}

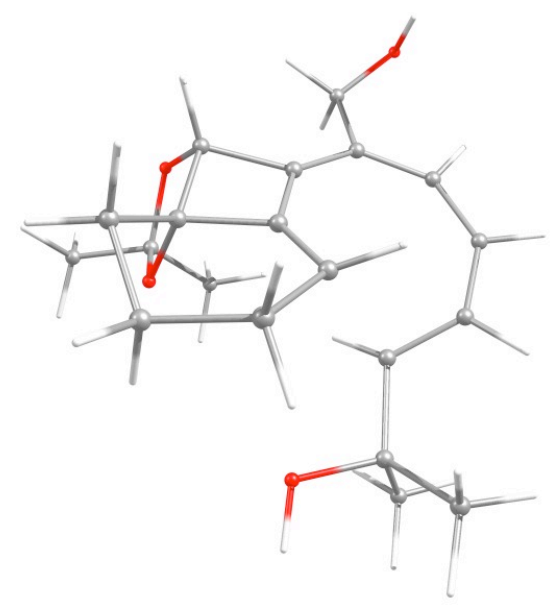

Pathway IIa: $\quad$ TS $^{\mathbf{1}}$ (9a to 15a)
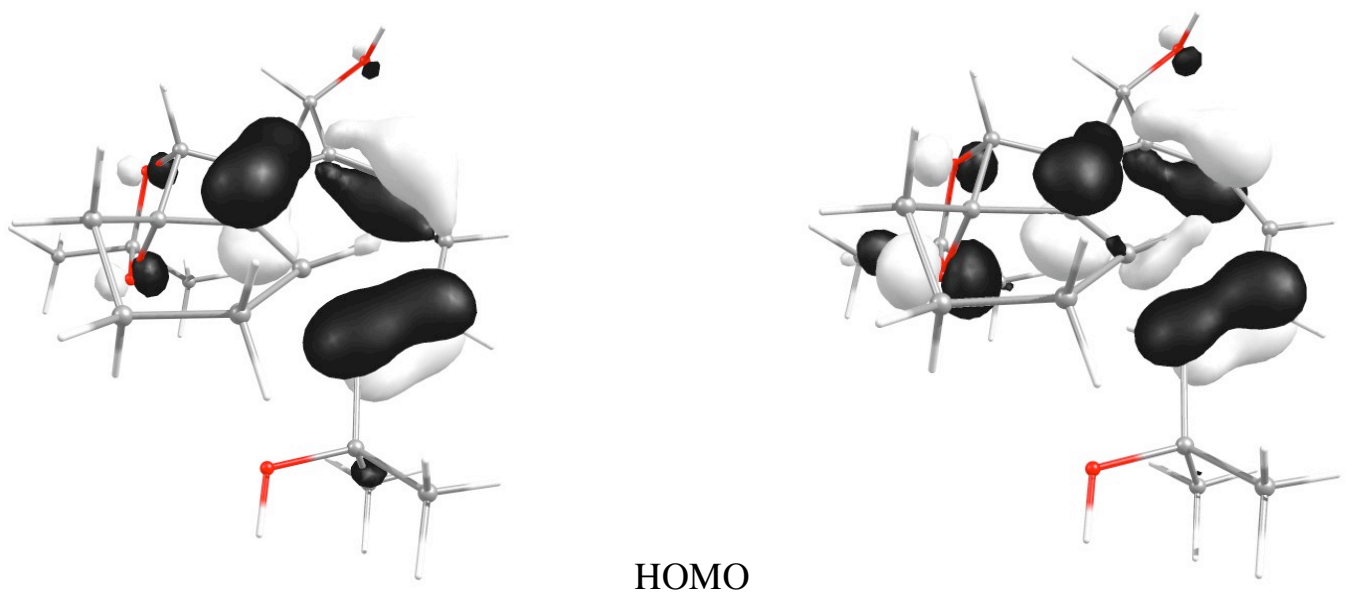

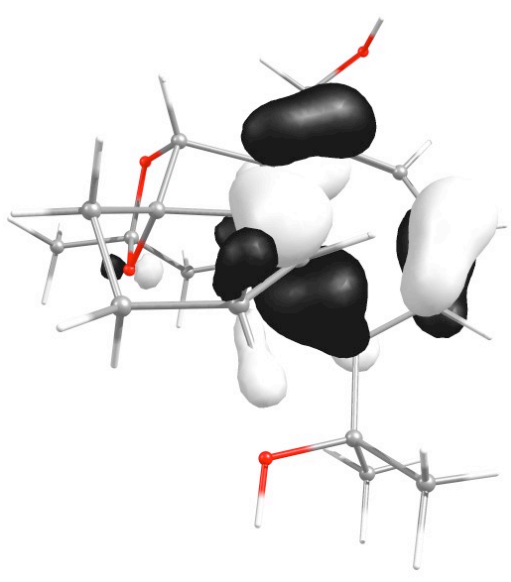

DFT
HOMO

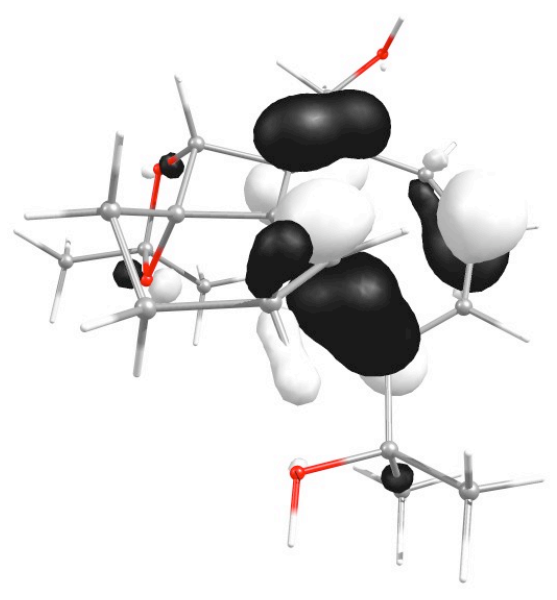

$\operatorname{CASSCF}(8,8)$ 


\section{Pathway I.}

$X=6(r, r)$

Table S2. B3PW 91/6-31g(d,p) Absolute energies [hartree], zero point vibrational energies [hartree], spin contaminations, and dipole moments [D].

\begin{tabular}{llllll}
\hline & $E^{0}$ & ZPVE & $E^{0}+$ ZPVE & $\left\langle S^{2}\right\rangle$ & Dipl. \\
\hline $\mathbf{8}$ & -1636.00622 & 0.51387 & -1635.49235 & 0.00 & 2.79 \\
TS $^{\mathbf{1}}\left(\mathbf{8} \rightarrow \mathbf{1 4}^{\prime}\right)$ & -1635.99570 & 0.51459 & -1635.48111 & 0.00 & 4.63 \\
$\mathbf{1 4}^{\prime}$ & -1636.03676 & 0.51775 & -1635.51901 & 0.00 & 3.81 \\
TS $^{\mathbf{2}}\left(\mathbf{1 4}^{\prime} \rightarrow \mathbf{2 0}^{\prime}\right)$ & -1636.00478 & 0.51711 & -1635.48767 & 0.00 & 2.81 \\
$\mathbf{2 0}^{\prime}$ & -1636.03255 & 0.51798 & -1635.51456 & 0.00 & 4.12 \\
\hline
\end{tabular}

$X=6(r, s)$

Table S3. B3PW 91/6-31g(d,p) Absolute energies [hartree], zero point vibrational energies [hartree], spin contaminations, and dipole moments [D].

\begin{tabular}{llllll}
\hline & $E^{0}$ & ZPVE & $E^{0}+$ ZPVE & $\left\langle S^{2}\right\rangle$ & Dipl. \\
\hline $\mathbf{8}$ & -1636.00622 & 0.51387 & -1635.49235 & 0.00 & 2.79 \\
TS $^{\mathbf{1}}(\mathbf{8} \rightarrow \mathbf{1 4})$ & -1635.99023 & 0.51438 & -1635.47585 & 0.00 & 3.94 \\
$\mathbf{1 4}$ & -1636.04605 & 0.51786 & -1635.52819 & 0.00 & 2.91 \\
TS $^{\mathbf{2}}(\mathbf{1 4} \rightarrow \mathbf{2 0})$ & -1636.00880 & 0.51678 & -1635.49202 & 0.00 & 4.31 \\
$\mathbf{2 0}$ & -1636.02304 & 0.51851 & -1635.50453 & 0.00 & 4.72 \\
\hline
\end{tabular}

8 $\mathrm{SiC} 23 \mathrm{H} 32 \mathrm{O} 6$

C1

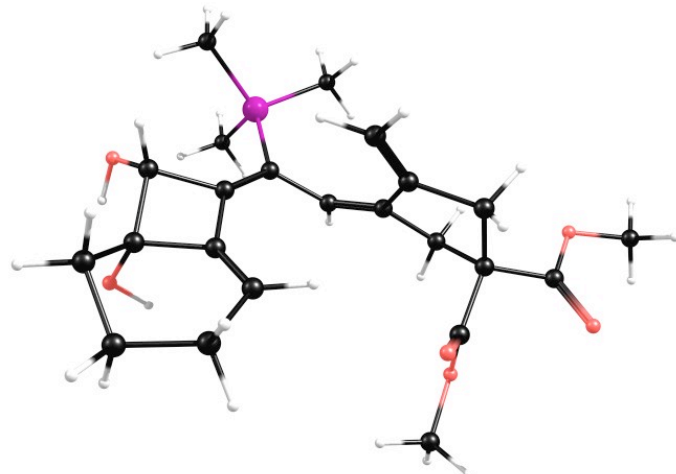

$\begin{array}{rrrr}6 & 3.755520000 & -0.228286000 & -0.376721000 \\ 6 & 2.268401000 & 0.071109000 & -0.069896000 \\ 8 & 3.907858000 & -1.898543000 & 1.371804000 \\ 8 & 4.763789000 & 0.393223000 & 0.363219000 \\ 6 & 1.588033000 & 1.201365000 & 0.219394000 \\ 6 & -0.271415000 & 0.369584000 & -2.403176000 \\ 6 & -1.167052000 & 0.476226000 & -1.411916000 \\ 6 & 0.182272000 & 1.178641000 & 0.662066000 \\ 6 & -0.951272000 & 0.887795000 & -0.010151000 \\ 1 & 0.035212000 & 1.529856000 & 1.688196000 \\ 1 & 3.977237000 & -0.110318000 & -1.444285000 \\ 1 & 0.778593000 & 0.588379000 & -2.251778000 \\ 6 & 1.993727000 & -1.376958000 & -0.089088000 \\ 6 & 1.042812000 & -2.250090000 & -0.455236000 \\ 6 & 1.399418000 & -3.687309000 & -0.722279000 \\ 6 & 2.866322000 & -4.053098000 & -0.433572000 \\ 6 & 3.848053000 & -2.929594000 & -0.804553000 \\ 6 & 3.460386000 & -1.716615000 & 0.014334000 \\ 1 & 0.727307000 & -4.353705000 & -0.164639000 \\ 1 & 1.164563000 & -3.880999000 & -1.780744000 \\ 1 & 2.989632000 & -4.257861000 & 0.636579000 \\ 1 & 3.120457000 & -4.979021000 & -0.960426000 \\ 1 & 3.773733000 & -2.687601000 & -1.872197000 \\ 1 & 4.881050000 & -3.229200000 & -0.598715000 \\ 1 & 0.026092000 & -1.934806000 & -0.671480000\end{array}$


$-0.134895000$

1.180084000

3.137894000

$-1.795780000$

1.943585000

$14 \quad 2.410112000$

2.928649000

0.256307000

$6 \quad 1.044854000$

4.200243000

$-0.058382000$

1.466469000

5.211603000

$-0.078021000$

0.553890000

4.024230000

$-1.021203000$

0.270968000

4.178312000

0.715489000

3.720466000

3.121761000

4.162125000

$-1.085473000$

4.064821000

2.482751000

(584551000

3.321496000

3.161295000

2.881779000

3.239046000

$-1.112309000$

$-0.889241000$

$-2.076574000$

3.549743000

2.424042000

3.989064000

4.261083000

3.106053000

2.546633000

0.060446000

0.196768000

$-2.648954000$

$-3.139761000$

1.061326000

$-2.840516000$

$-0.685029000$

0.997060000

2.008321000

0.768240000

0.022609000

0.301432000

$-3.199859000$

$-0.552209000$

$-5.552273000$

$-1.420535000$

$-2.992080000$

$-2.630080000$

$-2.340980000$

1.616945000

$-4.964204000$

$-1.535919000$

$-3.251360000$

1.950851000

3.037929000

$-6.397917000$

1.497296000

1.598320000

$-6.846370000$

$-2.862062000$

$-3.149439000$

$-2.779364000$

$-3.527889000$

$-3.850483000$

$-3.249086000$

1.962964000

2.036306000

2.761990000

2.139926000

$-3.398083000$

$-1.598362000$

$-2.061432000$

$-2.212801000$

0.646244000

0.519062000

1.713947000

$-0.165116000$

$-0.110431000$

$-0.055452000$

0.306716000

$-0.390046000$

$-0.187532000$

1.617417000

$-0.210785000$

$-0.272887000$

$-1.076016000$

0.697912000

2.144817000

3.202157000

1.636217000

2.019405000

$\mathrm{TS}^{1}(8 \rightarrow 14)$

$\mathrm{SiC} 23 \mathrm{H} 32 \mathrm{O} 6$

C1

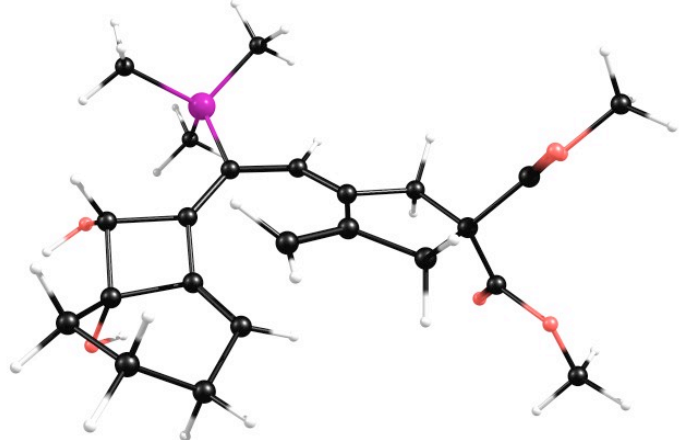

3.925348000

$-0.315414000$

$-0.132020000$

2.433298000

0.021454000

0.136726000

4.160759000

$-2.382501000$

1.192003000

4.806961000

0.153770000

0.857266000

1.748992000

1.206531000

0.148339000

0.110584000

$-1.313480000$

$-1.471214000$

$-0.861190000$

$-0.592222000$

$-0.803006000$

0.366245000

1.384044000

0.243652000

$-0.726356000$

0.600801000

$-0.070472000$

0.070854000

2.380926000

51170000

4.278345000

$-0.027643000$

$-1.149560000$

1.015542000

$-0.806101000$

$-1.839658000$

2.103832000

$-1.351135000$

0.243244000

1.031838000

$-2.215108000$

0.164977000

1.403388000

$-3.604635000$

$-0.260143000$ 


$\begin{array}{lrrr}6 & 2.424208000 & -3.592974000 & -1.388360000 \\ 6 & 3.710204000 & -2.835442000 & -1.084096000 \\ 6 & 3.543534000 & -1.841942000 & 0.043484000 \\ 1 & 1.828330000 & -4.155015000 & 0.622962000 \\ 1 & 0.486788000 & -4.154961000 & -0.597708000 \\ 1 & 2.682612000 & -4.652913000 & -1.648616000 \\ 1 & 1.941676000 & -3.136253000 & -2.294197000 \\ 1 & 4.050577000 & -2.293198000 & -2.004040000 \\ 1 & 4.516839000 & -3.565208000 & -0.803077000 \\ 1 & 0.125190000 & -2.107812000 & 0.779827000 \\ 1 & 5.506976000 & -0.510720000 & 0.936517000 \\ 1 & 3.869165000 & -1.854027000 & 1.948127000 \\ 14 & 2.621205000 & 2.820111000 & 0.140889000 \\ 6 & 1.496783000 & 4.192449000 & -0.321615000 \\ 1 & 2.081892000 & 5.066370000 & -0.691928000 \\ 1 & 0.789050000 & 3.902051000 & -1.131250000 \\ 1 & 0.889368000 & 4.546007000 & 0.542847000 \\ 6 & 3.991147000 & 2.805905000 & -1.074845000 \\ 1 & 4.377126000 & 3.834870000 & -1.257738000 \\ 1 & 4.843730000 & 2.187790000 & -0.705548000 \\ 1 & 3.675373000 & 2.388990000 & -2.057793000 \\ 6 & 3.299155000 & 3.139481000 & 1.807422000 \\ 1 & 3.838485000 & 4.112900000 & 1.853549000 \\ 1 & 2.503277000 & 3.161752000 & 2.585904000 \\ 1 & 4.024386000 & 2.339702000 & 2.092249000 \\ 1 & -0.180518000 & -2.219249000 & -2.025754000 \\ 6 & -2.274573000 & -1.098626000 & -0.752627000 \\ 1 & -2.673082000 & -1.275686000 & -1.785900000 \\ 1 & -2.326426000 & -2.068530000 & -0.193777000 \\ 6 & -2.082134000 & 1.070543000 & 0.399774000 \\ 1 & -2.342166000 & 2.059661000 & -0.056022000 \\ 1 & -2.089385000 & 1.193499000 & 1.514799000 \\ 6 & -3.097910000 & -0.010028000 & -0.030497000 \\ 6 & -4.129860000 & 0.587664000 & -0.962575000 \\ 8 & -4.344371000 & 0.318821000 & -2.144050000 \\ 6 & -3.797391000 & -0.603472000 & 1.172855000 \\ 8 & -3.605880000 & -0.370289000 & 2.365663000 \\ 8 & -4.883968000 & 1.562938000 & -0.370453000 \\ 8 & -4.742011000 & -1.534048000 & 0.836715000 \\ 6 & -5.883854000 & 2.181252000 & -1.183759000 \\ 1 & -6.362435000 & 2.918733000 & -0.493392000 \\ 1 & -5.404390000 & 2.685839000 & -2.056610000 \\ 1 & -6.614164000 & 1.414312000 & -1.536989000 \\ 6 & -5.455900000 & -2.140692000 & 1.916380000 \\ 1 & -6.164321000 & -2.836348000 & 1.402853000 \\ 1 & -4.745834000 & -2.691588000 & 2.578707000 \\ 1 & -5.997973000 & -1.358973000 & 2.500702000\end{array}$

14

$\mathrm{SiC} 23 \mathrm{H} 32 \mathrm{O} 6$

C1

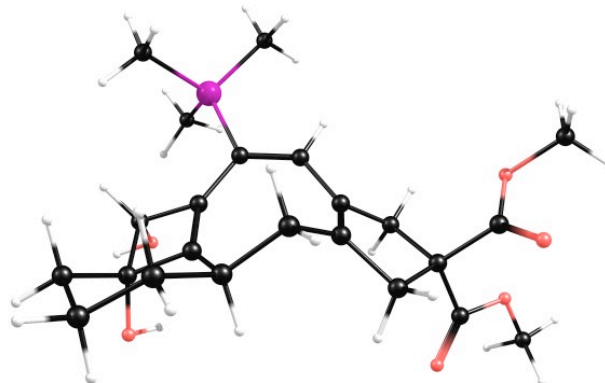

$\begin{array}{rrrr}6 & 3.496718000 & -0.367436000 & 1.006583000 \\ 6 & 2.211690000 & -0.017925000 & 0.266986000 \\ 8 & 3.069518000 & -2.747818000 & 1.620754000 \\ 8 & 3.399325000 & -0.193388000 & 2.412138000 \\ 6 & 1.594337000 & 1.289783000 & 0.051240000 \\ 6 & 0.157981000 & -1.184550000 & -1.806607000 \\ 6 & -0.875845000 & -0.657020000 & -0.863987000\end{array}$




\begin{tabular}{|c|c|c|c|}
\hline 6 & 0.252101000 & 1.472764000 & -0.094124000 \\
\hline 6 & -0.813691000 & 0.481202000 & -0.141046000 \\
\hline 1 & -0.109008000 & 2.502132000 & -0.13307000 \\
\hline 1 & 4.416851000 & 0.096656000 & 0.61844500 \\
\hline 1 & 0.690318000 & -0.349135000 & -2.277602000 \\
\hline 6 & 1.997172000 & -1.306979000 & -0.11287700 \\
\hline 6 & 1.190923000 & -2.074090000 & -1.09665200 \\
\hline 6 & 2.183598000 & -2.752970000 & -2.07053200 \\
\hline 6 & 3.340685000 & -3.464202000 & -1.35404500 \\
\hline 6 & 4.160910000 & -2.503773000 & -0.479533000 \\
\hline 6 & 3.244704000 & -1.849049000 & 0.546153000 \\
\hline 1 & 1.638441000 & -3.464102000 & -2.703365000 \\
\hline 1 & 2.591756000 & -1.982962000 & -2.741022000 \\
\hline 1 & 2.943369000 & -4.266591000 & -0.71978500 \\
\hline 1 & 3.990137000 & -3.942320000 & -2.09669500 \\
\hline 1 & 4.607964000 & -1.724833000 & -1.11169800 \\
\hline 1 & 4.975888000 & -3.030763000 & 0.02934000 \\
\hline 1 & 0.660579000 & -2.874471000 & -0.558308000 \\
\hline 1 & 4.220221000 & -0.527240000 & 2.796591000 \\
\hline 1 & 2.597592000 & -2.243274000 & 2.300006000 \\
\hline 14 & 2.704817000 & 2.837110000 & 0.056797000 \\
\hline 6 & 4.102701000 & 2.609226000 & -1.200385000 \\
\hline 1 & 4.721262000 & 3.512575000 & -1.249498000 \\
\hline 1 & 4.763770000 & 1.772075000 & -0.95551400 \\
\hline 1 & 3.698246000 & 2.429358000 & -2.2022410 \\
\hline 6 & 3.409695000 & 3.156400000 & 1.7792700 \\
\hline 1 & 4.204090000 & 3.910413000 & 1.7457650 \\
\hline 1 & 2.623520000 & 3.528030000 & 2.4453690 \\
\hline 1 & 3.806072000 & 2.242108000 & 2.2289680 \\
\hline 6 & 1.701462000 & 4.349188000 & -0.4748290 \\
\hline 1 & 2.363232000 & 5.221309000 & -0.52606200 \\
\hline 1 & 1.248832000 & 4.220033000 & -1.46327400 \\
\hline 1 & 0.903723000 & 4.590824000 & 0.2353310 \\
\hline 1 & -0.325822000 & -1.756948000 & -2.608 \\
\hline 6 & -2.159322000 & -1.402799000 & -0.6( \\
\hline 1 & -2.626178000 & -1.811170000 & -1.5026020 \\
\hline 1 & -1.998019000 & -2.239226000 & 0.092227 \\
\hline 6 & -2.074943000 & 0.677986000 & 0.6739490 \\
\hline 1 & -2.466473000 & 1.697491000 & 0.6414850 \\
\hline 1 & -1.876343000 & 0.441370000 & 1.7282600 \\
\hline 6 & -3.070948000 & -0.348527000 & $0.0648180 \mathrm{C}$ \\
\hline 6 & -3.994714000 & 0.253420000 & -1.00367000 \\
\hline 8 & -4.541092000 & -0.408764000 & -1.85783700 \\
\hline 6 & -3.934259000 & -0.959717000 & 1.16812300 \\
\hline 8 & -3.659100000 & -1.960008000 & 1.78860800 \\
\hline 8 & -4.154050000 & 1.579705000 & -0.88853900 \\
\hline 8 & -5.023333000 & -0.209870000 & 1.41106900 \\
\hline 6 & -5.052834000 & 2.163027000 & -1.83683900 \\
\hline 1 & -5.064242000 & 3.229871000 & -1.6147890 \\
\hline 1 & -4.703769000 & 1.985784000 & -2.8569520 \\
\hline 1 & -6.052886000 & 1.736608000 & -1.7274060 \\
\hline 6 & -5.866958000 & -0.684629000 & 2.4665950 \\
\hline 1 & -6.693899000 & 0.022578000 & 2.5233410 \\
\hline 1 & -6.234296000 & -1.688574000 & 2.241386 \\
\hline & & & \\
\hline
\end{tabular}

\section{$\mathrm{TS}^{2}(14 \rightarrow 20)$}

$\mathrm{SiC} 23 \mathrm{H} 32 \mathrm{O} 6$

C1 


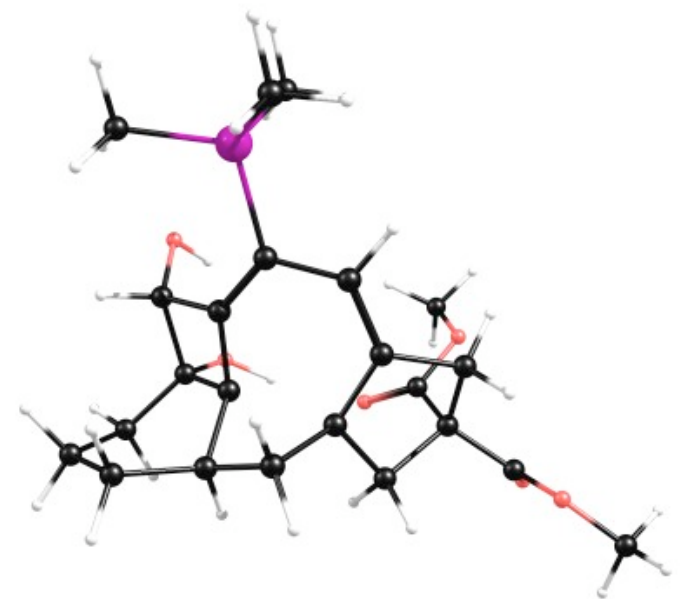

\begin{tabular}{lrrr}
6 & -2.634231000 & 0.923790000 & 0.888840000 \\
6 & -1.804584000 & 0.268038000 & -0.208123000 \\
8 & -0.797343000 & 1.710984000 & 2.256496000 \\
8 & -2.941537000 & 0.209902000 & 2.051799000 \\
6 & -1.760055000 & -1.037479000 & -0.671537000 \\
6 & -0.011804000 & 1.324855000 & -2.325238000 \\
6 & 0.729429000 & 0.543822000 & -1.243926000 \\
6 & -0.512055000 & -1.590363000 & -1.085141000 \\
6 & 0.665892000 & -0.874627000 & -1.132835000 \\
1 & -0.443336000 & -2.677675000 & -1.132776000 \\
1 & -3.554517000 & 1.381659000 & 0.506478000 \\
1 & -0.768862000 & 0.690242000 & -2.797248000 \\
6 & -0.871935000 & 1.365238000 & -0.242972000 \\
6 & -0.650813000 & 2.332469000 & -1.385990000 \\
6 & -1.910419000 & 3.126760000 & -1.714849000 \\
6 & -2.503691000 & 3.803071000 & -0.440785000 \\
6 & -1.791577000 & 3.453752000 & 0.884190000 \\
6 & -1.466176000 & 1.972738000 & 1.036479000 \\
1 & -1.696385000 & 3.880304000 & -2.482081000 \\
1 & -2.645770000 & 2.438715000 & -2.149855000 \\
1 & -2.483467000 & 4.891993000 & -0.554914000 \\
1 & -3.563902000 & 3.540478000 & -0.356928000 \\
1 & -2.399372000 & 3.784506000 & 1.734232000 \\
1 & -0.842864000 & 4.001727000 & 0.956119000 \\
1 & 0.133867000 & 3.036030000 & -1.070024000 \\
1 & -2.144871000 & 0.327820000 & 2.601037000 \\
1 & 0.129499000 & 1.485717000 & 2.073167000 \\
14 & -3.201100000 & -2.251677000 & -0.405673000 \\
6 & -3.270916000 & -3.363111000 & -1.938612000 \\
1 & -4.080984000 & -4.094702000 & -1.841406000 \\
1 & -3.458908000 & -2.777480000 & -2.844740000 \\
1 & -2.342245000 & -3.922532000 & -2.092592000 \\
6 & -4.850831000 & -1.353707000 & -0.242761000 \\
1 & -5.672536000 & -2.078766000 & -0.250451000 \\
1 & -4.901636000 & -0.797994000 & 0.697237000 \\
1 & -5.017420000 & -0.656850000 & -1.070923000 \\
6 & -2.893801000 & -3.317680000 & 1.123371000 \\
1 & -3.691364000 & -4.057804000 & 1.253088000 \\
1 & -1.943691000 & -3.858432000 & 1.056616000 \\
1 & -2.866208000 & -2.683536000 & 2.014187000 \\
1 & 0.646144000 & 1.724799000 & -3.106950000 \\
6 & 2.142841000 & 0.985428000 & -0.894942000 \\
1 & 2.713317000 & 1.173242000 & -1.814662000 \\
1 & 2.189644000 & 1.885729000 & -0.277722000 \\
6 & 2.045675000 & -1.433240000 & -0.853537000 \\
6 & 2.584588000 & -1.649670000 & -1.783927000 \\
\hline & 2.055788000 & -2.334476000 & -0.236975000 \\
\hline & 2.742041000 & -0.234844000 & -0.157046000 \\
\hline & 4.993550000 & -0.238736000 & -0.225760000 \\
\hline & 1.884499000 & 0.793335000 & 1.884226000
\end{tabular}




$\begin{array}{rrrr}8 & 4.708751000 & -0.575440000 & -1.447959000 \\ 8 & 2.617594000 & -1.325133000 & 1.946316000 \\ 6 & 6.132920000 & -0.544297000 & -1.603677000 \\ 1 & 6.322054000 & -0.835571000 & -2.636372000 \\ 1 & 6.518487000 & 0.459452000 & -1.409725000 \\ 1 & 6.608903000 & -1.244318000 & -0.913075000 \\ 6 & 2.334660000 & -1.346755000 & 3.351995000 \\ 1 & 2.601640000 & -2.347538000 & 3.688814000 \\ 1 & 2.933197000 & -0.593548000 & 3.868707000 \\ 1 & 1.275223000 & -1.150207000 & 3.531076000\end{array}$

\section{0}

$\mathrm{SiC} 23 \mathrm{H} 32 \mathrm{O} 6$

C1

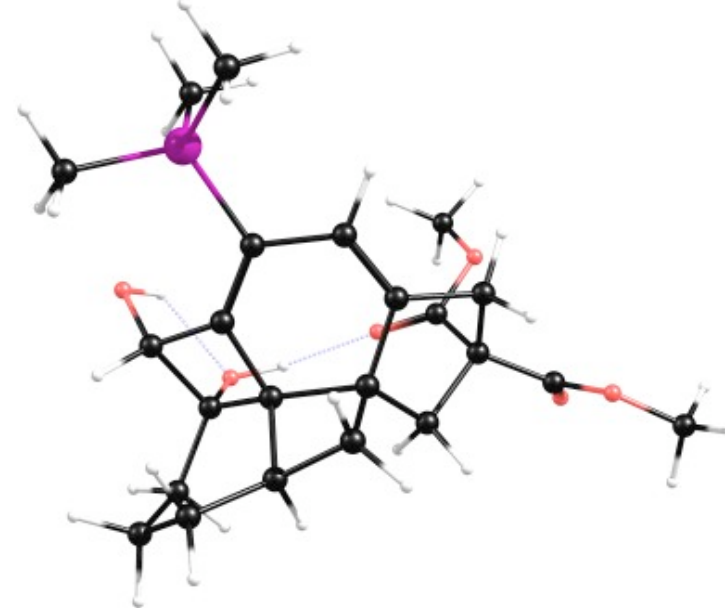

$\begin{array}{lrrr}6 & -2.650586000 & 1.005388000 & 0.852803000 \\ 6 & -1.865924000 & 0.392100000 & -0.289590000 \\ 8 & -0.780298000 & 1.157476000 & 2.403411000 \\ 8 & -3.151384000 & 0.168173000 & 1.853160000 \\ 6 & -1.816140000 & -0.874629000 & -0.772155000 \\ 6 & 0.120254000 & 1.746637000 & -2.081914000 \\ 6 & 0.578921000 & 0.828989000 & -0.894858000 \\ 6 & -0.520409000 & -1.378380000 & -1.241307000 \\ 6 & 0.602089000 & -0.635828000 & -1.195439000 \\ 1 & -0.443677000 & -2.436172000 & -1.488884000 \\ 1 & -3.446192000 & 1.691734000 & 0.543263000 \\ 1 & -0.567304000 & 1.199153000 & -2.733507000 \\ 6 & -0.731887000 & 1.370626000 & -0.151134000 \\ 6 & -0.616038000 & 2.609666000 & -1.051941000 \\ 6 & -1.865292000 & 3.451630000 & -1.165756000 \\ 6 & -2.196363000 & 3.991439000 & 0.261637000 \\ 6 & -1.467020000 & 3.271499000 & 1.427557000 \\ 6 & -1.315764000 & 1.761218000 & 1.249567000 \\ 1 & -1.734354000 & 4.285315000 & -1.865045000 \\ 1 & -2.689233000 & 2.839859000 & -1.553613000 \\ 1 & -1.933411000 & 5.053434000 & 0.316739000 \\ 1 & -3.278225000 & 3.949530000 & 0.424980000 \\ 1 & -2.008993000 & 3.449186000 & 2.363276000 \\ 1 & -0.466269000 & 3.698090000 & 1.572930000 \\ 1 & 0.158017000 & 3.235229000 & -0.580068000 \\ 1 & -2.380102000 & 0.028510000 & 2.434151000 \\ 1 & 0.066693000 & 0.726719000 & 2.196492000 \\ 14 & -3.262808000 & -2.096081000 & -0.641897000 \\ 6 & -3.231717000 & -3.147059000 & -2.220596000 \\ 1 & -4.070864000 & -3.852010000 & -2.219758000 \\ 1 & -3.323894000 & -2.523742000 & -3.116405000 \\ 1 & -2.313859000 & -3.736279000 & -2.318742000 \\ 6 & -4.921668000 & -1.205127000 & -0.562466000 \\ 1 & -5.741987000 & -1.928322000 & -0.633462000 \\ 1 & -5.027060000 & -0.663366000 & 0.380753000 \\ 1 & -5.033210000 & -0.494891000 & -1.388505000\end{array}$




$\begin{array}{rrrr}6 & -3.053033000 & -3.241234000 & 0.847319000 \\ 1 & -3.839555000 & -4.003952000 & 0.868072000 \\ 1 & -2.087836000 & -3.758602000 & 0.825679000 \\ 1 & -3.112476000 & -2.663787000 & 1.774000000 \\ 1 & 0.905613000 & 2.212911000 & -2.687213000 \\ 6 & 1.983716000 & 1.151367000 & -0.331290000 \\ 1 & 2.559876000 & 1.742612000 & -1.050966000 \\ 1 & 1.961720000 & 1.696970000 & 0.614344000 \\ 6 & 2.031507000 & -1.091332000 & -1.267259000 \\ 1 & 2.503048000 & -0.818186000 & -2.219301000 \\ 1 & 2.175128000 & -2.162885000 & -1.110882000 \\ 6 & 2.675989000 & -0.226262000 & -0.150951000 \\ 6 & 4.188515000 & -0.070162000 & -0.202289000 \\ 8 & 4.887499000 & 0.082732000 & 0.774246000 \\ 6 & 2.316442000 & -0.823284000 & 1.210506000 \\ 8 & 1.608863000 & -0.302158000 & 2.049717000 \\ 8 & 4.654296000 & -0.055008000 & -1.462047000 \\ 8 & 2.856123000 & -2.031790000 & 1.366538000 \\ 6 & 6.064883000 & 0.167335000 & -1.582373000 \\ 1 & 6.275919000 & 0.145544000 & -2.651046000 \\ 1 & 6.338713000 & 1.134998000 & -1.155205000 \\ 1 & 6.620796000 & -0.617088000 & -1.063730000 \\ 6 & 2.591075000 & -2.674301000 & 2.621087000 \\ 1 & 3.103080000 & -3.634360000 & 2.571320000 \\ 1 & 2.982381000 & -2.072164000 & 3.443802000 \\ 1 & 1.516729000 & -2.816023000 & 2.757945000\end{array}$

$\mathrm{TS}^{1}\left(8 \rightarrow 14^{\prime}\right)$

$\mathrm{SiC} 23 \mathrm{H} 32 \mathrm{O} 6$

C1

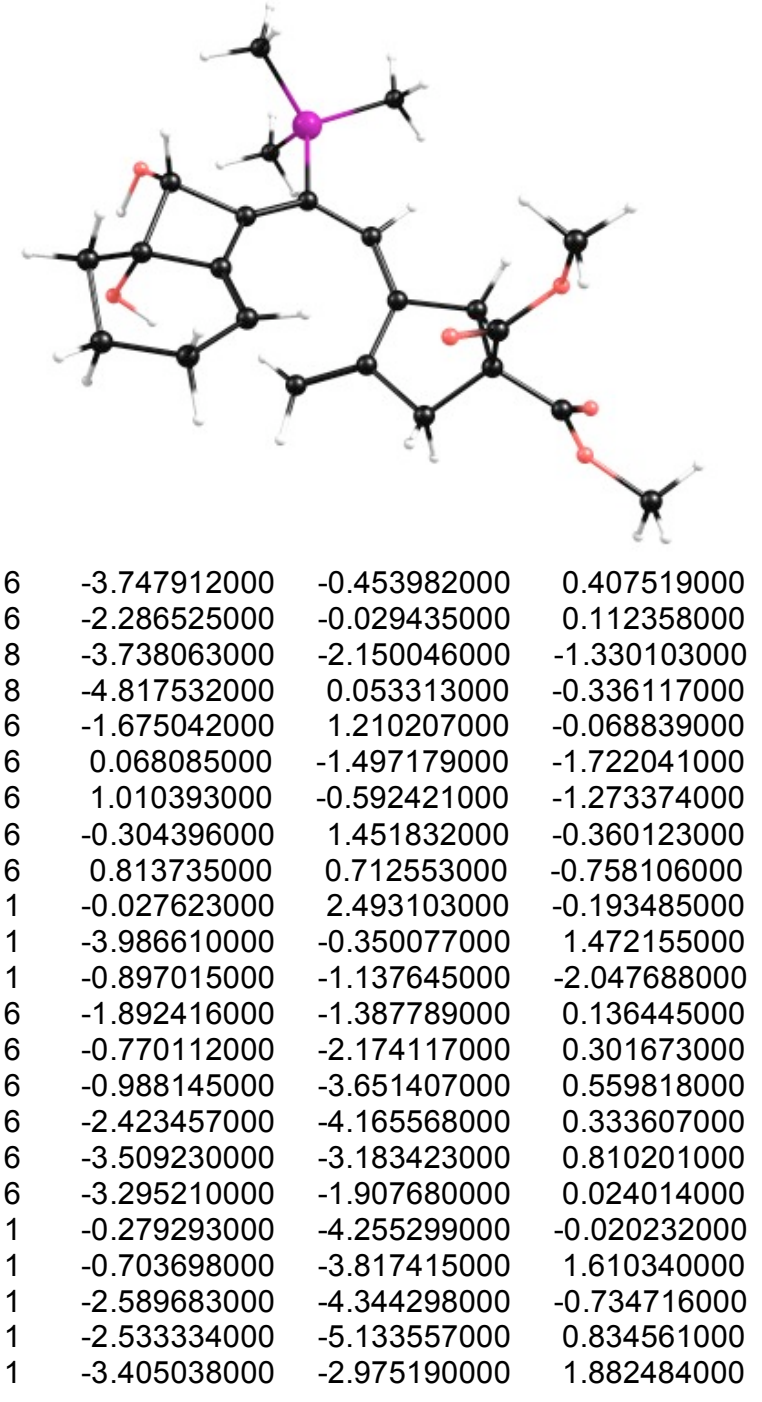


$-1.811518000$

$-1.923995000$

$14-2.752323000$

2.788392000

$-1.127276000$

$6-1.636388000$

4.283797000

0.111800000

$-0.980388000$

4.528607000

0.447629000

$-2.269901000$

5.160222000

4.146245000

$-0.394045000$

$-1.011875000$

2.665655000

$-3.965013000$

3.648206000

626760000

1.337023000

1.555505000

$-4.774601000$

1.964156000

1.736323000

$-4.774601000$

2.355843000

$-3.696649000$

3.094814000

1.339721000

2.480984000

$-1.494314000$

$-3.018050000$

3.169271000

2.270890000

$-2.350579000$

$-4.393281000$

4.025773000

$-427207200$

0.395956000

$-2.436968000$

$-1.670921000$

$-1.438438000$

2.185487000

1.364911000

1.868237000

2.49174900

2.099147000

$-2.160167000$

$-0.623108000$

$-1.549087000$

0.183234000

2486399000

$-0.945366000$

$-1.246317000$

2.900126000

$-0.932260000$

$-2.263496000$

2.675805000

$-1.933634000$

0.169738000

$-0.818565000$

3.132709000

$-0.231336000$

$-0.398303000$

3.118879000

$-1.242191000$

1.077681000

2.633770000

0.461170000

533536000

4.558415000

4.901304000

1.389436000

$-0.849288000$

3.698113000

0.718353000

$-1.543020000$

1.830802000

5.396370000

$-0.499371000$

$-0.418061000$

$6.761991000 \quad-0.344892000$

$7.294691000-1.190314000$

$6.845818000 \quad-0.357598000$

$-0.819898000$

$-0.385489000$

7.167138000

0.598312000

$-1.909338000$

3.723348000

0.454214000

$-0.445631000$

4.222065000

1.311132000

3.237744000

2.707481000

0.352177000

3.689536000

4.276146000

$-0.465255000$

3.626898000

3.444936000

14'

$\mathrm{SiC} 23 \mathrm{H} 32 \mathrm{O} 6$

$\mathrm{C} 1$

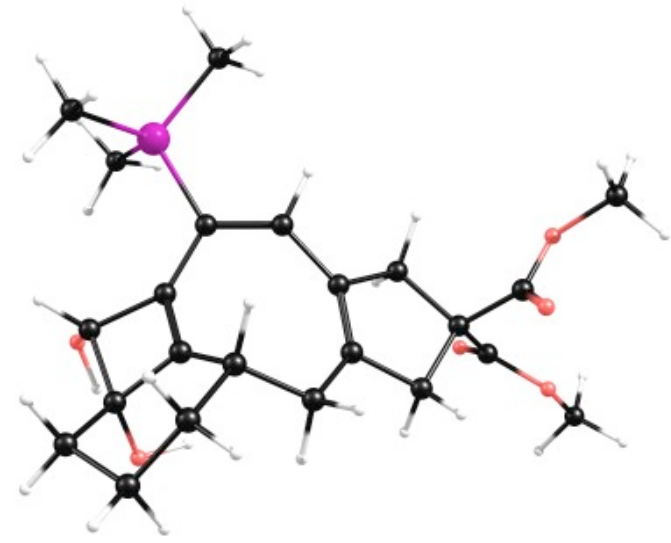

2.403105000

2.580696000

3.784091000

1.764675000

$-0.041762000$

$-0.852510000$

0.425433000

$-0.656577000$

0.017136000

4.577029000
$-0.472601000$

0.039123000

$-2.581585000$

$-0.265055000$

1.299126000

$-2.077137000$

$-0.977068000$

1.331978000

0.350436000

2.315134000

$-0.180297000$
0.974223000

0.209762000

1.685122000

2.347389000

$-0.096297000$

$-1.030639000$

$-0.403193000$

$-0.359418000$

$-0.183291000$

$-0.600768000$

0.480320000 


\begin{tabular}{|c|c|c|c|}
\hline 1 & -0.610577000 & -2.454269000 & -1.895331000 \\
\hline 6 & 2.141346000 & -1.184567000 & -0.299274000 \\
\hline 6 & 1.380151000 & -1.707269000 & -1.459111000 \\
\hline 6 & 2.177998000 & -2.879893000 & -2.089691000 \\
\hline 6 & 2.969497000 & -3.743056000 & -1.088968000 \\
\hline & 3.957269000 & -2.918151000 & -0.244180000 \\
\hline & 3.143644000 & -1.909712000 & 0.544020000 \\
\hline & 1.498778000 & -3.515203000 & -2.671322000 \\
\hline & 2.890486000 & -2.455775000 & -2.809054000 \\
\hline 1 & 2.284807000 & -4.262235000 & -0.406500000 \\
\hline 1 & 3.505159000 & -4.522231000 & -1.643030000 \\
\hline 1 & 4.661161000 & -2.388208000 & -0.898776000 \\
\hline 1 & 4.541331000 & -3.560412000 & 0.423487000 \\
\hline 1 & 1.286782000 & -0.901396000 & -2.199679000 \\
\hline 1 & 3.342948000 & -1.028774000 & 2.756247000 \\
\hline 1 & 1.689587000 & -2.226141000 & 1.799586000 \\
\hline 14 & 2.780799000 & 2.902974000 & -0.069127000 \\
\hline 6 & 4.295671000 & 2.712031000 & -1.185774000 \\
\hline 1 & 4.881547000 & 3.637937000 & -1.197505000 \\
\hline 1 & 4.957269000 & 1.910352000 & -0.842704000 \\
\hline 1 & 4.005172000 & 2.487979000 & -2.217603000 \\
\hline 6 & 3.322727000 & 3.238196000 & 1.706721000 \\
\hline 1 & 2.465325000 & 3.530612000 & 2.322344000 \\
\hline 1 & 3.764694000 & 2.346731000 & 2.162617000 \\
\hline 1 & 4.057351000 & 4.050177000 & 1.747940000 \\
\hline 6 & 1.737324000 & 4.343761000 & -0.706246000 \\
\hline 1 & 2.341689000 & 5.257751000 & -0.713756000 \\
\hline 1 & 1.382173000 & 4.176635000 & -1.728517000 \\
\hline 1 & 0.866792000 & 4.535081000 & -0.070269000 \\
\hline 1 & 0.002259000 & -2.924568000 & -0.329473000 \\
\hline 6 & -2.242757000 & -1.399803000 & 0.024724000 \\
\hline 1 & -2.729446000 & -2.047588000 & -0.711120000 \\
\hline 1 & -2.192447000 & -1.958882000 & 0.970456000 \\
\hline 6 & -1.918482000 & 0.968359000 & 0.411235000 \\
\hline 1 & -2.171431000 & 1.931375000 & -0.044492000 \\
\hline 1 & -1.797011000 & 1.154047000 & 1.485475000 \\
\hline 6 & -3.024841000 & -0.081596000 & 0.198477000 \\
\hline 6 & -3.813024000 & 0.220538000 & -1.079002000 \\
\hline 8 & -3.835602000 & -0.458091000 & -2.078579000 \\
\hline 6 & -3.985143000 & -0.160198000 & 1.381233000 \\
\hline 8 & -3.794322000 & 0.309389000 & 2.478455000 \\
\hline 8 & -4.480616000 & 1.382349000 & -0.952873000 \\
\hline 8 & -5.070871000 & -0.886866000 & 1.059808000 \\
\hline 6 & -5.258862000 & 1.764693000 & -2.091405000 \\
\hline 1 & -5.730713000 & 2.709468000 & -1.822732000 \\
\hline 1 & -4.620352000 & 1.890999000 & -2.969169000 \\
\hline 1 & -6.014618000 & 1.006592000 & -2.310955000 \\
\hline 6 & -6.024160000 & -1.061360000 & 2.113752000 \\
\hline 1 & -6.831818000 & -1.654179000 & 1.685436000 \\
\hline 1 & -5.571005000 & -1.584864000 & 2.959131000 \\
\hline 1 & -6.397664000 & -0.093592000 & 2.456965000 \\
\hline
\end{tabular}




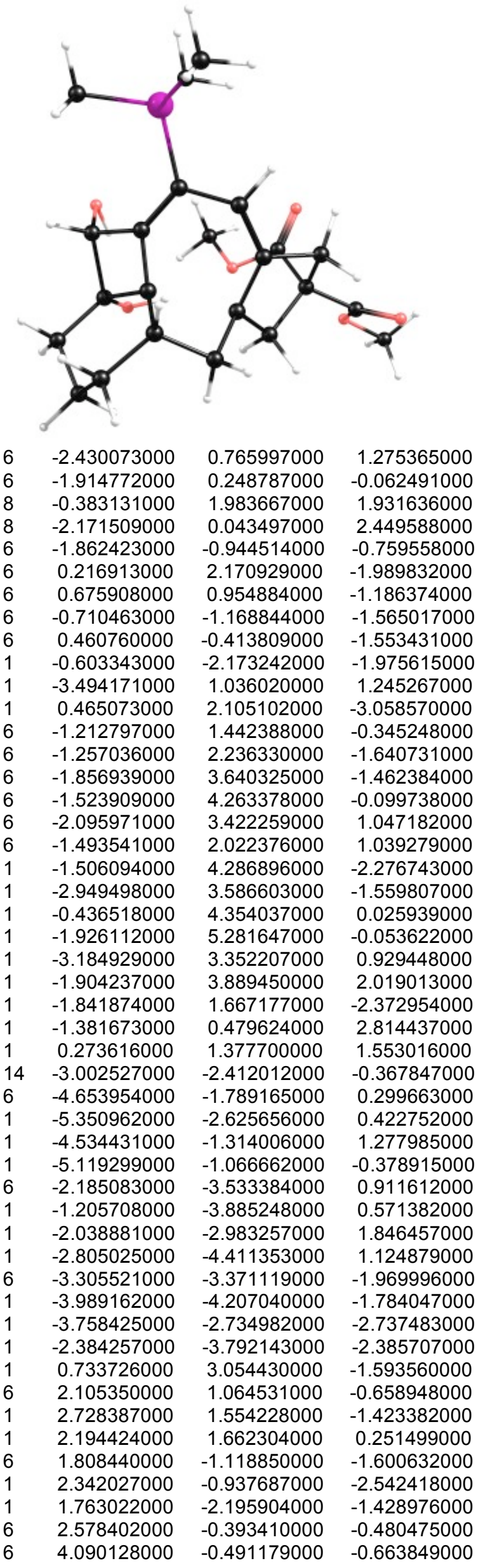


20'

$\mathrm{SiC} 23 \mathrm{H} 32 \mathrm{O} 6$

C1

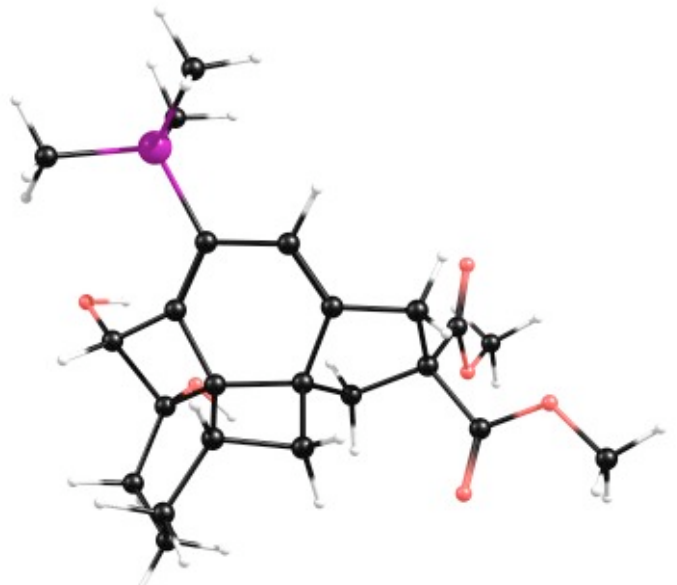

$6 \quad-2.920011000$

$6-2.146087000$

$-1.088858000$

$-3.621814000$

$-2.143965000$

0.695679000

0.422459000

$-0.852009000$

0.297471000

$-0.807492000$

$-3.598413000$

1.042948000

$-0.982463000$

$-0.781534000$

$-1.041796000$

$-0.657421000$

$-1.528478000$

$-1.551728000$

$-0.483919000$

$-2.104882000$

0.402768000

$-0.759274000$

$-2.554233000$

$-1.192590000$

$-1.357372000$

$-2.941659000$

$-0.185620000$

$14-3.606207000$

$6 \quad-5.239135000$

$1-6.083437000$

$1-5.318234000$

$1-5.336245000$

$6-3.474259000$

$1-2.509197000$

$1-3.578066000$
1.253705000 0.378285000 1.305727000 0.701917000 $-0.890096000$ 1.810400000 0.698167000 $-1.372531000$ $-0.681105000$ $-2.390060000$ 1.947286000 1.480198000 1.324209000 2.260071000 3.748459000 4.189525000 3.494469000 1.964313000 4.325168000 3.974275000 3.965574000 5.276089000 3.876775000 3.748846000 1.895005000 0.338851000 1.592184000 $-2.082296000$ $-1.142183000$ $-1.839204000$ $-0.457092000$ $-0.562818000$ $-3.062495000$ $-3.573659000$ $-2.398296000$
0.775862000 $-0.186485000$ 2.278108000 1.849512000 $-0.638519000$ $-1.522830000$ $-0.469178000$ $-1.172398000$ $-1.069878000$ $-1.556726000$ 0.262640000 $-2.506797000$ $-0.205006000$ $-1.440831000$ $-1.218430000$ 0.194476000 1.243850000 1.104942000 $-1.967557000$ $-1.383207000$ 0.377116000 0.294597000 1.165122000 2.256184000 $-2.297778000$ 2.436571000 2.451256000 $-0.470403000$ $-0.523098000$ $-0.476564000$ 0.325697000 $-1.447310000$ 1.141150000 1.221399000 2.004575000 


$\begin{array}{rr}-3.821185000 & 1.204330000 \\ -3.290524000 & -1.927677000 \\ -3.949729000 & -1.905916000 \\ -2.763050000 & -2.887458000 \\ -3.930120000 & -1.903676000 \\ 2.561199000 & -1.150151000 \\ 0.452406000 & 0.587014000 \\ 1.355695000 & 0.910740000 \\ -0.034668000 & 1.456051000 \\ -1.204472000 & -1.263722000 \\ -0.895721000 & -2.229041000 \\ -2.292397000 & -1.202867000 \\ -0.539199000 & -0.098064000 \\ 0.200793000 & -0.636783000 \\ 1.398683000 & -0.637568000 \\ -1.587004000 & 0.909560000 \\ -2.761776000 & 0.904724000 \\ -0.672456000 & -1.156565000 \\ -1.006174000 & 1.858695000 \\ -0.090792000 & -1.712663000 \\ -0.926788000 & -2.080902000 \\ 0.589229000 & -2.529820000 \\ 0.464214000 & -0.948746000 \\ -1.887766000 & 2.879660000 \\ -1.268475000 & 3.545992000 \\ -2.337842000 & 3.422698000 \\ -2.684536000 & 2.444242000\end{array}$

\section{Pathway II.}

$X=6(r, r)$ IIa

Table S4. B3PW 91/6-31g(d,p) Absolute energies [hartree], zero point vibrational energies [hartree], spin contaminations, and dipole moments [D].

\begin{tabular}{llllll}
\hline & $E^{U}$ & ZPVE & $E^{U}+$ ZPVE & $\left\langle S^{2}\right\rangle$ & Dipl. \\
\hline $\mathbf{9 a}$ & -1079.41179 & 0.44672 & -1078.96507 & 0.00 & 3.96 \\
$\mathbf{T S}^{\mathbf{1}}(\mathbf{9 a} \rightarrow \mathbf{1 5 a})$ & -1079.39974 & 0.44751 & -1078.95223 & 0.00 & 3.69 \\
$\mathbf{1 5 a}$ & -1079.42852 & 0.45087 & -1078.97764 & 0.00 & 3.68 \\
$\mathbf{T S}^{2}(\mathbf{1 5 a} \rightarrow \mathbf{2 1 a})$ & -1079.40124 & 0.44871 & -1078.95252 & 0.00 & 2.59 \\
$\mathbf{2 1 a}$ & -1079.43884 & 0.45044 & -1078.98841 & 0.00 & 2.00 \\
\hline
\end{tabular}

$X=6(r, s)$ IIb

Table S5. B3PW 91/6-31g(d,p) Absolute energies [hartree], zero point vibrational energies [hartree], spin contaminations, and dipole moments [D].

\begin{tabular}{llllll}
\hline & $E^{0}$ & ZPVE & $E^{0}+$ ZPVE & $\left\langle S^{2}\right\rangle$ & Dipl. \\
\hline $\mathbf{1 0 a}$ & -1079.41179 & 0.446723 & -1078.96507 & 0.00 & 3.96 \\
$\mathbf{T S}^{1}(\mathbf{1 0 a} \rightarrow \mathbf{1 6 a})$ & -1079.39829 & 0.447415 & -1078.95087 & 0.00 & 4.71 \\
$\mathbf{1 6 a}$ & -1079.44178 & 0.450663 & -1078.99111 & 0.00 & 4.03 \\
$\mathbf{T S}^{2}(\mathbf{1 6 a} \rightarrow \mathbf{2 2 a})$ & -1079.40776 & 0.448784 & -1078.95897 & 0.00 & 3.65 \\
$\mathbf{2 2 a}$ & -1079.42685 & 0.450544 & -1078.97631 & 0.00 & 3.45 \\
\hline
\end{tabular}

$X=7(r, r) \quad$ IId

Table S6. B3PW 91/6-31g(d,p) Absolute energies [hartree], zero point vibrational energies [hartree], spin contaminations, and dipole moments [D].

\begin{tabular}{llllll}
\hline & $E^{U}$ & ZPVE & $E^{0}+$ ZPVE & $\left\langle S^{2}\right\rangle$ & Dipl. \\
\hline $\mathbf{1 1 a}$ & -1118.71159 & 0.47570 & -1118.23590 & 0.00 & 3.80 \\
$\mathbf{T S}^{1}(\mathbf{1 1 a} \rightarrow \mathbf{1 7 a})$ & -1118.69694 & 0.47648 & -1118.22046 & 0.00 & 3.32 \\
$\mathbf{1 7 a}$ & -1118.72626 & 0.47989 & -1118.24638 & 0.00 & 4.33 \\
$\mathbf{T S}^{2}(\mathbf{1 7 a} \rightarrow \mathbf{2 3 a})$ & -1118.69421 & 0.47763 & -1118.21658 & 0.00 & 4.05 \\
$\mathbf{2 3 a}$ & -1118.73047 & 0.47916 & -1118.25131 & 0.00 & 4.04 \\
\hline
\end{tabular}


$X=7(r, s) \quad$ IIe

Table S7. B3PW 91/6-31g(d,p) Absolute energies [hartree], zero point vibrational energies [hartree], spin contaminations, and dipole moments [D].

\begin{tabular}{llllll}
\hline & $E^{U}$ & ZPVE & $E^{0}+$ ZPVE & $\left\langle S^{2}\right\rangle$ & Dipl. \\
\hline $\mathbf{1 2 a}$ & -1118.71159 & 0.47569 & -1118.23589 & 0.00 & 3.80 \\
$\mathbf{T S}^{1}(\mathbf{1 2 a} \rightarrow \mathbf{1 8 a})$ & -1118.70216 & 0.47681 & -1118.22535 & 0.00 & 4.53 \\
$\mathbf{1 8 a}$ & -1118.74121 & 0.47929 & -1118.26192 & 0.00 & 3.64 \\
$\mathbf{T S}^{\mathbf{2}}(\mathbf{1 8 a} \rightarrow \mathbf{2 4 a})$ & -1118.71303 & 0.47749 & -1118.23554 & 0.00 & 3.06 \\
$\mathbf{2 4 a}$ & -1118.73248 & 0.47942 & -1118.25306 & 0.00 & 3.17 \\
\hline
\end{tabular}

$X=4(r, r)$

Table S8. B3PW 91/6-31g(d,p) Absolute energies [hartree], zero point vibrational energies [hartree], spin contaminations, and dipole moments [D].

\begin{tabular}{llllll}
\hline & $E^{0}$ & ZPVE & $E^{0}+$ ZPVE & $\left\langle S^{2}\right\rangle$ & Dipl. \\
\hline t4 & -1000.72533 & 0.38677 & -1000.33856 & 0.00 & 3.37 \\
TS $^{1}$ & -1000.71092 & 0.38694 & -1000.32398 & 0.00 & 3.65 \\
c4 $^{2}$ & -1000.74322 & 0.39027 & -1000.35296 & 0.00 & 3.55 \\
TS $^{2}$ & -1000.73115 & 0.38891 & -1000.34225 & 0.00 & 2.70 \\
$\mathbf{f 4}$ & -1000.79724 & 0.39148 & -1000.40576 & 0.00 & 2.34 \\
\hline
\end{tabular}

\section{$X=4(r, s)$}

Table S9. B3PW 91/6-31g(d,p) Absolute energies [hartree], zero point vibrational energies [hartree], spin contaminations, and dipole moments [D].

\begin{tabular}{llllll}
\hline & $E^{0}$ & ZPVE & $E^{0}+$ ZPVE & $\left\langle S^{2}\right\rangle$ & Dipl. \\
\hline $\mathbf{t 4}$ & -1000.72533 & 0.38677 & -1000.3385 & 0.00 & 3.37 \\
TS $^{1}$ & -1000.71092 & 0.38694 & -1000.3234 & 0.00 & 3.65 \\
c4 $^{2}$ & -1000.77067 & 0.39074 & -1000.3799 & 0.00 & 3.08 \\
TS $^{\mathbf{f 4}}$ & -1000.73390 & 0.38913 & -1000.3448 & 0.00 & 2.66 \\
$\mathbf{f}$ & $--^{a}$ & $-{ }^{a}$ & $-{ }^{a}$ & $-{ }^{a}$ & $-{ }^{a}$ \\
\hline
\end{tabular}

${ }^{a}$ The data are not available because of bond dissociation upon optimization.

$X=5(r, r)$

Table S10. B3PW 91/6-31g(d,p) Absolute energies [hartree], zero point vibrational energies [hartree], spin contaminations, and dipole moments [D].

\begin{tabular}{llllll}
\hline & $E^{0}$ & ZPVE & $E^{0}+$ ZPVE & $\left\langle S^{2}\right\rangle$ & Dipl. \\
\hline t5 & -1040.08857 & 0.41679 & -1039.67178 & 0.00 & 3.91 \\
TS $^{1}$ & -1040.07429 & 0.41781 & -1039.65648 & 0.00 & 3.71 \\
c5 & -1040.11114 & 0.42066 & -1039.69048 & 0.00 & 3.77 \\
TS $^{2}$ & -1040.08763 & 0.41900 & -1039.66863 & 0.00 & 2.59 \\
f5 & -1040.13559 & 0.42124 & -1039.71435 & 0.00 & 1.97 \\
\hline
\end{tabular}

\section{$X=8(r, r)$}

Table S11. B3PW 91/6-31g(d,p) Absolute energies [hartree], zero point vibrational energies [hartree], spin contaminations, and dipole moments [D].

\begin{tabular}{llllll}
\hline & $E^{0}$ & ZPVE & $E^{0}+$ ZPVE & $\left\langle S^{2}\right\rangle$ & Dipl. \\
\hline t8 & -1158.01153 & 0.50431 & -1157.50721 & 0.00 & 3.42 \\
TS $^{1}$ & -1157.99641 & 0.50526 & -1157.49114 & 0.00 & 3.17 \\
c8 $^{2}$ & -1158.02453 & 0.50910 & -1157.51542 & 0.00 & 2.64 \\
TS $^{2}$ & -1157.99497 & 0.50701 & -1157.48795 & 0.00 & 3.17 \\
$\mathbf{f 8}$ & -1158.02862 & 0.50854 & -1157.52007 & 0.00 & 1.90 \\
\hline
\end{tabular}

\section{$X=8(r, s)$}

Table S12. B3PW 91/6-31g(d,p) Absolute energies [hartree], zero point vibrational energies [hartree], spin contaminations, and dipole moments [D]. 


\begin{tabular}{llllll}
\hline & $E^{0}$ & ZPVE & $E^{0}+$ ZPVE & $\left\langle S^{2}\right\rangle$ & Dipl. \\
\hline t8 & -1158.01152 & 0.50431 & -1157.50721 & 0.00 & 3.42 \\
TS $^{1}$ & -1157.99022 & 0.50562 & -1157.48460 & 0.00 & 3.64 \\
c8 $^{\mathbf{T}}$ & -1158.03331 & 0.50886 & -1157.52444 & 0.00 & 4.14 \\
f8 $^{2}$ & -1158.00108 & 0.50608 & -1157.49500 & 0.00 & 3.62 \\
\hline
\end{tabular}

IIa.

$9 a$

$\mathrm{C} 2 \mathrm{OH} 28 \mathrm{O} 4$

C1

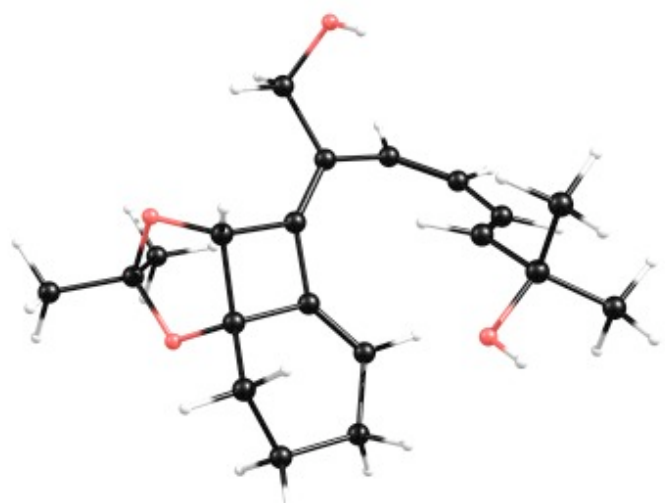

$\begin{array}{rrrr}6 & 1.593092000 & 0.214521000 & -1.066658000 \\ 6 & 0.653637000 & 0.764761000 & 0.029102000 \\ 8 & 2.871058000 & -1.293637000 & 0.176010000 \\ 8 & 2.940907000 & 0.616115000 & -1.084230000 \\ 6 & 3.634342000 & -0.117621000 & -0.069474000 \\ 6 & 4.985495000 & -0.526753000 & -0.633601000 \\ 1 & 5.583696000 & 0.358339000 & -0.865679000 \\ 1 & 5.527973000 & -1.136609000 & 0.093425000 \\ 1 & 4.838118000 & -1.107160000 & -1.546733000 \\ 6 & 3.767644000 & 0.708877000 & 1.207634000 \\ 1 & 4.334317000 & 1.622998000 & 1.008102000 \\ 1 & 2.784827000 & 0.980509000 & 1.596582000 \\ 1 & 4.294303000 & 0.129017000 & 1.970957000 \\ 6 & 0.219141000 & 2.027939000 & 0.265755000 \\ 6 & -2.709990000 & 0.322116000 & 0.024501000 \\ 6 & -2.838551000 & 1.058717000 & 1.142862000 \\ 6 & -0.678370000 & 2.451871000 & 1.336552000 \\ 6 & -1.909154000 & 2.024249000 & 1.713753000 \\ 6 & -3.754437000 & -0.590076000 & -0.570005000 \\ 6 & -4.877759000 & -0.966333000 & 0.396440000 \\ 1 & -4.470164000 & -1.437984000 & 1.294265000 \\ 1 & -5.472058000 & -0.096527000 & 0.692118000 \\ 1 & -5.559226000 & -1.676661000 & -0.085327000 \\ 6 & -4.327466000 & 0.067458000 & -1.836731000 \\ 1 & -3.523858000 & 0.296479000 & -2.542456000 \\ 1 & -5.035867000 & -0.608257000 & -2.333109000 \\ 1 & -4.856863000 & 0.994512000 & -1.598259000 \\ 8 & -3.026981000 & -1.771116000 & -0.943877000 \\ 1 & -3.641416000 & -2.343628000 & -1.419849000 \\ 6 & 0.820129000 & 3.169483000 & -0.528749000 \\ 1 & 1.531151000 & 3.715386000 & 0.105606000 \\ 1 & 1.396247000 & 2.778149000 & -1.378203000 \\ 8 & -0.134721000 & 4.136727000 & -0.932031000 \\ 1 & -0.842108000 & 3.666785000 & -1.388362000 \\ 1 & -0.316676000 & 3.331616000 & 1.872696000 \\ 1 & -2.323848000 & 2.553211000 & 2.571208000 \\ 1 & 1.196799000 & 0.276010000 & -2.085476000\end{array}$


$-0.568108000$

0.597925000

1.334820000

1.250298000

0.338805000

$-0.886858000$

$-0.343367000$

2.252688000

0.869140000

0.906104000

0.021387000

$-1.480253000$

$-1.523608000$

1.706472000

1.909667000

\section{$\mathrm{TS}^{1}(9 \mathrm{a} \rightarrow 15 \mathrm{a})$}

\section{$\mathrm{C} 2 \mathrm{H} 28 \mathrm{O} 4$}

C1

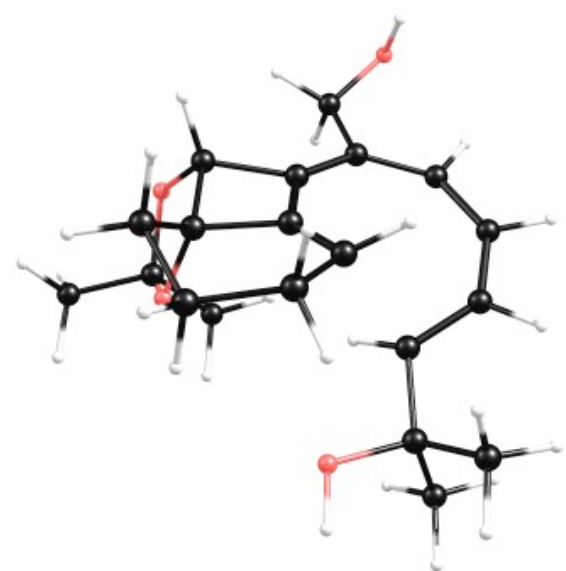

$\begin{array}{rrrr}6 & 2.205755000 & -0.587670000 & -0.738798000 \\ 6 & 1.280832000 & 0.642860000 & -0.646782000 \\ 8 & 0.829719000 & -2.039415000 & 0.445363000 \\ 8 & 2.898338000 & -1.034474000 & 0.399482000 \\ 6 & 1.964230000 & -1.706530000 & 1.247076000 \\ 6 & 2.615210000 & -2.992990000 & 1.733206000 \\ 1 & 3.508370000 & -2.769083000 & 2.322969000 \\ 1 & 1.915753000 & -3.558320000 & 2.354749000 \\ 1 & 2.901835000 & -3.604055000 & 0.874490000 \\ 6 & 1.539038000 & -0.803573000 & 2.401017000 \\ 1 & 2.399578000 & -0.568945000 & 3.034406000 \\ 1 & 1.121077000 & 0.130348000 & 2.020241000 \\ 1 & 0.782256000 & -1.307265000 & 3.009076000 \\ 6 & 1.534504000 & 1.966489000 & -0.293263000 \\ 6 & -1.893496000 & 0.861138000 & 0.404949000 \\ 6 & -1.863311000 & 2.221925000 & 0.087635000 \\ 6 & 0.607121000 & 3.005999000 & -0.110752000 \\ 6 & -0.790022000 & 3.108603000 & -0.060314000 \\ 6 & -3.200345000 & 0.190007000 & 0.800111000 \\ 6 & -3.508222000 & 0.551880000 & 2.264001000 \\ 1 & -2.679971000 & 0.248789000 & 2.910344000 \\ 1 & -3.667819000 & 1.626982000 & 2.383941000 \\ 1 & -4.415809000 & 0.034468000 & 2.602203000 \\ 6 & -4.386534000 & 0.558129000 & -0.097063000 \\ 1 & -4.163169000 & 0.357657000 & -1.147690000 \\ 1 & -5.260885000 & -0.038554000 & 0.187142000 \\ 1 & -4.670313000 & 1.609721000 & 0.002345000 \\ 8 & -2.948352000 & -1.214922000 & 0.715464000 \\ 1 & -3.710364000 & -1.665326000 & 1.099042000 \\ 6 & 3.003109000 & 2.329490000 & -0.068293000 \\ 1 & 3.244567000 & 2.246315000 & 0.997508000 \\ 1 & 3.646793000 & 1.607203000 & -0.589306000 \\ 8 & 3.336407000 & 3.658529000 & -0.425290000 \\ 1 & 3.134919000 & 3.760183000 & -1.363260000\end{array}$


$-1.110358000$ 0.016161000 $-1.016898000$

$-1.742902000$

$-1.220507000$

$-1.521562000$

$-1.033307000$

$-2.547085000$

$-2.134875000$

0.500964000

$-2.453363000$

1813602000

0.911277000

$-1.441050000$

$-2.794031000$

$-1.302769000$

$-1.739035000$

$-1.058587000$

$-1.298881000$

$-2.850908000$

$-1.409424000$

$-3.320936000$

0.808954000

$-2.104869000$

0.967434000

$-3.427091000$

$-2.831437000$

2.684514000

$-1.381908000$

0.957500000

$-1.900301000$

$-0.851557000$

$-1.844277000$

$-3.224707000$

$-0.795786000$

$-2.492446000$

$-2.895016000$

$-1.719811000$

$-0.104467000$

$-1.983621000$

\section{$15 a$}

$\mathrm{C} 2 \mathrm{OH} 28 \mathrm{O} 4$

C1

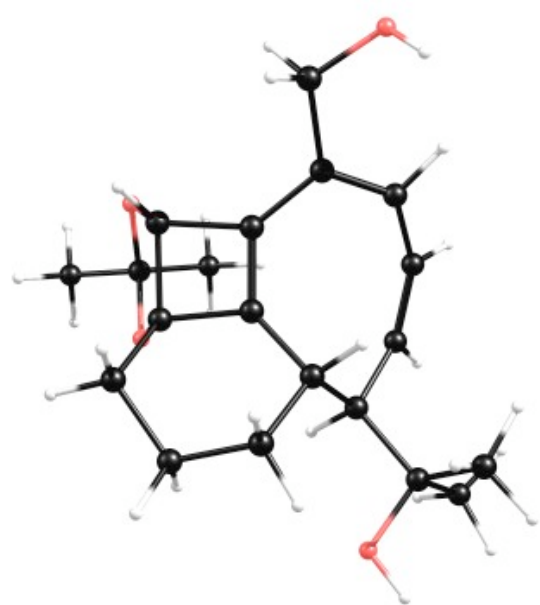

$\begin{array}{rrrr}6 & 2.378816000 & -0.194519000 & -0.776218000 \\ 6 & 1.267853000 & 0.831924000 & -0.506808000 \\ 8 & 1.498511000 & -2.080555000 & 0.312196000 \\ 8 & 3.238359000 & -0.576858000 & 0.266257000 \\ 6 & 2.552580000 & -1.518911000 & 1.099008000 \\ 6 & 3.529448000 & -2.632288000 & 1.441266000 \\ 1 & 4.371586000 & -2.233879000 & 2.013331000 \\ 1 & 3.031819000 & -3.401601000 & 2.037621000 \\ 1 & 3.905481000 & -3.082589000 & 0.520177000 \\ 6 & 1.996919000 & -0.836625000 & 2.345452000 \\ 1 & 2.814029000 & -0.393809000 & 2.922508000 \\ 1 & 1.286627000 & -0.052737000 & 2.078924000 \\ 1 & 1.484933000 & -1.572224000 & 2.973114000 \\ 6 & 1.137767000 & 2.248804000 & -0.269566000 \\ 6 & -1.911435000 & -0.176302000 & 0.503915000 \\ 6 & -1.480014000 & 0.888176000 & 1.491324000 \\ 6 & 0.150000000 & 2.728296000 & 0.528335000 \\ 6 & -0.747870000 & 2.027265000 & 1.447792000 \\ 6 & -3.475320000 & -0.222945000 & 0.419484000 \\ 6 & -4.135931000 & -0.196979000 & 1.804776000 \\ 1 & -3.691971000 & -0.949132000 & 2.464467000 \\ 1 & -4.074241000 & 0.782050000 & 2.286854000 \\ 1 & -5.200622000 & -0.436500000 & 1.695664000 \\ 6 & -4.040680000 & 0.908911000 & -0.442143000 \\ 1 & -3.721526000 & 0.814098000 & -1.482909000 \\ 1 & -5.137236000 & 0.893623000 & -0.423808000 \\ 1 & -3.715373000 & 1.884039000 & -0.065686000 \\ 8 & -3.770043000 & -1.497329000 & -0.167516000 \\ 1 & -4.729169000 & -1.556719000 & -0.254757000 \\ 6 & 2.154370000 & 3.176921000 & -0.901876000\end{array}$


3.124522000 2.299809000

1.858525000

1.044309000

0.161130000

$-0.817519000$

2.972867000

$-1.638053000$

0.288983000

$-1.177124000$

$-1.492192000$

$-0.686712000$

0.830501000

1.233872000

$-2.557645000$

$-1.251264000$

$-0.918423000$

$-1.005667000$

1.066026000

1.390794000

$-1.932719000$

$-1.408323000$
3.031036000 2.887808000 4.550634000 4.710112000 3.808380000 2.593360000 $-0.041319000$ $-1.149795000$ $-0.086977000$ $-0.028725000$ $-1.043104000$ $-2.351147000$ $-2.111438000$ $-1.256013000$ $-1.272755000$ $-0.544182000$ $-2.891475000$ $-3.000470000$ $-1.578499000$ $-3.051990000$ 0.730349000 0.981742000
$-0.410090000$ $-1.956292000$ $-0.785120000$ $-1.277210000$ 0.675296000 2.380494000 $-1.683864000$ 0.936601000 $-0.619359000$ $-0.849696000$ $-1.985382000$ $-1.922821000$ $-2.003728000$ $-0.816876000$ $-1.995977000$ $-2.934856000$ $-0.996479000$ $-2.746485000$ $-2.934669000$ $-2.003699000$ 2.468253000 $-1.214349000$

$\mathrm{TS}^{2}(15 \mathrm{a} \rightarrow 21 \mathrm{a})$ $\mathrm{C} 2 \mathrm{H} 28 \mathrm{O} 4$

C1

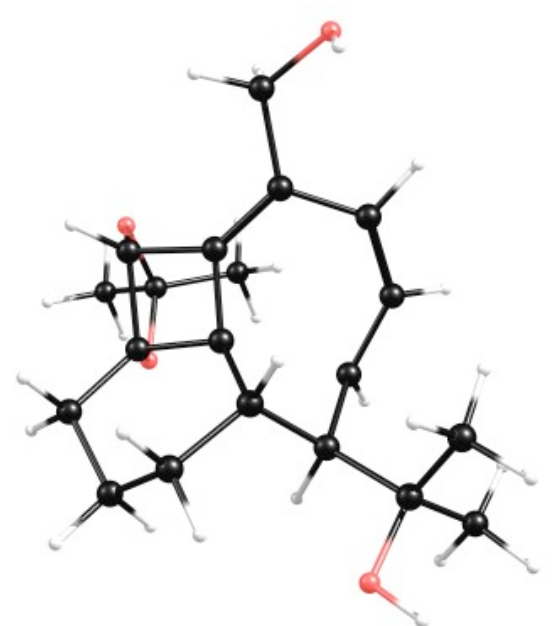

$\begin{array}{rrrr}6 & 2.172855000 & -0.141390000 & -0.993726000 \\ 6 & 1.001043000 & 0.790403000 & -0.711013000 \\ 8 & 1.830008000 & -1.805607000 & 0.616518000 \\ 8 & 3.273222000 & -0.171500000 & -0.117597000 \\ 6 & 2.916006000 & -0.963305000 & 1.018860000 \\ 6 & 4.100502000 & -1.852973000 & 1.359545000 \\ 1 & 4.964876000 & -1.243840000 & 1.636835000 \\ 1 & 3.849977000 & -2.509559000 & 2.196881000 \\ 1 & 4.358406000 & -2.464193000 & 0.492112000 \\ 6 & 2.507497000 & -0.071213000 & 2.186517000 \\ 1 & 3.354180000 & 0.550028000 & 2.493095000 \\ 1 & 1.676829000 & 0.578858000 & 1.906847000 \\ 1 & 2.199115000 & -0.686824000 & 3.036543000 \\ 6 & 0.777496000 & 2.141941000 & -0.524553000 \\ 6 & -2.006748000 & -0.521233000 & 0.405472000 \\ 6 & -1.054176000 & 0.273490000 & 1.303287000 \\ 6 & -0.200740000 & 2.527686000 & 0.417570000 \\ 6 & -0.823653000 & 1.671782000 & 1.327424000 \\ 6 & -3.509613000 & -0.173724000 & 0.514562000 \\ 6 & -3.960001000 & -0.209755000 & 1.978428000 \\ 1 & -3.710560000 & -1.173905000 & 2.432163000 \\ 1 & -3.493678000 & 0.586178000 & 2.566787000 \\ 1 & -5.046564000 & -0.075775000 & 2.043093000 \\ 6 & -3.876288000 & 1.166256000 & -0.123864000 \\ 1 & -3.688987000 & 1.148544000 & -1.200703000\end{array}$



$-4.942158000$
1.377607000
0.028593000
$-3.306791000$
1.989899000
0.315284000
$-4.154990000$
$-1.237588000$
$-5.099532000$
$-1.041245000$
$-0.200017000$
1.752680000
3.189681000
$-0.216241000$
2.595664000
3.278706000
$-1.029169000$
2.173846000
2.865110000
.334004000
1.193429000
4.485649000
$-1.992763000$
0.446888000
4.432078000
$-1.120754000$
$-0.217675000$
3.590710000
$-0.973901000$
2.139163000
$-0.149626000$
$-1.729304000$
0.651825000
2.542073000
$-1.563267000$
2.305314000
$-2.025023000$
$-1.937535000$
$-0.264908000$
.744420000
0.117430000
$-0.432378000$
$-1.455234000$
$-1.627243000$
$-0.422721000$
$-0.581436000$
$-2.821714000$
0.907651000
$-2.462334000$
$-0.937630000$
$-1.892344000$
$-1.492510000$
1.236409000
$-1.318305000$
$-1.530321000$
$-2.511181000$
$-1.911509000$
$-1.171743000$
$-1.317405000$
$-0.573875000$
$-1.923066000$
$-2.908023000$
$-0.850203000$
$-3.172461000$
$-0.775450000$
$-0.487317000$
1.169597000
$-3.660885000$
$-2.170313000$
1.535017000
$-2.161486000$
$-2.553570000$
$-1.010638000$
$-3.321961000$
$-1.274369000$
$-0.149554000$
2.312351000
$-1.583592000$
0.494389000
$-1.452275000$

21a

$\mathrm{C} 2 \mathrm{OH} 28 \mathrm{O} 4$

C1

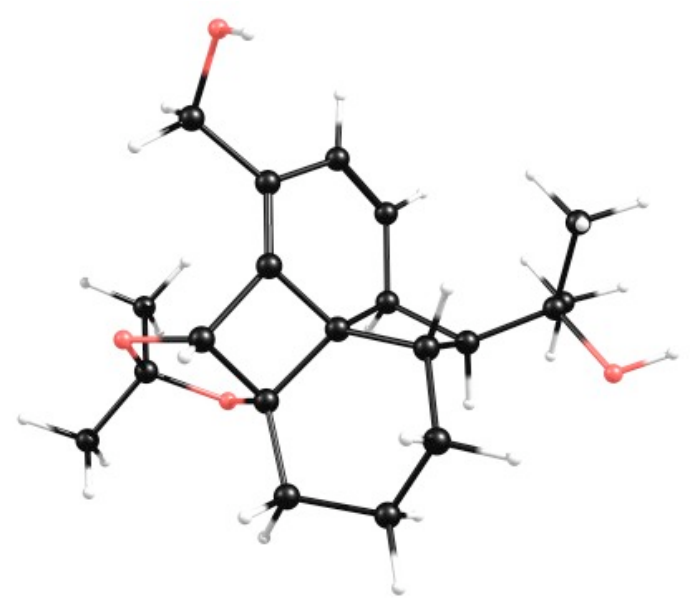

1.903675000

$-0.331702000$

$-1.083102000$

0.915455000

0.753259000

.

1.751094000

$-1.400802000$

0.962994000

3.178937000

$-0.322852000$

$-0.492810000$

3.075838000

$-0.878108000$

0.823434000

4.091293000

$-2.008109000$

0.932849000

5.105644000

$-1.622086000$

0.799069000

4.022946000

$-2.485262000$

1.914126000

3.896890000

$-2.755894000$

0.160272000

3.284623000

0.200731000

1.876748000

4.277826000

0.645438000

1.769977000

2.528286000

0.979700000

1.764960000

3.199528000

$-0.230539000$

2.878165000

0.935290000

2.054741000

$-2.172454000$

$-0.484746000$

$-0.386848000$

$-0.932837000$

0.195998000

2.533635000

0.370566000

1.040362000

$-0.142459000$

1.695997000

0.493075000

$-0.944601000$

0.043063000

1.177947000

$-3.611479000$

0.148958000

0.454184000

$-4.052181000$

$-0.806034000$

1.918254000

2.430644000 

$-3.500520000$
0.924090000
2.458576000
$-5.117792000$
0.401298000
1.978439000
$-3.854764000$
1.360028000
$-0.284995000$
$-3.674262000$
1.237317000
$-1.356013000$
$-4.897940000$
1.674670000
$-0.154913000$
$-3.213983000$
2.165151000
0.082341000
$-4.371371000$
$-0.995077000$
$-0.187107000$
$-5.287153000$
$-0.693821000$
$-0.231465000$
2.042889000
3.006484000
$-0.751186000$
2.556392000
3.357678000
0.151785000
2.791100000
2.493280000
$-1.370918000$
1.548468000
4.183140000
$-1.377780000$
1.057221000
3.899536000
3.605997000
$-2.157882000$
$-0.206250000$
2.117893000
0.661712000
$-1.618309000$
2.005089000
$-0.549682000$
1.922085000
$-2.152035000$
0.769501000
$-0.097185000$
$-1.504085000$
$-0.225744000$
$-0.200550000$
$-1.412526000$
$-0.521620000$
$-0.979833000$
$-1.468962000$
$-1.809725000$
$-0.786315000$
$-2.971202000$
$-1.794774000$
$-1.071454000$
0.703538000
$-2.709552000$
1.026667000
$-1.320657000$
$-0.827171000$
$-0.255811000$
$-2.519269000$
$-2.048995000$
$-1.994884000$
$-0.983621000$
$-1.655370000$
$-2.769553000$
$-1.290085000$
$-3.156042000$
$-0.114651000$
$-0.899755000$
$-3.894348000$
$-1.651239000$
$-1.772254000$
1.105722000
$-2.836789000$
$-0.132804000$
$-0.657448000$
$-3.453938000$
2.002965000
$-1.674483000$
0.340784000
$-1.602423000$

Ilb.

$10 a$

$\mathrm{C} 2 \mathrm{OH} 28 \mathrm{O} 4$

C1

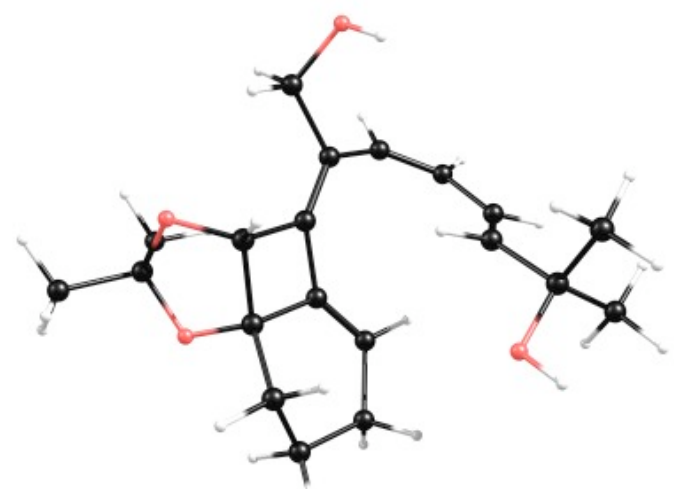

$\begin{array}{rrrr}6 & 1.593092000 & 0.214521000 & -1.066658000 \\ 6 & 0.653637000 & 0.764761000 & 0.029102000 \\ 8 & 2.871058000 & -1.293637000 & 0.176010000 \\ 8 & 2.940907000 & 0.616115000 & -1.084230000 \\ 6 & 3.634342000 & -0.117621000 & -0.069474000 \\ 6 & 4.985495000 & -0.526753000 & -0.633601000 \\ 1 & 5.583696000 & 0.358339000 & -0.865679000 \\ 1 & 5.527973000 & -1.136609000 & 0.093425000 \\ 1 & 4.838118000 & -1.107160000 & -1.546733000 \\ 6 & 3.767644000 & 0.708877000 & 1.207634000 \\ 1 & 4.334317000 & 1.622998000 & 1.008102000 \\ 1 & 2.784827000 & 0.980509000 & 1.596582000 \\ 1 & 4.294303000 & 0.129017000 & 1.970957000 \\ 6 & 0.219141000 & 2.027939000 & 0.265755000 \\ 6 & -2.709990000 & 0.322116000 & 0.024501000 \\ 6 & -2.838551000 & 1.058717000 & 1.142862000\end{array}$




$\begin{array}{rrrr}6 & -0.678370000 & 2.451871000 & 1.336552000 \\ 6 & -1.909154000 & 2.024249000 & 1.713753000 \\ 6 & -3.754437000 & -0.590076000 & -0.570005000 \\ 6 & -4.877759000 & -0.966333000 & 0.396440000 \\ 1 & -4.470164000 & -1.437984000 & 1.294265000 \\ 1 & -5.472058000 & -0.096527000 & 0.692118000 \\ 1 & -5.559226000 & -1.676661000 & -0.085327000 \\ 6 & -4.327466000 & 0.067458000 & -1.836731000 \\ 1 & -3.523858000 & 0.296479000 & -2.542456000 \\ 1 & -5.035867000 & -0.608257000 & -2.333109000 \\ 1 & -4.856863000 & 0.994512000 & -1.598259000 \\ 8 & -3.026981000 & -1.771116000 & -0.943877000 \\ 1 & -3.641416000 & -2.343628000 & -1.419849000 \\ 6 & 0.820129000 & 3.169483000 & -0.528749000 \\ 1 & 1.531151000 & 3.715386000 & 0.105606000 \\ 1 & 1.396247000 & 2.778149000 & -1.378203000 \\ 8 & -0.134721000 & 4.136727000 & -0.932031000 \\ 1 & -0.842108000 & 3.666785000 & -1.388362000 \\ 1 & -0.316676000 & 3.331616000 & 1.872696000 \\ 1 & -2.323848000 & 2.553211000 & 2.571208000 \\ 1 & 1.196799000 & 0.276010000 & -2.085476000 \\ 1 & -1.802078000 & 0.384266000 & -0.568108000 \\ 6 & 0.509757000 & -0.574652000 & 0.597925000 \\ 6 & -0.339078000 & -1.309454000 & 1.334820000 \\ 6 & -0.302858000 & -2.810008000 & 1.250298000 \\ 6 & 0.799712000 & -3.383166000 & 0.338805000 \\ 6 & 1.090283000 & -2.497350000 & -0.886858000 \\ 6 & 1.547346000 & -1.159942000 & -0.343367000 \\ 1 & -0.228699000 & -3.254034000 & 2.252688000 \\ 1 & -1.291665000 & -3.105855000 & 0.869140000 \\ 1 & 1.731840000 & -3.482124000 & 0.906104000 \\ 1 & 0.514748000 & -4.392307000 & 0.021387000 \\ 1 & 0.178186000 & -2.359463000 & -1.480253000 \\ 1 & 1.857488000 & -2.950052000 & -1.523608000 \\ 1 & -3.765870000 & 0.965235000 & 1.706472000 \\ 1 & -1.138891000 & -0.854376000 & 1.909667000 \\ & & & \\ \text { TS } & (10 \mathrm{a} \rightarrow 16 a) & & \\ \mathrm{C} 20 \mathrm{H} 2804 & & & \\ \mathrm{C} 1 & & & \\ & & & \\ 1 & & & \\ 1 & & & \\ 1 & & & \end{array}$

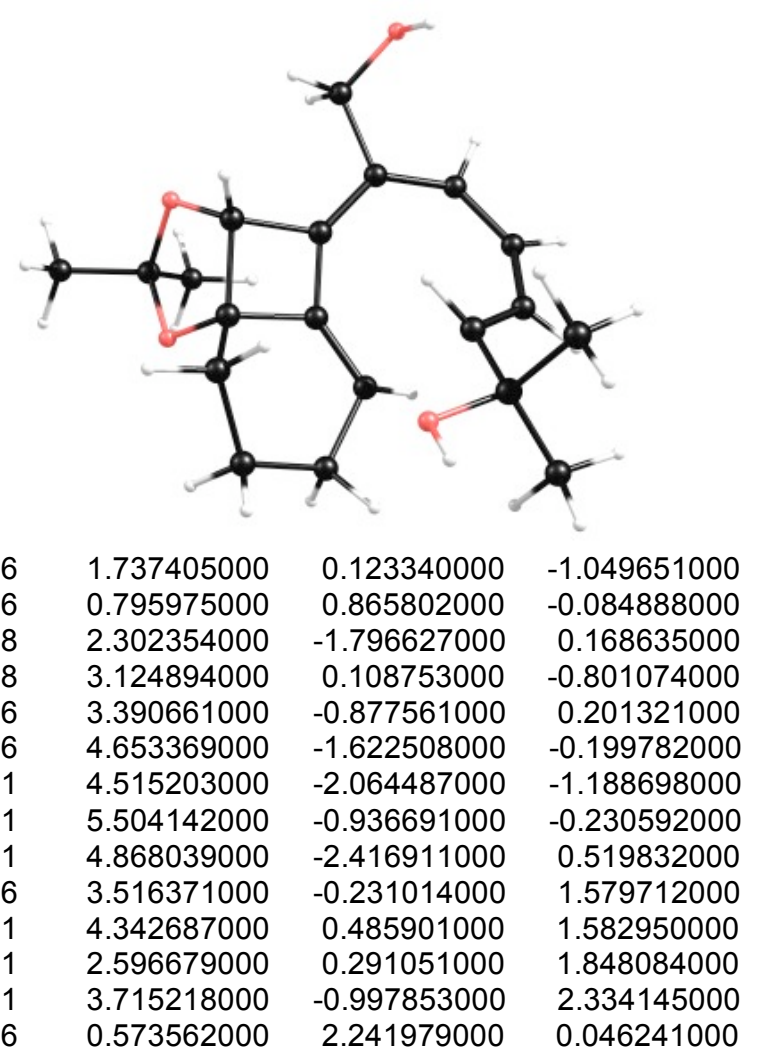




$\begin{array}{rrrr}6 & -2.354050000 & 0.388417000 & 0.057208000 \\ 6 & -2.516071000 & 1.412257000 & 0.991678000 \\ 6 & -0.431602000 & 2.897867000 & 0.768892000 \\ 6 & -1.678166000 & 2.497691000 & 1.264987000 \\ 6 & -3.537718000 & -0.455880000 & -0.381771000 \\ 6 & -4.425140000 & -0.934541000 & 0.771742000 \\ 1 & -3.842613000 & -1.470377000 & 1.524821000 \\ 1 & -4.944062000 & -0.105205000 & 1.260372000 \\ 1 & -5.195135000 & -1.613614000 & 0.388111000 \\ 6 & -4.370975000 & 0.361930000 & -1.385328000 \\ 1 & -3.750423000 & 0.667560000 & -2.232395000 \\ 1 & -5.204630000 & -0.240755000 & -1.769504000 \\ 1 & -4.788476000 & 1.258489000 & -0.919134000 \\ 8 & -2.975547000 & -1.581529000 & -1.064336000 \\ 1 & -3.705422000 & -2.063812000 & -1.470959000 \\ 6 & 1.640742000 & 3.128341000 & -0.589876000 \\ 1 & 2.519739000 & 3.174520000 & 0.063374000 \\ 1 & 1.985393000 & 2.660673000 & -1.525074000 \\ 8 & 1.252615000 & 4.470692000 & -0.798020000 \\ 1 & 0.459057000 & 4.457623000 & -1.346746000 \\ 1 & -0.201576000 & 3.946871000 & 0.955493000 \\ 1 & -2.115900000 & 3.219361000 & 1.955229000 \\ 1 & 1.580711000 & 0.332271000 & -2.114170000 \\ 1 & -1.638711000 & 0.545000000 & -0.743127000 \\ 6 & 0.284733000 & -0.341359000 & 0.423193000 \\ 6 & -0.787307000 & -0.861145000 & 1.142763000 \\ 6 & -0.961418000 & -2.366700000 & 1.106714000 \\ 6 & -0.265405000 & -3.136417000 & -0.052252000 \\ 6 & 0.356385000 & -2.258048000 & -1.155223000 \\ 6 & 1.173519000 & -1.194790000 & -0.461680000 \\ 1 & -0.590976000 & -2.755912000 & 2.066068000 \\ 1 & -2.031536000 & -2.588120000 & 1.095493000 \\ 1 & 0.537487000 & -3.749355000 & 0.368793000 \\ 1 & -0.984039000 & -3.825350000 & -0.506403000 \\ 1 & -0.435613000 & -1.779582000 & -1.740919000 \\ 1 & 0.970401000 & -2.869020000 & -1.826028000 \\ 1 & -3.405701000 & 1.365472000 & 1.619497000 \\ 1 & -1.163688000 & -0.364034000 & 2.025839000\end{array}$

\section{$16 a$}

$\mathrm{C} 2 \mathrm{OH} 28 \mathrm{O} 4$

C1

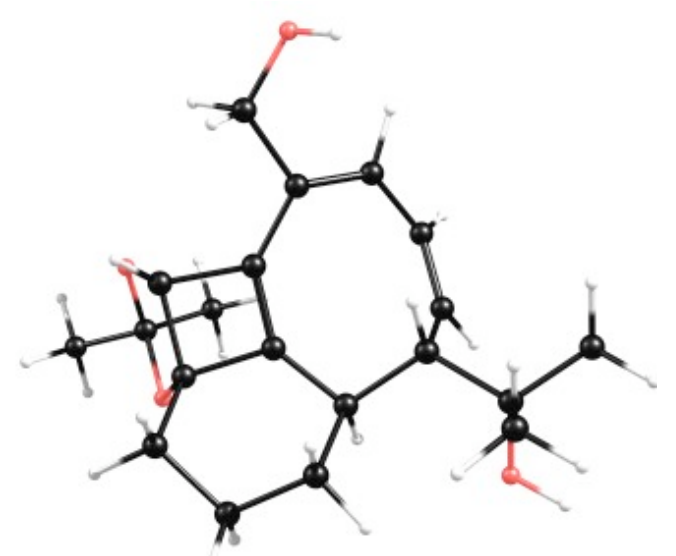

$\begin{array}{rrrr}6 & 2.141826000 & 0.174253000 & -0.967647000 \\ 6 & 0.810639000 & 0.774498000 & -0.492801000 \\ 8 & 2.363245000 & -1.695355000 & 0.435669000 \\ 8 & 3.280058000 & 0.326823000 & -0.152169000 \\ 6 & 3.224625000 & -0.650283000 & 0.894165000 \\ 6 & 4.618889000 & -1.230977000 & 1.063560000 \\ 1 & 5.318081000 & -0.450223000 & 1.374477000 \\ 1 & 4.610672000 & -2.017859000 & 1.822352000 \\ 1 & 4.955327000 & -1.653437000 & 0.114472000 \\ 6 & 2.697604000 & -0.034847000 & 2.188291000 \\ 1 & 3.359895000 & 0.773092000 & 2.512761000\end{array}$


0.414068000

$-0.795991000$

2.973476000

$-2.114865000$

2.171470000

$-0.420636000$

$-1.783309000$

0.043005000

0.804944000

0.210348000

$-0.418870000$

2.691638000

$-1.107559000$

1.962357000

1.465875000

0.512970000

1.566207000

1.145747000

3.092033000

$-1.381974000$

2.207652000

3.123030000

$-1.108496000$

1.102504000

2.661052000

0.699448000

4.428997000

$-2.396794000$

$-0.224196000$

4.422033000

3.779236000

$-0.475456$

2.427915000

0.363019000

2.430402000

0.284771000

$-0.443511000$

$-1.039647000$

$-1.073615000$

$-1.231843000$

$-2.171224000$

$-0.001193000$

$-3.080314000$

1.240958000

$-2.305961000$

1.534352000

$-1.205363000$

$-2.101129000$

$-2.777104000$

$-1.437835000$

$-1.701215000$

$-3.567832000$

0.214197000

$-3.881598000$

$-0.229041000$

$-1.852375000$

1.042883000

$-2.960819000$

0.357253000

$-2.164070000$

$-1.603064000$

$-0.425124000$

$-1.377014000$

$-1.655793000$

0.551913000

2.552236000

$-2.007970000$

$-0.198444000$

0.071260000

$-1.005482000$

$-1.153490000$

$-1.613318000$

$-0.608579000$

$-0.735324000$

$-1.977239000$

$-0.193573000$

$-1.866172000$

$-2.593614000$

$-1.717872000$

2.383299000

1.032800000

0.235086000

$-4.136240000 \quad-0.773377000$

$-1.162226000$

$-4.100376000$

0.099930000

$-1.821906000$

$-3.584467000$

$-1.583595000$

$-1.634818000$

$-1.088691000$

0.810622000

0.669109000

$-4.514974000$

1.599561000

0.240901000

0.757772000

$-5.562177000$

0.345985000

1.853374000

$-4.277947000$

0.887018000

$-1.573379000$

1.086583000

$-4.554157000$

$-1.828829000$

1.200085000

$-2.008480000$

0.718630000

$-0.648635000$

\section{$\mathrm{TS}^{2}(16 \mathrm{a} \rightarrow 22 \mathrm{a})$}

$\mathrm{C} 2 \mathrm{OH} 28 \mathrm{O} 4$

C1

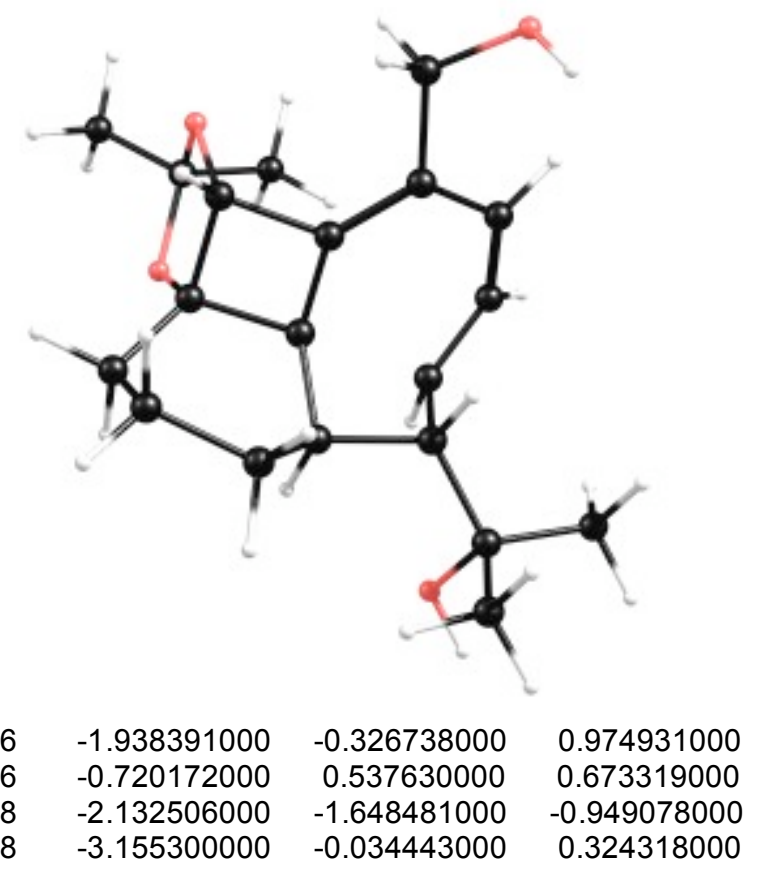




$\begin{array}{rrrr}6 & -3.105938000 & -0.601395000 & -0.990761000 \\ 6 & -4.460613000 & -1.224698000 & -1.280817000 \\ 1 & -5.238150000 & -0.456290000 & -1.280860000 \\ 1 & -4.449091000 & -1.712720000 & -2.258836000 \\ 1 & -4.692885000 & -1.966906000 & -0.514291000 \\ 6 & -2.716035000 & 0.449388000 & -2.025012000 \\ 1 & -3.456425000 & 1.254582000 & -2.038502000 \\ 1 & -1.736899000 & 0.876260000 & -1.799723000 \\ 1 & -2.676980000 & -0.006491000 & -3.018664000 \\ 6 & -0.586416000 & 1.916598000 & 0.664034000 \\ 6 & 2.153457000 & 0.088199000 & -0.075585000 \\ 6 & 1.257238000 & 0.540502000 & -1.228553000 \\ 6 & 0.151416000 & 2.562218000 & -0.355209000 \\ 6 & 0.809182000 & 1.872797000 & -1.360165000 \\ 6 & -1.556032000 & 2.753274000 & 1.486188000 \\ 1 & -2.543100000 & 2.737268000 & 1.010827000 \\ 1 & -1.681682000 & 2.300634000 & 2.481735000 \\ 8 & -1.192496000 & 4.115742000 & 1.586130000 \\ 1 & -0.326970000 & 4.151042000 & 2.010423000 \\ 1 & -0.050664000 & 3.623885000 & -0.480198000 \\ 1 & 0.853086000 & 2.346122000 & -2.341699000 \\ 1 & -2.146298000 & -0.533594000 & 2.030248000 \\ 6 & -0.032308000 & -0.518930000 & -0.002030000 \\ 6 & 1.318150000 & -1.132995000 & 0.296613000 \\ 6 & 1.369063000 & -1.826067000 & 1.663583000 \\ 6 & 0.042691000 & -2.564887000 & 1.997354000 \\ 6 & -0.899147000 & -2.775098000 & 0.800192000 \\ 6 & -1.262745000 & -1.452980000 & 0.143775000 \\ 1 & 2.201434000 & -2.538301000 & 1.680538000 \\ 1 & 1.577212000 & -1.083757000 & 2.443848000 \\ 1 & 0.262558000 & -3.536377000 & 2.452235000 \\ 1 & -0.500855000 & -2.000372000 & 2.764459000 \\ 1 & -1.814064000 & -3.287019000 & 1.120021000 \\ 1 & -0.426822000 & -3.416197000 & 0.044774000 \\ 1 & 1.476142000 & 0.002002000 & -2.151607000 \\ 1 & 1.504127000 & -1.883546000 & -0.482638000 \\ 6 & 3.632081000 & -0.112675000 & -0.461740000 \\ 6 & 4.455678000 & -0.580793000 & 0.742890000 \\ 1 & 4.412404000 & 0.141815000 & 1.564435000 \\ 1 & 4.098161000 & -1.545558000 & 1.108714000 \\ 1 & 5.509545000 & -0.696044000 & 0.461177000 \\ 6 & 4.226684000 & 1.179608000 & -1.029426000 \\ 1 & 4.182224000 & 1.993112000 & -0.298218000 \\ 1 & 5.280281000 & 1.030396000 & -1.296830000 \\ 1 & 3.685720000 & 1.494663000 & -1.924830000 \\ 8 & 3.633020000 & -1.129150000 & -1.468433000 \\ 1 & 4.544016000 & -1.247506000 & -1.761279000 \\ 1 & 2.105180000 & 0.831801000 & 0.729753000\end{array}$

22a

$\mathrm{C} 20 \mathrm{H} 28 \mathrm{O} 4$

C1

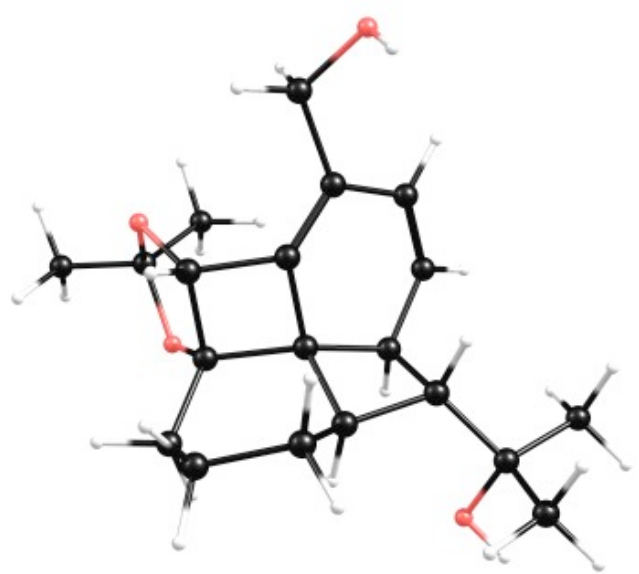

6

$\begin{array}{lll}-1.793068000 & -0.479290000 & 0.984890000\end{array}$ 


\begin{tabular}{|c|c|c|}
\hline-0.735705000 & 0.576024000 & 0.719785000 \\
\hline-1.900964000 & -1.545898000 & -1.101636000 \\
\hline-3.072187000 & -0.279989000 & 0.423195000 \\
\hline-3.060623000 & -0.723733000 & -0.937945000 \\
\hline-4.296240000 & -1.582936000 & -1.157002000 \\
\hline-5.202243000 & -0.987386000 & -1.016102000 \\
\hline-4.298171000 & -1.990848000 & -2.171200000 \\
\hline-4.299446000 & -2.407938000 & -0.441298000 \\
\hline-2.995893000 & 0.455658000 & -1.901296000 \\
\hline-3.858604000 & 1.111280000 & -1.751879000 \\
\hline-2.081508000 & 1.031390000 & -1.746827000 \\
\hline-3.008496000 & 0.092392000 & -2.932912000 \\
\hline-0.859696000 & 1.907977000 & 0.543194000 \\
\hline 2.234384000 & 0.229064000 & 0.017141000 \\
\hline 1.101868000 & 0.442571000 & -1.054996000 \\
\hline-0.033584000 & 2.572494000 & -0.463202000 \\
\hline 0.758282000 & 1.871965000 & -1.299371000 \\
\hline-1.989640000 & 2.705491000 & 1.146101000 \\
\hline-2.697370000 & 3.010562000 & 0.366506000 \\
\hline-2.548726000 & 2.092499000 & 1.865603000 \\
\hline-1.529839000 & 3.917980000 & 1.728938000 \\
\hline-0.867897000 & 3.679058000 & 2.388438000 \\
\hline-0.195910000 & 3.636719000 & -0.614009000 \\
\hline 1.215915000 & 2.371149000 & -2.151049000 \\
\hline-1.915226000 & -0.838304000 & 2.011535000 \\
\hline 0.154568000 & -0.352696000 & -0.056190000 \\
\hline 1.429184000 & -0.975315000 & 0.532299000 \\
\hline 1.283272000 & -1.550136000 & 1.923936000 \\
\hline 0.213912000 & -2.686122000 & 1.854129000 \\
\hline-0.580209000 & -2.760901000 & 0.526507000 \\
\hline-1.030656000 & -1.403509000 & -0.006774000 \\
\hline 2.228444000 & -1.946682000 & 2.310234000 \\
\hline 0.962773000 & -0.763021000 & 2.618314000 \\
\hline 0.697648000 & -3.657252000 & 2.003995000 \\
\hline-0.489562000 & -2.577762000 & 2.687019000 \\
\hline-1.471784000 & -3.381232000 & 0.670825000 \\
\hline 0.016362000 & -3.256517000 & -0.248571000 \\
\hline 1.348962000 & -0.107467000 & -1.967929000 \\
\hline 1.695621000 & -1.793691000 & -0.151006000 \\
\hline 3.675451000 & 0.021607000 & -0.456582000 \\
\hline 4.577919000 & -0.341571000 & 0.727587000 \\
\hline 4.583271000 & 0.449684000 & 1.484190000 \\
\hline 4.240358000 & -1.269495000 & 1.196148000 \\
\hline 5.612606000 & -0.486759000 & 0.393218000 \\
\hline 4.202133000 & 1.272885000 & -1.165759000 \\
\hline 4.177399000 & 2.147643000 & -0.508210000 \\
\hline 5.241625000 & 1.125768000 & -1.484703000 \\
\hline 3.603870000 & 1.491245000 & -2.054109000 \\
\hline 3.632985000 & -1.069905000 & -1.380549000 \\
\hline 4.530771000 & -1.213684000 & -1.701478000 \\
\hline 2.205395000 & 1.063986000 & 0.726946000 \\
\hline
\end{tabular}

IId.

$11 a$

$\mathrm{C} 21 \mathrm{H} 30 \mathrm{O} 4$

C1 


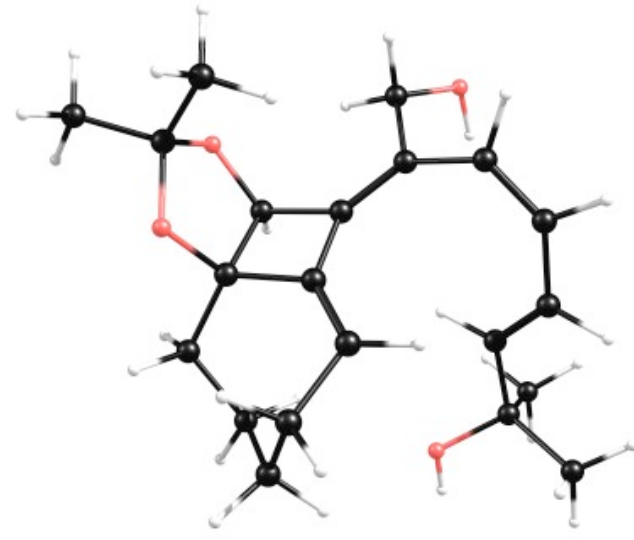

\begin{tabular}{|c|c|c|}
\hline-1.661297000 & -1.153253000 & -0.276530000 \\
\hline-1.375184000 & -2.499191000 & -0.946866000 \\
\hline-0.572011000 & -0.528311000 & 0.601671000 \\
\hline 0.100015000 & -2.915684000 & -0.926467000 \\
\hline 0.252914000 & -1.145359000 & 1.464055000 \\
\hline 0.119142000 & -2.627656000 & 1.663192000 \\
\hline-1.748795000 & -2.464254000 & -1.978084000 \\
\hline-1.983484000 & -3.251747000 & -0.430478000 \\
\hline 0.729585000 & -2.076865000 & -1.241708000 \\
\hline 0.258753000 & -3.705334000 & -1.67098500 \\
\hline 0.673076000 & -2.956501000 & 2.548593000 \\
\hline-0.935660000 & -2.865993000 & 1.848133000 \\
\hline 1.050186000 & -0.600815000 & 1.958794000 \\
\hline-1.698209000 & 0.187465000 & -1.065965000 \\
\hline-0.723255000 & 0.777988000 & -0.035707000 \\
\hline-2.962863000 & -1.208686000 & \\
\hline-3.037717000 & 0.617455000 & -1.069845000 \\
\hline-3.717048000 & -0.038571000 & 0.004869000 \\
\hline-1.331900000 & 0.173336000 & -2.098369000 \\
\hline-0.340078000 & 2481000 & 0.17 \\
\hline 218000 & 4000 & 1.26 \\
\hline 326000 & 166000 & 1.70 \\
\hline 2.749228000 & 834000 & 1.17 \\
\hline 2.718607000 & 2522000 & 0.06022000 \\
\hline 3.663645000 & 1.280694000 & 1.76682000 \\
\hline 1.828032000 & 0.520526000 & -0.56242900 \\
\hline-0.972760000 & 3.162784000 & -0.653266000 \\
\hline-1.500172000 & 2.734567000 & -1.516208000 \\
\hline-1.730085000 & 3.681493000 & -0.05066000 \\
\hline-0.051869000 & 4.169641000 & -1.038493000 \\
\hline 0.690611000 & 3.728678000 & -1.467277000 \\
\hline-3.809109000 & 0.867919000 & 1.229702000 \\
\hline-4.374825000 & 1.772551000 & 0.988360000 \\
\hline-4.318485000 & 0.342321000 & 2.042363000 \\
\hline-2.813389000 & 1.154083000 & 1.572336000 \\
\hline-5.086336000 & -0.463420000 & -0.501645000 \\
\hline-5.676107000 & 0.413108000 & -0.782771000 \\
\hline-4.966940000 & -1.108107000 & -1.374893000 \\
\hline-5.622402000 & -1.012686000 & 0.276649000 \\
\hline 3.870312000 & 385000 & $-0.500773 c$ \\
\hline 4.961230000 & -0.5 & 0.520948000 \\
\hline 4.539491000 & -1.119715000 & 1.371771000 \\
\hline 5.7280 & -1.210013000 & 0.057889000 \\
\hline 5.461911000 & 0.322796000 & 0.886788000 \\
\hline 4.459515000 & 0.504323000 & -1.701672000 \\
\hline 3.683192000 & 0.696531000 & -2.447823000 \\
\hline 4.890741000 & 1.460891000 & -1.392957000 \\
\hline 5.252898000 & -0.086513000 & -2.177656000 \\
\hline 3.274997000 & -1.475173000 & -0.96578400 \\
\hline 3.954542000 & -1.960524000 & -1.449484000 \\
\hline 0.066295000 & 3.410868000 & 1.776820000 \\
\hline 2.079405000 & 2.757667000 & 2.569965000 \\
\hline 0.603685000 & -3.431803000 & 0.432477000 \\
\hline 1.698721000 & -3.429789000 & 0.409078000 \\
\hline
\end{tabular}


$\mathrm{TS}^{1}(11 \mathrm{a} \rightarrow 17 \mathrm{a})$

$\mathrm{C} 21 \mathrm{H} 30 \mathrm{O} 4$

C1

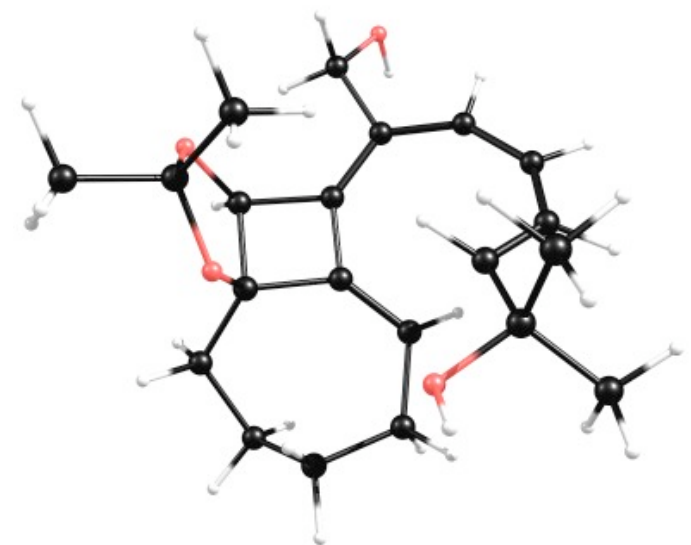

$\begin{array}{lrrr}6 & -1.026027000 & -1.480178000 & -0.556122000 \\ 6 & -1.067995000 & -2.729183000 & -1.441145000 \\ 6 & -0.218286000 & -0.246999000 & -0.980195000 \\ 6 & 0.150652000 & -2.912912000 & -2.351267000 \\ 6 & 1.082698000 & -0.047213000 & -1.453301000 \\ 6 & 1.976131000 & -1.173122000 & -1.924570000 \\ 1 & -1.980852000 & -2.719289000 & -2.049966000 \\ 1 & -1.152338000 & -3.586920000 & -0.765835000 \\ 1 & 0.048637000 & -2.275142000 & -3.239696000 \\ 1 & 0.146552000 & -3.943983000 & -2.724174000 \\ 1 & 2.107660000 & -1.043652000 & -3.010326000 \\ 1 & 2.971064000 & -1.056635000 & -1.487786000 \\ 1 & 1.227946000 & 0.883117000 & -1.989132000 \\ 6 & -2.244910000 & -0.528234000 & -0.454421000 \\ 6 & -1.271789000 & 0.631044000 & -0.671380000 \\ 8 & -0.815485000 & -1.850916000 & 0.811049000 \\ 8 & -2.823898000 & -0.723646000 & 0.812653000 \\ 6 & -1.863636000 & -1.364847000 & 1.653663000 \\ 1 & -3.025530000 & -0.646584000 & -1.213746000 \\ 6 & -1.492273000 & 2.006540000 & -0.536475000 \\ 6 & -0.576339000 & 3.068273000 & -0.573700000 \\ 6 & 0.819979000 & 3.195912000 & -0.533858000 \\ 6 & 1.867503000 & 2.342196000 & -0.186660000 \\ 6 & 1.817207000 & 1.045559000 & 0.337789000 \\ 1 & 2.857825000 & 2.744528000 & -0.396651000 \\ 6 & -2.955847000 & 2.409828000 & -0.319002000 \\ 1 & -3.619854000 & 1.628234000 & -0.712356000 \\ 1 & -3.161607000 & 2.477842000 & 0.755070000 \\ 8 & -3.300099000 & 3.676051000 & -0.849876000 \\ 1 & -3.153339000 & 3.634741000 & -1.802524000 \\ 6 & -1.295635000 & -0.372892000 & 2.662319000 \\ 1 & -2.081011000 & -0.035097000 & 3.344610000 \\ 1 & -0.500661000 & -0.845889000 & 3.245522000 \\ 1 & -0.887819000 & 0.497456000 & 2.144912000 \\ 6 & -2.545008000 & -2.546429000 & 2.330515000 \\ 1 & -3.375074000 & -2.201984000 & 2.953606000 \\ 1 & -2.932817000 & -3.228866000 & 1.570850000 \\ 1 & -1.831411000 & -3.084258000 & 2.960465000 \\ 1 & -1.083792000 & 4.029831000 & -0.649612000 \\ 1 & 1.155297000 & 4.200550000 & -0.793482000 \\ 6 & 1.487731000 & -2.598040000 & -1.661386000 \\ 1 & 2.262808000 & -3.283794000 & -2.024019000 \\ 1 & 1.411722000 & -2.761321000 & -0.580226000 \\ 6 & 3.059206000 & 0.426913000 & 0.963658000 \\ 6 & 4.361441000 & 0.722234000 & 0.209248000 \\ 1 & 4.648091000 & 1.775306000 & 0.280571000 \\ 1 & 5.177396000 & 0.136902000 & 0.648092000 \\ & 4.284929000 & 0.457446000 & -0.848114000\end{array}$




$\begin{array}{rrrr}6 & 3.169309000 & 0.940912000 & 2.410867000 \\ 1 & 2.266612000 & 0.686001000 & 2.972514000 \\ 1 & 4.029533000 & 0.479308000 & 2.913658000 \\ 1 & 3.303403000 & 2.025845000 & 2.440738000 \\ 8 & 2.819864000 & -0.981679000 & 0.992980000 \\ 1 & 3.522794000 & -1.380706000 & 1.519607000 \\ 1 & 0.916115000 & 0.742360000 & 0.855512000\end{array}$

$17 a$

$\mathrm{C} 21 \mathrm{H} 30 \mathrm{O} 4$

C1

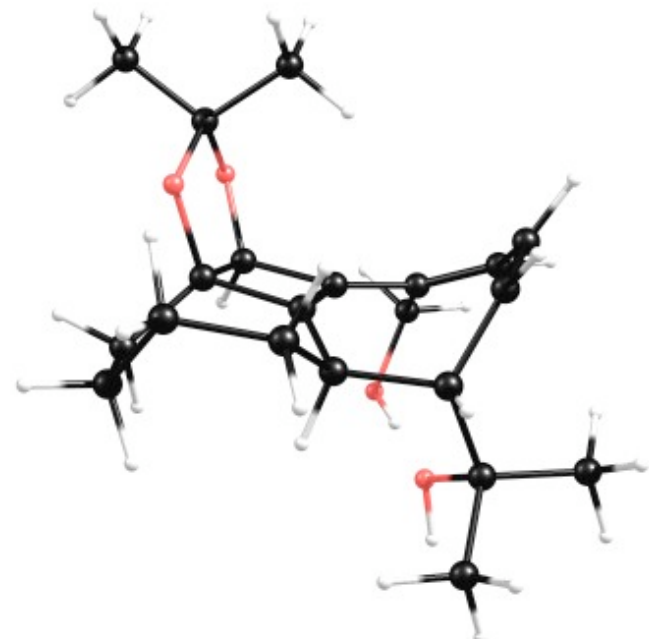

$\begin{array}{rrrr}6 & 1.638888000 & 0.577493000 & -0.703056000 \\ 6 & 1.756615000 & 1.672290000 & -1.749408000 \\ 6 & 0.253807000 & 0.360341000 & -0.100864000 \\ 6 & 1.300505000 & 3.073058000 & -1.245704000 \\ 6 & -0.760393000 & 1.414652000 & 0.185913000 \\ 6 & -0.159128000 & 2.653347000 & 0.882473000 \\ 1 & 1.159503000 & 1.380239000 & -2.621399000 \\ 1 & 2.800556000 & 1.708665000 & -2.077248000 \\ 1 & 0.356184000 & 3.351774000 & -1.731100000 \\ 1 & 2.029977000 & 3.815768000 & -1.586292000 \\ 1 & -0.931925000 & 3.433706000 & 0.851181000 \\ 1 & 0.007227000 & 2.429749000 & 1.943911000 \\ 1 & -1.073351000 & 1.753911000 & -0.815099000 \\ 6 & 1.613429000 & -0.938324000 & -1.036155000 \\ 6 & 0.280570000 & -0.980626000 & -0.291420000 \\ 8 & 2.724105000 & 0.683645000 & 0.222085000 \\ 8 & 2.756709000 & -1.508969000 & -0.444909000 \\ 6 & 3.226415000 & -0.613102000 & 0.560419000 \\ 1 & 1.569307000 & -1.237079000 & -2.087620000 \\ 6 & -0.576347000 & -2.121482000 & -0.022037000 \\ 6 & -1.326548000 & -2.264347000 & 1.092065000 \\ 6 & -1.465493000 & -1.301382000 & 2.174871000 \\ 6 & -1.733127000 & 0.014690000 & 2.099372000 \\ 6 & -2.038599000 & 0.923172000 & 0.919158000 \\ 1 & -1.785870000 & 0.527717000 & 3.059263000 \\ 6 & -0.609102000 & -3.160545000 & -1.112634000 \\ 1 & 0.395234000 & -3.551398000 & -1.318550000 \\ 1 & -1.239088000 & -4.009827000 & -0.804616000 \\ 8 & -1.077785000 & -2.569565000 & -2.315665000 \\ 1 & -1.700231000 & -1.880326000 & -2.037631000 \\ 6 & 2.741925000 & -1.034483000 & 1.946258000 \\ 1 & 3.117393000 & -2.034178000 & 2.183459000 \\ 1 & 3.114806000 & -0.333200000 & 2.698900000 \\ 1 & 1.652266000 & -1.046340000 & 1.991182000 \\ 6 & 4.743924000 & -0.567318000 & 0.476806000 \\ 1 & 5.164726000 & -1.551074000 & 0.701680000 \\ 1 & 5.044507000 & -0.273684000 & -0.531097000 \\ 1 & 5.140771000 & 0.156495000 & 1.193578000 \\ 1 & -1.840171000 & -3.217519000 & 1.230957000\end{array}$


C1

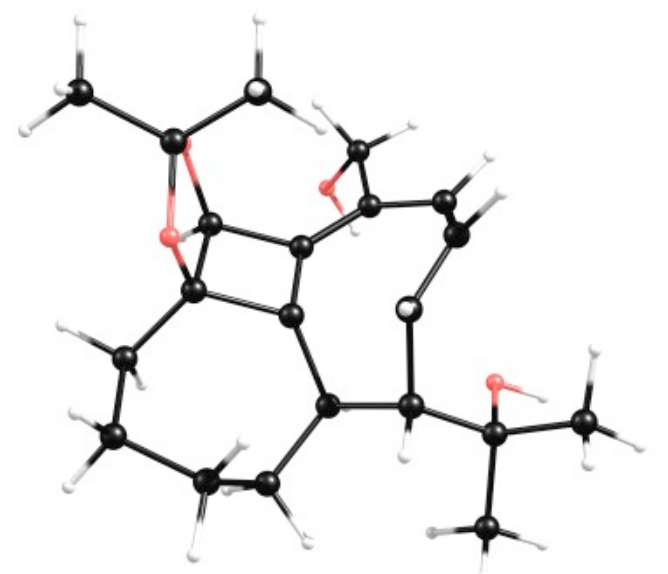

$\begin{array}{rrrr}6 & 1.465456000 & 1.009550000 & -0.430731000 \\ 6 & 1.602992000 & 2.256831000 & -1.282103000 \\ 6 & 0.061303000 & 0.349889000 & -0.268081000 \\ 6 & 0.992609000 & 3.513377000 & -0.660690000 \\ 6 & -1.297239000 & 0.929948000 & -0.697323000 \\ 6 & -1.304298000 & 2.426190000 & -1.069889000 \\ 1 & 1.134740000 & 2.054659000 & -2.253506000 \\ 1 & 2.669645000 & 2.417243000 & -1.479578000 \\ 1 & 1.009658000 & 4.311475000 & -1.414078000 \\ 1 & 1.623704000 & 3.853019000 & 0.167821000 \\ 1 & -0.995010000 & 2.563303000 & -2.113637000 \\ 1 & -2.347723000 & 2.759708000 & -1.031994000 \\ 1 & -1.666614000 & 0.347542000 & -1.549590000 \\ 6 & 1.952087000 & -0.341419000 & -1.016277000 \\ 6 & 0.589448000 & -0.905237000 & -0.660375000 \\ 8 & 2.216891000 & 1.147177000 & 0.764215000 \\ 8 & 3.083576000 & -0.721675000 & -0.266125000 \\ 6 & 3.080104000 & 0.017803000 & 0.955625000 \\ 1 & 2.212779000 & -0.352003000 & -2.078688000 \\ 6 & 0.150284000 & -2.200529000 & -0.428059000 \\ 6 & -0.666216000 & -2.458980000 & 0.689118000 \\ 6 & -0.991718000 & -1.498477000 & 1.639756000 \\ 6 & -1.099706000 & -0.102906000 & 1.454129000 \\ 6 & -2.121190000 & 0.622935000 & 0.553717000 \\ 1 & -0.913960000 & 0.455303000 & 2.375462000 \\ 6 & 0.847194000 & -3.346993000 & -1.125378000 \\ 1 & 1.909101000 & -3.356636000 & -0.854326000 \\ 1 & 0.412716000 & -4.302802000 & -0.790612000 \\ 8 & 0.820906000 & -3.231431000 & -2.538990000 \\ 1 & -0.097942000 & -3.077885000 & -2.789064000 \\ 6 & 2.570080000 & -0.840855000 & 2.106140000 \\ 1 & 3.234229000 & -1.697300000 & 2.255359000\end{array}$


$-0.313224000$

1.318669000

4.816504000

1.131501000

0.348451000

4.523617000

1.138833000

2.103635000

$-0.770599000$

$-3.505992000$

0.974984000

$-0.998034000$

$-1.854380000$

$-0.444814000$

3.313811000

672814000

$-0.914410000$

4.296975000

$-0.413163000$

2.874203000

$-0.154698000$

$-0.034291000$

$-3.494016000$

$-0.047902000$

0.850567000

$-4.150480000$

$-0.446546000$

0.325057000

$-4.279642000$

0.419509000

650600000

$-5.145655000$

$-0.870998000$

2.308496000

$-3.548210000$

$-1.193921000$

(1971000

$-4.421807000$

0.908332000

2.170455000

$-4.005361000$

1.156613000

$-0.439641000$

$-5.395515000$

0.430838000

$-1.419403000$

$-4.600423000$

1.836414000

$-0.603983000$

$-3.266186000$

$-1.205268000$

.113433000

$-4.114666000$

$-1.651448000$

$-0.478667000$

$-2.349513000$

1.566170000

585128000

1.067688000

\section{3a}

C21H30O4

C1

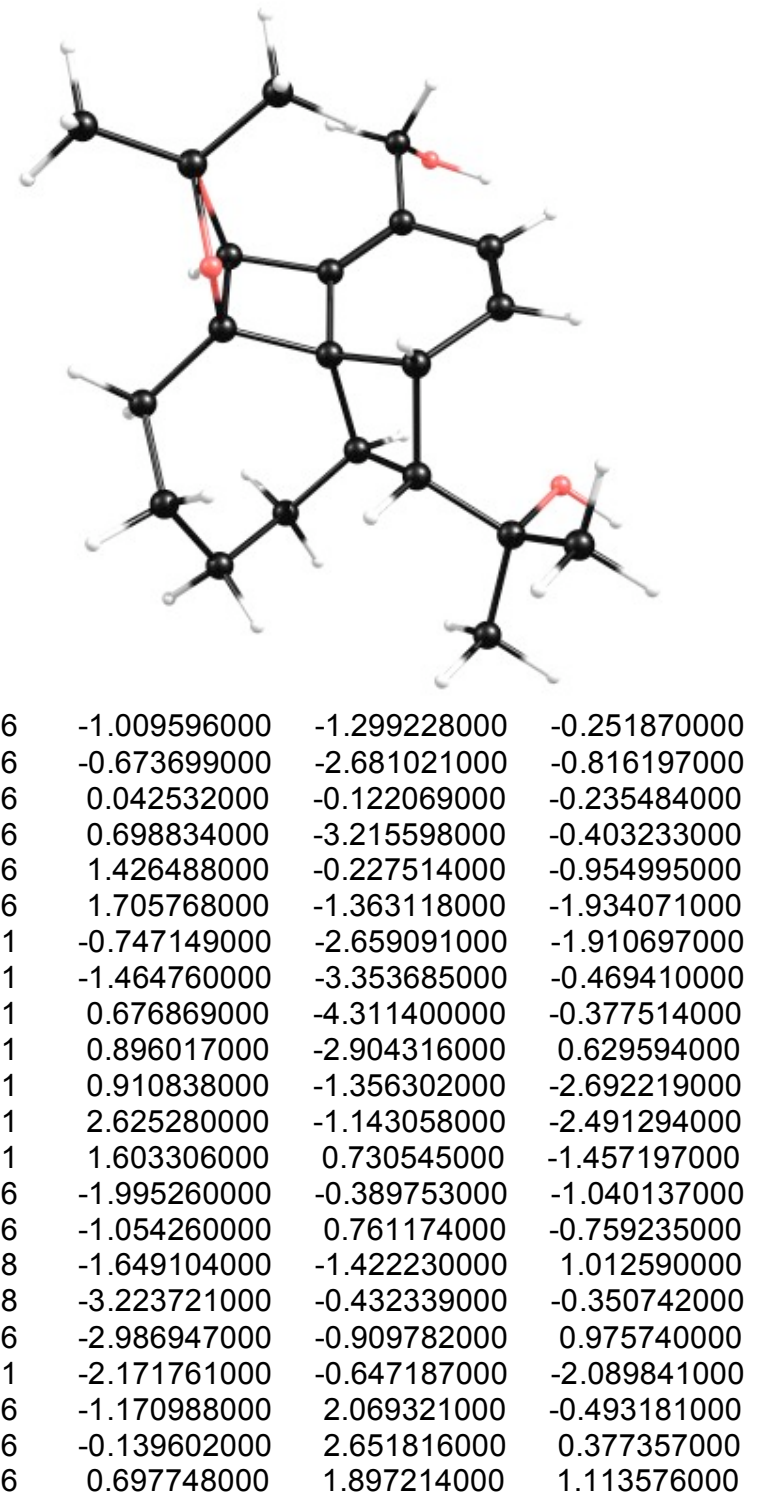




$\begin{array}{rrrr}6 & 0.787719000 & 0.395023000 & 1.032671000 \\ 6 & 2.113333000 & -0.180005000 & 0.434413000 \\ 1 & 0.504135000 & -0.048903000 & 1.996269000 \\ 6 & -2.352290000 & 2.911314000 & -0.900494000 \\ 1 & -3.147469000 & 2.274595000 & -1.294462000 \\ 1 & -2.756745000 & 3.435953000 & -0.018406000 \\ 8 & -2.036481000 & 3.835703000 & -1.935174000 \\ 1 & -1.298015000 & 4.374817000 & -1.629518000 \\ 6 & -3.108674000 & 0.220681000 & 1.989946000 \\ 1 & -4.114045000 & 0.649777000 & 1.956131000 \\ 1 & -2.924394000 & -0.159755000 & 2.998860000 \\ 1 & -2.379206000 & 1.002088000 & 1.770217000 \\ 6 & -3.973112000 & -2.038323000 & 1.246256000 \\ 1 & -4.999224000 & -1.662189000 & 1.209343000 \\ 1 & -3.859959000 & -2.818556000 & 0.490059000 \\ 1 & -3.792635000 & -2.470493000 & 2.234110000 \\ 1 & -0.139119000 & 3.731100000 & 0.527725000 \\ 1 & 1.341632000 & 2.391265000 & 1.835763000 \\ 6 & 1.836883000 & -2.771514000 & -1.339332000 \\ 1 & 1.901112000 & -3.476399000 & -2.178523000 \\ 1 & 2.793481000 & -2.860109000 & -0.809123000 \\ 6 & 3.468730000 & 0.509155000 & 0.622388000 \\ 6 & 3.827264000 & 0.581567000 & 2.112463000 \\ 1 & 3.913047000 & -0.418685000 & 2.549799000 \\ 1 & 4.791602000 & 1.085764000 & 2.251112000 \\ 1 & 3.072477000 & 1.137047000 & 2.674543000 \\ 6 & 4.563824000 & -0.250809000 & -0.137173000 \\ 1 & 4.355478000 & -0.253769000 & -1.209909000 \\ 1 & 5.537444000 & 0.231455000 & 0.016330000 \\ 1 & 4.653490000 & -1.286213000 & 0.206667000 \\ 8 & 3.352851000 & 1.824345000 & 0.080158000 \\ 1 & 4.214306000 & 2.249482000 & 0.168452000 \\ 1 & 2.227430000 & -1.198799000 & 0.822032000\end{array}$

Ile.

$12 a$

$\mathrm{C} 21 \mathrm{H} 30 \mathrm{O} 4$

C1

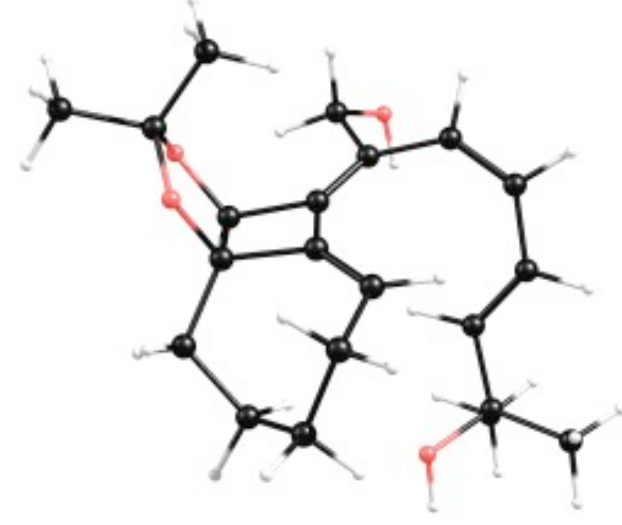

$\begin{array}{rrrr}6 & -1.661297000 & -1.153253000 & -0.276530000 \\ 6 & -1.375184000 & -2.499191000 & -0.946866000 \\ 6 & -0.572011000 & -0.528311000 & 0.601671000 \\ 6 & 0.100015000 & -2.915684000 & -0.926467000 \\ 6 & 0.252914000 & -1.145359000 & 1.464055000 \\ 6 & 0.119142000 & -2.627656000 & 1.663192000 \\ 1 & -1.748795000 & -2.464254000 & -1.978084000 \\ 1 & -1.983484000 & -3.251747000 & -0.430478000 \\ 1 & 0.729585000 & -2.076865000 & -1.241708000 \\ 1 & 0.258753000 & -3.705334000 & -1.670985000 \\ 1 & 0.673076000 & -2.956501000 & 2.548593000 \\ 1 & -0.935660000 & -2.865993000 & 1.848133000 \\ 1 & 1.050186000 & -0.600815000 & 1.958794000\end{array}$




\begin{tabular}{rrrr}
6 & -1.698209000 & 0.187465000 & -1.065965000 \\
6 & -0.723255000 & 0.777988000 & -0.035707000 \\
8 & -2.962863000 & -1.208686000 & 0.308996000 \\
8 & -3.037717000 & 0.617455000 & -1.069845000 \\
6 & -3.717048000 & -0.038571000 & 0.004869000 \\
1 & -1.331900000 & 0.173336000 & -2.098369000 \\
6 & -0.340078000 & 2.062481000 & 0.174856000 \\
6 & 0.500218000 & 2.548554000 & 1.266004000 \\
6 & 1.734826000 & 2.197166000 & 1.701635000 \\
6 & 2.749228000 & 1.293834000 & 1.175082000 \\
6 & 2.718607000 & 0.542522000 & 0.060220000 \\
1 & 3.663645000 & 1.280694000 & 1.766820000 \\
1 & 1.828032000 & 0.520526000 & -0.562429000 \\
6 & -0.972760000 & 3.162784000 & -0.653266000 \\
1 & -1.500172000 & 2.734567000 & -1.516208000 \\
1 & -1.730085000 & 3.681493000 & -0.050660000 \\
8 & -0.051869000 & 4.169641000 & -1.038493000 \\
1 & 0.690611000 & 3.728678000 & -1.467277000 \\
6 & -3.809109000 & 0.867919000 & 1.229702000 \\
1 & -4.374825000 & 1.772551000 & 0.988360000 \\
1 & -4.318485000 & 0.342321000 & 2.042363000 \\
1 & -2.813389000 & 1.154083000 & 1.572336000 \\
6 & -5.086336000 & -0.463420000 & -0.501645000 \\
1 & -5.676107000 & 0.413108000 & -0.782771000 \\
1 & -4.966940000 & -1.108107000 & -1.374893000 \\
1 & -5.622402000 & -1.012686000 & 0.276649000 \\
6 & 3.870312000 & -0.255385000 & -0.500773000 \\
6 & 4.961230000 & -0.578663000 & 0.520948000 \\
1 & 4.539491000 & -1.119715000 & 1.371771000 \\
1 & 5.728092000 & -1.210013000 & 0.057889000 \\
1 & 5.461911000 & 0.322796000 & 0.886788000 \\
6 & 4.459515000 & 0.504323000 & -1.701672000 \\
1 & 3.683192000 & 0.696531000 & -2.447823000 \\
1 & 4.890741000 & 1.460891000 & -1.392957000 \\
1 & 5.252898000 & -0.086513000 & -2.177656000 \\
8 & 3.274997000 & -1.475173000 & -0.965784000 \\
1 & 3.954542000 & -1.960524000 & -1.449484000 \\
1 & 0.066295000 & 3.410868000 & 1.776820000 \\
1 & 2.079405000 & 2.757667000 & 2.569965000 \\
6 & 0.603685000 & -3.431803000 & 0.432477000 \\
1 & 1.698721000 & -3.429789000 & 0.409078000 \\
1 & 0.286251000 & -4.473589000 & 0.573493000 \\
TS ${ }^{1} \mathbf{( 1 2 a - 1 8 a )}$ & & \\
& & & \\
\hline
\end{tabular}

\section{$\mathrm{C} 21 \mathrm{H} 30 \mathrm{O} 4$}

C1

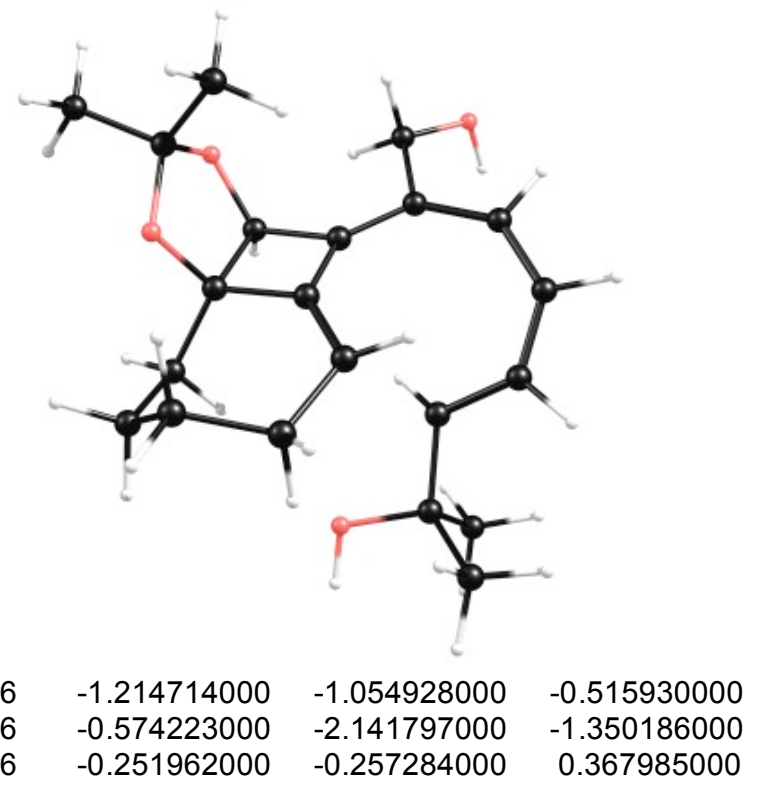




\begin{tabular}{|c|c|c|c|}
\hline 5 & -0.271913000 & -3.397195000 & -0.505360000 \\
\hline 5 & 0.828178000 & -0.686621000 & 1.148843000 \\
\hline & 1.147948000 & -2.162488000 & 1.315100000 \\
\hline & 0.358727000 & -1.740315000 & -1.760733000 \\
\hline 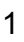 & -1.223756000 & -2.406656000 & -2.193193000 \\
\hline 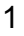 & 0.570128000 & -3.931321000 & -0.963068000 \\
\hline 1 & -1.132895000 & -4.072883000 & -0.556701000 \\
\hline | & 2.014137000 & -2.408540000 & 0.691610000 \\
\hline 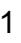 & 1.459926000 & -2.322770000 & 2.355100000 \\
\hline 1 & 1.103851000 & -0.054212000 & 1.981353000 \\
\hline 6 & -1.702517000 & 0.306458000 & -1.074268000 \\
\hline 6 & -0.729780000 & 0.979045000 & -0.100751000 \\
\hline 8 & -2.379210000 & -1.572760000 & 0.131164000 \\
\hline 8 & -3.091247000 & 0.371082000 & -0.840991000 \\
\hline 6 & -3.417433000 & -0.595475000 & 0.158504000 \\
\hline 1 & -1.524027000 & 0.516509000 & -2.135143000 \\
\hline 6 & -0.483266000 & 2.349827000 & 0.079548000 \\
\hline 6 & 0.532910000 & 2.986037000 & 0.802338000 \\
\hline 6 & 1.796394000 & 2.591664000 & 1.268115000 \\
\hline 6 & 2.622838000 & 1.511340000 & 0.961943000 \\
\hline 6 & 2.395955000 & 0.488593000 & 0.035082000 \\
\hline 1 & 3.536707000 & 1.452006000 & 1.552401000 \\
\hline 1 & 1.661563000 & 0.678303000 & -0.740206000 \\
\hline 6 & -1.556107000 & 3.263796000 & -0.512217000 \\
\hline 1 & -1.905942000 & 2.835862000 & -1.464022000 \\
\hline 1 & -2.431782000 & 3.281813000 & 0.146630000 \\
\hline 8 & -1.171348000 & 4.614078000 & -0.667548000 \\
\hline 1 & -0.386502000 & 4.625687000 & -1.228800000 \\
\hline 6 & -3.513087000 & 0.055105000 & 1.537198000 \\
\hline 1 & -4.301769000 & 0.813229000 & 1.539367000 \\
\hline 1 & -3.753072000 & -0.700762000 & 2.290722000 \\
\hline 1 & -2.567915000 & 0.528530000 & 1.807335000 \\
\hline 6 & -4.717021000 & -1.270496000 & -0.248819000 \\
\hline 1 & -5.528914000 & -0.539100000 & -0.281804000 \\
\hline 1 & -4.599298000 & -1.717693000 & -1.238024000 \\
\hline 1 & -4.978056000 & -2.052930000 & 0.468538000 \\
\hline 6 & 3.549852000 & -0.361809000 & -0.468335000 \\
\hline 6 & 4.463340000 & -0.896080000 & 0.639402000 \\
\hline 1 & 3.897246000 & -1.458606000 & 1.384490000 \\
\hline 1 & 5.219929000 & -1.561116000 & 0.207155000 \\
\hline 1 & 4.999235000 & -0.089070000 & 1.147103000 \\
\hline 6 & 4.367801000 & 0.475072000 & -1.468631000 \\
\hline 1 & 5.170477000 & -0.133044000 & -1.906266000 \\
\hline 1 & 3.725983000 & 0.830181000 & -2.279647000 \\
\hline 1 & 4.825653000 & 1.341186000 & -0.983084000 \\
\hline 8 & 2.944440000 & -1.450645000 & -1.176791000 \\
\hline 1 & 3.654542000 & -1.950088000 & -1.597701000 \\
\hline 1 & 0.302921000 & 4.031299000 & 1.009262000 \\
\hline 1 & 2.245775000 & 3.310464000 & 1.953426000 \\
\hline 6 & 0.021940000 & -3.155802000 & 0.983883000 \\
\hline 1 & 0.295252000 & -4.122894000 & 1.425415000 \\
\hline 1 & -0.899995000 & -2.838867000 & 1.481133000 \\
\hline & & & \\
\hline
\end{tabular}




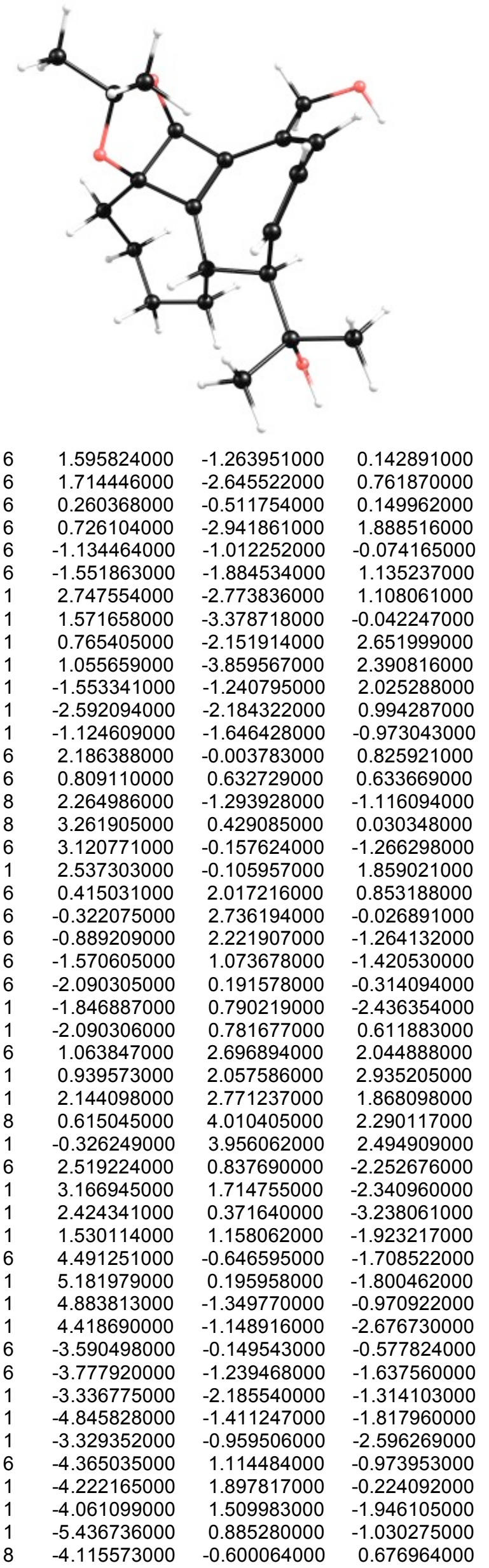


$\mathrm{TS}^{2}(18 \mathrm{a} \rightarrow 24)$

C21H30O4

C1

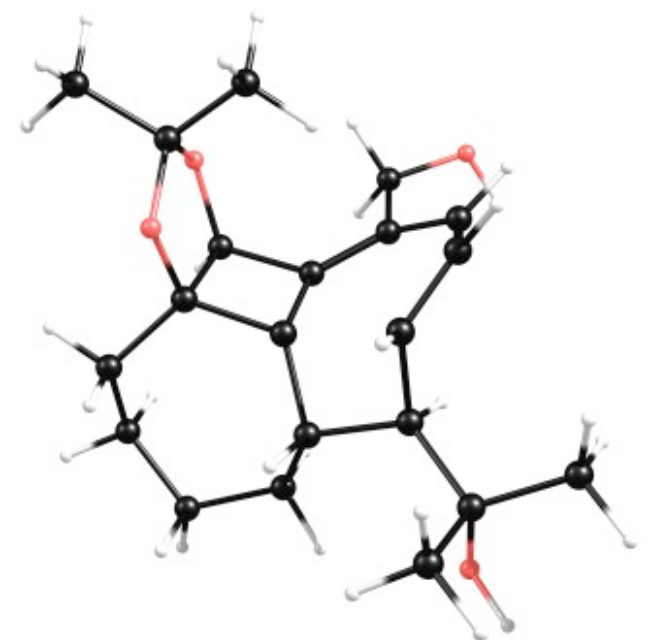

$\begin{array}{rrrr}6 & 1.274443000 & -1.349497000 & -0.175115000 \\ 6 & 1.149877000 & -2.841798000 & 0.104431000 \\ 6 & 0.014037000 & -0.449406000 & -0.081110000 \\ 6 & 0.489935000 & -3.247294000 & 1.429547000 \\ 6 & -1.383150000 & -1.015343000 & 0.128179000 \\ 6 & -1.640837000 & -1.752061000 & 1.440986000 \\ 1 & 2.169792000 & -3.243201000 & 0.075993000 \\ 1 & 0.619072000 & -3.309348000 & -0.736109000 \\ 1 & 0.925528000 & -2.666319000 & 2.254238000 \\ 1 & 0.773305000 & -4.288505000 & 1.627420000 \\ 1 & -1.264795000 & -1.141555000 & 2.273817000 \\ 1 & -2.725709000 & -1.822083000 & 1.572775000 \\ 1 & -1.567982000 & -1.711312000 & -0.704072000 \\ 6 & 1.941145000 & -0.409826000 & 0.868610000 \\ 6 & 0.720608000 & 0.490419000 & 0.734243000 \\ 8 & 2.071425000 & -1.168503000 & -1.335494000 \\ 8 & 3.164643000 & 0.011953000 & 0.311798000 \\ 6 & 3.125907000 & -0.230125000 & -1.098217000 \\ 1 & 2.133893000 & -0.830474000 & 1.860809000 \\ 6 & 0.611867000 & 1.860443000 & 0.916097000 \\ 6 & -0.067133000 & 2.662927000 & -0.031193000 \\ 6 & -0.675523000 & 2.137340000 & -1.159324000 \\ 6 & -1.157468000 & 0.810996000 & -1.221445000 \\ 6 & -2.125607000 & 0.280754000 & -0.167960000 \\ 1 & -1.318664000 & 0.400082000 & -2.220393000 \\ 1 & -2.064161000 & 0.929268000 & 0.714490000 \\ 6 & 1.567975000 & 2.549775000 & 1.880046000 \\ 1 & 1.627645000 & 1.971592000 & 2.814624000 \\ 1 & 2.575981000 & 2.552792000 & 1.450553000 \\ 8 & 1.245446000 & 3.901740000 & 2.135744000 \\ 1 & 0.368049000 & 3.916198000 & 2.536339000 \\ 6 & 2.853111000 & 1.056754000 & -1.866183000 \\ 1 & 3.646966000 & 1.784989000 & -1.676148000 \\ 1 & 2.819880000 & 0.847947000 & -2.939429000 \\ 1 & 1.897157000 & 1.488181000 & -1.564382000 \\ 6 & 4.446741000 & -0.871557000 & -1.496419000 \\ 1 & 5.275588000 & -0.184466000 & -1.305598000 \\ 1 & 4.602311000 & -1.783817000 & -0.916416000 \\ 1 & 4.437391000 & -1.123494000 & -2.560136000 \\ 6 & -3.616828000 & 0.239333000 & -0.564904000 \\ 6 & -3.919580000 & -0.741297000 & -1.700514000\end{array}$


$-1.417710000$

$-4.990556000$

$-0.722734000$

$-1.936246000$

$-3.383580000$

$-0.485006000$

$-4.110829000$

1.642791000

2.338603000

$-3.914637000$

2.025761000

$-5.191603000$

1.628275000

$-4.280948000$

$-0.198488000$

$-5.224743000$

$-0.242242000$

0.161801000

3.725692000

$-0.650618000$

2.738718000

$-1.037640000$

$-3.157225000$

$-1.366371000$

$-3.639967000$

$-1.463376000$

$-3.759534000$

$-2.619750000$

$-0.927162000$

$-0.106516000$

$-1.826865000$

$-1.115362000$

0.628327000

0.432295000

0.008250000

$-2.068366000$

1.497545000

2.426807000

0.681269000

\section{4a}

$\mathrm{C} 21 \mathrm{H} 30 \mathrm{O} 4$

C1

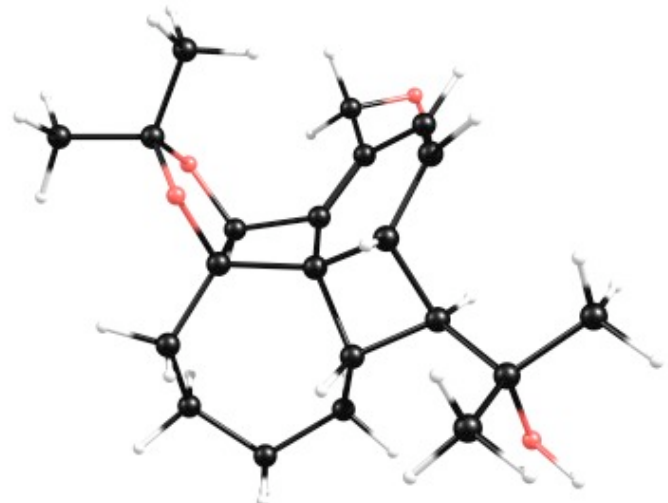

$\begin{array}{rrrr}6 & 1.036092000 & -1.237328000 & -0.287912000 \\ 6 & 0.751200000 & -2.738405000 & -0.167340000 \\ 6 & -0.158733000 & -0.233869000 & -0.113473000 \\ 6 & 0.286646000 & -3.265698000 & 1.205723000 \\ 6 & -1.476622000 & -0.870069000 & 0.371860000 \\ 6 & -1.596201000 & -1.530404000 & 1.732850000 \\ 1 & 1.692136000 & -3.242009000 & -0.416718000 \\ 1 & 0.040111000 & -3.033515000 & -0.950635000 \\ 1 & 0.977151000 & -2.905753000 & 1.979206000 \\ 1 & 0.423826000 & -4.354316000 & 1.189554000 \\ 1 & -1.005596000 & -0.979557000 & 2.477876000 \\ 1 & -2.640637000 & -1.484940000 & 2.060577000 \\ 1 & -1.706572000 & -1.633736000 & -0.386567000 \\ 6 & 1.789275000 & -0.522353000 & 0.874657000 \\ 6 & 0.750602000 & 0.577919000 & 0.764456000 \\ 8 & 1.853075000 & -0.958650000 & -1.410591000 \\ 8 & 3.087630000 & -0.244682000 & 0.403892000 \\ 6 & 3.132187000 & -0.458543000 & -1.012000000 \\ 1 & 1.864463000 & -1.053524000 & 1.827189000 \\ 6 & 0.914624000 & 1.916104000 & 0.731806000 \\ 6 & 0.123496000 & 2.710980000 & -0.207396000 \\ 6 & -0.655349000 & 2.127103000 & -1.138477000 \\ 6 & -1.035320000 & 0.687366000 & -1.061743000 \\ 6 & -2.213981000 & 0.401490000 & -0.069300000 \\ 1 & -1.220549000 & 0.244591000 & -2.046965000 \\ 1 & -2.164370000 & 1.149414000 & 0.730896000 \\ 6 & 2.052340000 & 2.612655000 & 1.437867000 \\ 1 & 2.583642000 & 1.909077000 & 2.092293000 \\ 1 & 2.781493000 & 2.986958000 & 0.710090000 \\ 8 & 1.606319000 & 3.761317000 & 2.146336000 \\ 1 & 0.930713000 & 3.464251000 & 2.767422000 \\ 6 & 3.381993000 & 0.853410000 & -1.741296000 \\ 1 & 4.326436000 & 1.294344000 & -1.410710000 \\ 1 & 3.436192000 & 0.679051000 & -2.819643000 \\ 1 & 2.566276000 & 1.550474000 & -1.541076000 \\ 6 & 4.213033000 & -1.495200000 & -1.296415000\end{array}$


4.243655000

$-1.728002000$

$-3.671138000$

0.347113000

$-2.364226000$

$-3.934881000$

$-0.720520000$

$-0.542852000$

$-3.725518000$

$-1.720989000$

$-4.988032000$

$-0.693975000$

$-1.607391000$

$-1.220468000$

$-1.912388000$

$-3.333953000$

$-0.560512000$

$-2.508102000$

$-4.115675000$

1.722279000

$-1.052223000$

$-3.939585000$

2.487363000

$-3.579870000$

2.008298000

1.714585000

$-0.290955000$

$-5.186699000$

0.020704000

$-4.400194000$

$-0.030438000$

$-1.289845000$

0.648403000

0.410806000

0.306102000

3.782290000

2.728495000

227917000

$-1.084280000$

$-2.998152000$

$-1.157036000$

$-3.475513000$

$-1.937237000$

1.655778000

2.634149000

$-1.839859000$

$-3.518901000$

0.968859000

\section{$X=4 \quad(r, r)$}

t4

\section{$\mathrm{C} 18 \mathrm{H} 24 \mathrm{O} 4$}

C1

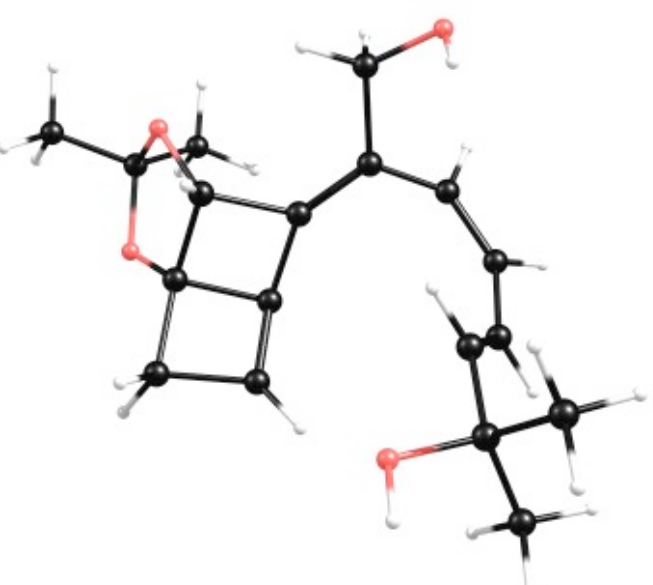

$\begin{array}{rrrr}6 & 1.680954000 & -0.308795000 & -0.982677000 \\ 6 & 0.676061000 & 0.407602000 & -0.020397000 \\ 8 & 3.006260000 & -1.603434000 & 0.464909000 \\ 8 & 3.010687000 & 0.133978000 & -1.034093000 \\ 6 & 3.724660000 & -0.435466000 & 0.074665000 \\ 6 & 5.096297000 & -0.855271000 & -0.421943000 \\ 1 & 5.656776000 & 0.016638000 & -0.768806000 \\ 1 & 5.656812000 & -1.335758000 & 0.383979000 \\ 1 & 4.984521000 & -1.559986000 & -1.248503000 \\ 6 & 3.795015000 & 0.553160000 & 1.233875000 \\ 1 & 4.320677000 & 1.460739000 & 0.923575000 \\ 1 & 2.792116000 & 0.825357000 & 1.567998000 \\ 1 & 4.333041000 & 0.103967000 & 2.073272000 \\ 6 & 0.310720000 & 1.696394000 & 0.113772000 \\ 6 & -2.803261000 & 0.234192000 & 0.090413000 \\ 6 & -2.631475000 & 0.762387000 & 1.311708000 \\ 6 & -0.517128000 & 2.222504000 & 1.203460000 \\ 6 & -1.684641000 & 1.795110000 & 1.729032000 \\ 6 & -3.910247000 & -0.705560000 & -0.323518000 \\ 6 & -4.701940000 & -1.288442000 & 0.847203000 \\ 1 & -4.039461000 & -1.815150000 & 1.539217000 \\ 1 & -5.244688000 & -0.515066000 & 1.398760000 \\ 1 & -5.444312000 & -2.003055000 & 0.473875000 \\ 6 & -4.847061000 & 0.026829000 & -1.297857000 \\ 1 & -4.282398000 & 0.406928000 & -2.153936000 \\ 1 & -5.619699000 & -0.655776000 & -1.674446000\end{array}$




$\begin{array}{lrrr}1 & -5.348849000 & 0.867715000 & -0.810343000 \\ 8 & -3.243554000 & -1.766594000 & -1.027553000 \\ 1 & -3.928068000 & -2.351105000 & -1.376800000 \\ 6 & 0.925932000 & 2.748211000 & -0.784726000 \\ 1 & 1.638135000 & 3.349966000 & -0.205241000 \\ 1 & 1.497871000 & 2.273896000 & -1.593562000 \\ 8 & -0.025609000 & 3.677140000 & -1.277660000 \\ 1 & -0.718472000 & 3.170574000 & -1.716664000 \\ 1 & -0.106811000 & 3.129962000 & 1.650322000 \\ 1 & -2.023317000 & 2.350629000 & 2.603732000 \\ 1 & 1.297005000 & -0.440923000 & -1.999376000 \\ 1 & -2.136291000 & 0.512186000 & -0.723429000 \\ 6 & 0.492298000 & -0.896835000 & 0.631532000 \\ 6 & -0.271468000 & -2.011539000 & 0.482024000 \\ 6 & 0.878524000 & -2.852996000 & -0.081609000 \\ 6 & 1.695435000 & -1.546286000 & -0.019483000 \\ 1 & 0.682162000 & -3.237895000 & -1.090113000 \\ 1 & 1.274738000 & -3.668155000 & 0.534562000 \\ 1 & -3.332386000 & 0.472534000 & 2.093641000 \\ 1 & -1.343428000 & -2.158134000 & 0.400631000 \\ \text { TS } & & & \\ \text { C18H2 } & & & \\ \text { C1 } & & & \end{array}$

C

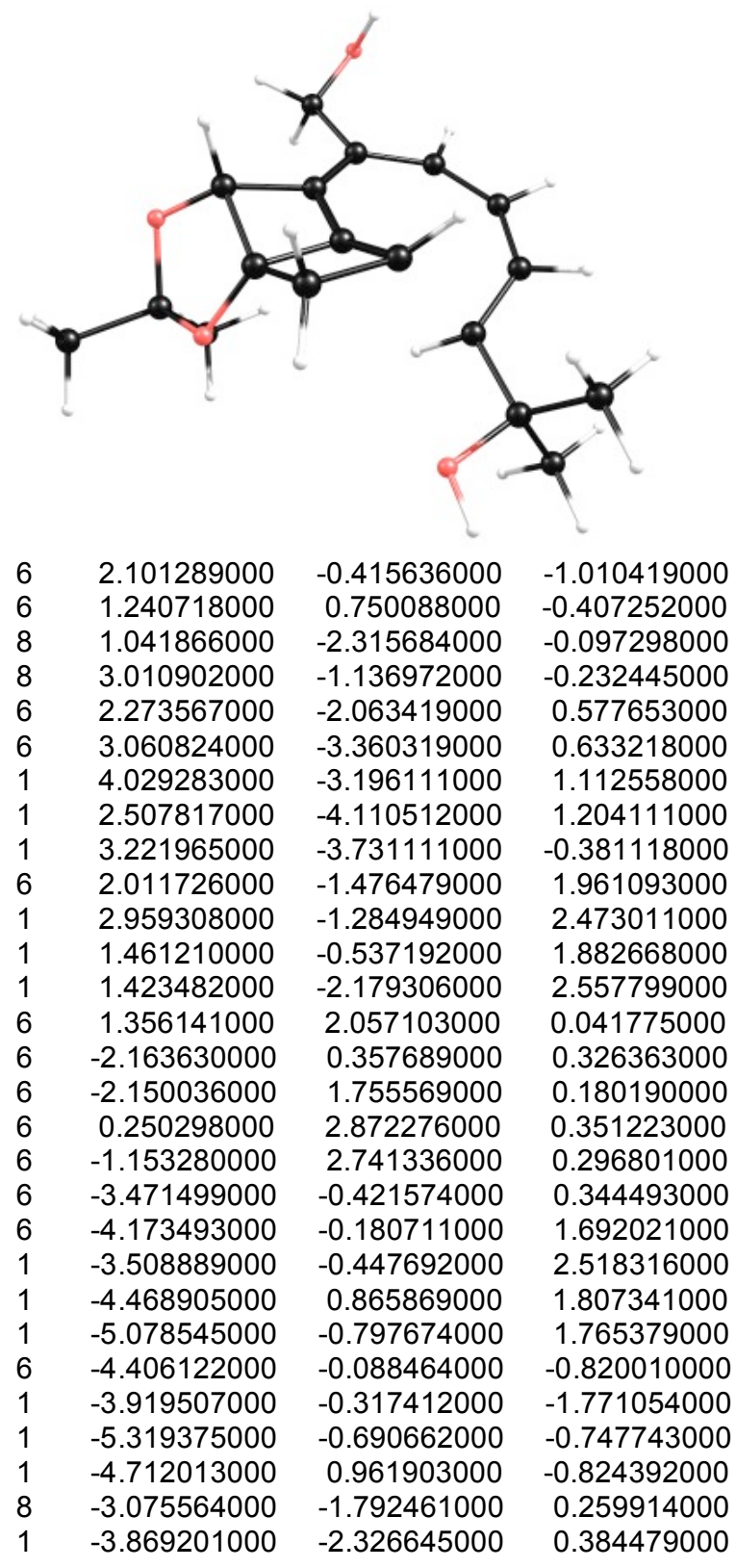


3.454119000 2.098263000

$-0.426809000$

2.837576000 4.035442000 0.042335000

2.521689000 4.225896000 $-0.849053000$

0.558105000 3.889326000

$-1.613301000$ 3.731253000 0.591012000

2.566232000

$-0.154776000$

$-1.436037000$

$-0.109234000$

0.326483000

0.120856000

$-0.004767000$

$-1.034433000$

$-0.191845000$

$-0.452675000$

$-1.562765000$

$-1.965459000$

0.980545000

$-0.721458000$

$-1.469494000$

0.763343000

$-1.298732000$

$-1.015228000$

$-2.458657000$

$-1.970582000$

$-1.024106000$

$-0.249475000$

$-1.565600000$

$-1.695601000$

$-3.113741000$

2.186940000

$-3.047351000$

$-1.548370000$

0.535037000

$-0.093472000$

$-2.089212000$

c4

$\mathrm{C} 18 \mathrm{H} 24 \mathrm{O} 4$

C1

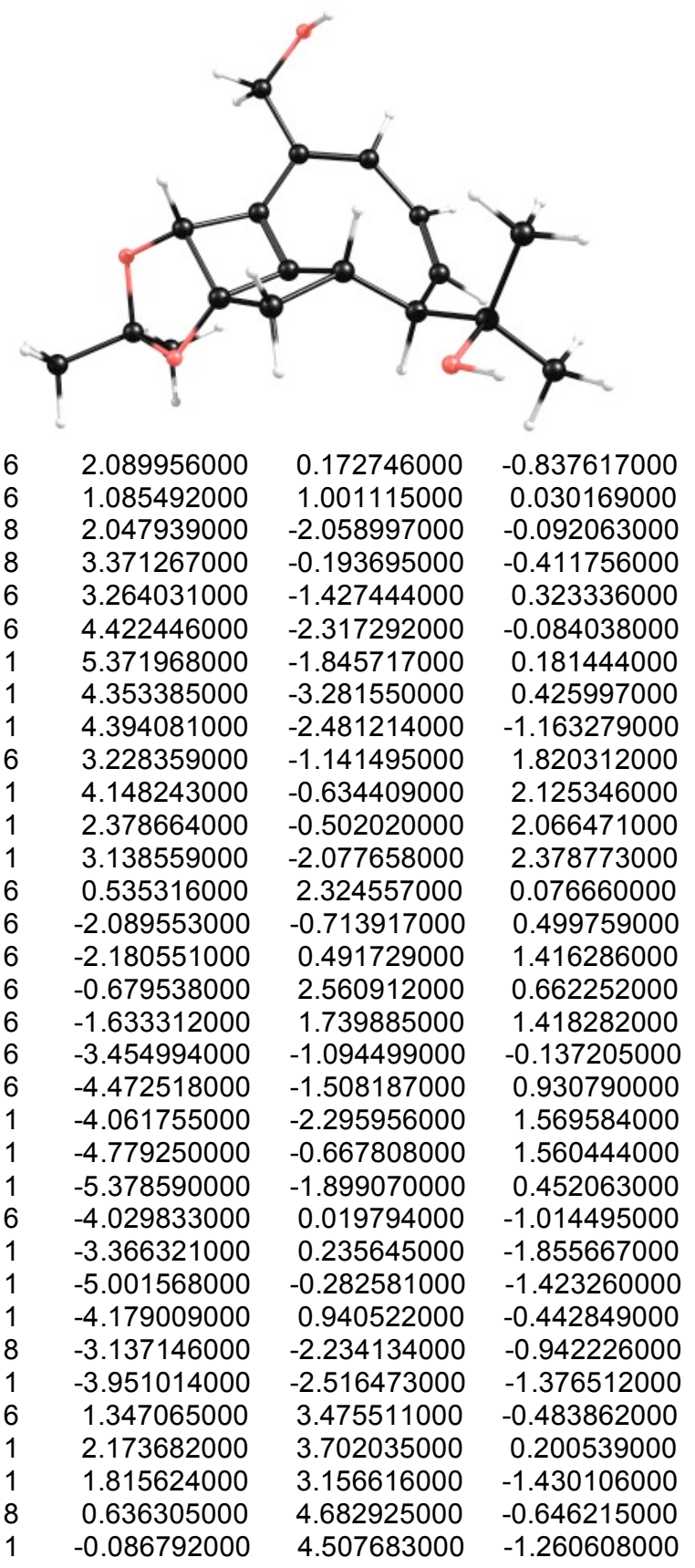




$\begin{array}{rrrr}1 & -0.940469000 & 3.618744000 & 0.706714000 \\ 1 & -2.071718000 & 2.357147000 & 2.206707000 \\ 1 & 2.129234000 & 0.558903000 & -1.864115000 \\ 1 & -1.810453000 & -1.580619000 & 1.116960000 \\ 6 & 0.325253000 & -0.110505000 & 0.193068000 \\ 6 & -0.969493000 & -0.446771000 & -0.482894000 \\ 6 & -0.129168000 & -1.514891000 & -1.285659000 \\ 6 & 1.190009000 & -1.070295000 & -0.575689000 \\ 1 & -0.183682000 & -1.435144000 & -2.374608000 \\ 1 & -0.364310000 & -2.538214000 & -0.982992000 \\ 1 & -2.901202000 & 0.348684000 & 2.221836000 \\ 1 & -1.268028000 & 0.397937000 & -1.117542000\end{array}$

\section{TS2}

$\mathrm{C} 18 \mathrm{H} 24 \mathrm{O} 4$

C1

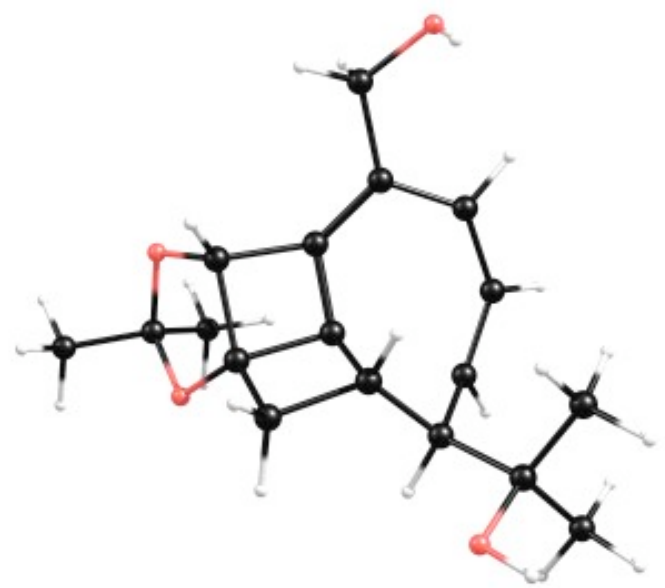

$\begin{array}{rrrr}6 & 2.028443000 & 0.196763000 & -0.927576000 \\ 6 & 0.961589000 & 0.994722000 & -0.142510000 \\ 8 & 2.103420000 & -2.013782000 & -0.126295000 \\ 8 & 3.307272000 & -0.072017000 & -0.413119000 \\ 6 & 3.243164000 & -1.288592000 & 0.349500000 \\ 6 & 4.484602000 & -2.102942000 & 0.041439000 \\ 1 & 5.379313000 & -1.559108000 & 0.354634000 \\ 1 & 4.449543000 & -3.057981000 & 0.571846000 \\ 1 & 4.539285000 & -2.292237000 & -1.032590000 \\ 6 & 3.086908000 & -0.973684000 & 1.833084000 \\ 1 & 3.937101000 & -0.379531000 & 2.179778000 \\ 1 & 2.169214000 & -0.409397000 & 2.009856000 \\ 1 & 3.044444000 & -1.901394000 & 2.410837000 \\ 6 & 0.438863000 & 2.270438000 & 0.019955000 \\ 6 & -1.951246000 & -0.947959000 & 0.283874000 \\ 6 & -1.568189000 & 0.008287000 & 1.422557000 \\ 6 & -0.727892000 & 2.402704000 & 0.794577000 \\ 6 & -1.430337000 & 1.407871000 & 1.505726000 \\ 6 & -3.453309000 & -1.006048000 & -0.080134000 \\ 6 & -4.288796000 & -1.413295000 & 1.137226000 \\ 1 & -3.923899000 & -2.356980000 & 1.554243000 \\ 1 & -4.262181000 & -0.649554000 & 1.920661000 \\ 1 & -5.337870000 & -1.552537000 & 0.849829000 \\ 6 & -3.982526000 & 0.300054000 & -0.674712000 \\ 1 & -3.476473000 & 0.533972000 & -1.614784000 \\ 1 & -5.055485000 & 0.211201000 & -0.885407000 \\ 1 & -3.845976000 & 1.139509000 & 0.012696000 \\ 8 & -3.501201000 & -2.043697000 & -1.065057000 \\ 1 & -4.412269000 & -2.104596000 & -1.376544000 \\ 6 & 1.186151000 & 3.512446000 & -0.420523000 \\ 1 & 1.924282000 & 3.793645000 & 0.339817000 \\ 1 & 1.755005000 & 3.288644000 & -1.337215000 \\ 8 & 0.360367000 & 4.647861000 & -0.585751000 \\ 1 & -0.323050000 & 4.413362000 & -1.224958000 \\ 1 & -0.979404000 & 3.428025000 & 1.062073000 \\ 1 & -1.850107000 & 1.811340000 & 2.432802000 \\ & & & \end{array}$


f4 $\mathrm{C} 18 \mathrm{H} 24 \mathrm{O} 4$

C1

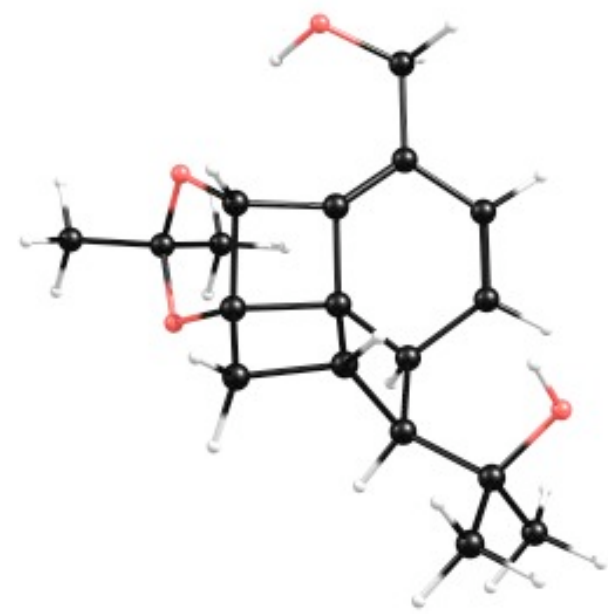

$\begin{array}{rrrr}6 & -0.152521000 & -0.106742000 & -0.072646000 \\ 6 & 0.806326000 & -1.271171000 & -0.523811000 \\ 6 & -0.310296000 & -1.928732000 & -1.346429000 \\ 6 & -1.216944000 & -0.703639000 & -1.031284000 \\ 6 & -1.168459000 & 0.116014000 & 1.036376000 \\ 6 & -1.347443000 & 1.617046000 & 1.159125000 \\ 6 & -0.455448000 & 2.513213000 & 0.683608000 \\ 6 & 0.760544000 & 2.170519000 & -0.087225000 \\ 6 & 0.837977000 & 0.895166000 & -0.489495000 \\ 1 & -0.955911000 & -0.300261000 & 2.032303000 \\ 1 & -2.197612000 & 1.983513000 & 1.727479000 \\ 1 & -0.627415000 & 3.568498000 & 0.891238000 \\ 6 & 1.738765000 & -0.155942000 & -1.123143000 \\ 8 & 3.001079000 & -0.395988000 & -0.523904000 \\ 8 & 1.636465000 & -2.066477000 & 0.256582000 \\ 6 & 2.844082000 & -1.354576000 & 0.534940000 \\ 6 & 2.750350000 & -0.651780000 & 1.885432000 \\ 1 & 2.601977000 & -1.387878000 & 2.680328000 \\ 1 & 3.673920000 & -0.101506000 & 2.086826000 \\ 1 & 1.916864000 & 0.053456000 & 1.899130000 \\ 6 & 4.000162000 & -2.333664000 & 0.458783000 \\ 1 & 3.893730000 & -3.103884000 & 1.226989000 \\ 1 & 4.011386000 & -2.808018000 & -0.524512000 \\ 1 & 4.947654000 & -1.811535000 & 0.613811000 \\ 6 & -2.181237000 & -0.756634000 & 0.203362000 \\ 1 & -1.566244000 & -0.126213000 & -1.894086000 \\ 1 & -2.187021000 & -1.774150000 & 0.615318000 \\ 6 & -3.645307000 & -0.329706000 & 0.026494000 \\ 6 & -4.328487000 & -1.228433000 & -1.011950000 \\ 1 & -3.830212000 & -1.156591000 & -1.985157000 \\ 1 & -4.315691000 & -2.280075000 & -0.706814000 \\ 1 & -5.368768000 & -0.916664000 & -1.142136000 \\ 6 & -4.383090000 & -0.406415000 & 1.361993000 \\ 1 & -3.890501000 & 0.212720000 & 2.116492000 \\ 1 & -5.406782000 & -0.040865000 & 1.240251000 \\ 1 & -4.421916000 & -1.436298000 & 1.729162000 \\ 6 & 1.835486000 & 3.195903000 & -0.314918000\end{array}$




\section{$X=4 \quad(r, s)$}

\section{t4}

C18H24O4

C1

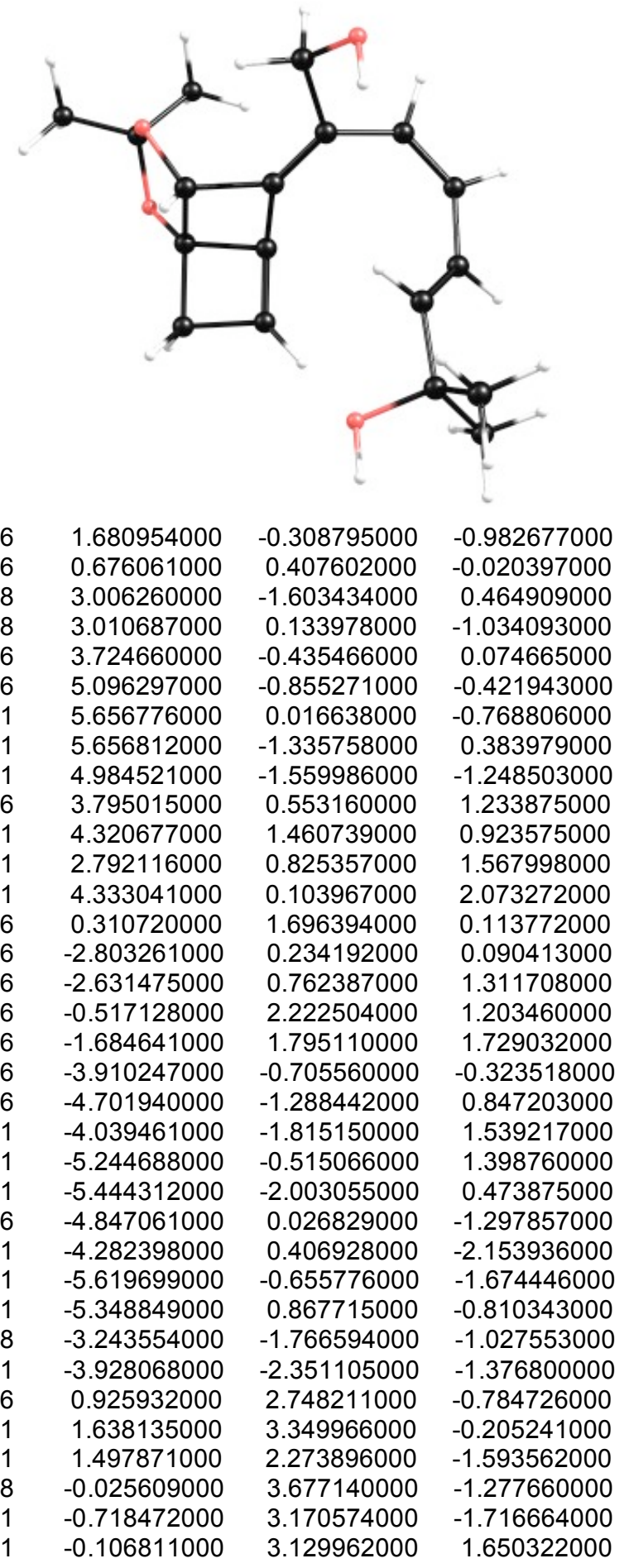




\section{$\mathrm{TS}^{1}$}

\section{$\mathrm{C} 18 \mathrm{H} 24 \mathrm{O} 4$}

C1

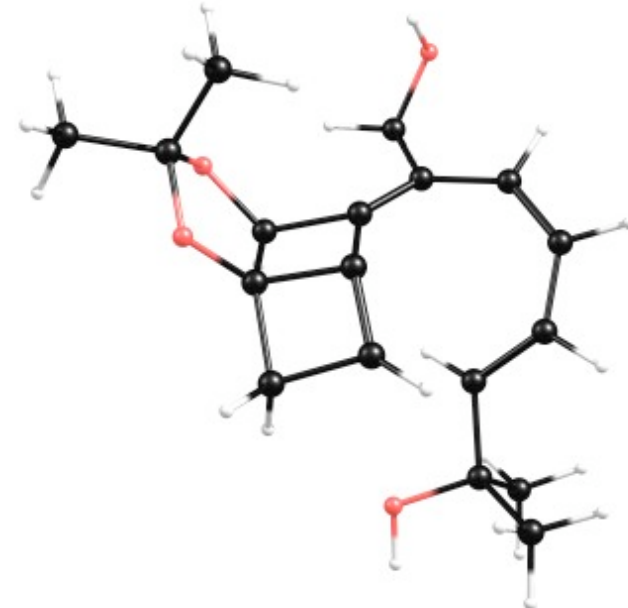

$\begin{array}{rrrr}6 & -1.500441000 & -0.289885000 & -0.986341000 \\ 6 & -0.715853000 & 0.669513000 & -0.033137000 \\ 8 & -2.272322000 & -2.013350000 & 0.413285000 \\ 8 & -2.906318000 & -0.315100000 & -0.994955000 \\ 6 & -3.345145000 & -1.126783000 & 0.104743000 \\ 6 & -3.685036000 & -0.254708000 & 1.310491000 \\ 1 & -4.487181000 & 0.443151000 & 1.052582000 \\ 1 & -4.017687000 & -0.880854000 & 2.143245000 \\ 1 & -2.811087000 & 0.315434000 & 1.631497000 \\ 6 & -4.525493000 & -1.955679000 & -0.368406000 \\ 1 & -5.354787000 & -1.304037000 & -0.655266000 \\ 1 & -4.226209000 & -2.553878000 & -1.231446000 \\ 1 & -4.861685000 & -2.622593000 & 0.429645000 \\ 6 & -0.424396000 & 2.017831000 & -0.137763000 \\ 6 & 2.399853000 & 0.144630000 & 0.041019000 \\ 6 & 2.473308000 & 1.090884000 & 1.045517000 \\ 6 & 0.518559000 & 2.703100000 & 0.663438000 \\ 6 & 1.669839000 & 2.236049000 & 1.273933000 \\ 6 & 3.589234000 & -0.712054000 & -0.355970000 \\ 6 & 4.504723000 & 0.126777000 & -1.265923000 \\ 1 & 3.943623000 & 0.500377000 & -2.126891000 \\ 1 & 4.923661000 & 0.981173000 & -0.727412000 \\ 1 & 5.338355000 & -0.483787000 & -1.637151000 \\ 6 & 4.386334000 & -1.274307000 & 0.824054000 \\ 1 & 3.755073000 & -1.900473000 & 1.459519000 \\ 1 & 5.208404000 & -1.895683000 & 0.450532000 \\ 1 & 4.832626000 & -0.486562000 & 1.437018000 \\ 8 & 3.043304000 & -1.792937000 & -1.119399000 \\ 1 & 3.785888000 & -2.291381000 & -1.480981000 \\ 6 & -1.269524000 & 2.863489000 & -1.081945000 \\ 1 & -0.646970000 & 3.274551000 & -1.884662000 \\ 1 & -2.046358000 & 2.241634000 & -1.545371000 \\ 8 & -1.835609000 & 3.995153000 & -0.437155000 \\ 1 & -2.404838000 & 3.660757000 & 0.266289000 \\ 1 & 0.304074000 & 3.762448000 & 0.803063000 \\ 1 & 2.108533000 & 2.928876000 & 1.992983000\end{array}$


c4 $\mathrm{C} 18 \mathrm{H} 24 \mathrm{O} 4$

C1

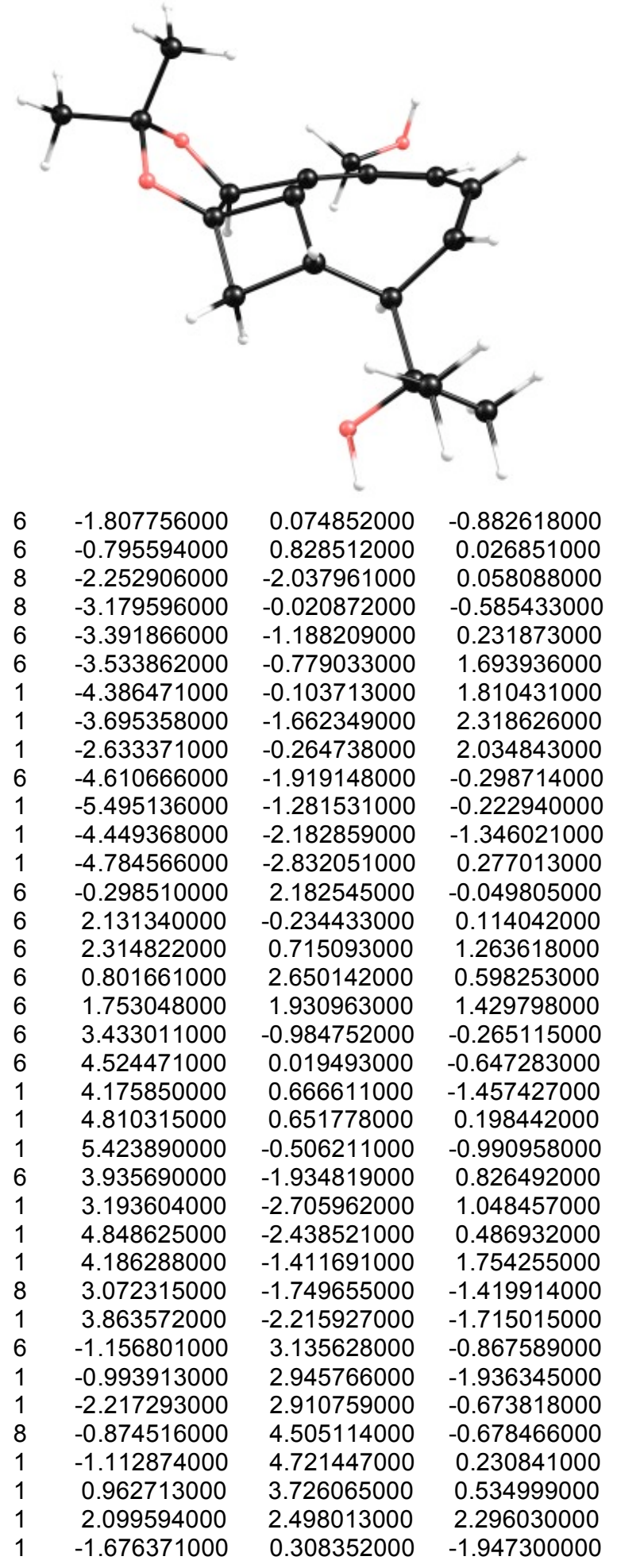




$\begin{array}{rrrr}1 & 1.854299000 & 0.351578000 & -0.773031000 \\ 6 & -0.333292000 & -0.349657000 & 0.544584000 \\ 6 & 0.927034000 & -1.148705000 & 0.409126000 \\ 6 & 0.151037000 & -1.894609000 & -0.758220000 \\ 6 & -1.188406000 & -1.223139000 & -0.334253000 \\ 1 & 0.132299000 & -2.984385000 & -0.674977000 \\ 1 & 0.511385000 & -1.607711000 & -1.747645000 \\ 1 & 2.996029000 & 0.389935000 & 2.049448000 \\ 1 & 1.131730000 & -1.830006000 & 1.241102000\end{array}$

$\mathrm{TS}^{2}$

C18H24O4

C1

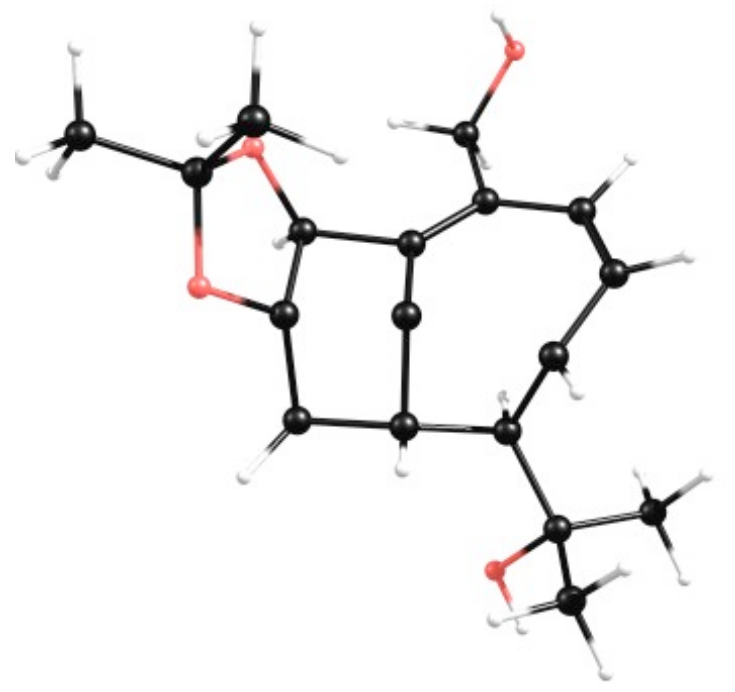

$\begin{array}{rrrr}6 & -1.749690000 & -0.035160000 & -0.990629000 \\ 6 & -0.620535000 & 0.739918000 & -0.319516000 \\ 8 & -2.204541000 & -2.100281000 & 0.010971000 \\ 8 & -3.048141000 & 0.005952000 & -0.423014000 \\ 6 & -3.266371000 & -1.189389000 & 0.345216000 \\ 6 & -3.216984000 & -0.859261000 & 1.831509000 \\ 1 & -3.976324000 & -0.109522000 & 2.070367000 \\ 1 & -3.407663000 & -1.756557000 & 2.427028000 \\ 1 & -2.235530000 & -0.459906000 & 2.095761000 \\ 6 & -4.577147000 & -1.816897000 & -0.088385000 \\ 1 & -5.404824000 & -1.132282000 & 0.113525000 \\ 1 & -4.545212000 & -2.029692000 & -1.159081000 \\ 1 & -4.751204000 & -2.748968000 & 0.455785000 \\ 6 & -0.292173000 & 2.079487000 & -0.289679000 \\ 6 & 2.113108000 & -0.250655000 & -0.080458000 \\ 6 & 1.566231000 & 0.344499000 & 1.234061000 \\ 6 & 0.654280000 & 2.562981000 & 0.655181000 \\ 6 & 1.385815000 & 1.737437000 & 1.476421000 \\ 6 & 3.588698000 & -0.684544000 & -0.125457000 \\ 6 & 4.505445000 & 0.537508000 & -0.018879000 \\ 1 & 4.274830000 & 1.254655000 & -0.811804000 \\ 1 & 4.391397000 & 1.043064000 & 0.944808000 \\ 1 & 5.556893000 & 0.240647000 & -0.118977000 \\ 6 & 3.958264000 & -1.724678000 & 0.935062000 \\ 1 & 3.347049000 & -2.625340000 & 0.832213000 \\ 1 & 5.007917000 & -2.019268000 & 0.816764000 \\ 1 & 3.845757000 & -1.337030000 & 1.952341000 \\ 8 & 3.710501000 & -1.270542000 & -1.426054000 \\ 1 & 4.631291000 & -1.536878000 & -1.536530000 \\ 6 & -1.102167000 & 3.085393000 & -1.080314000 \\ 1 & -0.484150000 & 3.514782000 & -1.877882000 \\ 1 & -1.950501000 & 2.581701000 & -1.564932000 \\ 8 & -1.533873000 & 4.196225000 & -0.310072000 \\ 1 & -2.089584000 & 3.845926000 & 0.396500000 \\ 1 & 0.623928000 & 3.630637000 & 0.861889000 \\ 1 & 1.729653000 & 2.142051000 & 2.429099000\end{array}$


f4 (dissociation upon optimization) $\mathrm{C} 18 \mathrm{H} 24 \mathrm{O} 4$

C1

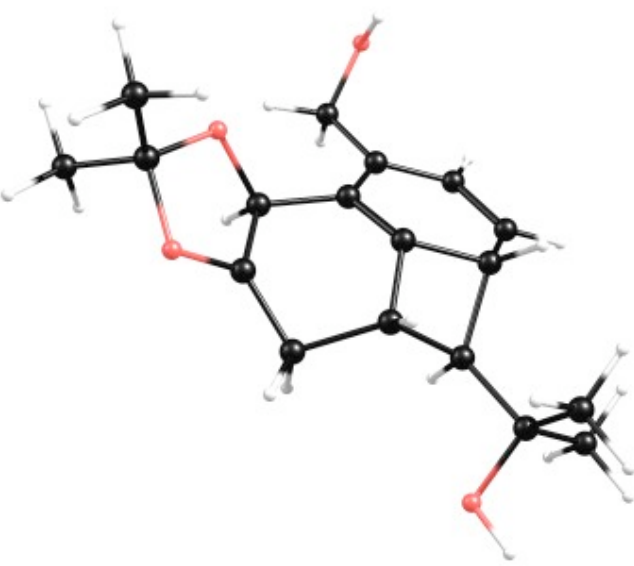

$\begin{array}{rrrr}6 & -1.694997000 & -0.057494000 & -0.481348000 \\ 6 & -0.650959000 & 0.887588000 & 0.014571000 \\ 8 & -2.153726000 & -2.302564000 & -0.200886000 \\ 8 & -2.858437000 & -0.192737000 & 0.361570000 \\ 6 & -3.333568000 & -1.505212000 & 0.206860000 \\ 6 & -3.808219000 & -2.027852000 & 1.544910000 \\ 1 & -4.653019000 & -1.426593000 & 1.890375000 \\ 1 & -4.127075000 & -3.069687000 & 1.462020000 \\ 1 & -3.000082000 & -1.949406000 & 2.274753000 \\ 6 & -4.358722000 & -1.628512000 & -0.910578000 \\ 1 & -5.251098000 & -1.054466000 & -0.648757000 \\ 1 & -3.956971000 & -1.236616000 & -1.848392000 \\ 1 & -4.640768000 & -2.673614000 & -1.061750000 \\ 6 & -0.681431000 & 2.278890000 & -0.192202000 \\ 6 & 2.372712000 & -0.216679000 & -0.232705000 \\ 6 & 1.802947000 & 0.974358000 & 0.672770000 \\ 6 & 0.562992000 & 2.989998000 & -0.046263000 \\ 6 & 1.753070000 & 2.413331000 & 0.278773000 \\ 6 & 3.774396000 & -0.805209000 & -0.041223000 \\ 6 & 4.831236000 & 0.218424000 & -0.466078000 \\ 1 & 4.663346000 & 0.531805000 & -1.500430000 \\ 1 & 4.808817000 & 1.106508000 & 0.173331000 \\ 1 & 5.837546000 & -0.213618000 & -0.397670000 \\ 6 & 4.047443000 & -1.290835000 & 1.384591000 \\ 1 & 3.337486000 & -2.067722000 & 1.680251000 \\ 1 & 5.055077000 & -1.720067000 & 1.446149000 \\ 1 & 3.997183000 & -0.475496000 & 2.112633000 \\ 8 & 3.791153000 & -1.924533000 & -0.937182000 \\ 1 & 4.665798000 & -2.328606000 & -0.878049000 \\ 6 & -1.906648000 & 3.030792000 & -0.614909000 \\ 1 & -1.773576000 & 3.509620000 & -1.594248000 \\ 1 & -2.761248000 & 2.341981000 & -0.706686000 \\ 8 & -2.221237000 & 4.113809000 & 0.263419000 \\ 1 & -2.180440000 & 3.750297000 & 1.156653000 \\ 1 & 0.538036000 & 4.057253000 & -0.260160000 \\ 1 & 2.660171000 & 3.013005000 & 0.302532000 \\ 1 & -2.029283000 & 0.269406000 & -1.492117000 \\ 1 & 2.276027000 & 0.083681000 & -1.282523000 \\ 6 & 0.483914000 & 0.282827000 & 0.511714000\end{array}$




$\begin{array}{rrrr}6 & 1.092789000 & -1.020342000 & 0.171612000 \\ 6 & 0.186702000 & -1.854589000 & -0.803900000 \\ 6 & -1.179387000 & -1.448424000 & -0.483268000 \\ 1 & 0.315702000 & -2.934890000 & -0.698219000 \\ 1 & 0.469347000 & -1.579527000 & -1.829465000 \\ 1 & 2.229902000 & 0.849154000 & 1.684479000 \\ 1 & 1.291479000 & -1.660561000 & 1.039967000\end{array}$

$$
X=5 \quad(r, r)
$$

t5 C19H26O4

C1

$$
\begin{aligned}
& \text { (2) } \\
& 6 \quad 1.626910000 \\
& -1.044618000 \\
& 0.693272000 \\
& 0.619912000 \\
& 0.003549000 \\
& 2.815229000 \\
& -1.501713000 \\
& 0.341748000 \\
& 2.987936000 \\
& 0.301276000 \\
& -1.064637000 \\
& 3.635716000 \\
& 4.969963000 \\
& -0.386248000 \\
& 0.013126000 \\
& 5.611067000 \\
& 5.478167000 \\
& -0.501933000 \\
& -0.067319000 \quad-0.798891000 \\
& 4.801174000 \\
& -1.475376000 \\
& 0.277323000 \\
& -1.546481000-1.366912000 \\
& 3.795333000 \\
& 0.530573000 \\
& 1.222963000 \\
& 1.396915000 \\
& 0.880619000 \\
& 2.821908000 \\
& 4.280901000 \\
& -0.012652000 \\
& 1.910722000 \\
& 0.268235000 \\
& 0.992534000 \\
& 2.413832000 \\
& 1.994526000 \\
& -0.655361000 \\
& -1.078882000 \\
& -1.569345000
\end{aligned}
$$


C1

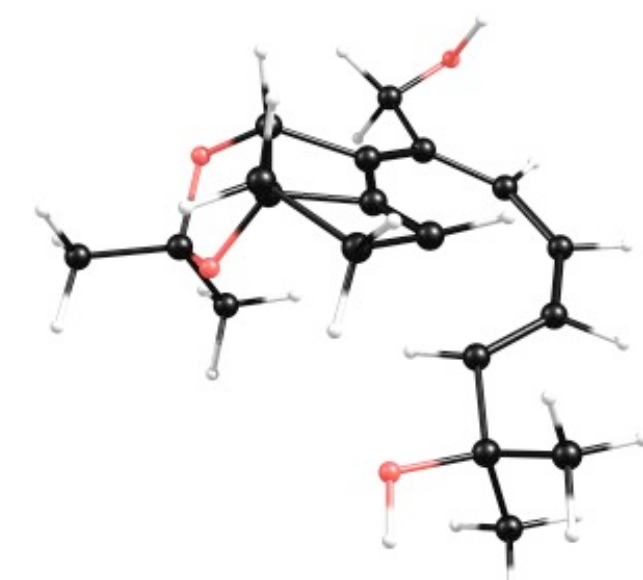

$\begin{array}{rrrr}6 & -2.186349000 & -0.539679000 & 0.866989000 \\ 6 & -1.261106000 & 0.675869000 & 0.597122000 \\ 8 & -0.858021000 & -2.155908000 & -0.167705000 \\ 8 & -2.912855000 & -1.120507000 & -0.182661000 \\ 6 & -2.004136000 & -1.890663000 & -0.976140000 \\ 6 & -2.680135000 & -3.212072000 & -1.306938000 \\ 1 & -3.582920000 & -3.039856000 & -1.899200000 \\ 1 & -2.000823000 & -3.850350000 & -1.877956000 \\ 1 & -2.953854000 & -3.721816000 & -0.380655000 \\ 6 & -1.597183000 & -1.120126000 & -2.228577000 \\ 1 & -2.472514000 & -0.933938000 & -2.857702000 \\ 1 & -1.149837000 & -0.161195000 & -1.960467000 \\ 1 & -0.868428000 & -1.699781000 & -2.802212000 \\ 6 & -1.462855000 & 1.970988000 & 0.131639000 \\ 6 & 2.003379000 & 0.628068000 & -0.358950000 \\ 6 & 1.981587000 & 2.005249000 & -0.120604000 \\ 6 & -0.466063000 & 2.928938000 & -0.119539000 \\ 6 & 0.937786000 & 2.941869000 & -0.105071000 \\ 6 & 3.320822000 & -0.094910000 & -0.601653000 \\ 6 & 3.880451000 & 0.338881000 & -1.968046000 \\ 1 & 3.143765000 & 0.152491000 & -2.754183000 \\ 1 & 4.136176000 & 1.402025000 & -1.976348000 \\ 1 & 4.788926000 & -0.230278000 & -2.205291000 \\ 6 & 4.359147000 & 0.130363000 & 0.500634000 \\ 1 & 3.978439000 & -0.223788000 & 1.461411000 \\ 1 & 5.276450000 & -0.423497000 & 0.268463000 \\ 1 & 4.635823000 & 1.183997000 & 0.601078000 \\ 8 & 2.977651000 & -1.481434000 & -0.662710000 \\ 1 & 3.776732000 & -1.963196000 & -0.907583000 \\ 6 & -2.907371000 & 2.384677000 & -0.133695000 \\ 1 & -3.160600000 & 2.180168000 & -1.180484000 \\ 1 & -3.582673000 & 1.765130000 & 0.474271000 \\ 8 & -3.167750000 & 3.762760000 & 0.053825000 \\ 1 & -2.959128000 & 3.969655000 & 0.972697000 \\ 1 & -0.892579000 & 3.912209000 & -0.315909000 \\ 1 & 1.321400000 & 3.963523000 & -0.089620000 \\ 1 & -2.841167000 & -0.438640000 & 1.738050000 \\ & & & \end{array}$


c5

$\mathrm{C} 19 \mathrm{H} 26 \mathrm{O} 4$

C1

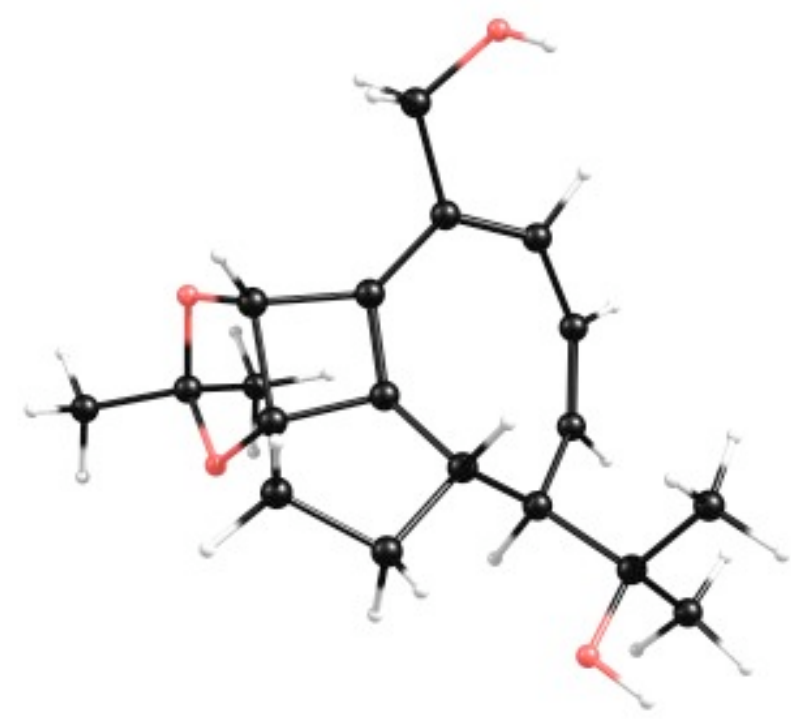

$\begin{array}{rrrr}6 & 2.295858000 & 0.097078000 & -0.872049000 \\ 6 & 1.098660000 & 0.908226000 & -0.334237000 \\ 8 & 1.957492000 & -2.057077000 & 0.018394000 \\ 8 & 3.368183000 & -0.238723000 & -0.030331000 \\ 6 & 2.996785000 & -1.393099000 & 0.739313000 \\ 6 & 4.198148000 & -2.320827000 & 0.793288000 \\ 1 & 5.031466000 & -1.830683000 & 1.303432000 \\ 1 & 3.944113000 & -3.236355000 & 1.333833000 \\ 1 & 4.503719000 & -2.579135000 & -0.222756000 \\ 6 & 2.518176000 & -0.981588000 & 2.129274000 \\ 1 & 3.315210000 & -0.447133000 & 2.654356000 \\ 1 & 1.644265000 & -0.332169000 & 2.063617000 \\ 1 & 2.251512000 & -1.870751000 & 2.708240000 \\ 6 & 0.743595000 & 2.282765000 & -0.116183000 \\ 6 & -2.051718000 & -0.525620000 & 0.495901000 \\ 6 & -1.842609000 & 0.613853000 & 1.470481000 \\ 6 & -0.347631000 & 2.620871000 & 0.621006000 \\ 6 & -1.229841000 & 1.823491000 & 1.470138000 \\ 6 & -3.566547000 & -0.662192000 & 0.136803000 \\ 6 & -4.426939000 & -0.867592000 & 1.389829000 \\ 1 & -4.033867000 & -1.685968000 & 2.001073000 \\ 1 & -4.496993000 & 0.033256000 & 2.005447000 \\ 1 & -5.448261000 & -1.132749000 & 1.090928000 \\ 6 & -4.087510000 & 0.533258000 & -0.664585000 \\ 1 & -3.591402000 & 0.601257000 & -1.636136000 \\ 1 & -5.163960000 & 0.427826000 & -0.846718000 \\ 1 & -3.929435000 & 1.471140000 & -0.124401000 \\ 8 & -3.638376000 & -1.850195000 & -0.655642000 \\ 1 & -4.559238000 & -1.962619000 & -0.920150000 \\ 6 & 1.649725000 & 3.351357000 & -0.694669000 \\ 1 & 2.597080000 & 3.354448000 & -0.141295000 \\ 1 & 1.905095000 & 3.085006000 & -1.734007000 \\ 8 & 1.145439000 & 4.665687000 & -0.614894000\end{array}$




$\begin{array}{rrrr}1 & 0.345939000 & 4.698942000 & -1.153734000 \\ 1 & -0.482607000 & 3.693403000 & 0.761147000 \\ 1 & -1.462624000 & 2.392118000 & 2.374262000 \\ 1 & 2.687806000 & 0.429879000 & -1.839948000 \\ 1 & -1.816530000 & -1.446675000 & 1.050540000 \\ 6 & 0.290115000 & -0.174112000 & -0.357852000 \\ 6 & -1.116039000 & -0.502414000 & -0.718886000 \\ 6 & -0.887062000 & -1.836634000 & -1.499978000 \\ 6 & 0.586434000 & -1.811258000 & -2.002085000 \\ 6 & 1.333790000 & -1.134786000 & -0.852602000 \\ 1 & -1.018016000 & -2.677251000 & -0.809911000 \\ 1 & -1.609988000 & -1.974661000 & -2.305422000 \\ 1 & 0.670098000 & -1.201375000 & -2.909985000 \\ 1 & 0.975174000 & -2.808643000 & -2.225744000 \\ 1 & -2.392096000 & 0.435370000 & 2.394521000 \\ 1 & -1.460703000 & 0.281316000 & -1.408832000\end{array}$

$\mathrm{TS}^{2}$

$\mathrm{C} 19 \mathrm{H} 26 \mathrm{O} 4$

C1

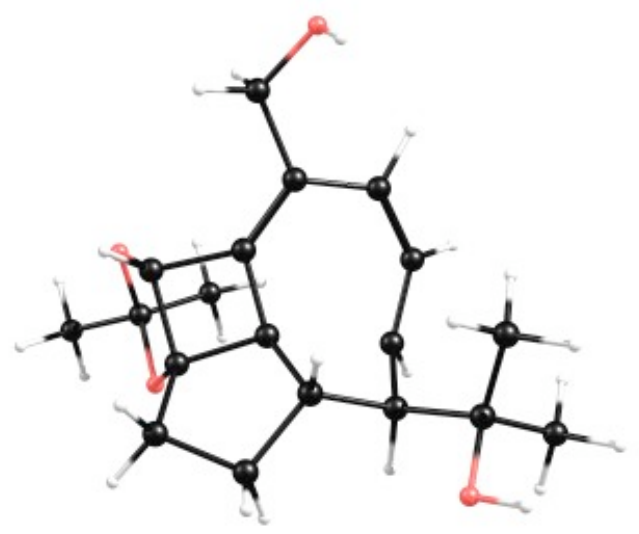

$\begin{array}{rrrr}6 & 2.070047000 & 0.124314000 & -1.050668000 \\ 6 & 0.810874000 & 0.825779000 & -0.546476000 \\ 8 & 2.237975000 & -1.829134000 & 0.240539000 \\ 8 & 3.253058000 & 0.165497000 & -0.288819000 \\ 6 & 3.178552000 & -0.859353000 & 0.711336000 \\ 6 & 4.539052000 & -1.528071000 & 0.798373000 \\ 1 & 5.295598000 & -0.805076000 & 1.114078000 \\ 1 & 4.511336000 & -2.346685000 & 1.522183000 \\ 1 & 4.812414000 & -1.926052000 & -0.181066000 \\ 6 & 2.728247000 & -0.272612000 & 2.045800000 \\ 1 & 3.445024000 & 0.482923000 & 2.380277000 \\ 1 & 1.744528000 & 0.190888000 & 1.953500000 \\ 1 & 2.672705000 & -1.062464000 & 2.800605000 \\ 6 & 0.383234000 & 2.116552000 & -0.300895000 \\ 6 & -2.025380000 & -0.917127000 & 0.396840000 \\ 6 & -1.229097000 & -0.065702000 & 1.398361000 \\ 6 & -0.606851000 & 2.320150000 & 0.681971000 \\ 6 & -1.125821000 & 1.339012000 & 1.526781000 \\ 6 & -3.520427000 & -0.580644000 & 0.206930000 \\ 6 & -4.256033000 & -0.647929000 & 1.549076000 \\ 1 & -4.112117000 & -1.627377000 & 2.015452000 \\ 1 & -3.905034000 & 0.124998000 & 2.239672000 \\ 1 & -5.332364000 & -0.497315000 & 1.402811000 \\ 6 & -3.782437000 & 0.764004000 & -0.472624000 \\ 1 & -3.333978000 & 0.795383000 & -1.468878000 \\ 1 & -4.862995000 & 0.917696000 & -0.586201000 \\ 1 & -3.386432000 & 1.596762000 & 0.113332000 \\ 8 & -3.973711000 & -1.642171000 & -0.642822000 \\ 1 & -4.900148000 & -1.469843000 & -0.849708000 \\ 6 & 1.160686000 & 3.316539000 & -0.809766000 \\ 1 & 2.008190000 & 3.521068000 & -0.145324000 \\ 1 & 1.586524000 & 3.085948000 & -1.798304000 \\ 8 & 0.402236000 & 4.509797000 & -0.838861000 \\ 1 & -0.358185000 & 4.351475000 & -1.410900000\end{array}$




$\begin{array}{rrr}-0.746306000 & 3.356075000 & 0.986484000 \\ -1.323547000 & 1.712855000 & 2.535654000 \\ 2.314820000 & 0.293637000 & -2.105514000 \\ -2.032728000 & -1.930684000 & 0.816724000 \\ 0.159610000 & -0.389094000 & -0.257482000 \\ -1.128159000 & -0.950247000 & -0.832393000 \\ -0.691154000 & -2.329256000 & -1.376661000 \\ 0.806368000 & -2.189769000 & -1.736881000 \\ 1.376486000 & -1.224426000 & -0.692164000 \\ -0.797312000 & -3.077576000 & -0.581897000 \\ -1.304314000 & -2.663395000 & -2.217890000 \\ 0.919816000 & -1.759174000 & -2.739158000 \\ 1.337785000 & -3.145310000 & -1.724802000 \\ -1.201193000 & -0.546260000 & 2.381015000 \\ -1.495333000 & -0.317127000 & -1.649190000\end{array}$

\section{f5} $\mathrm{C} 19 \mathrm{H} 26 \mathrm{O} 4$

C1

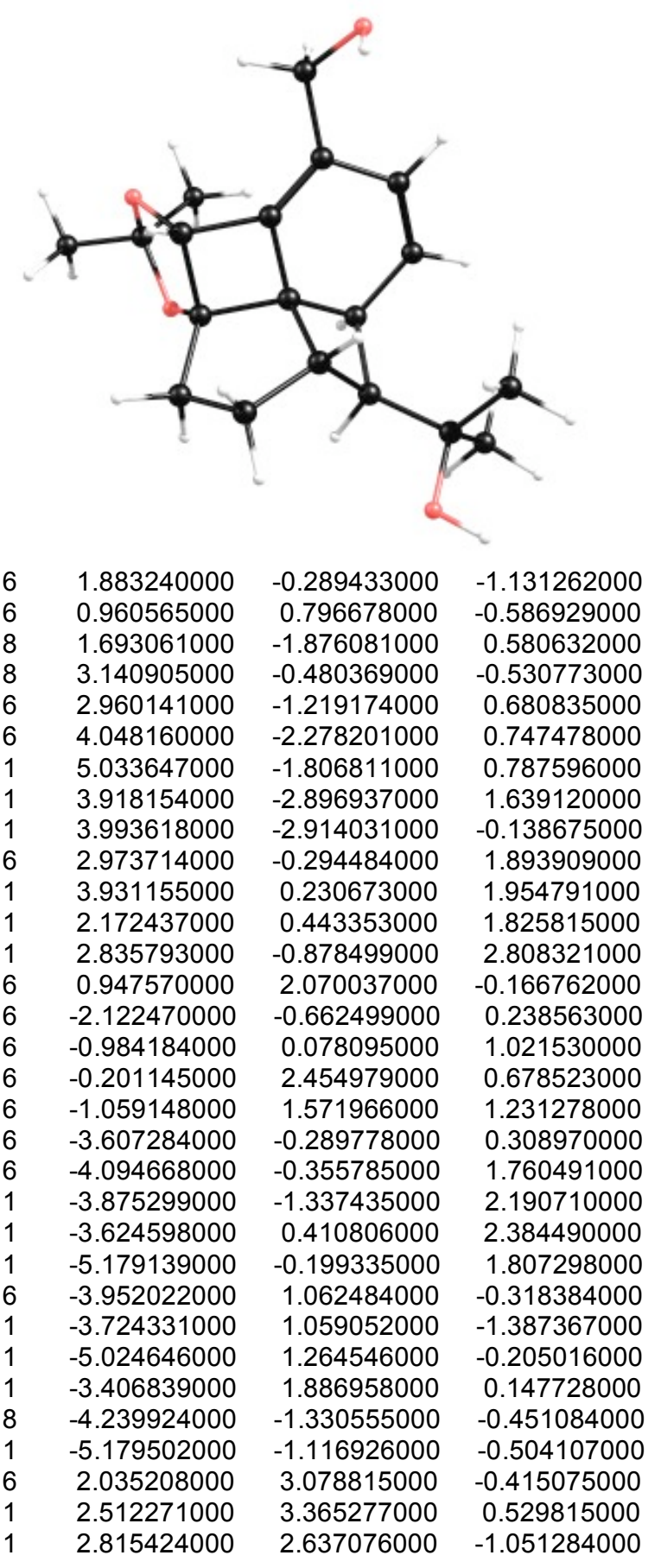


$-0.297988000$

3.512316000

0.914559000

$-1.800604000$

1.946822000

1.935594000

2.005321000

$-0.322942000$

$-2.219401000$

$-2.078965000$

$-1.717706000$

$-0.080526000$

$-0.184437000$

0.538403000

$-1.314218000$

$-0.491648000$

$-0.194111000$

$-0.987298000$

$-1.710661000$

077909000

0.192244000

$-2.422453000$

0.927469000

$-1.343585000$

$-1.856220000$

$-2.363399000$

$-0.689261000$

$-1.373530000$

0.848242000

$-2.948328000$

$-0.161340000$

$-3.165424000$

$-0.720707000$

$-0.409462000$

$-1.951528000$

$-1.263155000$

$-0.471907000$

$-2.079908000$

$-2.951886000$

$-1.964698000$

$-0.538323000$

1.971507000

$-1.659082000$

0.365600000

$-1.662681000$

\section{$X=8 \quad(r, r)$}

\section{t8}

\section{$\mathrm{C} 22 \mathrm{H} 32 \mathrm{O} 4$}

C1

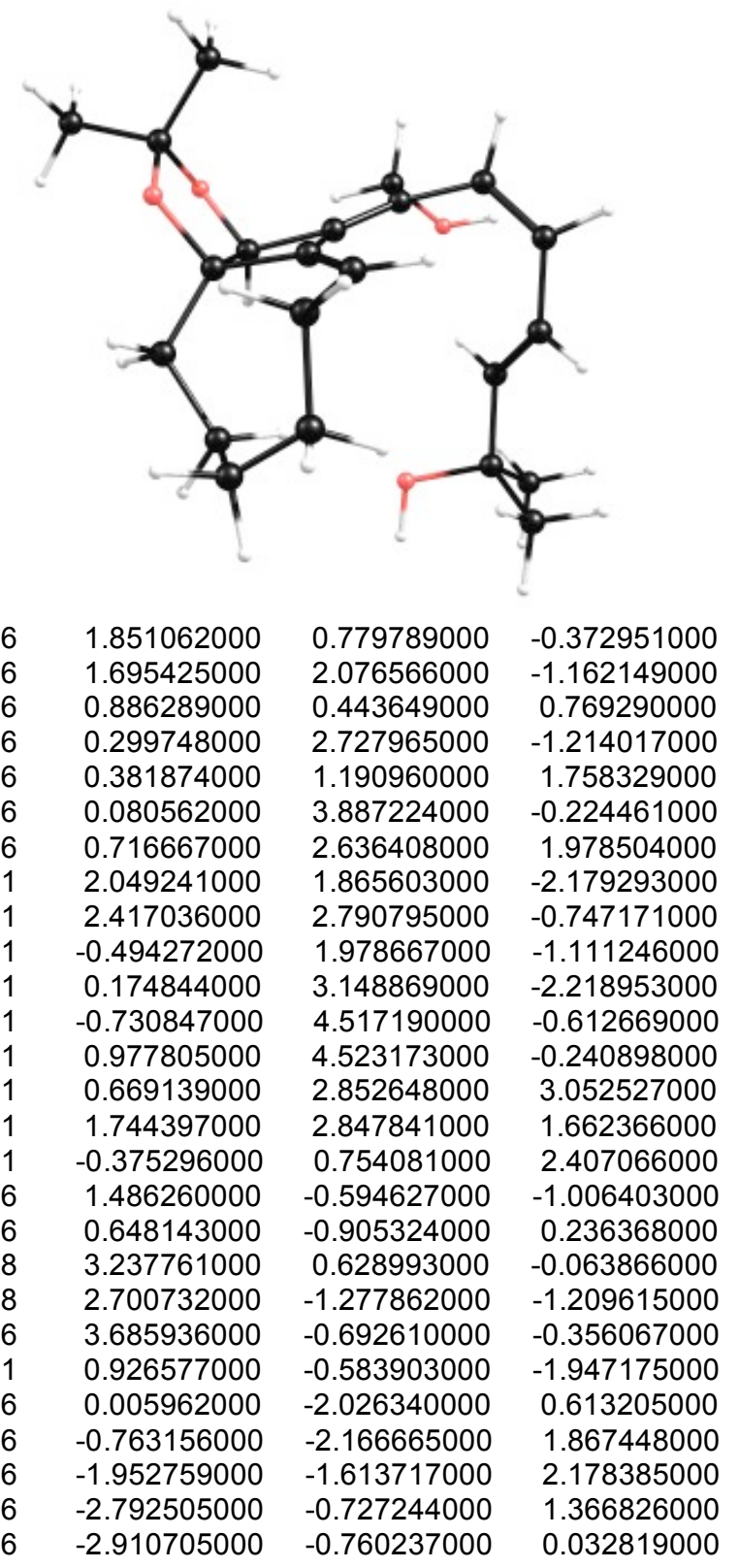


$-0.217651000$

0.605368000

$-4.071397000$

$-1.121842000$

$-1.191008000$

$-3.743246000$

$-1.735061000$

$-3.822878000$

0.361917000

3.845585000

$-1.497641000$

0.639571000

4.167345000

$-2.517417000$

0.154839000

0.931983000

4.597701000

$-1.028403000$

2.902047000

$-1.541191000$

0.701435000

4.985004000

$-0.599440000$

1.572651000

5.336351000

$-1.598416000$

4.818772000

$-0.019937000$

5.754504000

$-0.107774000$

$-3.788918000$

0.125679000

1.285730000

$-4.428200000$

1.907968000

1.912340000

$-5.003438000$

0.935155000

$-5.118937000$

$-0.740142000$

$-4.863827000$

$-1.545823000$

$-1.183081000$

$-0.134426000$

$-5.467650000$

0.637730000

$-2.896735000$

1.129136000

$-3.432187000$

$-2.850260000$

$-2.342796000$

$-1.827970000$

$-0.265625000$

3.569817000

$-0.322904000$

4.524936000

1.478264000

$-1.140360000$

$-1.412163000$

$-2.050764000$

$-0.539599000$

$-0.816694000$

$-0.054769000$

0.411225000

$-0.745832000$

0.718502000

$-1.492040000$

$-2.064764000$

$-0.754187000$

$-2.179810000$

$-1.817815000$

$-2.453094000$

2.602914000

3.174316000

1.235780000

1.773978000

$-1.271415000$

3.132739000

1.288455000

$\mathrm{TS}^{1}$

$\mathrm{C} 22 \mathrm{H} 32 \mathrm{O} 4$

C1

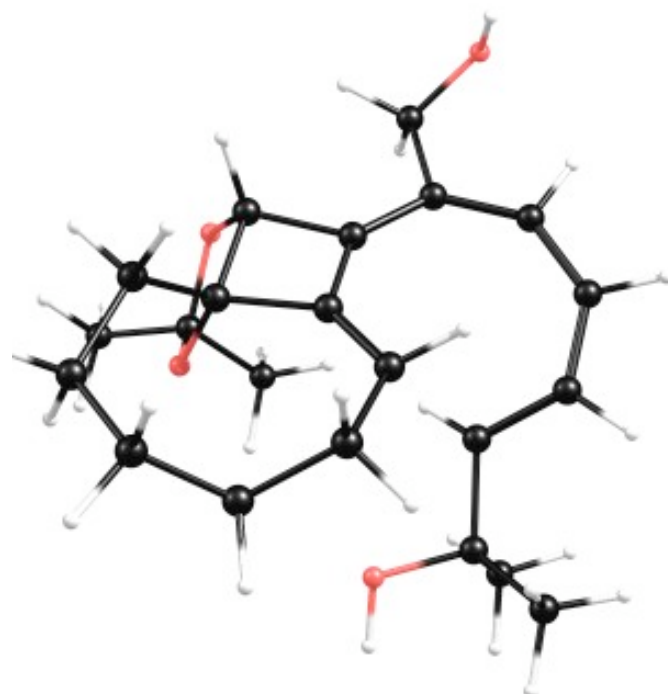

2.323938000

1.180495000

1.209497000

2.907368000

2.058605000

2.932053000

3.626886000

2.311756000

3.503049000

1.223219000

1.869314000

0.687784000

0.496724000

1.209455000
$-0.061312000$

0.913620000

$-1.683869000$

$-0.137970000$

$-0.926043000$

$-1.892594000$

$-1.342052000$

$-2.537549000$

$-2.513422000$

$-0.035116000$

0.482316000

0.714369000

$-0.639422000$

2.316160000
$-0.383467000$

$-0.650409000$

0.865452000

0.897681000

1.731590000

2.516554000

3.156491000

3.144748000

1.822927000

2.645828000

3.360943000

2.061113000

3.195634000

$-0.648700000$ 


$\begin{array}{rrrr}6 & -1.955583000 & 1.083935000 & 0.253930000 \\ 6 & -2.168847000 & 2.278515000 & -0.443168000 \\ 6 & 0.177969000 & 3.245182000 & -0.843596000 \\ 6 & -1.224071000 & 3.206168000 & -0.877523000 \\ 6 & -3.108384000 & 0.417555000 & 0.990887000 \\ 6 & -3.293994000 & 1.149079000 & 2.333925000 \\ 1 & -2.366519000 & 1.120163000 & 2.912163000 \\ 1 & -3.578587000 & 2.194272000 & 2.183948000 \\ 1 & -4.082328000 & 0.664014000 & 2.924653000 \\ 6 & -4.434950000 & 0.406364000 & 0.223411000 \\ 1 & -4.325579000 & -0.047828000 & -0.763632000 \\ 1 & -5.174928000 & -0.176310000 & 0.784047000 \\ 1 & -4.847654000 & 1.411286000 & 0.098352000 \\ 8 & -2.684811000 & -0.920045000 & 1.251350000 \\ 1 & -3.348814000 & -1.326739000 & 1.820873000 \\ 6 & 2.598576000 & 2.926833000 & -0.417696000 \\ 1 & 2.740701000 & 3.109474000 & 0.653397000 \\ 1 & 3.376836000 & 2.211263000 & -0.714099000 \\ 8 & 2.805460000 & 4.179051000 & -1.043903000 \\ 1 & 2.727343000 & 4.038625000 & -1.995180000 \\ 1 & 0.573318000 & 4.247521000 & -1.007761000 \\ 1 & -1.663560000 & 4.120317000 & -1.277523000 \\ 1 & 3.129391000 & -0.065513000 & -1.126758000 \\ 1 & -1.024793000 & 0.990622000 & 0.799603000 \\ 6 & 0.272747000 & -0.134523000 & -0.886056000 \\ 6 & -1.038265000 & -0.142079000 & -1.375852000 \\ 6 & -1.801714000 & -1.387054000 & -1.754155000 \\ 6 & -1.556480000 & -2.698008000 & -0.985345000 \\ 6 & 1.620853000 & -2.369548000 & -1.415908000 \\ 6 & 1.292086000 & -1.212254000 & -0.475388000 \\ 1 & -2.865986000 & -1.138824000 & -1.707495000 \\ 1 & -1.611721000 & -1.556234000 & -2.827245000 \\ 1 & -1.220191000 & -2.470810000 & 0.030065000 \\ 1 & -2.531332000 & -3.183702000 & -0.867350000 \\ 1 & 1.506775000 & -2.022488000 & -2.451758000 \\ 1 & 2.688480000 & -2.585185000 & -1.275617000 \\ 1 & -3.196502000 & 2.516599000 & -0.715007000 \\ 1 & -1.276715000 & 0.703939000 & -2.009242000 \\ 6 & -0.605425000 & -3.702464000 & -1.660167000 \\ 1 & -0.646019000 & -3.573556000 & -2.751967000 \\ 1 & -0.979058000 & -4.717391000 & -1.473422000 \\ 6 & 0.850099000 & -3.672073000 & -1.195984000 \\ 1 & 1.391129000 & -4.472254000 & -1.717680000 \\ 1 & 0.896408000 & -3.914879000 & -0.128131000\end{array}$

c8

$\mathrm{C} 22 \mathrm{H} 32 \mathrm{O} 4$

C1

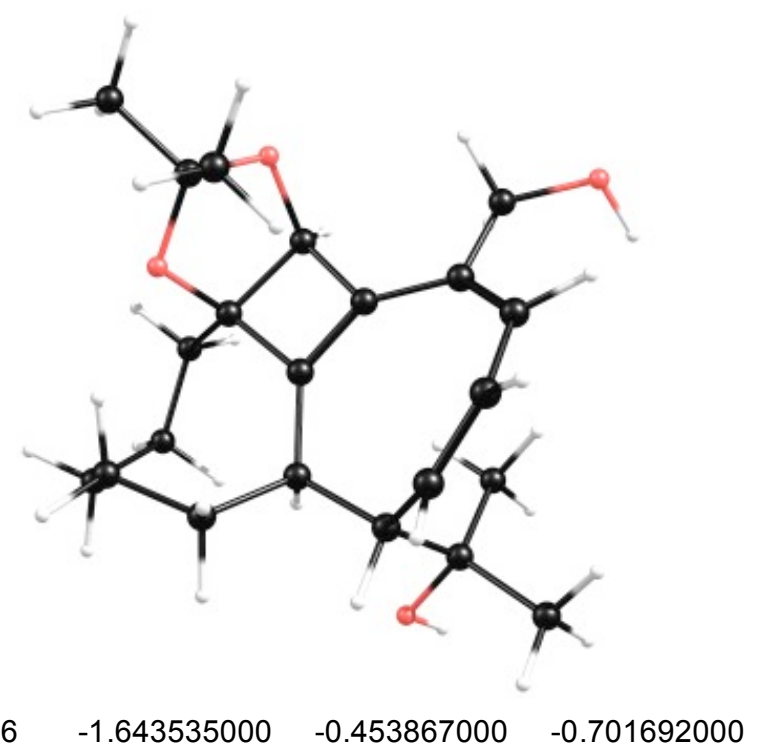




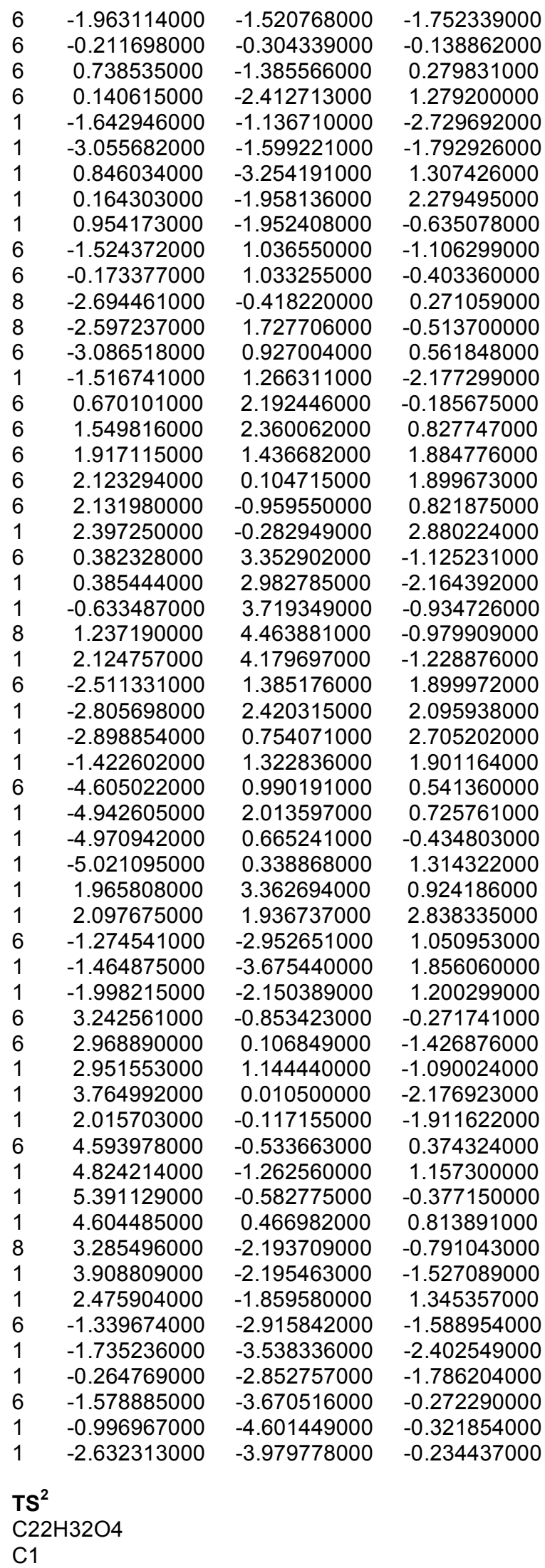




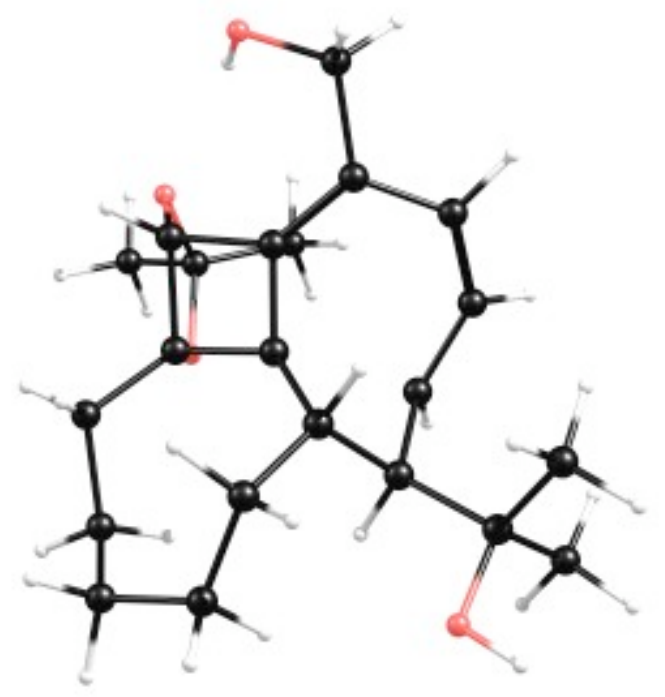

$6-1.092737000$

$8-1.666374000$

$8-3.272724000$

$6-2.889351000$

$6-3.963726000$

$1-4.920316000$

$1-3.683514000$

$1-4.078545000$

$6-2.688783000$

$1-3.628749000$

$1-1.930578000$

$1-2.362306000$

$6-1.106180000$

$6 \quad 2.013205000$

$6 \quad 0.876991000$

$6-0.272843000$

$6 \quad 0.415501000$

$6 \quad 3.406198000$

$6 \quad 3.766185000$

13.696080000

13.111822000

14.795750000

$6 \quad 3.532821000$

13.395886000

14.531944000

12.800307000

84.299472000

15.187080000

$6-2.208774000$

$1-1.834691000$

$1-2.471380000$

$8-3.344823000$

$1-3.619122000$

$1-0.452504000$

$1 \quad 0.408483000$

$1-2.454347000$

$1 \quad 2.130829000$

$6-0.088569000$

$6 \quad 1.371903000$

$6 \quad 1.975061000$

$6 \quad 2.479620000$

$6-0.842024000$

$6-1.114730000$

$1 \quad 2.847257000$

$1 \quad 1.279308000$

12.926963000

13.321888000

$1-0.600685000$
0.360592000

$-0.735221000$

1.334398000

0.236972000

0.599534000

1.518909000

0.993108000

1.858834000

2.387833000

$-0.645869000$

$-1.199593000$

$-1.294918000$

$-0.359074000$

$-2.115046000$

$-0.284643000$

$-1.036848000$

$-2.863878000$

$-2.346320000$

$-0.957471000$

$-1.337663000$

$-0.464064000$

$-2.122624000$

$-1.712980000$

$-2.170873000$

$-1.882644000$

$-2.613736000$

$-2.944067000$

0.072346000

$-0.305369000$

$-2.830127000$

$-3.129053000$

$-3.762428000$

$-2.033772000$

$-1.629581000$

$-3.939036000$

$-3.020312000$

0.707210000

0.650206000

0.096356000

0.053878000

1.196153000

2.477116000

2.643182000

1.250119000

0.749421000

1.446616000

2.215055000

2.846412000

2.571861000
$-0.910337000$ $-0.888797000$

1.157799000

$-0.079152000$

1.256115000

1.815522000

1.878438000

2.815990000

1.164183000

2.110642000

2.196028000

1.669007000

3.114345000

$-1.002618000$

0.528469000

1.224634000

$-0.143703000$

0.954552000

0.575389000

2.015337000

2.670861000

2.405559000

2.059866000

$-0.345934000$

$-1.391568000$

$-0.247723000$

$-0.099881000$

0.129994000

0.110204000

$-1.761036000$

$-2.749418000$

$-1.233702000$

$-2.006186000$

$-1.169860000$

$-0.126953000$

1.816282000

$-1.896312000$

1.089881000

$-0.348893000$

$-0.805353000$

$-1.649996000$

$-0.958764000$

$-0.677823000$

$-0.151652000$

$-2.139260000$

$-2.462265000$

0.005701000

$-1.557404000$

$-1.745245000$ 


\section{f8}

\section{$\mathrm{C} 22 \mathrm{H} 32 \mathrm{O} 4$}

C1

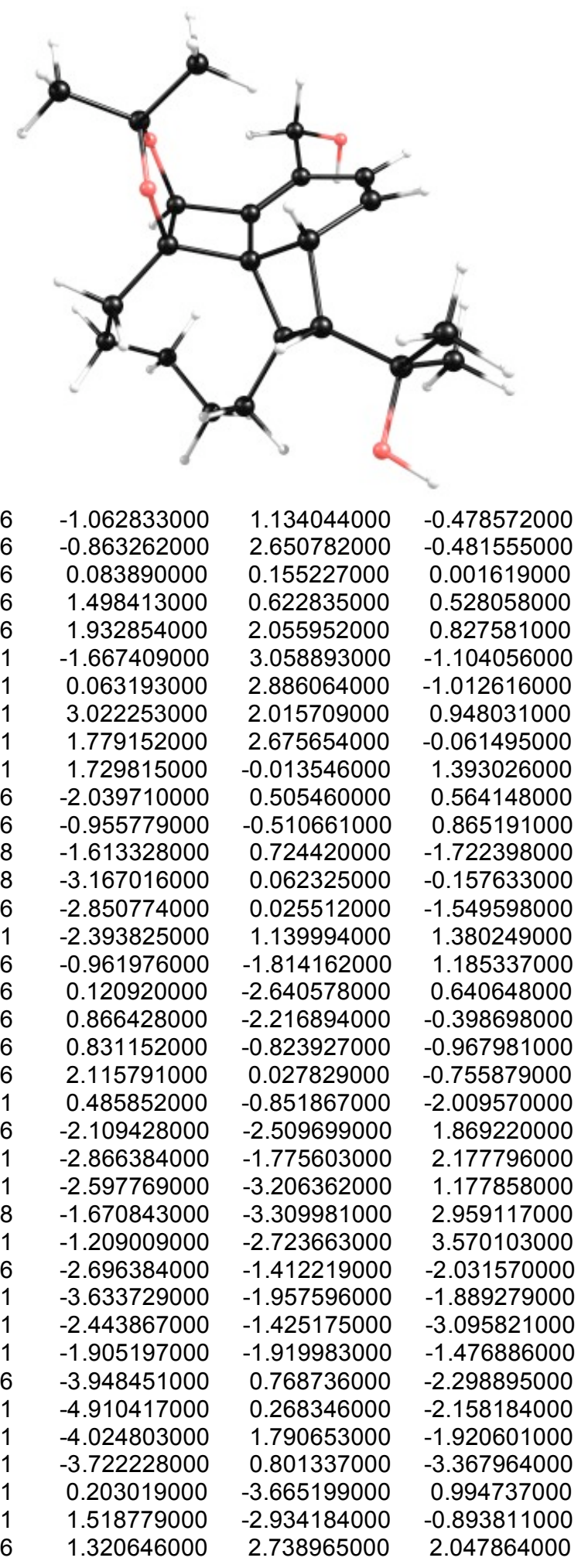


$-2.196369000$

5.283978000

2.098076000 0.655145000 0.365800000 0.813939000

$-0.928220000 \quad 3.359933000$ $-0.569715000 \quad 4.389203000$ $-1.987446000$

$-2.183530000$

$-0.785137000$

$-0.752810000$

$-1.522638000$

0.886911000

0.751095000

1.158169000

2.091252000

2.248282000

$-0.525698000$

\section{$X=8 \quad(r, s)$}

\section{t8}

\section{$\mathrm{C} 22 \mathrm{H} 32 \mathrm{O} 4$}

C1

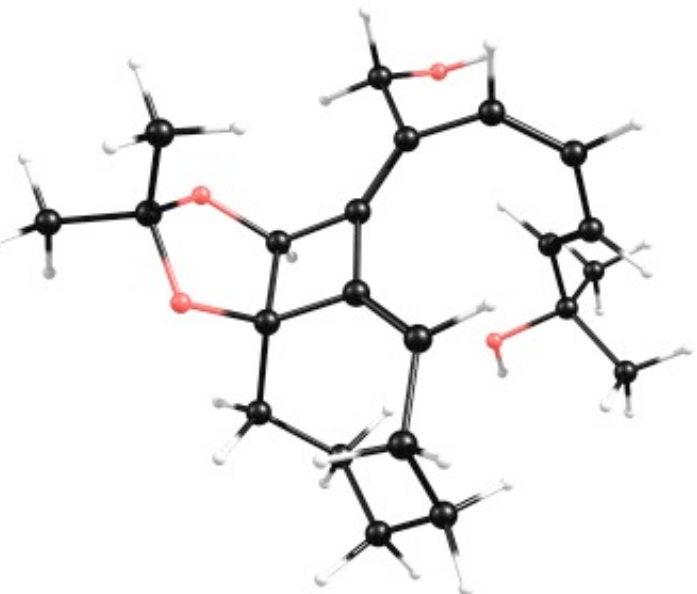

$\begin{array}{rrrr}6 & 1.851062000 & 0.779789000 & -0.372951000 \\ 6 & 1.695425000 & 2.076566000 & -1.162149000 \\ 6 & 0.886289000 & 0.443649000 & 0.769290000 \\ 6 & 0.299748000 & 2.727965000 & -1.214017000 \\ 6 & 0.381874000 & 1.190960000 & 1.758329000 \\ 6 & 0.080562000 & 3.887224000 & -0.224461000 \\ 6 & 0.716667000 & 2.636408000 & 1.978504000 \\ 1 & 2.049241000 & 1.865603000 & -2.179293000 \\ 1 & 2.417036000 & 2.790795000 & -0.747171000 \\ 1 & -0.494272000 & 1.978667000 & -1.111246000 \\ 1 & 0.174844000 & 3.148869000 & -2.218953000 \\ 1 & -0.730847000 & 4.517190000 & -0.612669000 \\ 1 & 0.977805000 & 4.523173000 & -0.240898000 \\ 1 & 0.669139000 & 2.852648000 & 3.052527000 \\ 1 & 1.744397000 & 2.847841000 & 1.662366000 \\ 1 & -0.375296000 & 0.754081000 & 2.407066000 \\ 6 & 1.486260000 & -0.594627000 & -1.006403000 \\ 6 & 0.648143000 & -0.905324000 & 0.236368000 \\ 8 & 3.237761000 & 0.628993000 & -0.063866000 \\ 8 & 2.700732000 & -1.277862000 & -1.209615000 \\ 6 & 3.685936000 & -0.692610000 & -0.356067000 \\ 1 & 0.926577000 & -0.583903000 & -1.947175000 \\ 6 & 0.005962000 & -2.026340000 & 0.613205000 \\ 6 & -0.763156000 & -2.166665000 & 1.867448000 \\ 6 & -1.952759000 & -1.613717000 & 2.178385000\end{array}$




$\begin{array}{rrrr}6 & -2.792505000 & -0.727244000 & 1.366826000 \\ 6 & -2.910705000 & -0.760237000 & 0.032819000 \\ 1 & -3.393578000 & -0.019091000 & 1.936832000 \\ 1 & -2.353385000 & -1.500560000 & -0.539139000 \\ 6 & 0.088148000 & -3.286280000 & -0.217651000 \\ 1 & 0.675153000 & -3.114645000 & -1.121842000 \\ 1 & 0.605368000 & -4.071397000 & 0.361917000 \\ 8 & -1.191008000 & -3.743246000 & -0.639571000 \\ 1 & -1.735061000 & -3.822878000 & 0.154839000 \\ 6 & 3.845585000 & -1.497641000 & 0.931983000 \\ 1 & 4.167345000 & -2.517417000 & 0.701435000 \\ 1 & 4.597701000 & -1.028403000 & 1.572651000 \\ 1 & 2.902047000 & -1.541191000 & 1.478264000 \\ 6 & 4.985004000 & -0.599440000 & -1.140360000 \\ 1 & 5.336351000 & -1.598416000 & -1.412163000 \\ 1 & 4.818772000 & -0.019937000 & -2.050764000 \\ 1 & 5.754504000 & -0.107774000 & -0.539599000 \\ 6 & -3.788918000 & 0.125679000 & -0.816694000 \\ 6 & -4.428200000 & 1.285730000 & -0.054769000 \\ 1 & -3.660083000 & 1.907968000 & 0.411225000 \\ 1 & -5.003438000 & 1.912340000 & -0.745832000 \\ 1 & -5.118937000 & 0.935155000 & 0.718502000 \\ 6 & -4.863827000 & -0.740142000 & -1.492040000 \\ 1 & -4.396249000 & -1.545823000 & -2.064764000 \\ 1 & -5.539565000 & -1.183081000 & -0.754187000 \\ 1 & -5.467650000 & -0.134426000 & -2.179810000 \\ 8 & -2.896735000 & 0.637730000 & -1.817815000 \\ 1 & -3.432187000 & 1.129136000 & -2.453094000 \\ 1 & -0.330444000 & -2.850260000 & 2.602914000 \\ 1 & -2.342796000 & -1.827970000 & 3.174316000 \\ 6 & -0.265625000 & 3.569817000 & 1.235780000 \\ 1 & -0.322904000 & 4.524936000 & 1.773978000 \\ 1 & -1.271415000 & 3.132739000 & 1.288455000\end{array}$

TS $^{1}$

$\mathrm{C} 22 \mathrm{H} 32 \mathrm{O} 4$

C1

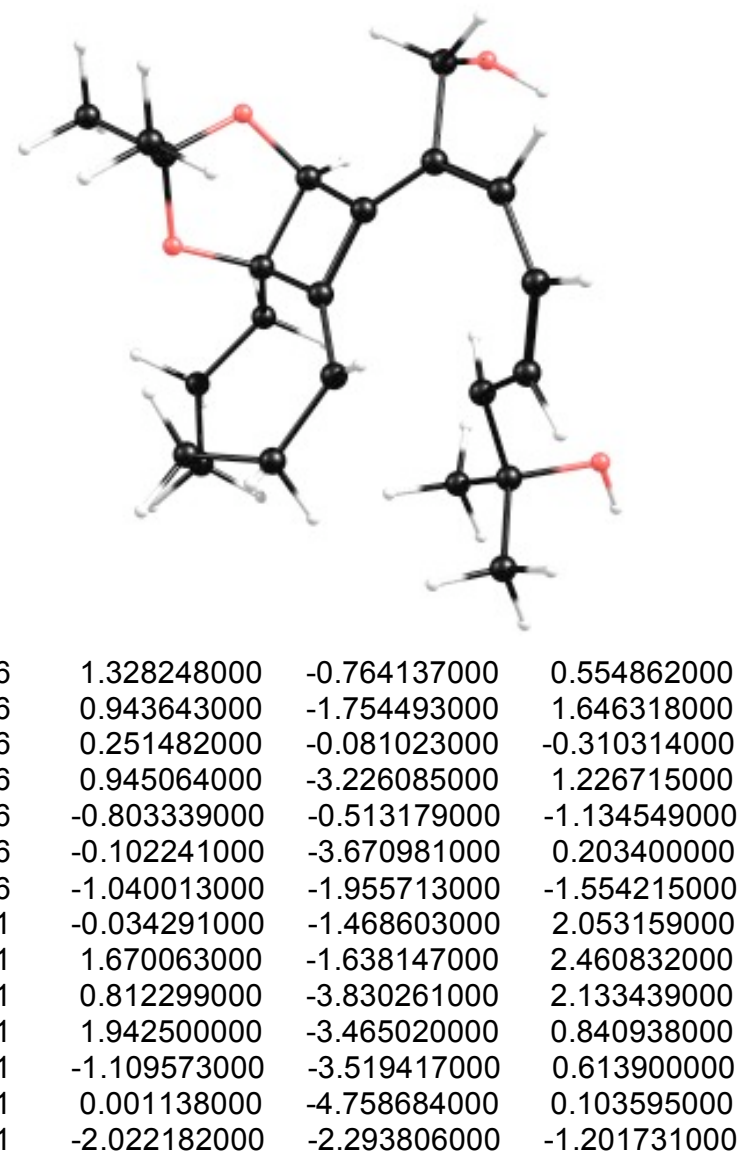




$\begin{array}{rrrr}1 & -1.138985000 & -1.933962000 & -2.647267000 \\ 1 & -1.032731000 & 0.188595000 & -1.924231000 \\ 6 & 1.782770000 & 0.663910000 & 0.954809000 \\ 6 & 0.679470000 & 1.203503000 & 0.051020000 \\ 8 & 2.445082000 & -1.277080000 & -0.169786000 \\ 8 & 3.128795000 & 0.783928000 & 0.542174000 \\ 6 & 3.408338000 & -0.246712000 & -0.401497000 \\ 1 & 1.721614000 & 0.959900000 & 2.005877000 \\ 6 & 0.353307000 & 2.550785000 & -0.193776000 \\ 6 & -0.714250000 & 3.109173000 & -0.905676000 \\ 6 & -1.961988000 & 2.650582000 & -1.370117000 \\ 6 & -2.736028000 & 1.545735000 & -1.042904000 \\ 6 & -2.459765000 & 0.553659000 & -0.087841000 \\ 1 & -3.655778000 & 1.440745000 & -1.614681000 \\ 1 & -1.760535000 & 0.830140000 & 0.693834000 \\ 6 & 1.328248000 & 3.550544000 & 0.417514000 \\ 1 & 2.355919000 & 3.186481000 & 0.350816000 \\ 1 & 1.270664000 & 4.505134000 & -0.128115000 \\ 8 & 1.083042000 & 3.736765000 & 1.806410000 \\ 1 & 0.151837000 & 3.973624000 & 1.896194000 \\ 6 & 3.311028000 & 0.273774000 & -1.834296000 \\ 1 & 4.046027000 & 1.068346000 & -1.992196000 \\ 1 & 3.515807000 & -0.536198000 & -2.540527000 \\ 1 & 2.315963000 & 0.672105000 & -2.036617000 \\ 6 & 4.786822000 & -0.806932000 & -0.090175000 \\ 1 & 5.545576000 & -0.029482000 & -0.212132000 \\ 1 & 4.809758000 & -1.166733000 & 0.940483000 \\ 1 & 5.021318000 & -1.634928000 & -0.764336000 \\ 6 & -3.624510000 & -0.286228000 & 0.440458000 \\ 6 & -3.141423000 & -1.323626000 & 1.452257000 \\ 1 & -2.498919000 & -2.076729000 & 0.992327000 \\ 1 & -2.584094000 & -0.832077000 & 2.255124000 \\ 1 & -3.995171000 & -1.846453000 & 1.900575000 \\ 6 & -4.479528000 & -0.937618000 & -0.650265000 \\ 1 & -3.910295000 & -1.636293000 & -1.268811000 \\ 1 & -5.300170000 & -1.498283000 & -0.187797000 \\ 1 & -4.925869000 & -0.182571000 & -1.301433000 \\ 8 & -4.429514000 & 0.685641000 & 1.135392000 \\ 1 & -5.115633000 & 0.195937000 & 1.607973000 \\ 1 & -0.549577000 & 4.161438000 & -1.146852000 \\ 1 & -2.439838000 & 3.339137000 & -2.066606000 \\ 6 & -0.012812000 & -3.047754000 & -1.205634000 \\ 1 & -0.183180000 & -3.849344000 & -1.934264000 \\ 1 & 1.001970000 & -2.681719000 & -1.391562000\end{array}$

c8 $\mathrm{C} 22 \mathrm{H} 32 \mathrm{O} 4$

C1

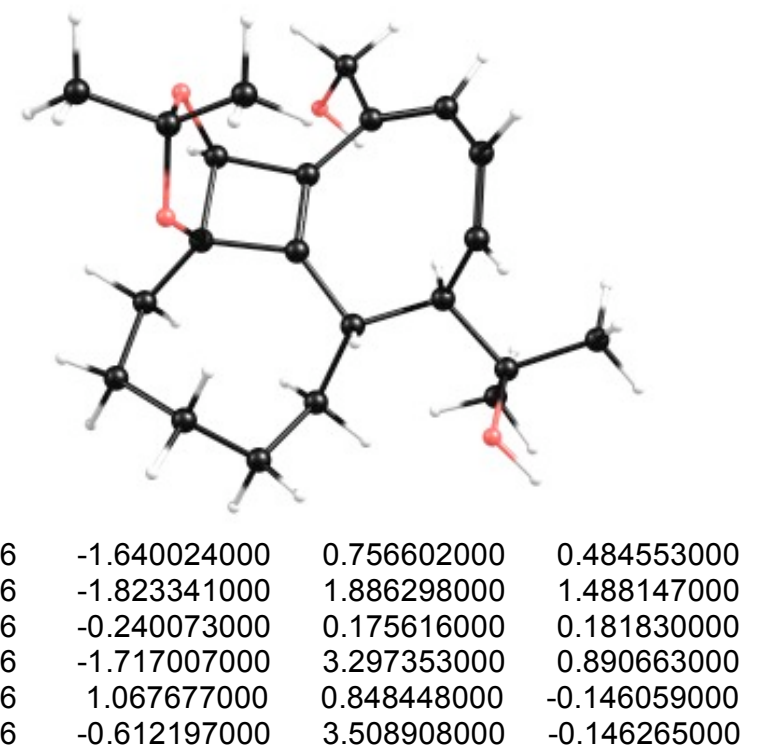




\begin{tabular}{|c|c|c|c|}
\hline 3 & 1.337123000 & 2.022344000 & 0.831643000 \\
\hline 1 & -1.103517000 & 1.743641000 & 2.301330000 \\
\hline 1 & -2.818419000 & 1.771723000 & 1.936456000 \\
\hline 1 & -1.604000000 & 4.020771000 & 1.709405000 \\
\hline 1 & -2.671177000 & 3.527542000 & 0.404305000 \\
\hline 1 & -0.735419000 & 4.516877000 & -0.563942000 \\
\hline 1 & -0.785491000 & 2.824138000 & -0.984689000 \\
\hline 1 & 0.944017000 & 1.782130000 & 1.825886000 \\
\hline 1 & 2.412782000 & 2.144346000 & 0.940043000 \\
\hline 1 & 0.988700000 & 1.302807000 & -1.143160000 \\
\hline 6 & -2.030954000 & -0.681923000 & 0.894056000 \\
\hline 6 & -0.629022000 & -1.097941000 & 0.472220000 \\
\hline 8 & -2.450878000 & 1.012228000 & -0.659442000 \\
\hline 8 & -3.117763000 & -1.053699000 & 0.074469000 \\
\hline 6 & -3.155194000 & -0.171395000 & -1.046500000 \\
\hline 1 & -2.296386000 & -0.871487000 & 1.937958000 \\
\hline 6 & -0.135888000 & -2.469855000 & 0.421246000 \\
\hline 6 & 0.681785000 & -2.974115000 & -0.536067000 \\
\hline 6 & 1.279356000 & -2.229527000 & -1.631831000 \\
\hline 6 & 1.864052000 & -1.023735000 & -1.538349000 \\
\hline 6 & 2.156718000 & -0.271687000 & -0.270772000 \\
\hline 1 & 2.199650000 & -0.541053000 & -2.455880000 \\
\hline 1 & 2.026731000 & -0.961318000 & 0.571654000 \\
\hline 6 & -0.771167000 & -3.404367000 & 1.427299000 \\
\hline 1 & -1.854335000 & -3.439308000 & 1.263420000 \\
\hline 1 & -0.383735000 & -4.424689000 & 1.284028000 \\
\hline 8 & -0.610254000 & -2.967907000 & 2.768605000 \\
\hline 1 & 0.335990000 & -2.861196000 & 2.920559000 \\
\hline 6 & -2.504704000 & -0.807716000 & -2.272727000 \\
\hline 1 & -3.037913000 & -1.723595000 & -2.543976000 \\
\hline 1 & -2.549133000 & -0.114389000 & -3.117958000 \\
\hline 1 & -1.459771000 & -1.052044000 & -2.076 \\
\hline 6 & -4.606958000 & 0.202822000 & 377000 \\
\hline 1 & -5.185922000 & -0.686325000 & -1.564257000 \\
\hline 1 & -5.031312000 & 0.648744000 & -0.398421000 \\
\hline 1 & -4.674482000 & 0.922229000 & -2.120727000 \\
\hline 6 & 3.652665000 & 0.178960000 & -0.224435000 \\
\hline 6 & 4.574251000 & -0.826223000 & -0.933301000 \\
\hline 1 & 4.386766000 & -0.854466000 & -2.008375000 \\
\hline 1 & 5.621801000 & -0.537938000 & -0.778125000 \\
\hline 1 & 4.447323000 & -1.838332000 & -0.537834000 \\
\hline 6 & 4.154161000 & 0.297589000 & 1.226110000 \\
\hline 1 & 3.505509000 & 0.901817000 & 1.860395000 \\
\hline 1 & 4.234432000 & -0.695970000 & 1.679507000 \\
\hline 1 & 5.155295000 & 0.745117000 & 1.240002000 \\
\hline 8 & 3.719235000 & 1.432798000 & -0.907943000 \\
\hline 1 & 4.649605000 & 1.679351000 & -0.973657000 \\
\hline 1 & 0.830403000 & -4.055287000 & -0.544159000 \\
\hline 1 & 1.241042000 & -2.709134000 & -2.610738000 \\
\hline 6 & 0.831615000 & 3.389388000 & 0.342454000 \\
\hline 1 & 1.489145000 & 3.691559000 & -0.483812000 \\
\hline 1 & 1.001858000 & 4.121319000 & 1.144699000 \\
\hline \multicolumn{4}{|c|}{$\mathrm{TS}^{2}$} \\
\hline & & & \\
\hline
\end{tabular}




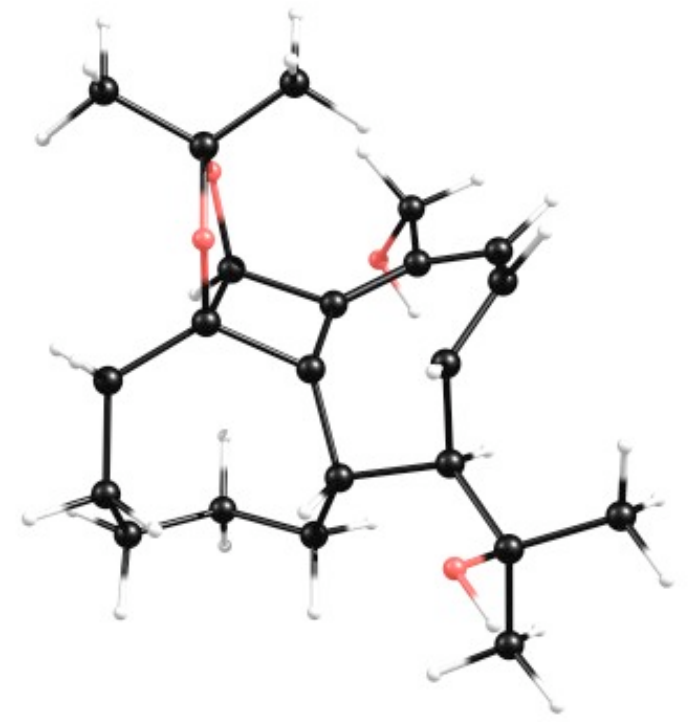

\begin{tabular}{rrrr}
6 & -1.256595000 & 1.045129000 & -0.534114000 \\
6 & -1.330254000 & 2.569433000 & -0.739626000 \\
6 & 0.020887000 & 0.256522000 & -0.127654000 \\
6 & -0.152707000 & 3.483095000 & -0.375923000 \\
6 & 1.433206000 & 0.794112000 & 0.073519000 \\
6 & 0.184143000 & 3.677962000 & 1.105139000 \\
6 & 1.674202000 & 1.554357000 & 1.393939000 \\
1 & -2.238020000 & 2.929397000 & -0.234712000 \\
1 & -1.516975000 & 2.699509000 & -1.810695000 \\
1 & -0.398034000 & 4.473118000 & -0.782454000 \\
1 & 0.739566000 & 3.168328000 & -0.925811000 \\
1 & -0.662612000 & 4.177644000 & 1.596370000 \\
1 & 1.022971000 & 4.385339000 & 1.165337000 \\
1 & 1.893529000 & 0.806848000 & 2.168741000 \\
1 & 2.579278000 & 2.167607000 & 1.298603000 \\
1 & 1.682838000 & 1.421396000 & -0.786885000 \\
6 & -1.965929000 & 0.341785000 & 0.662803000 \\
6 & -0.731756000 & -0.540573000 & 0.776354000 \\
8 & -1.914680000 & 0.431672000 & -1.646106000 \\
8 & -3.163917000 & -0.210366000 & 0.177276000 \\
6 & -3.077904000 & -0.295741000 & -1.247223000 \\
1 & -2.196673000 & 0.965498000 & 1.530236000 \\
6 & -0.614640000 & -1.859549000 & 1.207751000 \\
6 & 0.083163000 & -2.806299000 & 0.427799000 \\
6 & 0.688485000 & -2.488977000 & -0.785152000 \\
6 & 1.201443000 & -1.213541000 & -1.071534000 \\
6 & 2.176528000 & -0.527979000 & -0.119785000 \\
1 & 1.378025000 & -0.964741000 & -2.117901000 \\
1 & 2.193372000 & -1.083584000 & 0.826529000 \\
6 & -1.567505000 & -2.344220000 & 2.279839000 \\
1 & -2.599740000 & -2.235445000 & 1.929081000 \\
1 & -1.393486000 & -3.414811000 & 2.473166000 \\
8 & -1.497134000 & -1.582699000 & 3.474886000 \\
1 & -0.573108000 & -1.575386000 & 3.750557000 \\
6 & -2.931456000 & -1.743674000 & -1.693196000 \\
1 & -3.801138000 & -2.326052000 & -1.375347000 \\
1 & -2.856295000 & -1.793510000 & -2.783294000 \\
1 & -2.029013000 & -2.178777000 & -1.260069000 \\
6 & -4.320280000 & 0.366593000 & -1.829814000 \\
1 & -5.220608000 & -0.163773000 & -1.507128000 \\
1 & -4.381187000 & 1.402336000 & -1.487815000 \\
1 & -4.277915000 & 0.355392000 & -2.922261000 \\
6 & 3.619916000 & -0.479844000 & -0.667970000 \\
6 & 4.136784000 & -1.894117000 & -0.951247000 \\
1 & 3.502383000 & -2.404735000 & -1.679242000 \\
6 & 5.156801000 & -1.851529000 & -1.352999000 \\
\hline & 4.165681000 & -2.498066000 & -0.038543000 \\
\hline & 4.571639000 & 0.232829000 & 0.298335000
\end{tabular}




\section{f8}

\section{$\mathrm{C} 22 \mathrm{H} 32 \mathrm{O} 4$}

C1

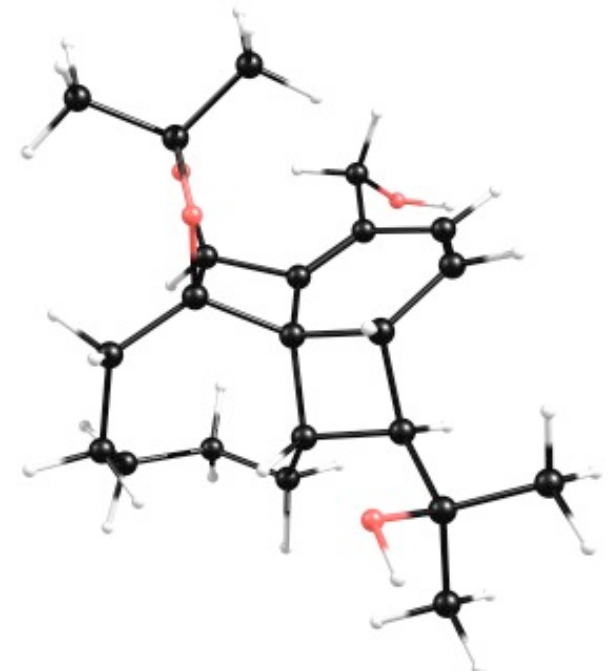

$\begin{array}{rrrr}6 & -0.875967000 & 1.136458000 & -0.480084000 \\ 6 & -0.577588000 & 2.637039000 & -0.595620000 \\ 6 & 0.189741000 & 0.063448000 & -0.053601000 \\ 6 & 0.421552000 & 3.372451000 & 0.320881000 \\ 6 & 1.556991000 & 0.557269000 & 0.498046000 \\ 6 & 0.357975000 & 3.227095000 & 1.850335000 \\ 6 & 1.735264000 & 0.975650000 & 1.954681000 \\ 1 & -1.555652000 & 3.129825000 & -0.508087000 \\ 1 & -0.255840000 & 2.808444000 & -1.629920000 \\ 1 & 0.272237000 & 4.439887000 & 0.109272000 \\ 1 & 1.444478000 & 3.173093000 & -0.009934000 \\ 1 & -0.613962000 & 3.595590000 & 2.208186000 \\ 1 & 1.097752000 & 3.936055000 & 2.246322000 \\ 1 & 1.784152000 & 0.075224000 & 2.581704000 \\ 1 & 2.703718000 & 1.481581000 & 2.070579000 \\ 1 & 1.868565000 & 1.363830000 & -0.167821000 \\ 6 & -1.829742000 & 0.638082000 & 0.644525000 \\ 6 & -0.898187000 & -0.556280000 & 0.787192000 \\ 8 & -1.573476000 & 0.726943000 & -1.653004000 \\ 8 & -3.087648000 & 0.419564000 & 0.050581000 \\ 6 & -2.934865000 & 0.402850000 & -1.372520000 \\ 1 & -1.969997000 & 1.299607000 & 1.502744000 \\ 6 & -1.171307000 & -1.869853000 & 0.893801000 \\ 6 & -0.355158000 & -2.815112000 & 0.125894000 \\ 6 & 0.505853000 & -2.399318000 & -0.823330000 \\ 6 & 0.969178000 & -0.984479000 & -0.935229000 \\ 6 & 2.236153000 & -0.711505000 & -0.059084000 \\ 1 & 1.129064000 & -0.665078000 & -1.969459000 \\ 1 & 2.323597000 & -1.486005000 & 0.712370000 \\ 6 & -2.407622000 & -2.396441000 & 1.580273000 \\ 1 & -3.066843000 & -1.572842000 & 1.859739000 \\ 1 & -2.970519000 & -3.048819000 & 0.891877000 \\ 8 & -2.100229000 & -3.084943000 & 2.787585000 \\ 1 & -1.502508000 & -3.808175000 & 2.566311000 \\ 6 & -3.224704000 & -0.984024000 & -1.929183000\end{array}$




$\begin{array}{rrr}-4.243286000 & -1.287785000 & -1.672167000 \\ -3.125710000 & -0.978594000 & -3.018436000 \\ -2.516660000 & -1.705318000 & -1.517903000 \\ -3.862776000 & 1.461829000 & -1.957729000 \\ -4.904576000 & 1.222241000 & -1.726738000 \\ -3.628994000 & 2.441259000 & -1.534421000 \\ -3.745378000 & 1.508945000 & -3.043733000 \\ 3.585548000 & -0.575707000 & -0.772403000 \\ 3.966549000 & -1.890528000 & -1.460428000 \\ 3.221418000 & -2.158979000 & -2.214204000 \\ 4.936407000 & -1.794715000 & -1.964119000 \\ 4.047056000 & -2.711732000 & -0.740971000 \\ 4.680580000 & -0.152110000 & 0.211894000 \\ 4.445716000 & 0.819245000 & 0.654244000 \\ 4.800261000 & -0.882305000 & 1.018723000 \\ 5.646224000 & -0.065535000 & -0.301710000 \\ 3.408545000 & 0.444608000 & -1.759905000 \\ 4.247070000 & 0.548250000 & -2.224517000 \\ -0.590844000 & -3.876427000 & 0.201865000 \\ 0.952434000 & -3.127776000 & -1.497681000 \\ 0.611876000 & 1.858695000 & 2.510640000 \\ 0.817994000 & 2.045681000 & 3.572508000 \\ -0.300987000 & 1.258892000 & 2.509621000\end{array}$

\section{References of Supporting Information}

1. J. Suffert, J. Org. Chem. 1989, 54, 509-510

2. (a) J. Suffert, B. Salem, P. Klotz, J. Am. Chem. Soc. 2001, 123, 12107-12108. (b) B. Salem, J. Suffert, Angew. Chem. Int. Ed. 2004, 43, 2826-2830. (c) C. Hulot, G. Blond, J. Suffert, J. Am. Chem. Soc. 2008, 130, 5046-5047.

3. H. X. Zhang, F. Guibé, G. Balavoine, J. Org. Chem. 1990, 55, 1857-1867

4. W. P. Gallagher, I. Terstiege, R. E. Maleczka, J. Am. Chem. Soc. 2001, 123, 14, 3194-3204

5. C. Morril, R. H. Grubbs, J. Org. Chem. 2003, 68, 15, 6031-6034

6. B. M. Trost, M. T. Rudd, Org. Lett. 2003, 5, 24, 4599-4602

7. C. Spino, V. G. Gund, C. Nadeau, J. Comb. Chem. 2005, 7, 345-352

8. R. N. Hanson, L. Franke, F. Murphy, Nuclear Medicine and Biology 1996, 23, 5, 585-588.

9. Frisch, M. J.; Trucks, G. W.; Schlegel, H. B.; Scuseria, G. E.; Robb,M. A.; Cheeseman, J. R.; Montgomery, J. A., Jr.; Vreven, T.; Kudin, K. N.; Burant, J. C.; Millam, J. M.; Iyengar, S. S.; Tomasi, J.; Barone, V.; Mennucci, B.; Cossi, M.; Scalmani, G.; Rega, N.; Petersson, G. A.; Nakatsuji, H.; Hada, M.; Ehara, M.; Toyota, K.; Fukuda, R.; Hasegawa, J.; Ishida, M.; Nakajima, T.; Honda, Y.; Kitao, O.; Nakai, H.; Klene, M.; Li, X.; Knox, J. E.; Hratchian, H. P.; Cross, J. B.; Bakken, V.; Adamo, C.; Jaramillo, J.; Gomperts, R.; Stratmann, R. E.; Yazyev, O.; Austin, A. J.; Cammi, R.; Pomelli, C.; Ochterski, J. W.; Ayala, P. Y.; Morokuma, K.; Voth, G. A.; Salvador, P.; Dannenberg, J. J.; Zakrzewski, V. G.; Dapprich, S.; Daniels, A. D.; Strain, M. C.; Farkas, O.; Malick, D. K.; Rabuck, A. D.; Raghavachari, K.; Foresman, J. B.; Ortiz, J. V.; Cui, Q.; Baboul, A. G.; Clifford, S.; Cioslowski, J.; Stefanov, B. B.; Liu, G.; Liashenko, A.; Piskorz, P.; Komaromi, I.; Martin, R. L.; Fox, D. J.; Keith, T.; Al-Laham, M. A.; Peng, C. Y.; Nanayakkara, A.; Challacombe, M.; Gill, P. M. W.; Johnson, B.; Chen, W.; Wong, M. W.; Gonzalez, C.; Pople, J. A. Gaussian 03, revision C.02; Gaussian, Inc.: Wallingford, CT, 2004. 


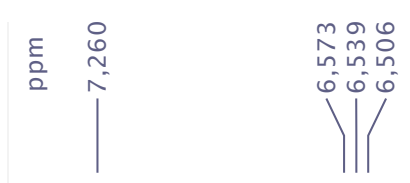
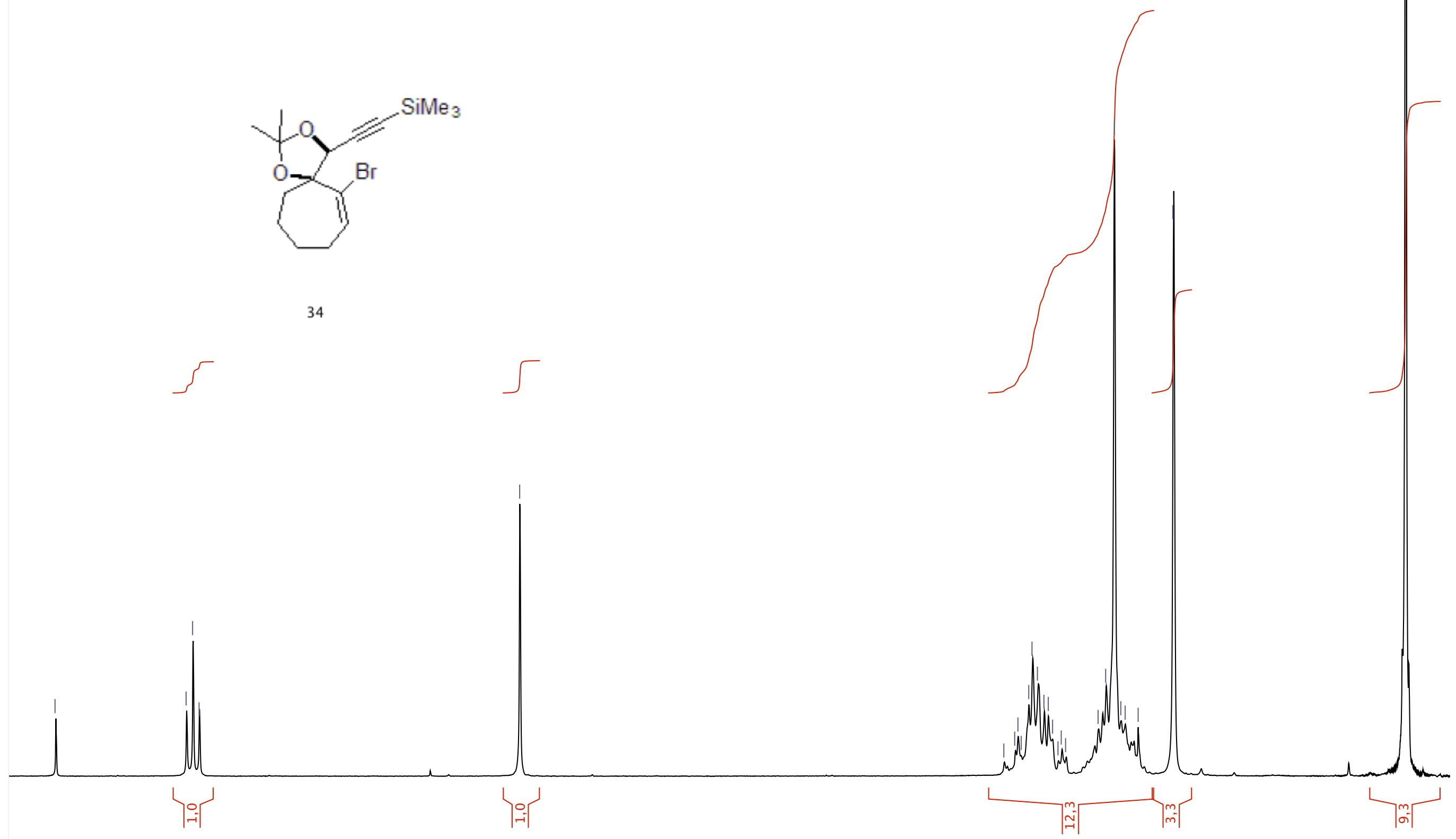


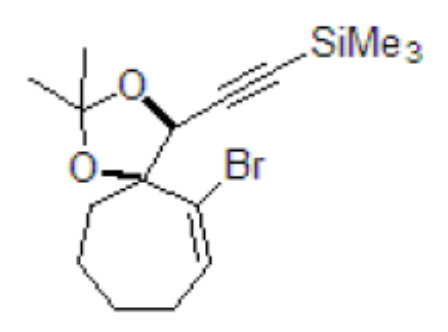

34

(n)

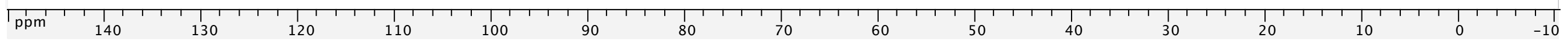




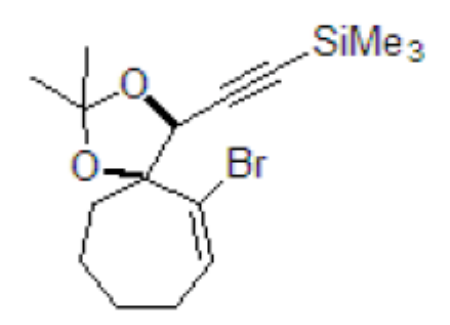

34

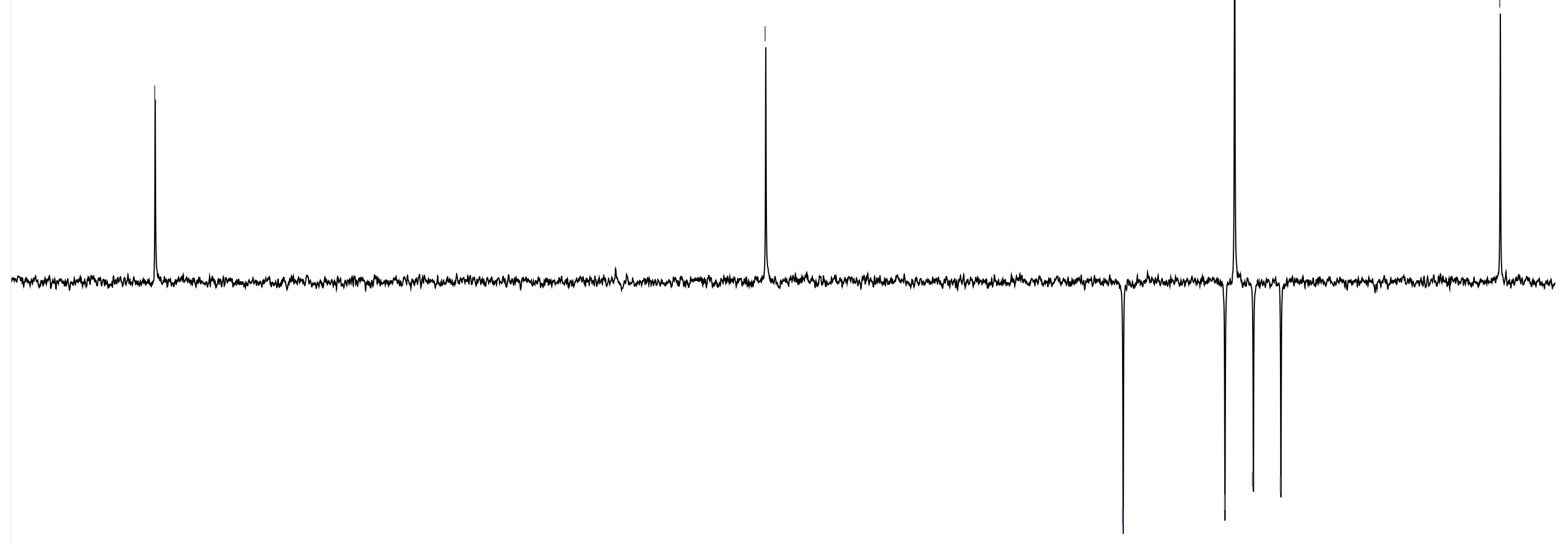


|气

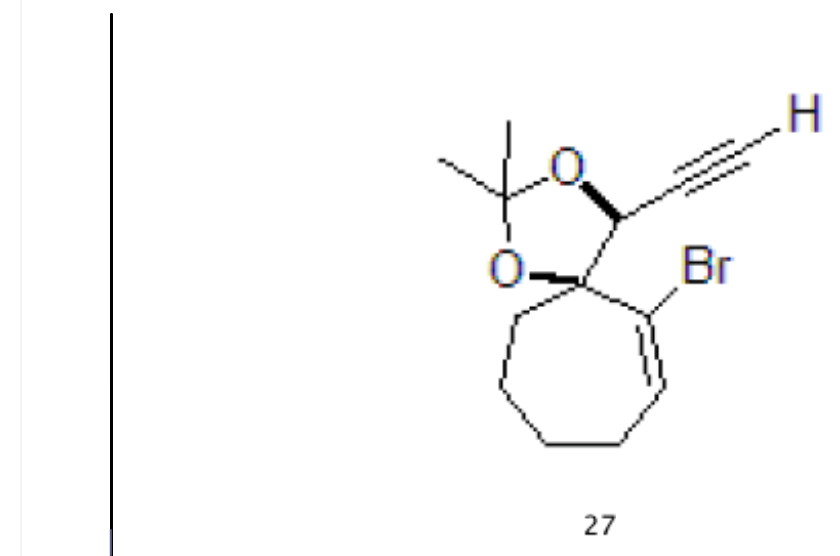

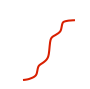

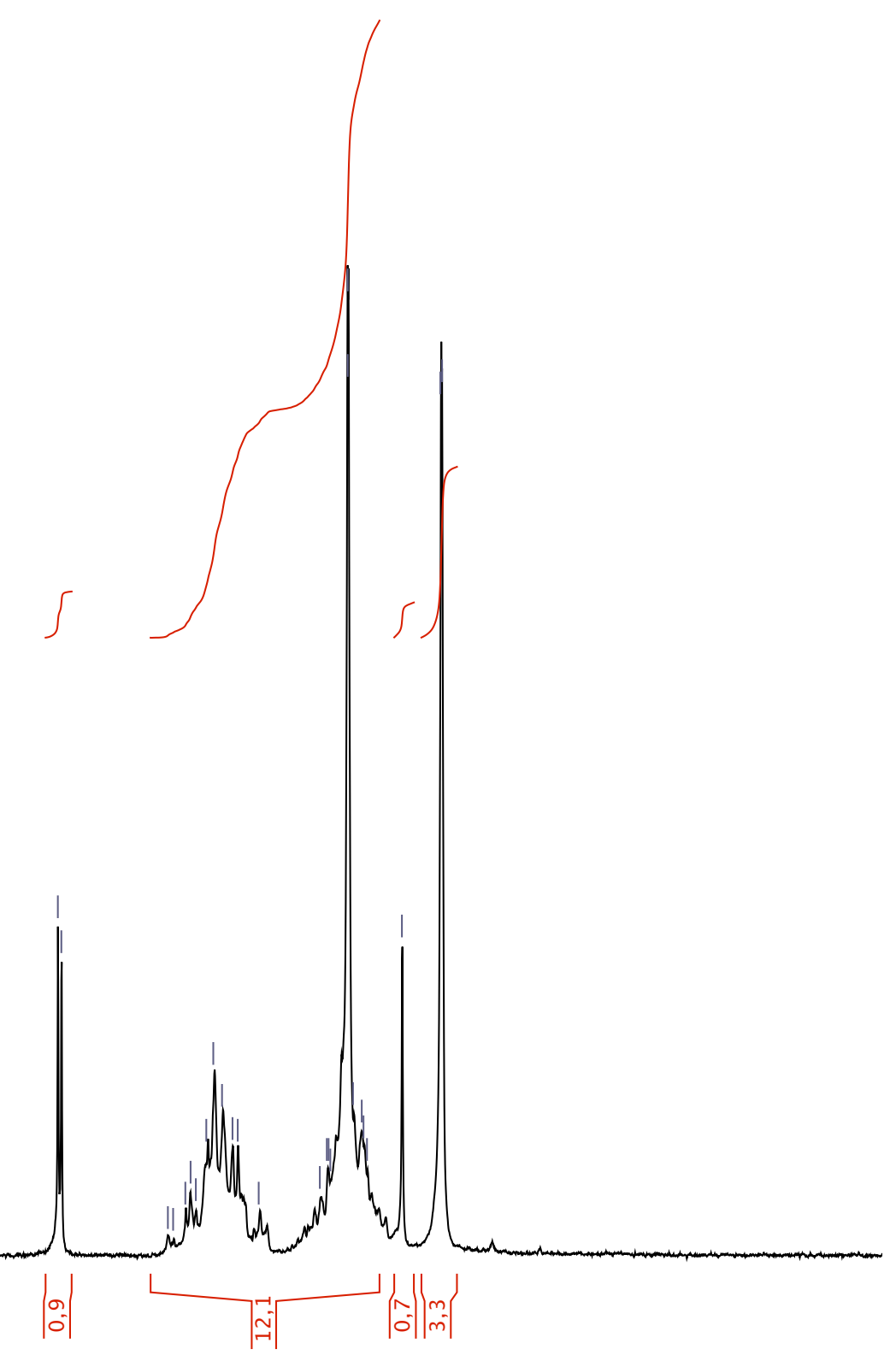




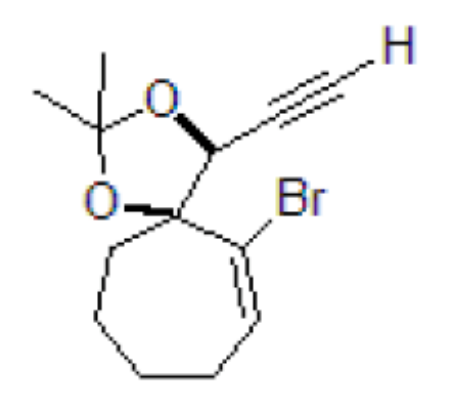

27

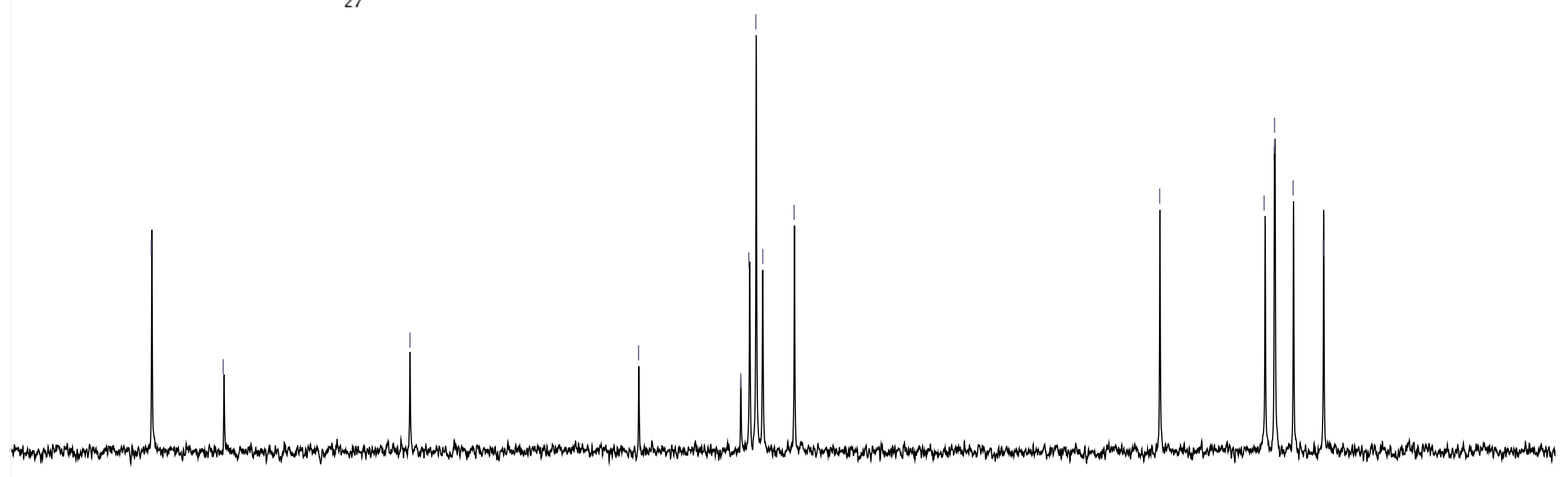




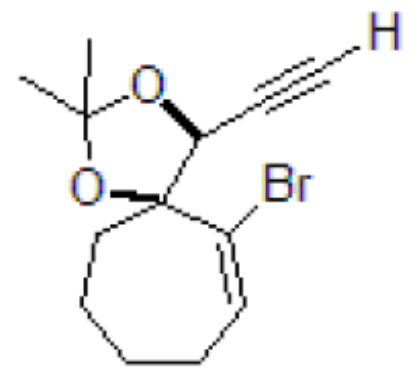

27

(1) 


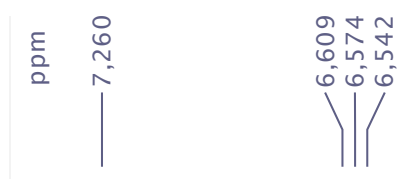
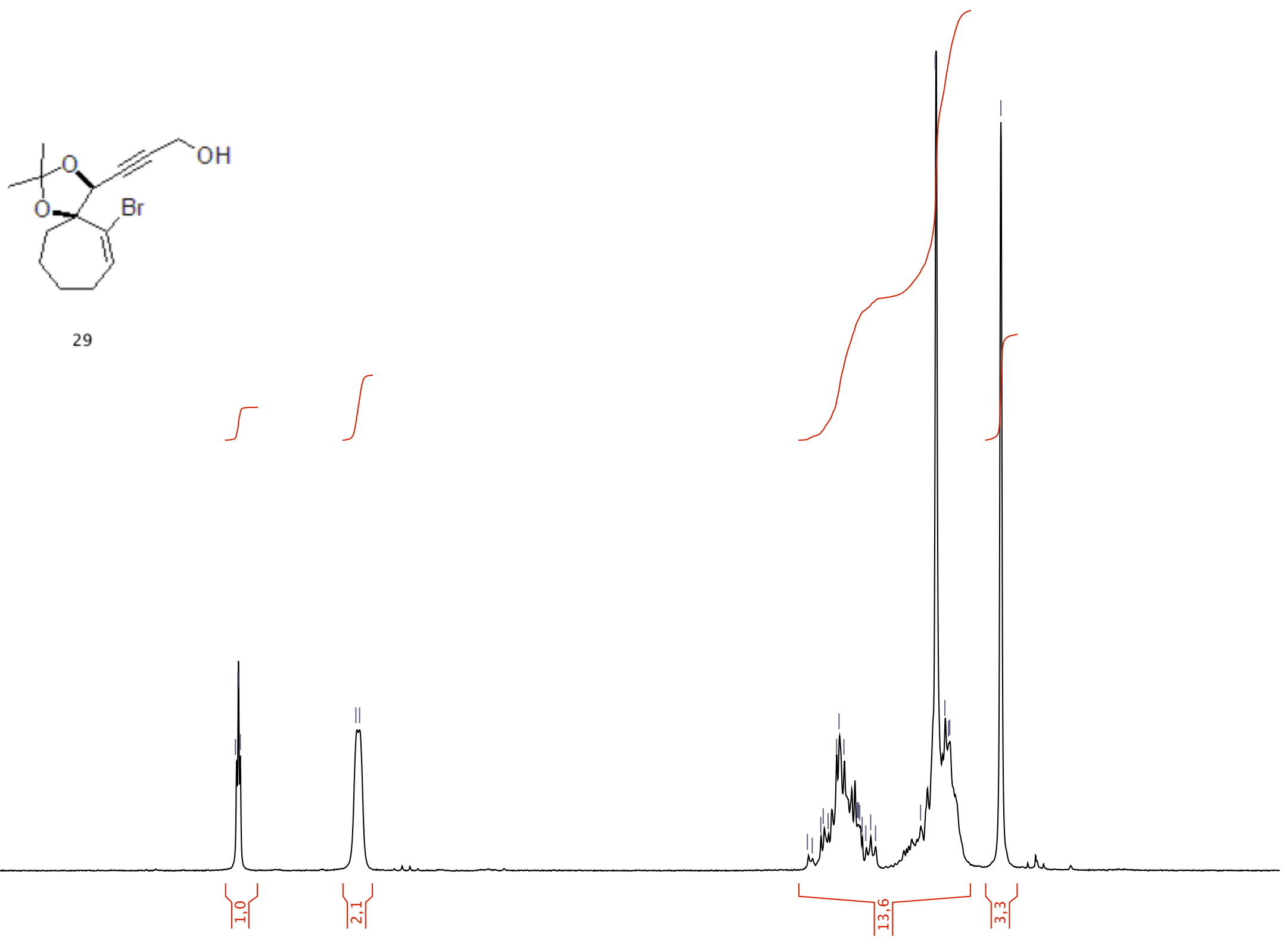
1
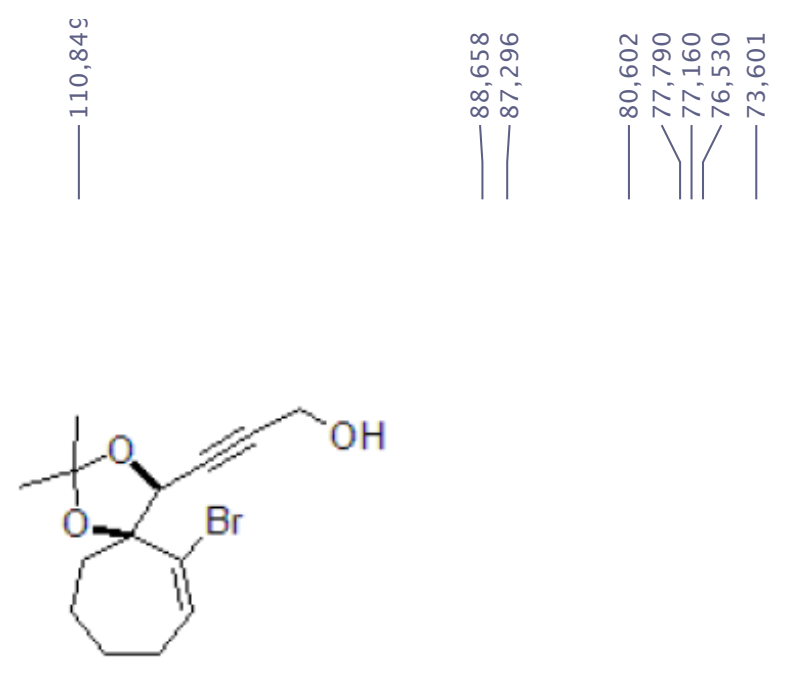

29

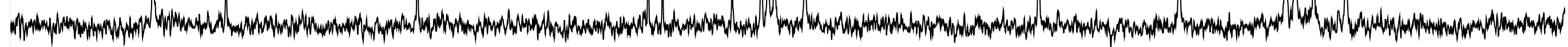




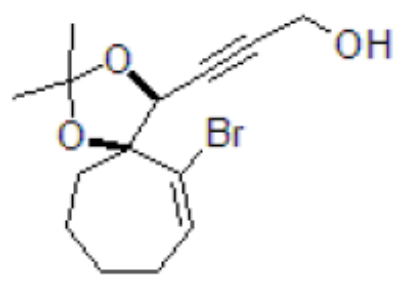

29

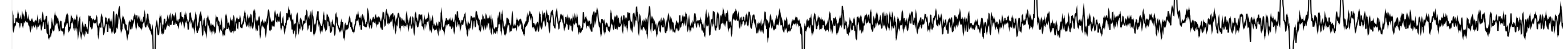




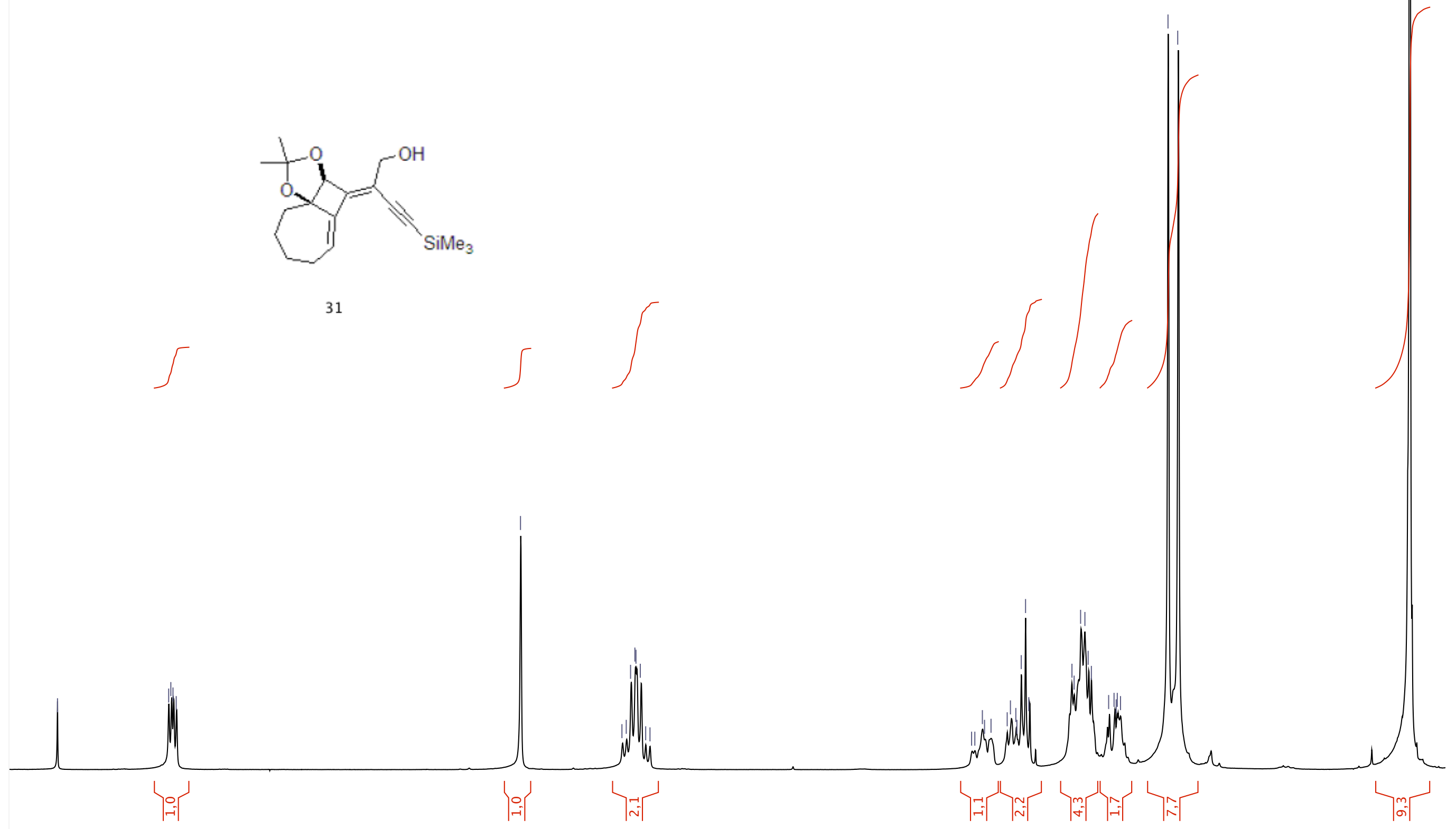




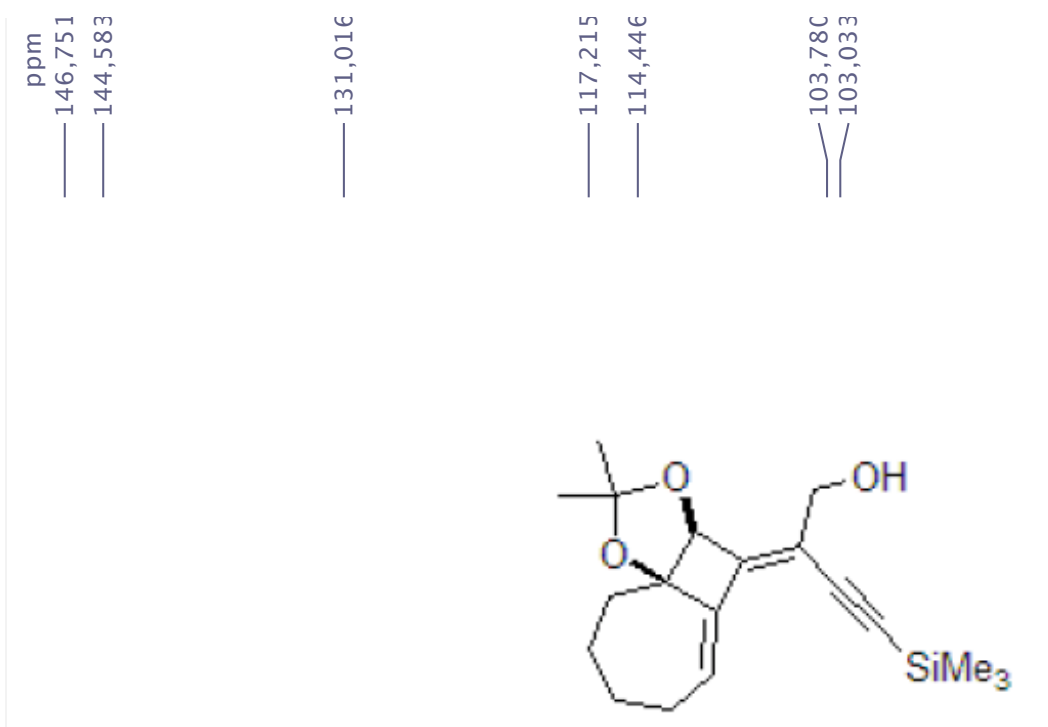

31
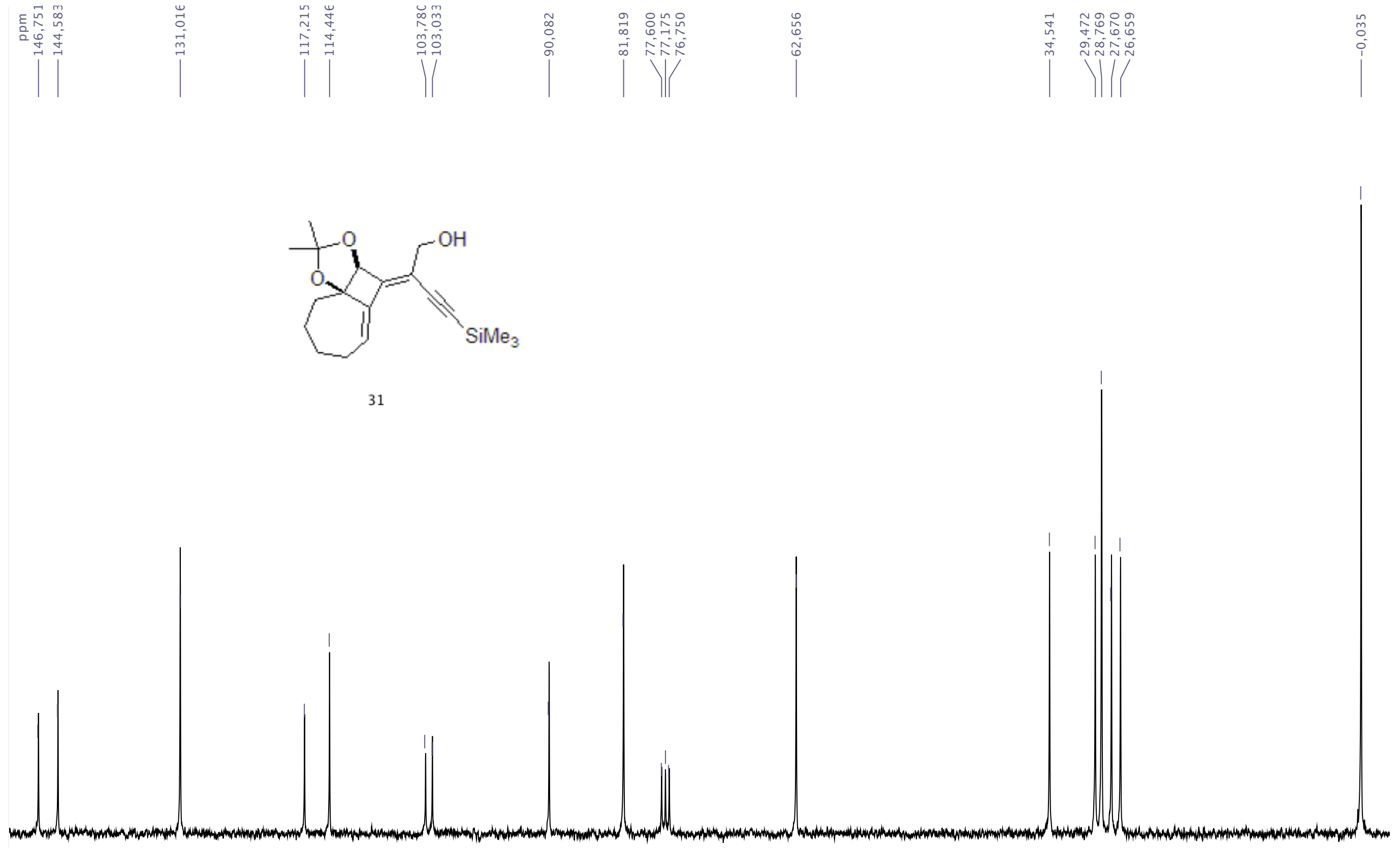


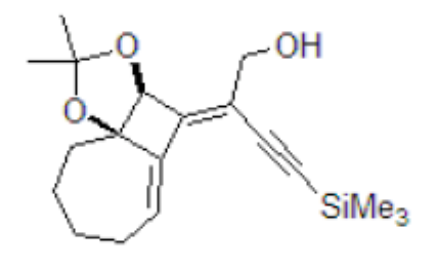

31

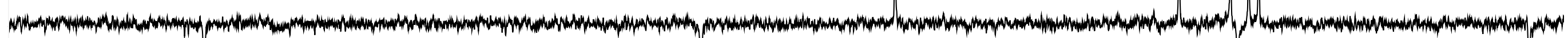
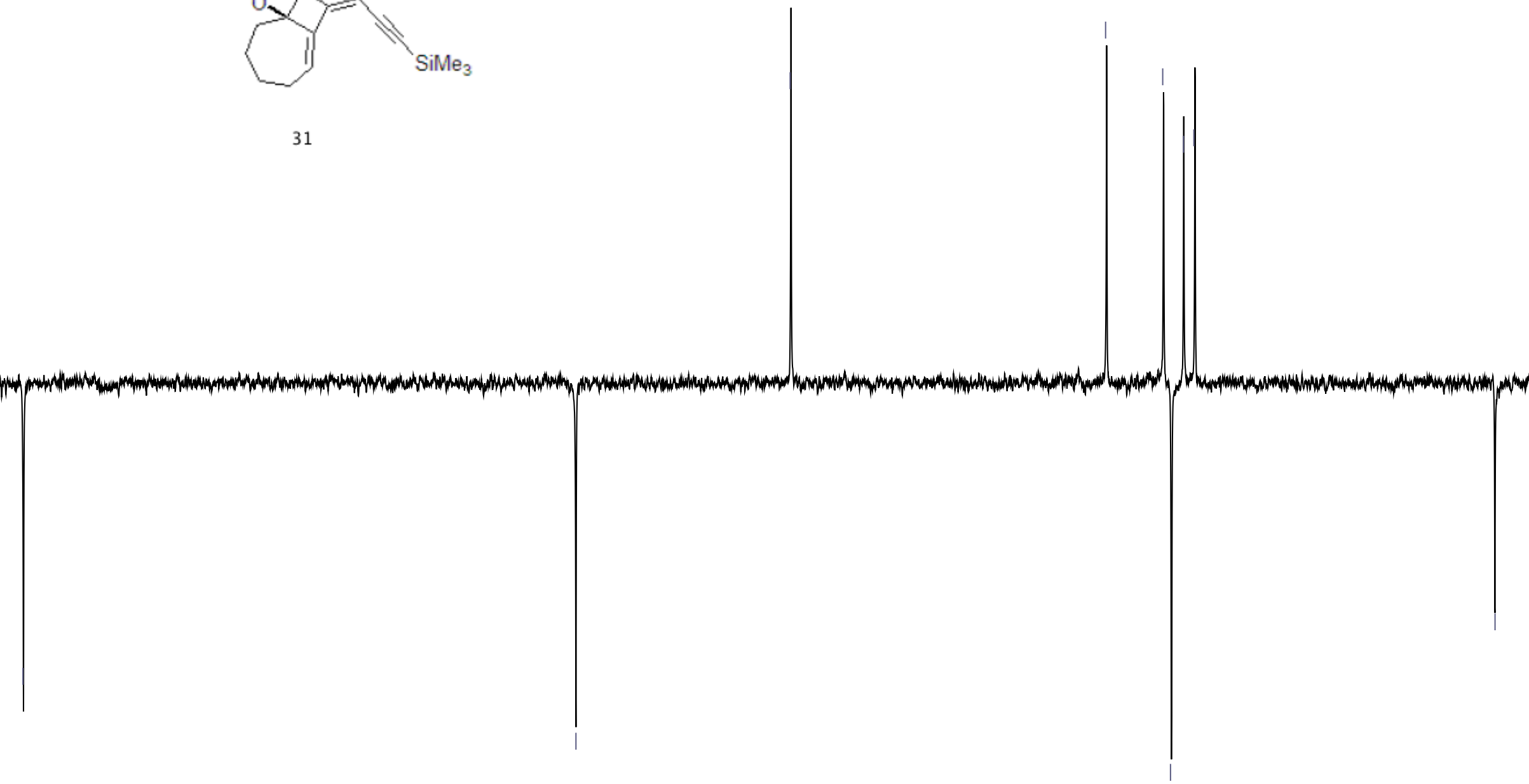


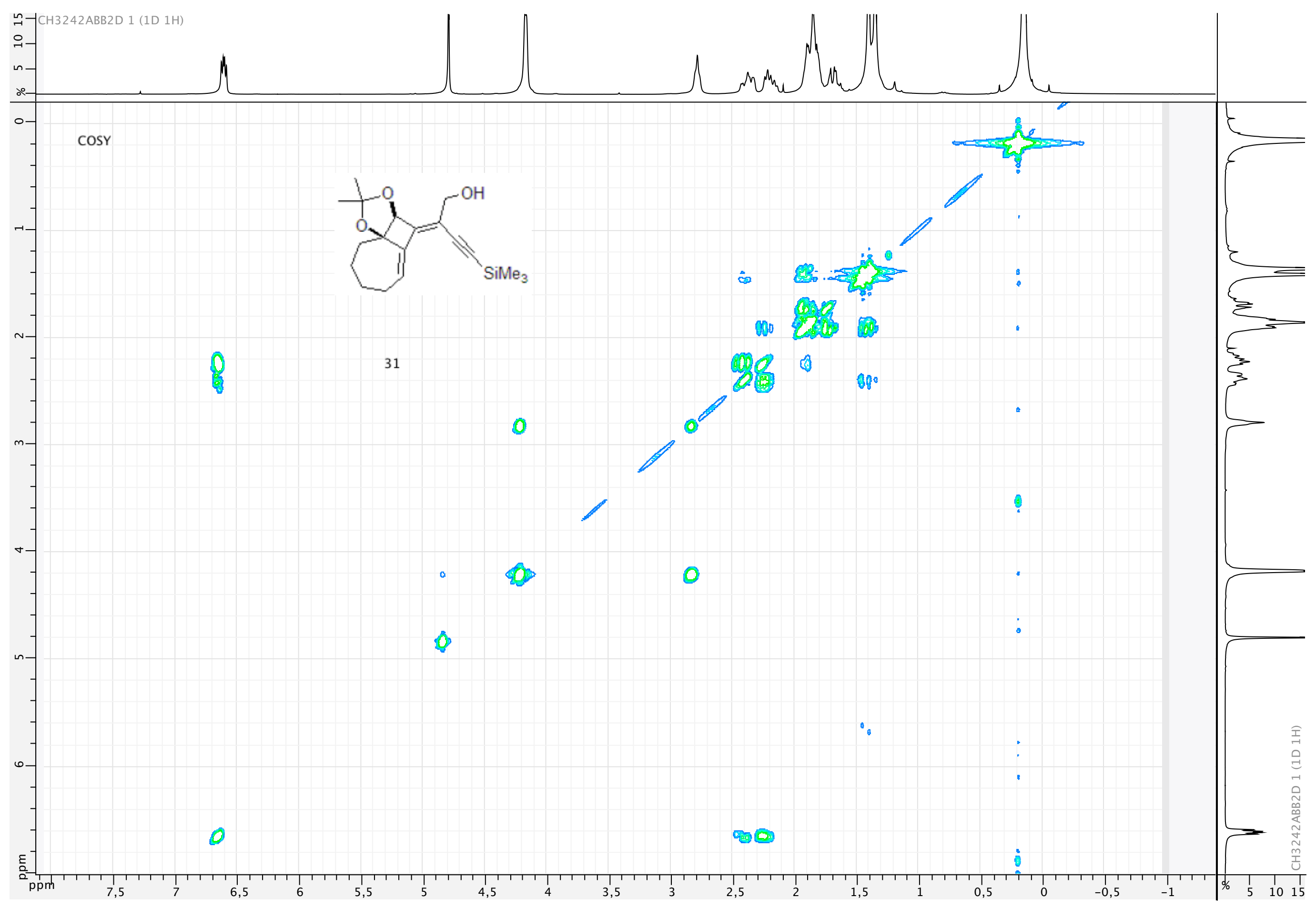




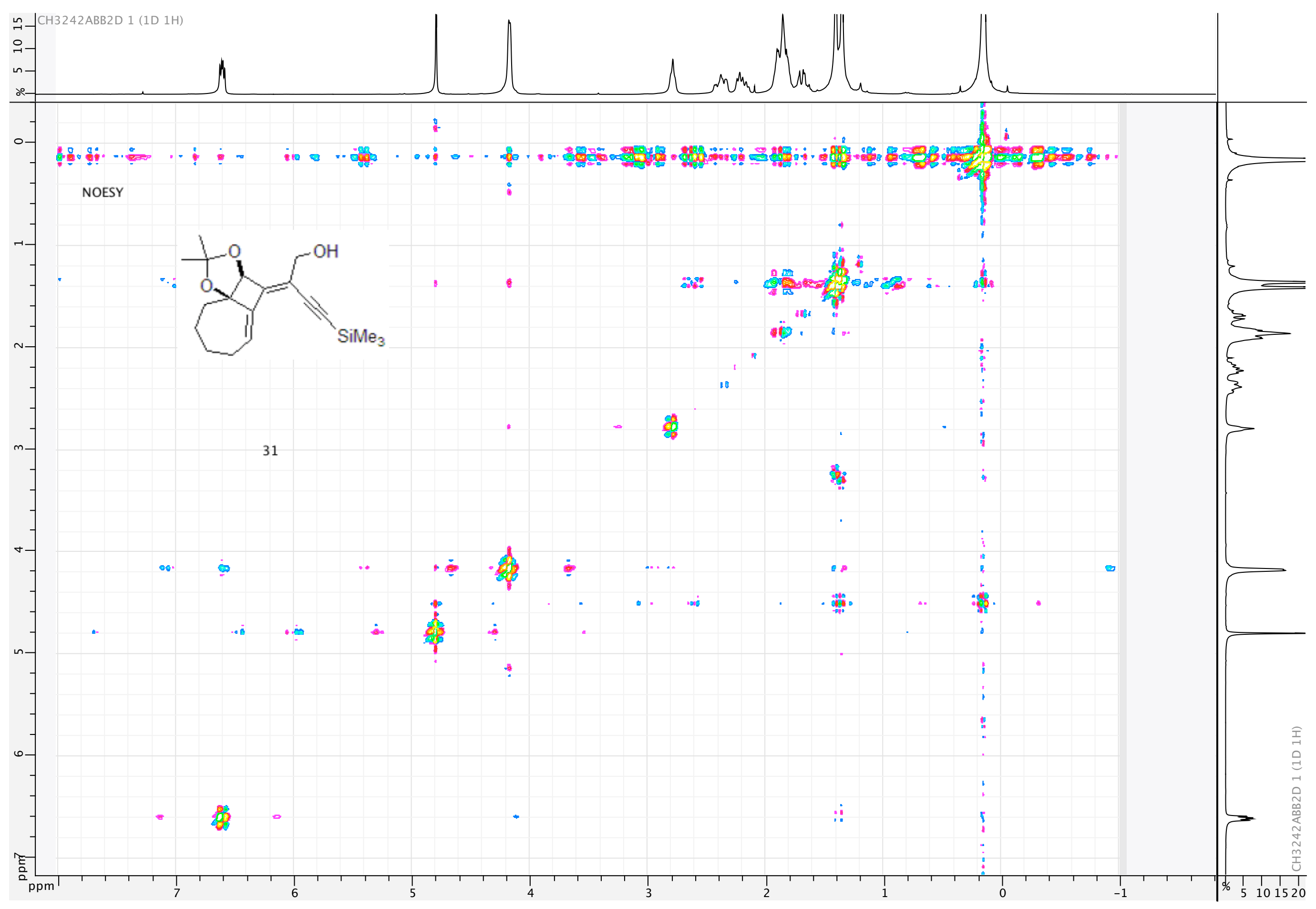




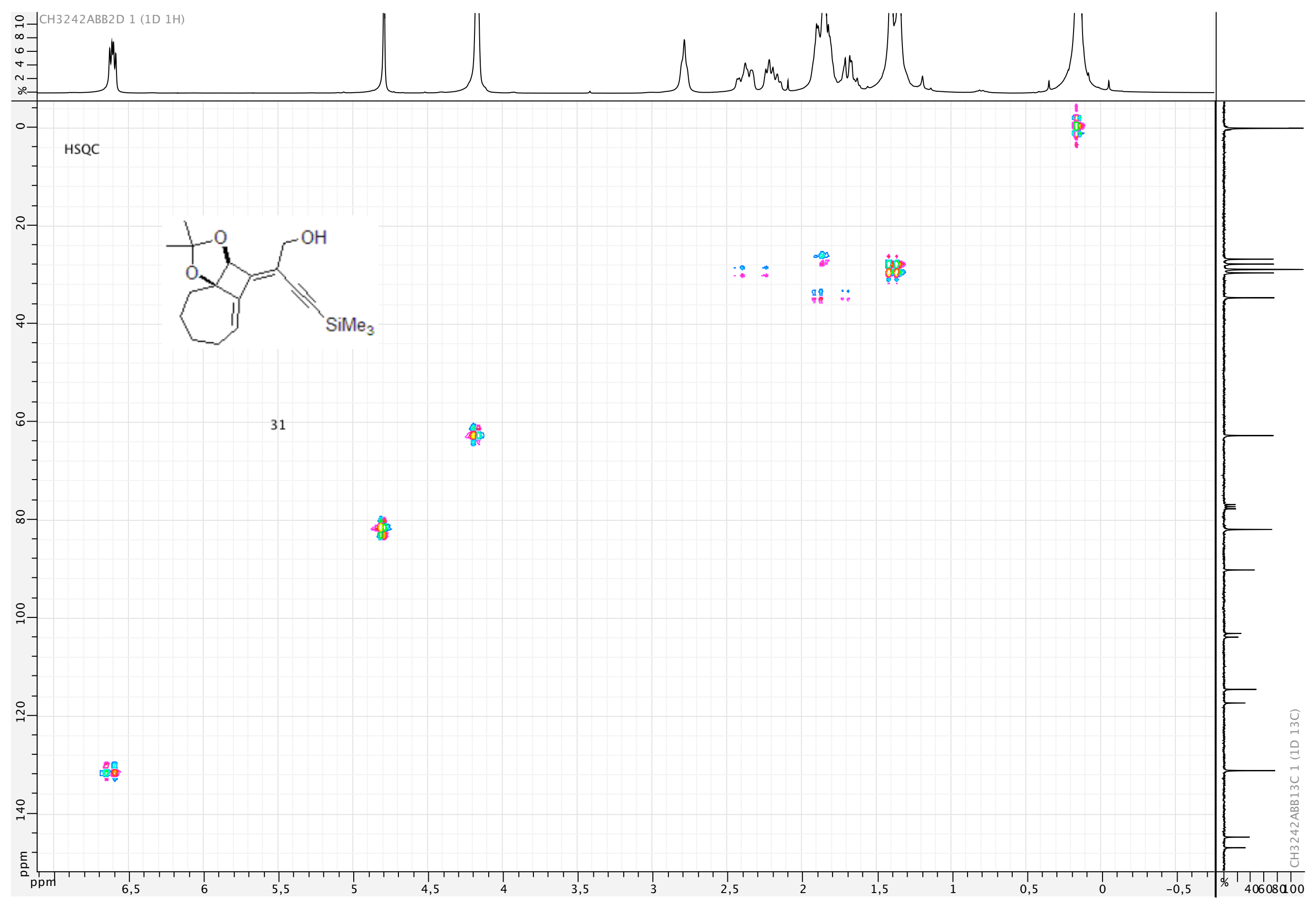




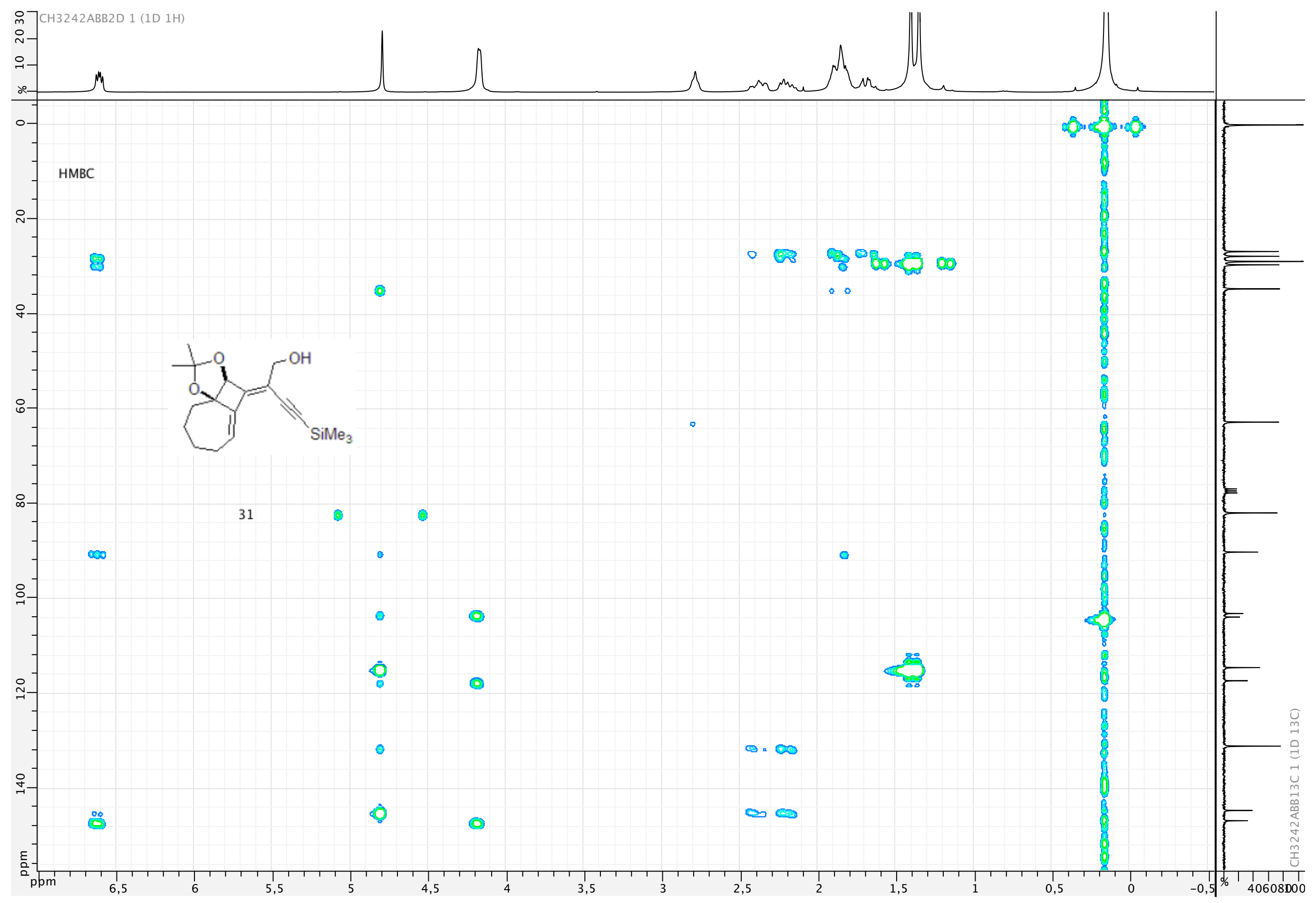


|

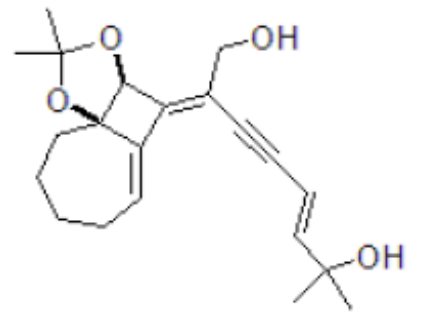

$5 a$
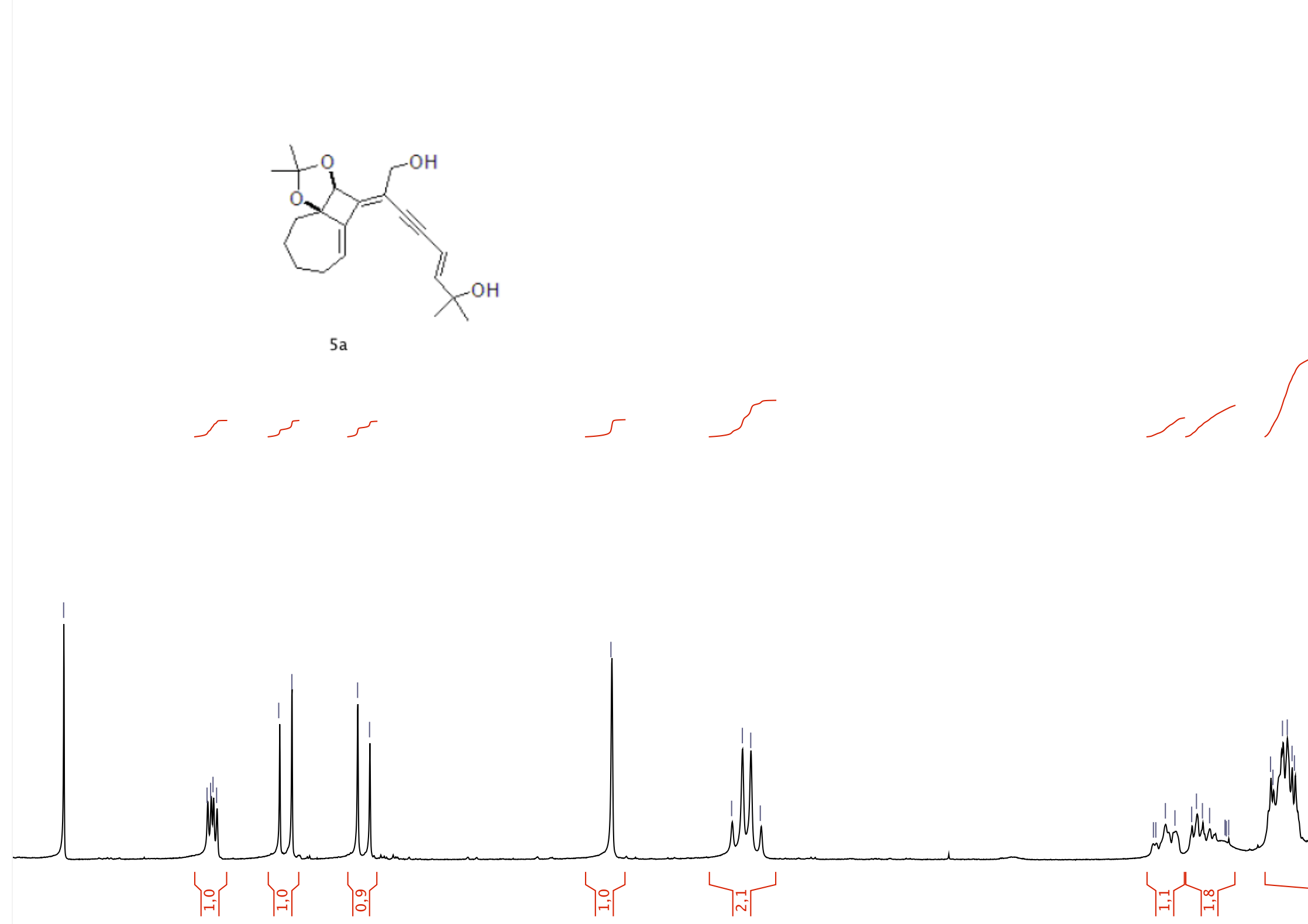


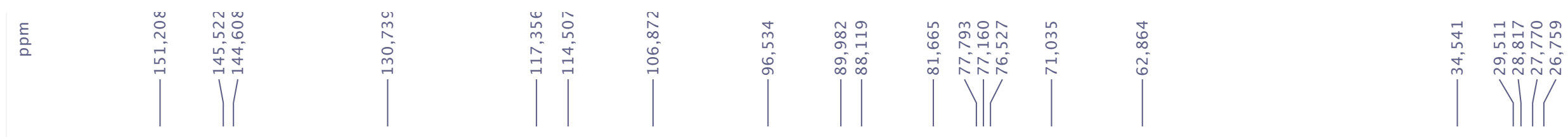

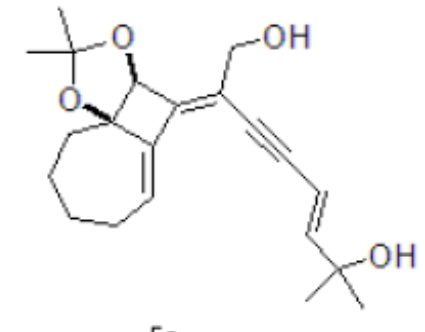

$5 a$

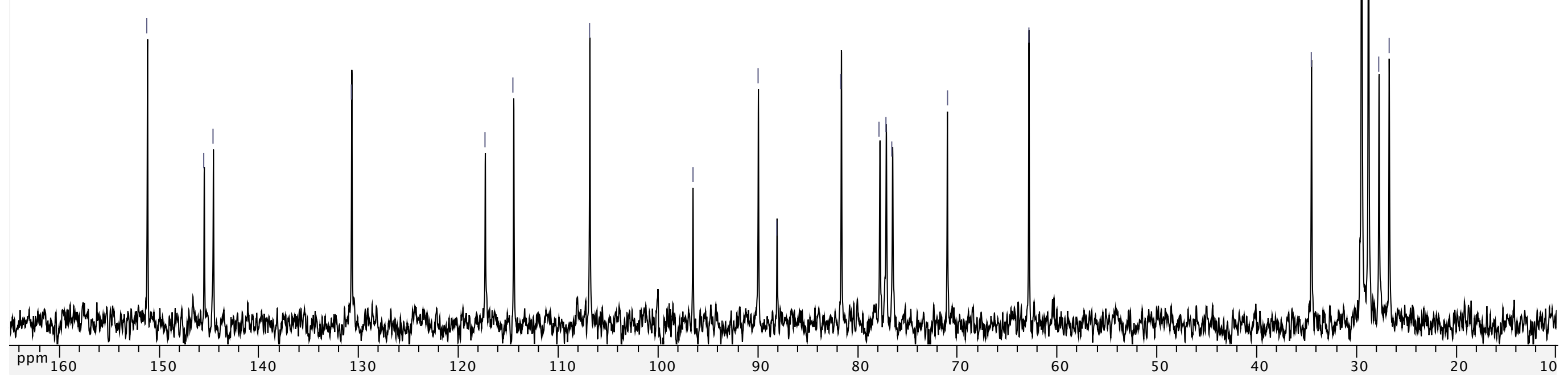




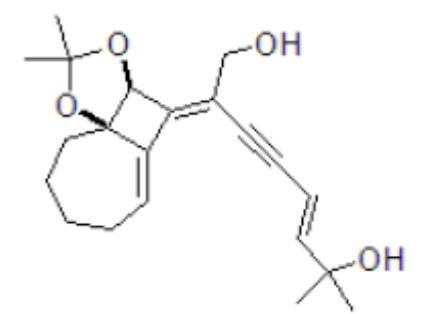

$5 a$

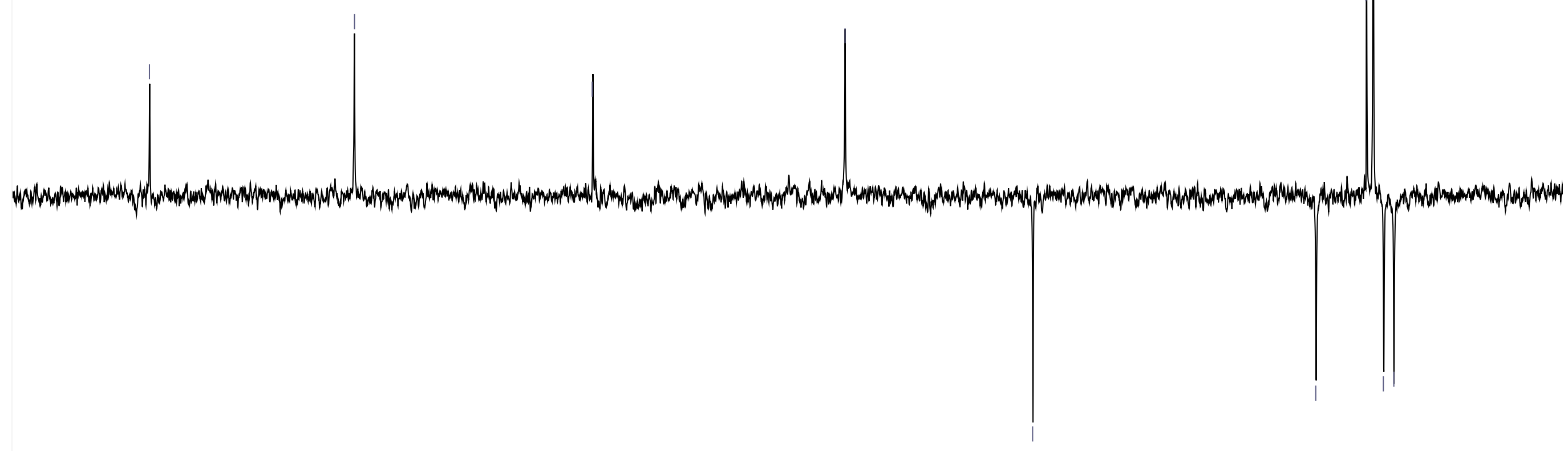




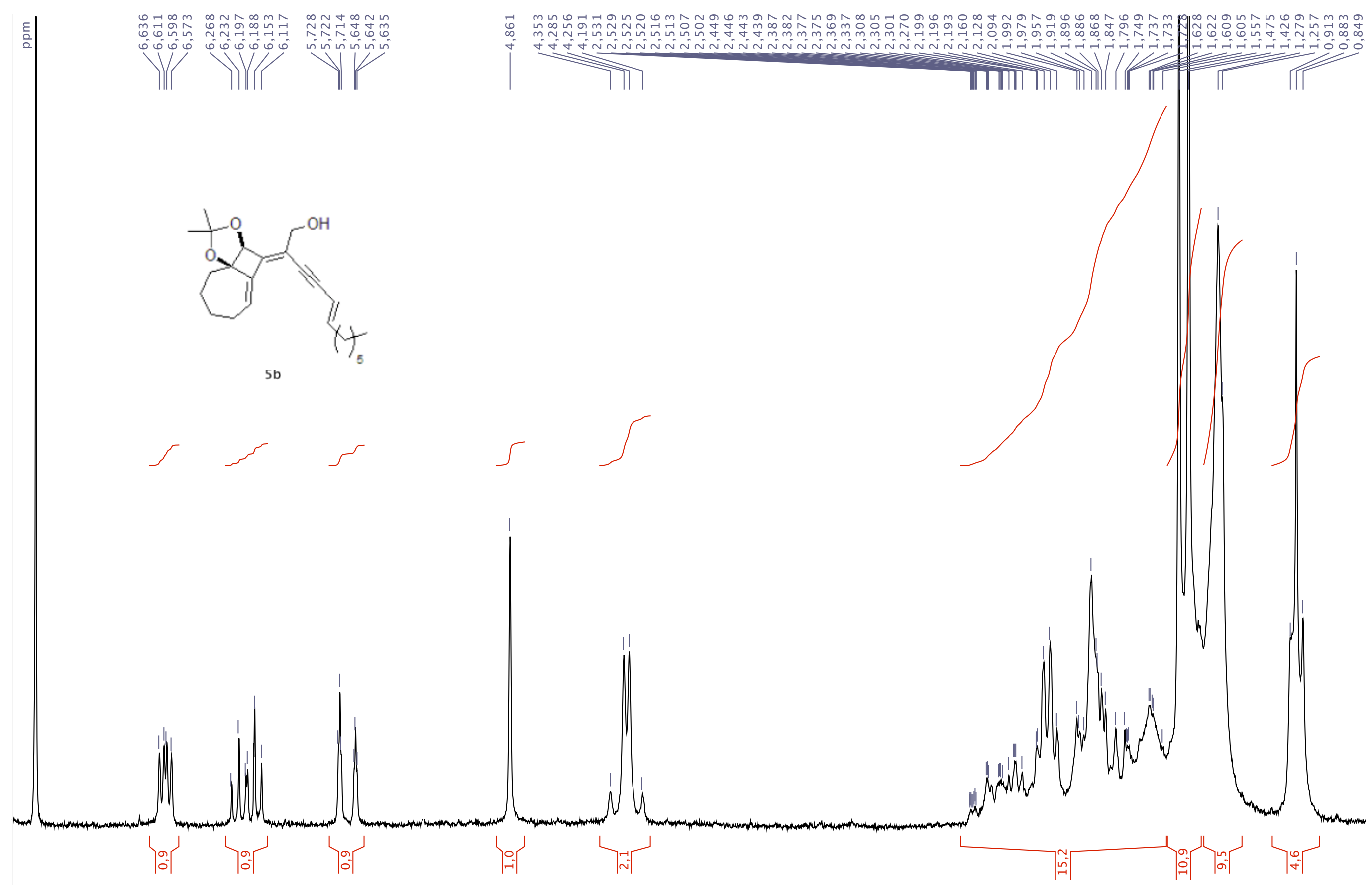




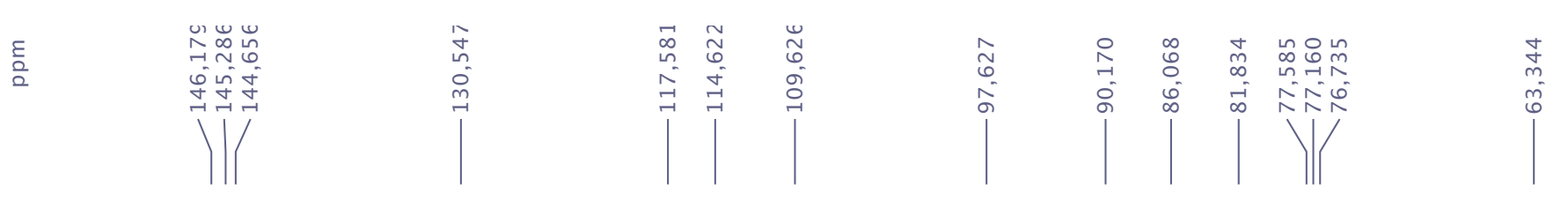

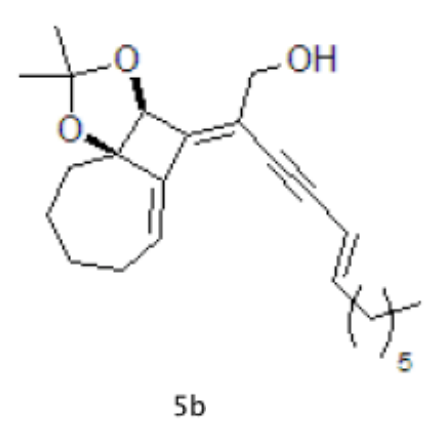

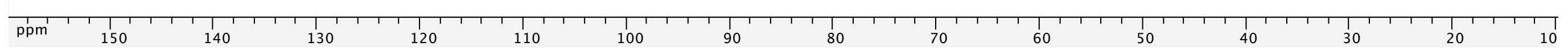


高䲞

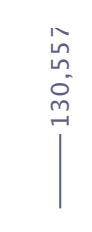

๙ึ.

i

11)

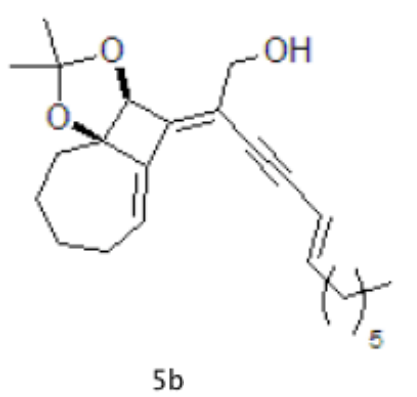




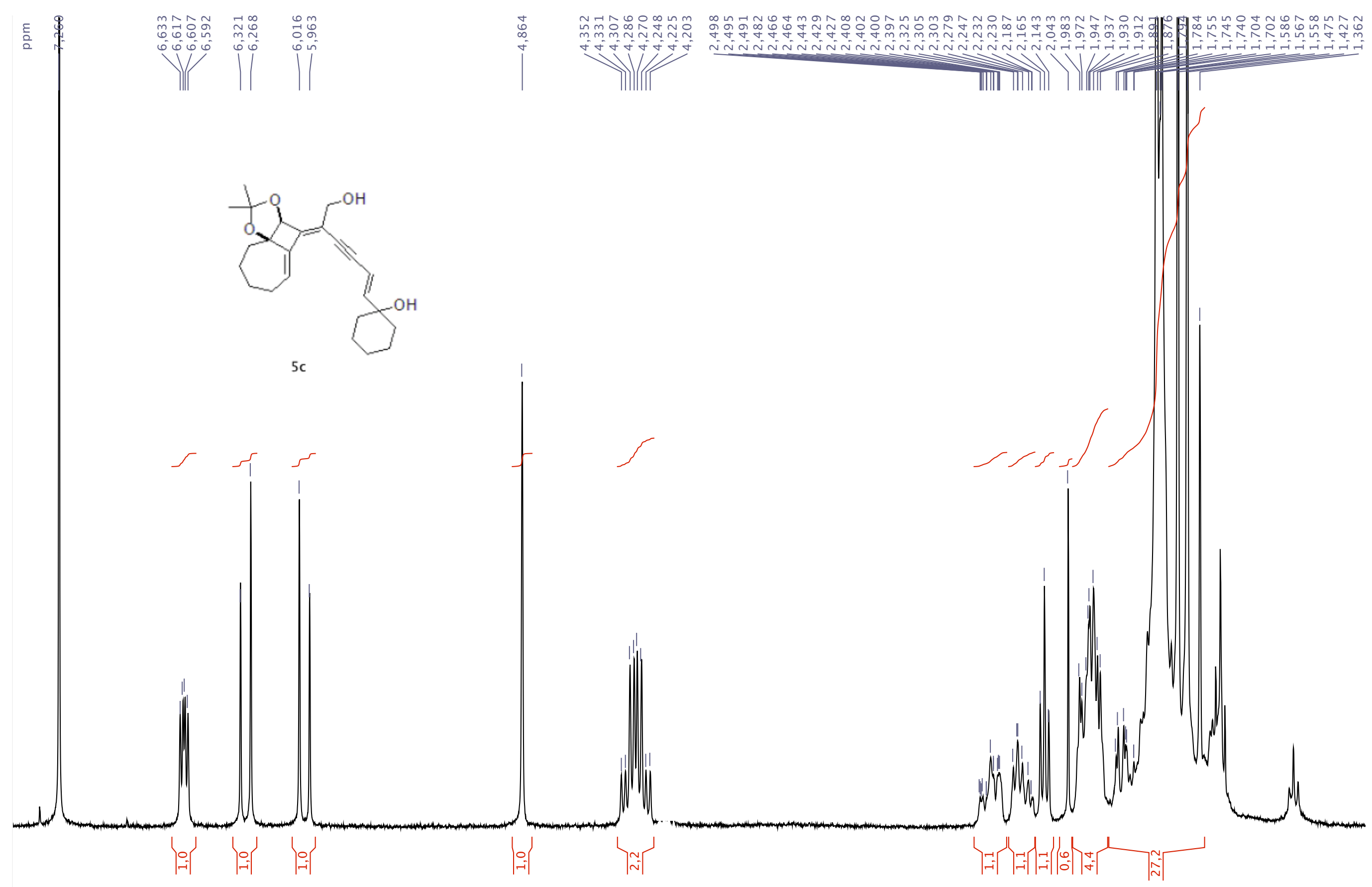



1
率旁

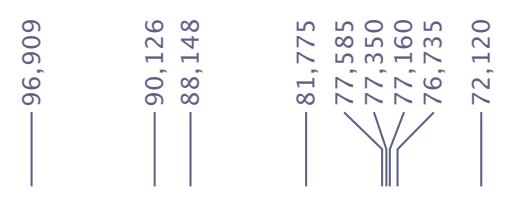

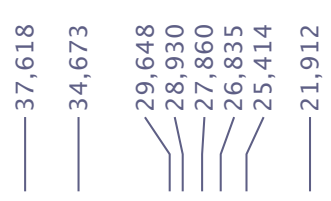

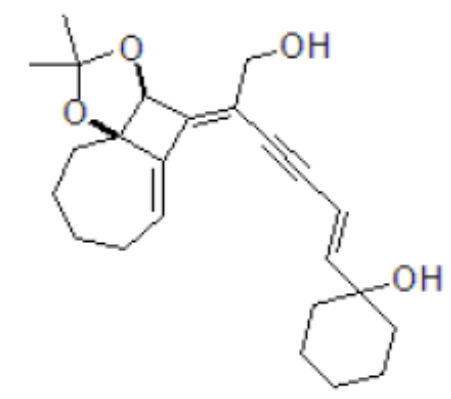

$5 c$

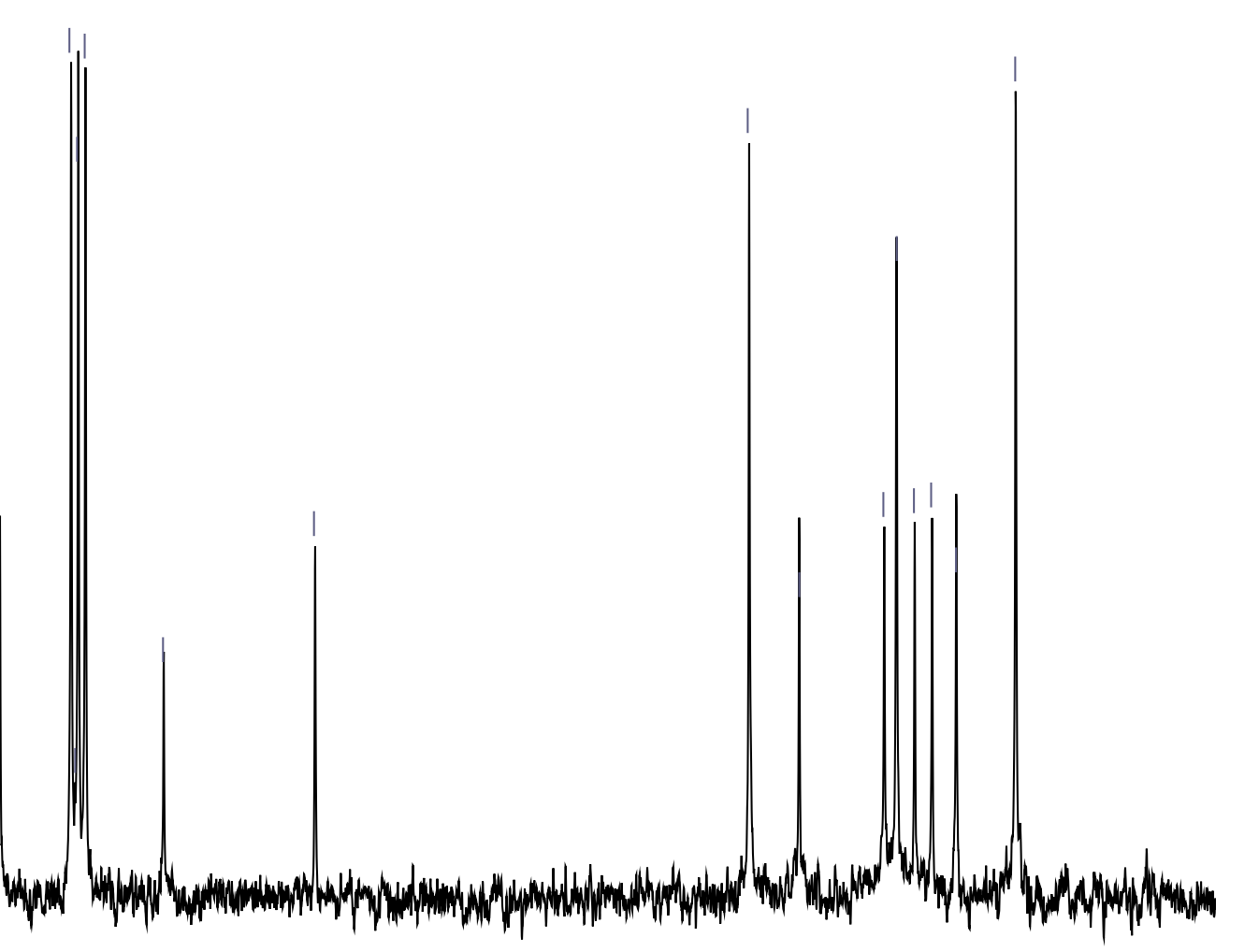


ह

$\left.\right|_{\substack{f \\ 0 \\ 0}} ^{\substack{0 \\ 0}}$

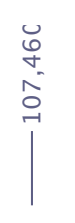

$\underset{\substack { n \\ \begin{subarray}{c}{0 \\ \infty{ n \\ \begin{subarray} { c } { 0 \\ \infty } }\end{subarray}}{\substack{0 \\ 0}}$

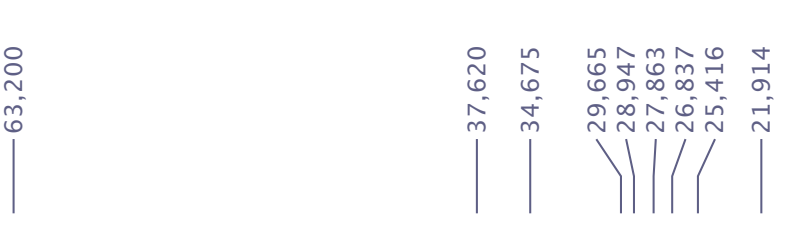

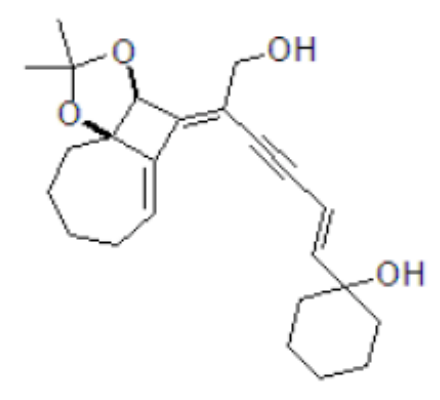

$5 c$

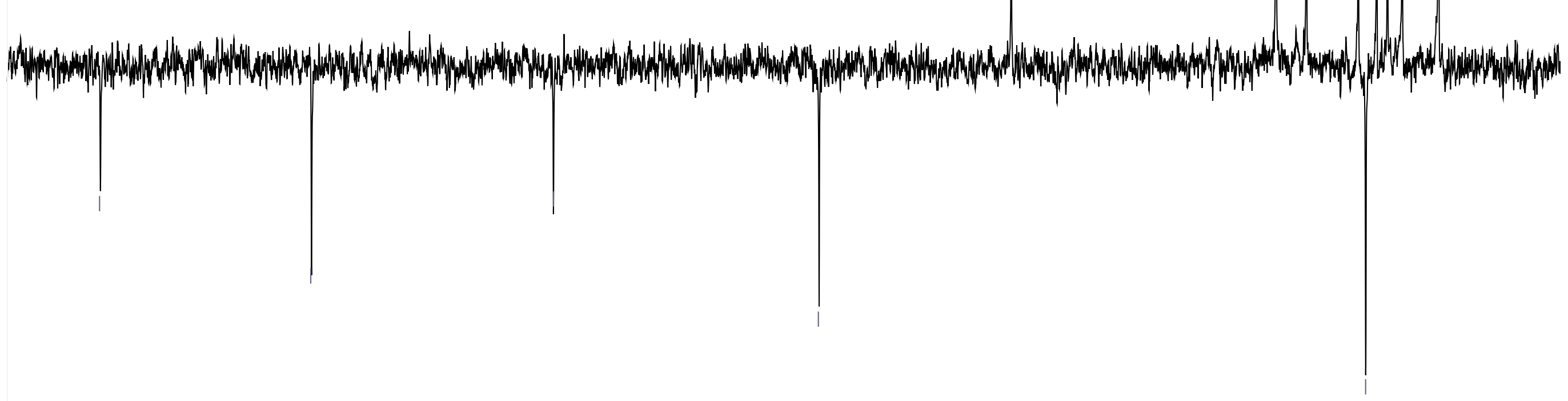

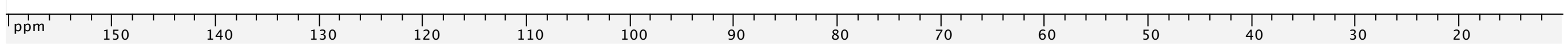


气

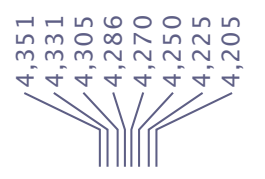

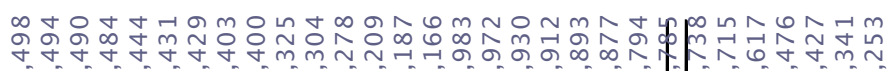

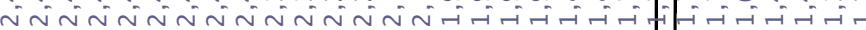

(n) $\|) / / C$

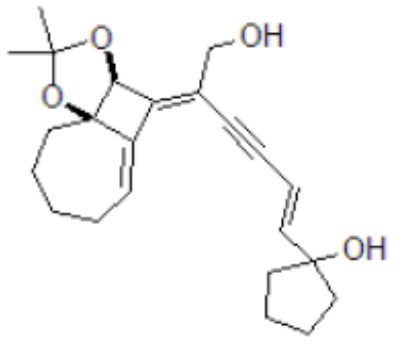

$5 d$

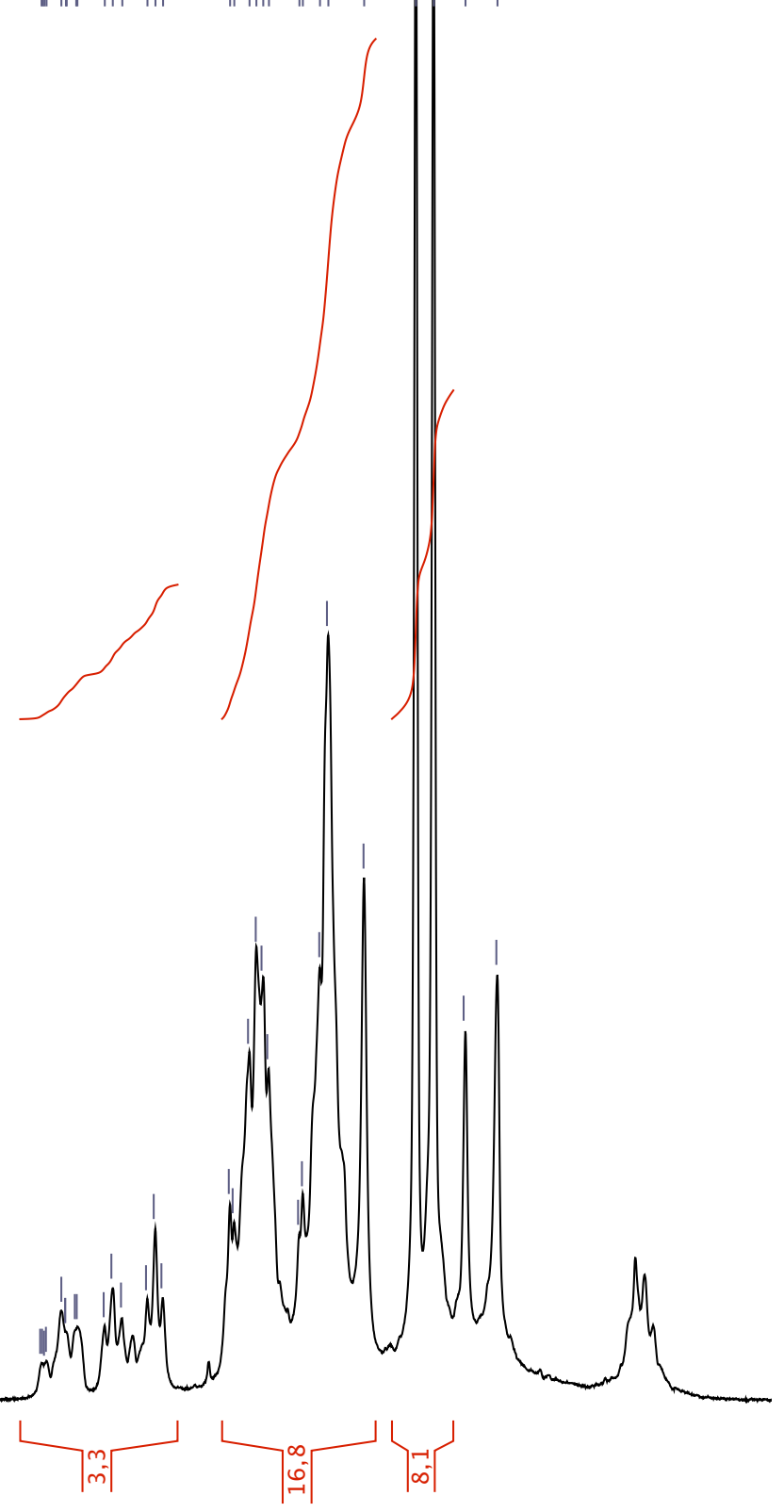




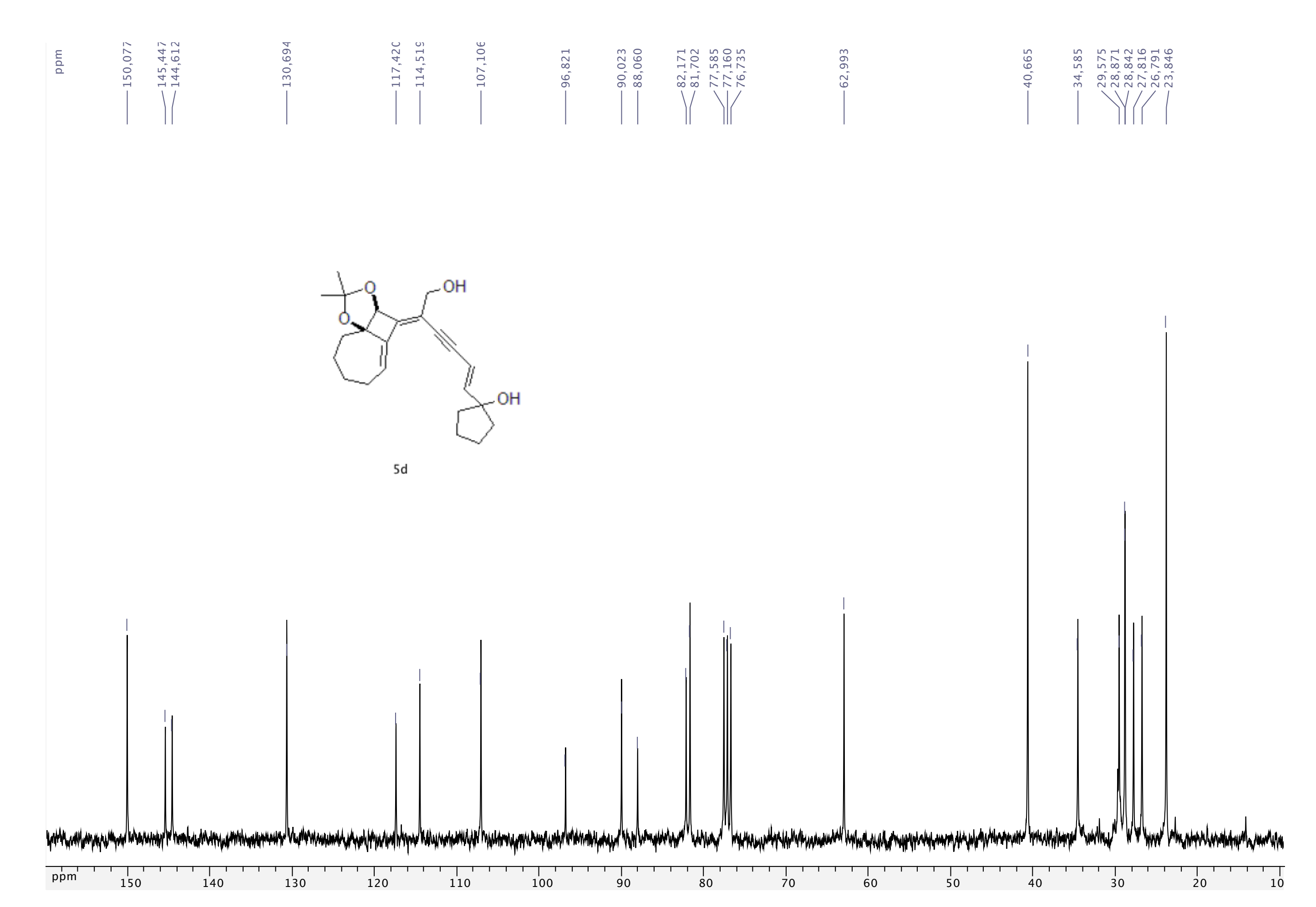


ह

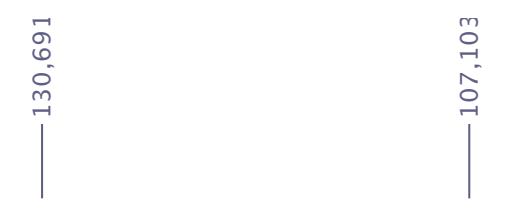

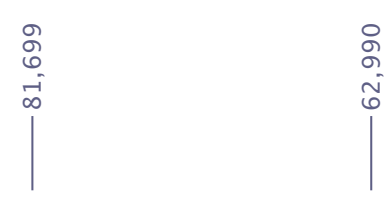

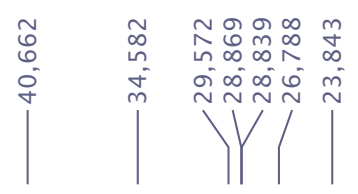

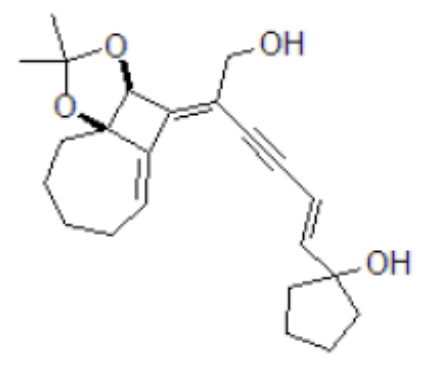

$5 d$

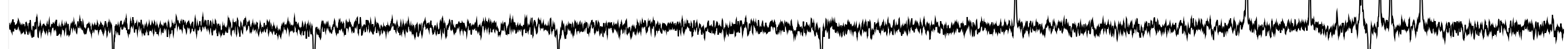
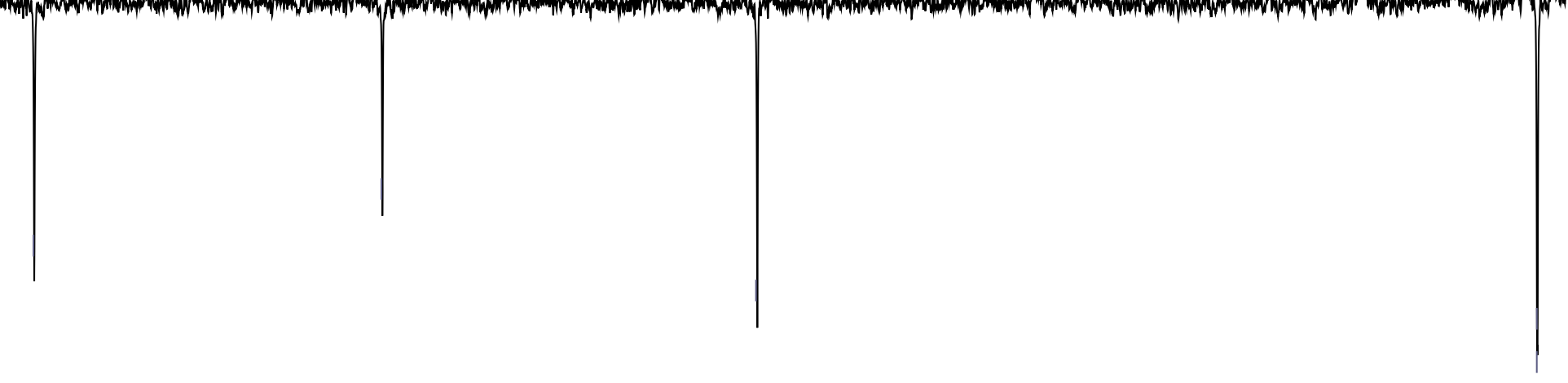


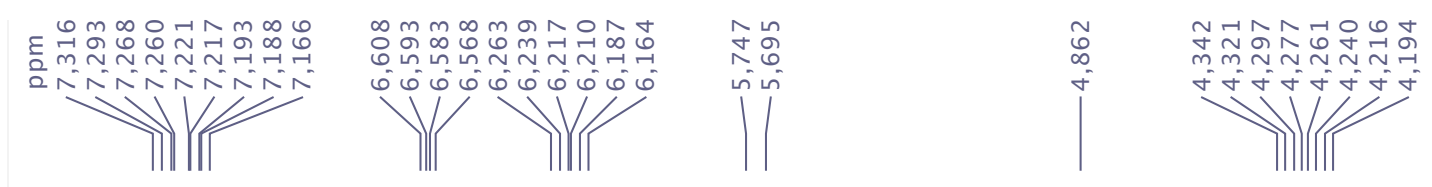

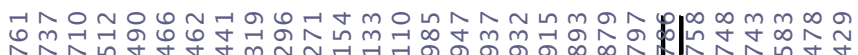
पा\| \|\|\|\|$\|$
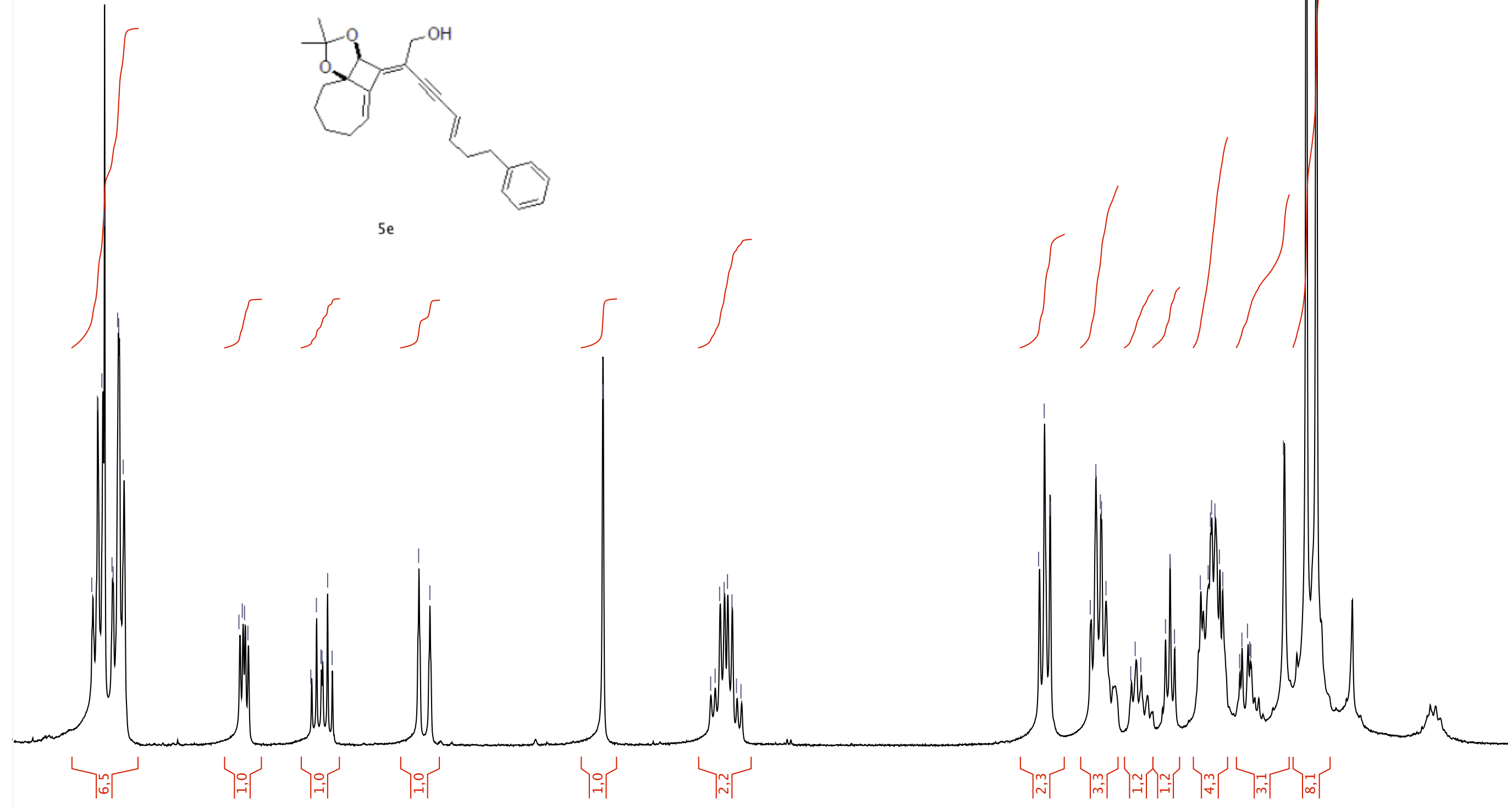


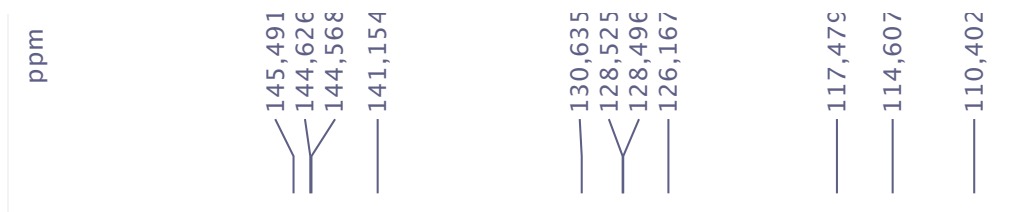

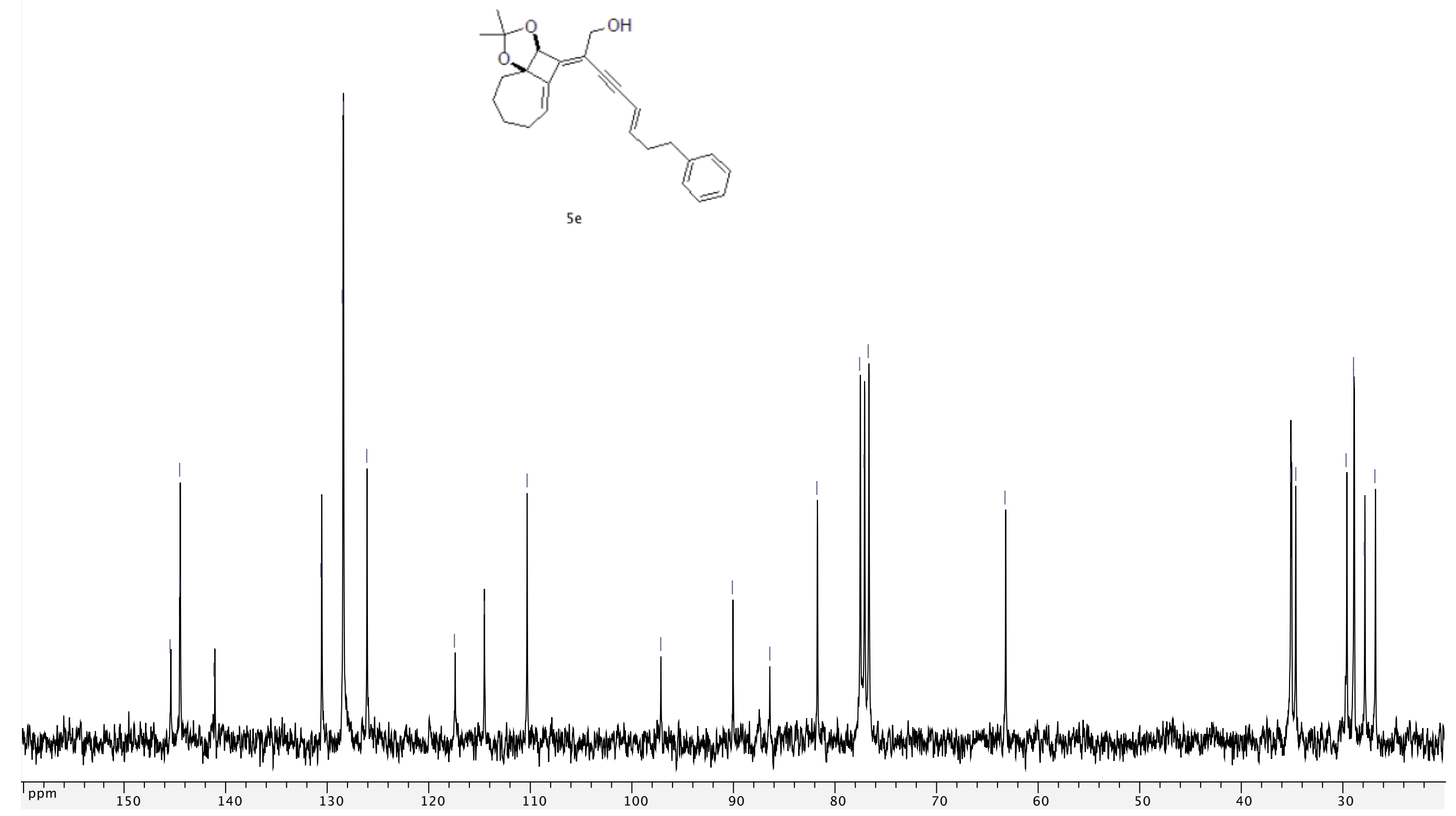



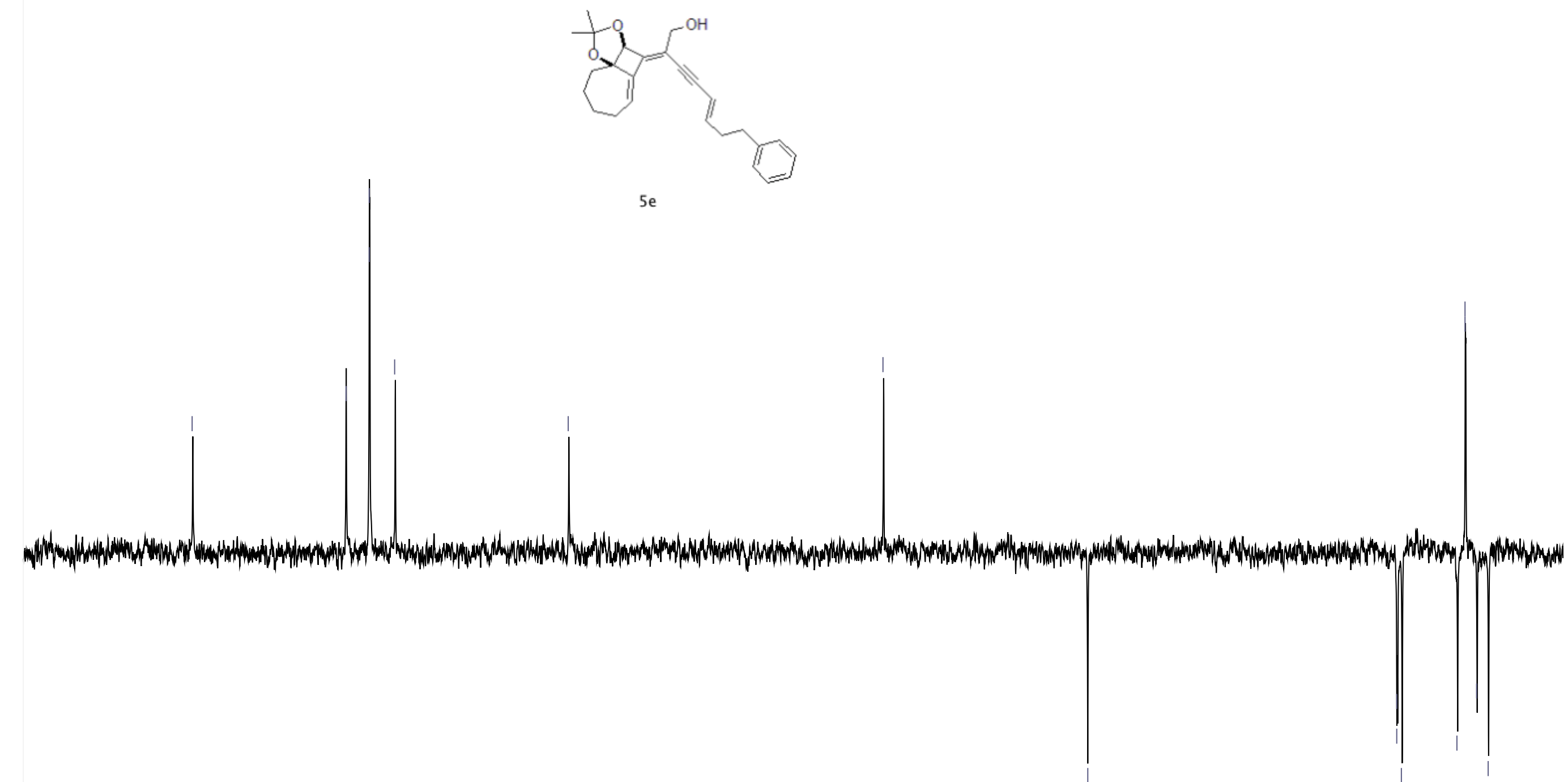


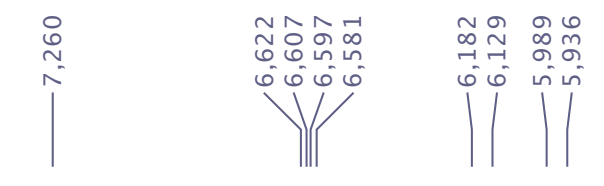

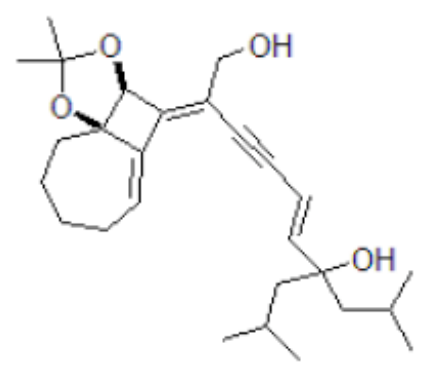

$5 f$

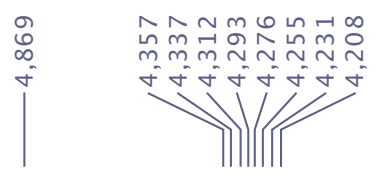

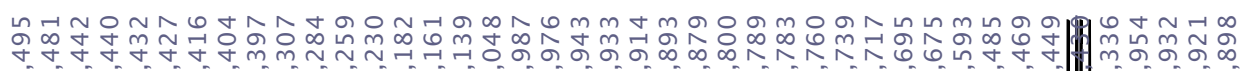

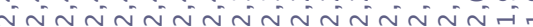
(1)
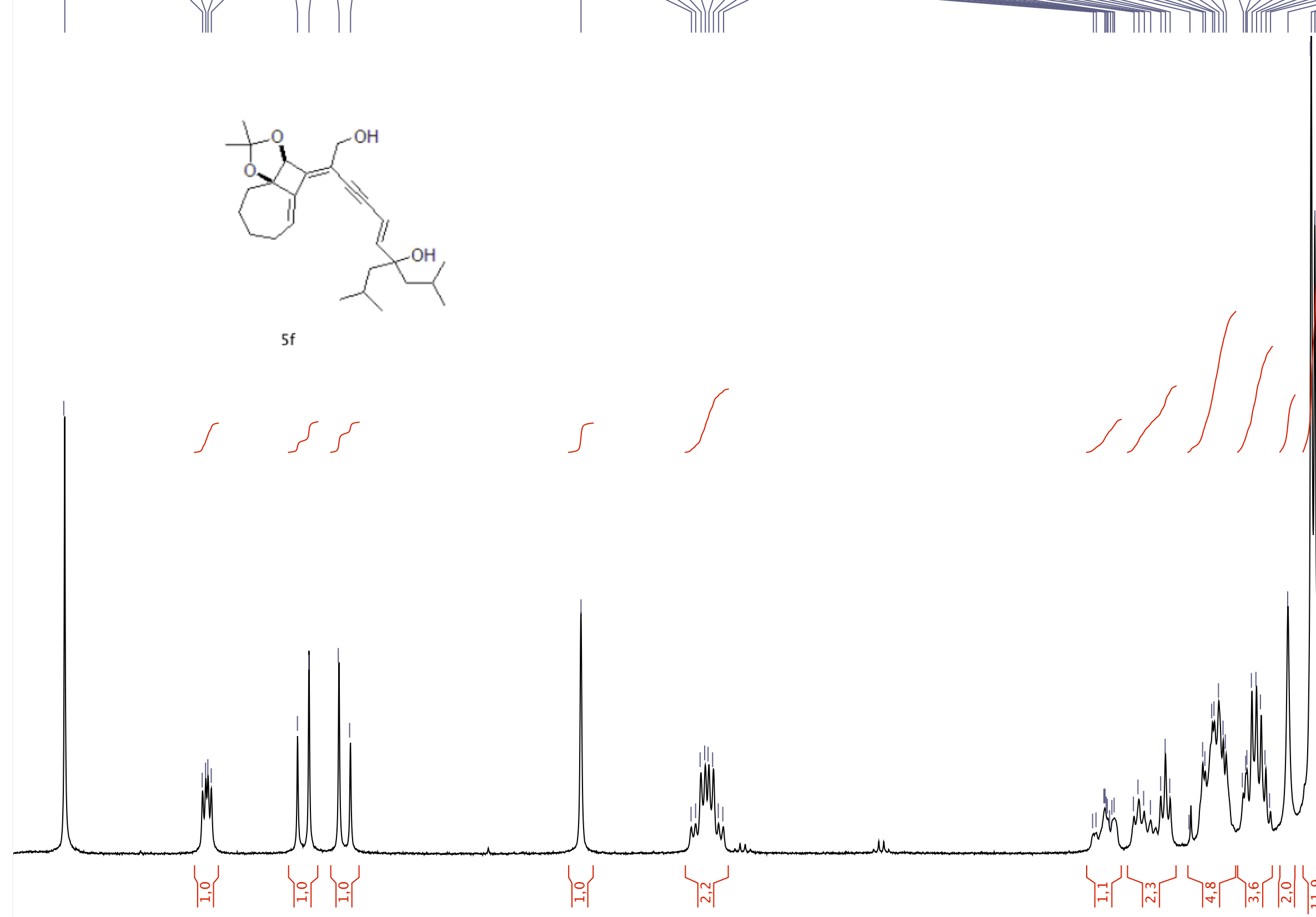


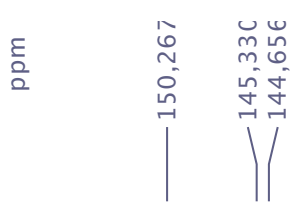

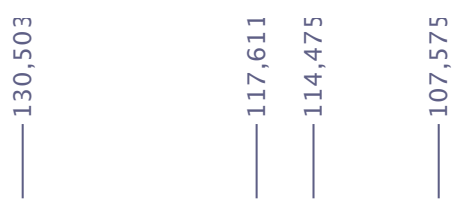

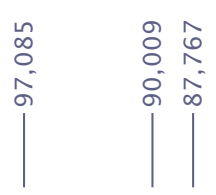

$\overbrace{\substack{0 \\ \tilde{0}}}^{\substack{\infty \\ 0}}$
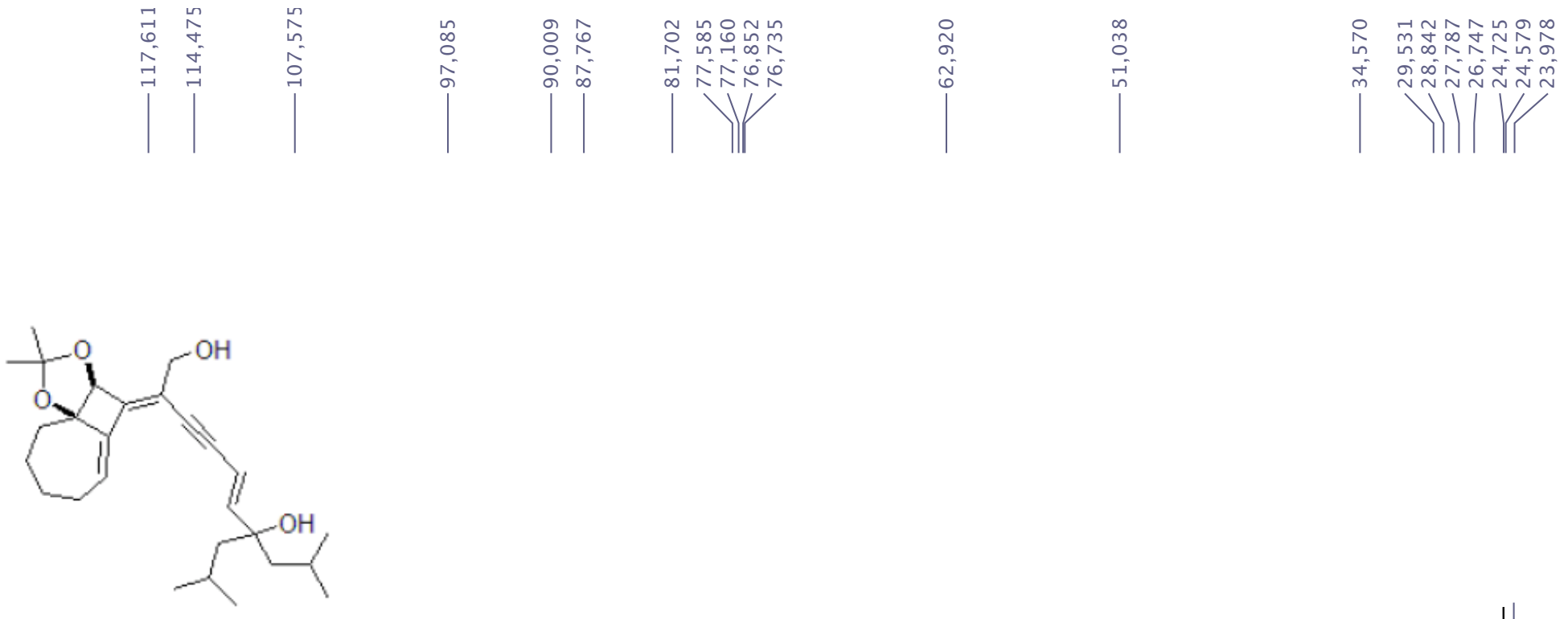

$5 f$

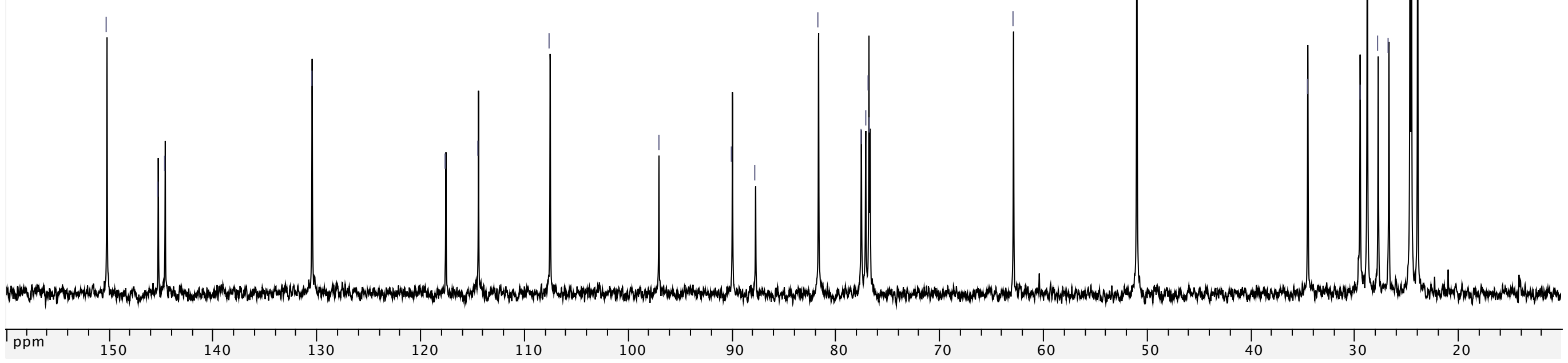




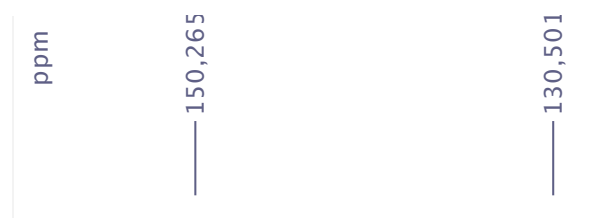

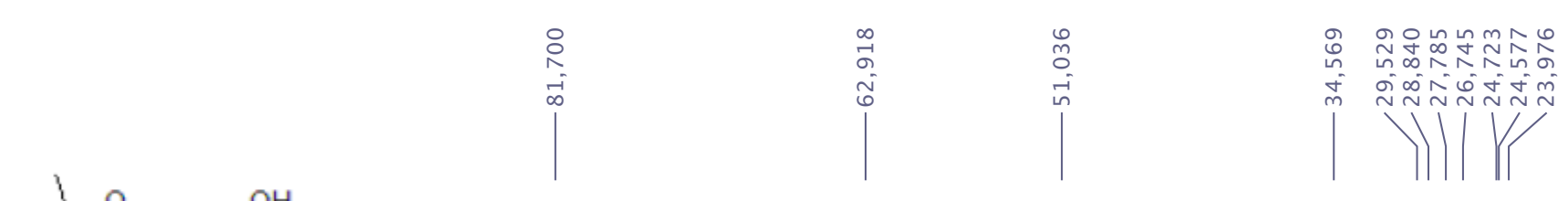

$5 f$

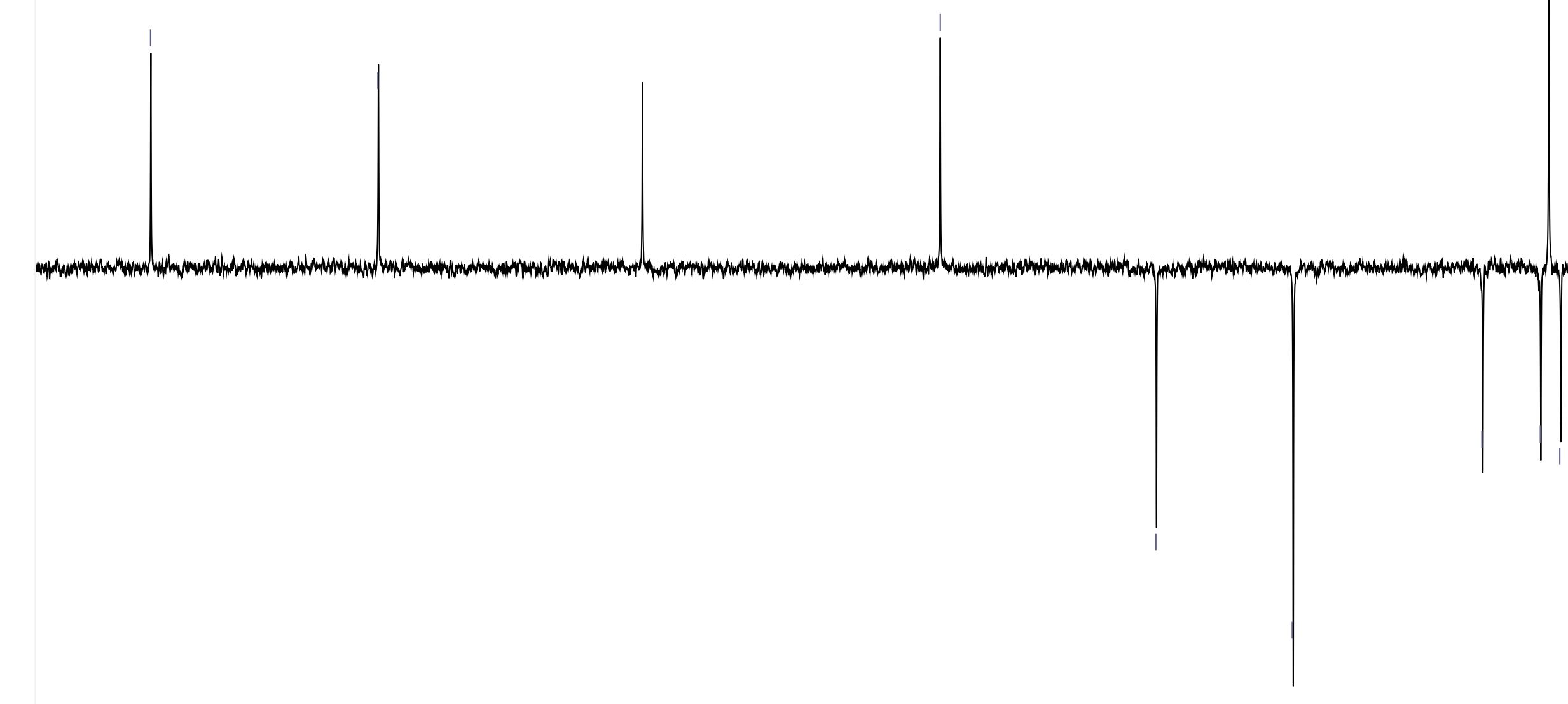




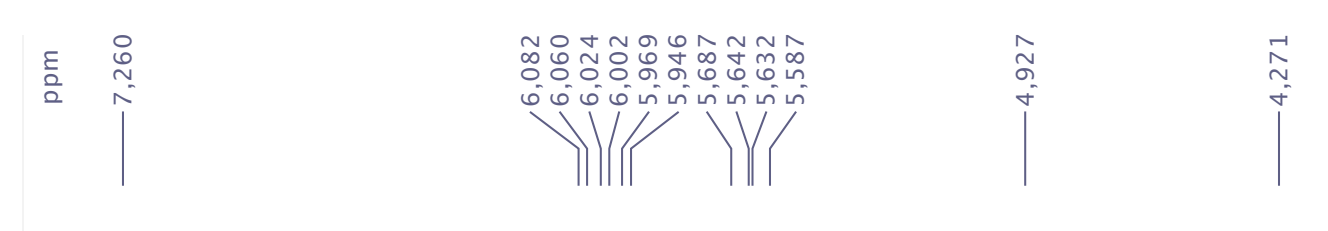

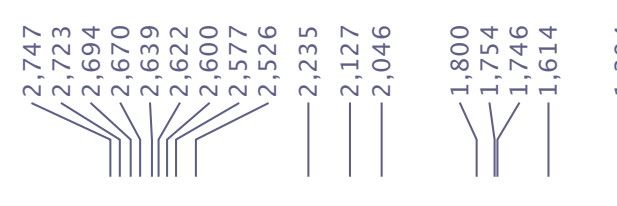
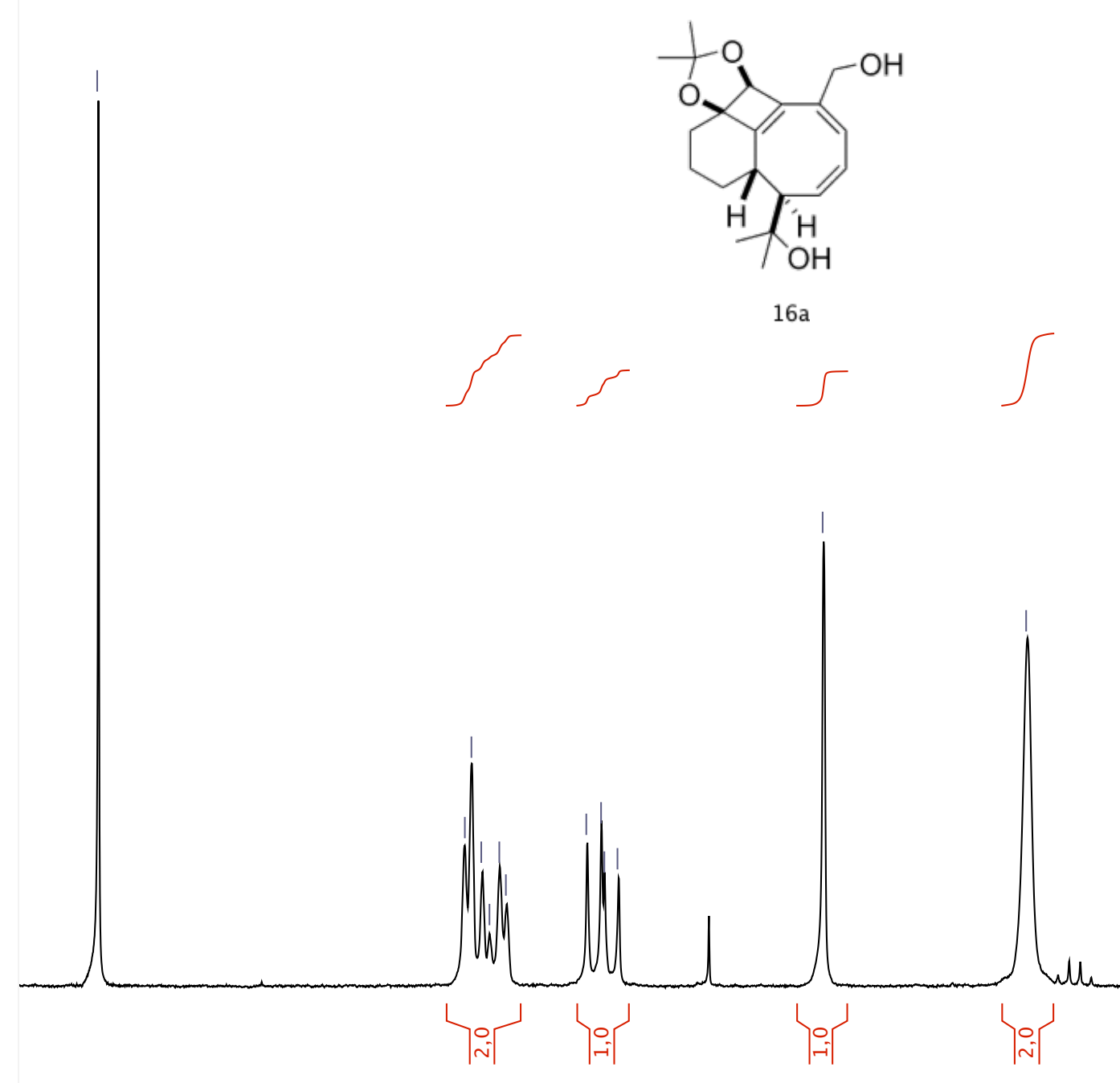


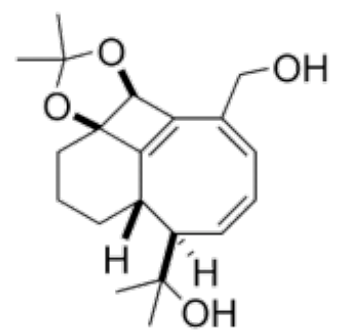

$16 a$

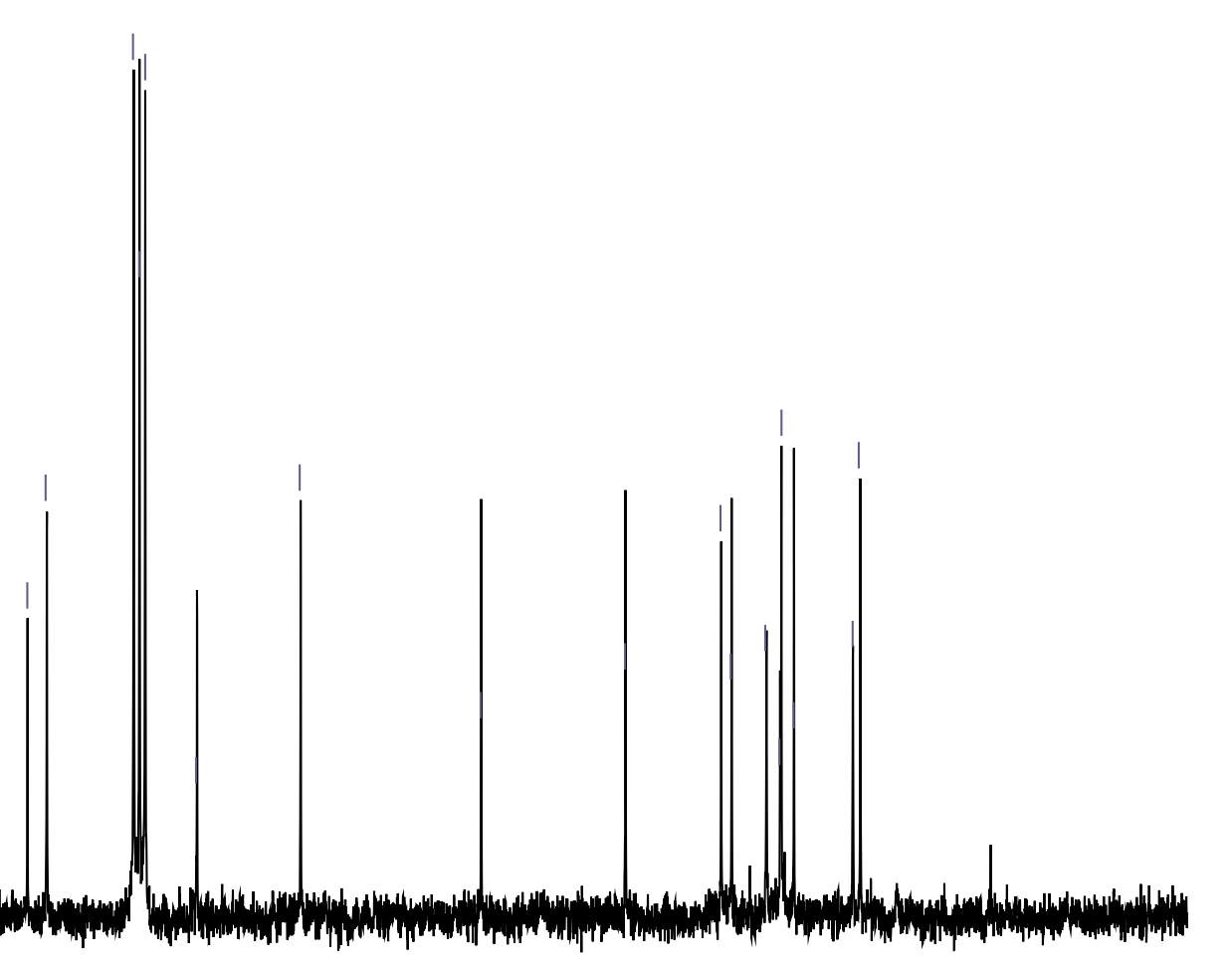


言
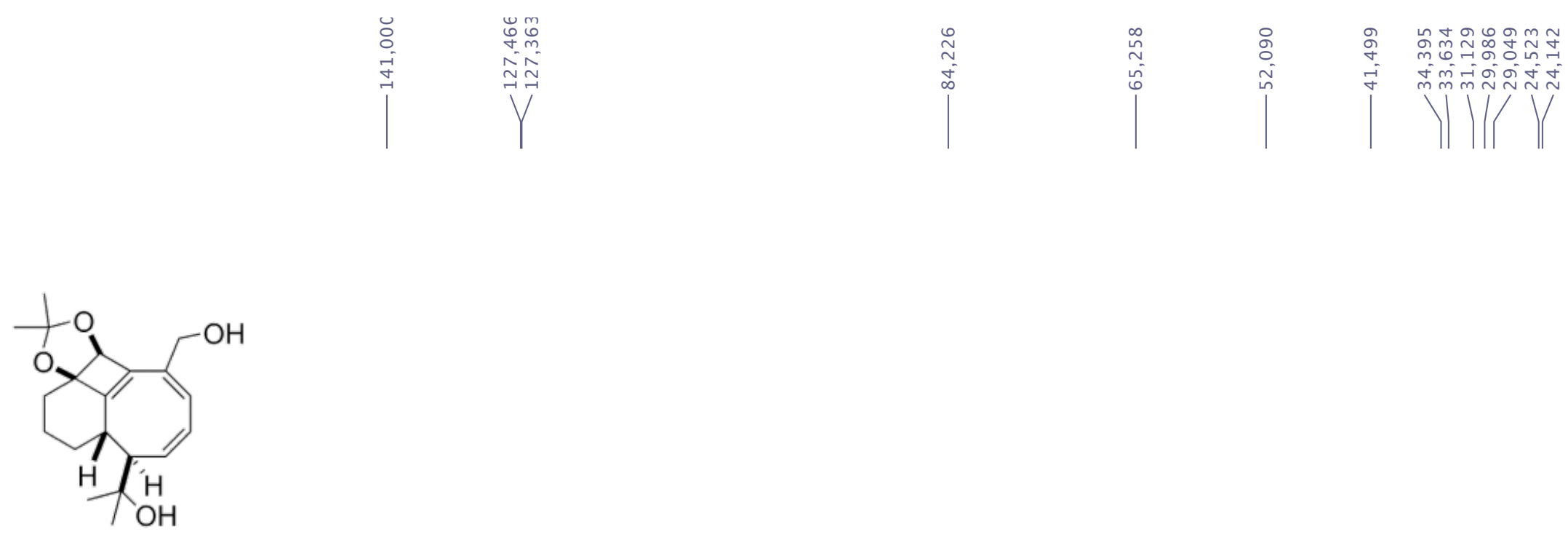

$16 \mathrm{a}$

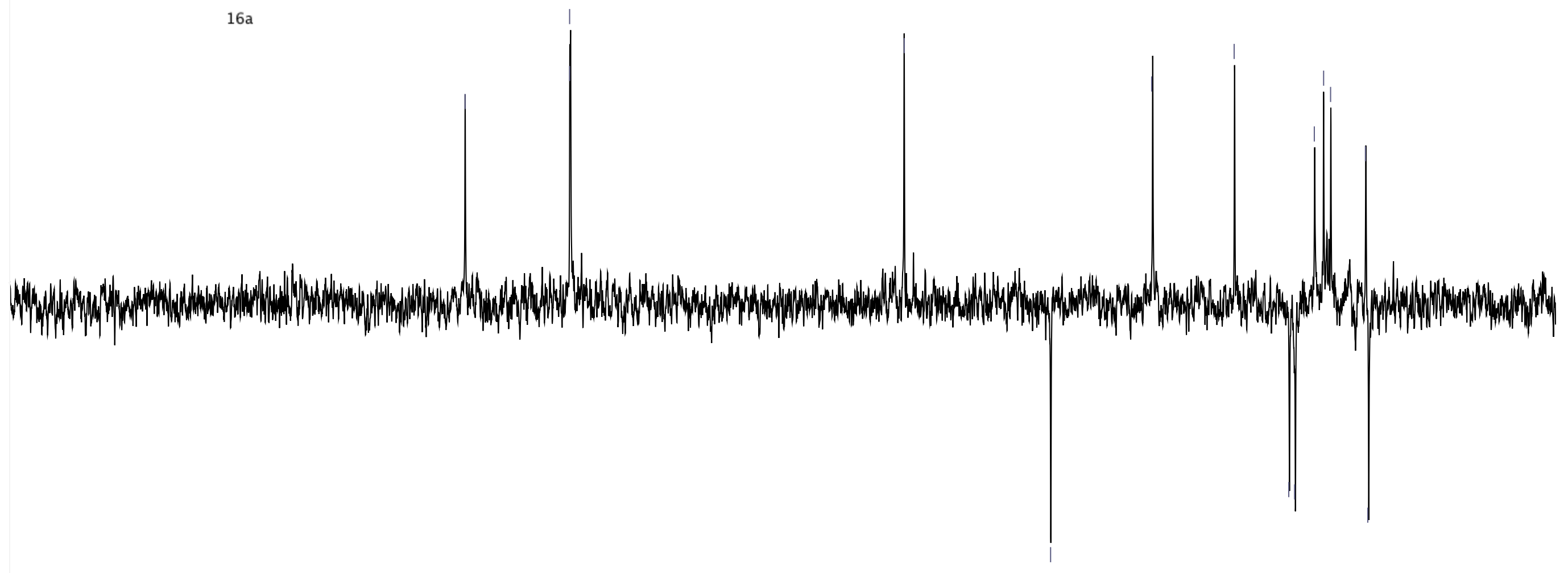

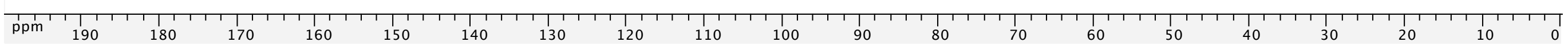




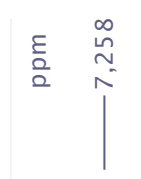

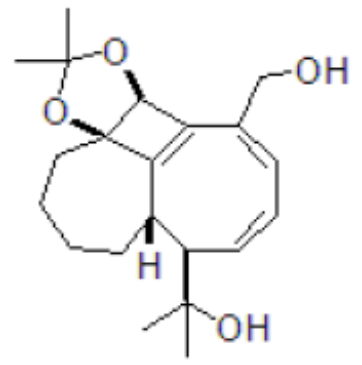

$18 \mathrm{a}$
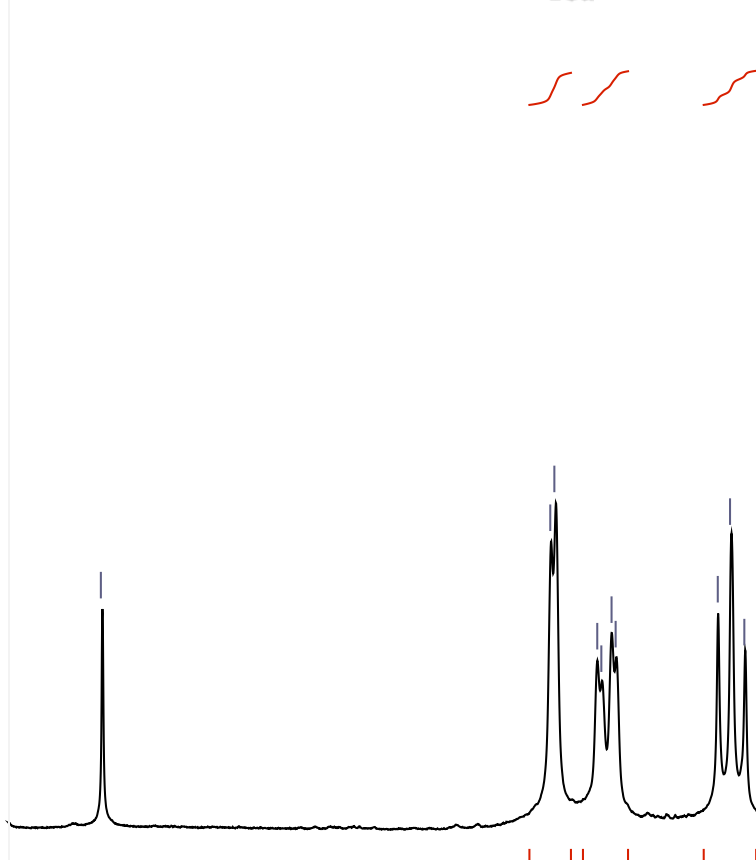

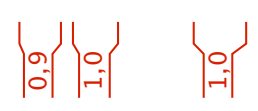
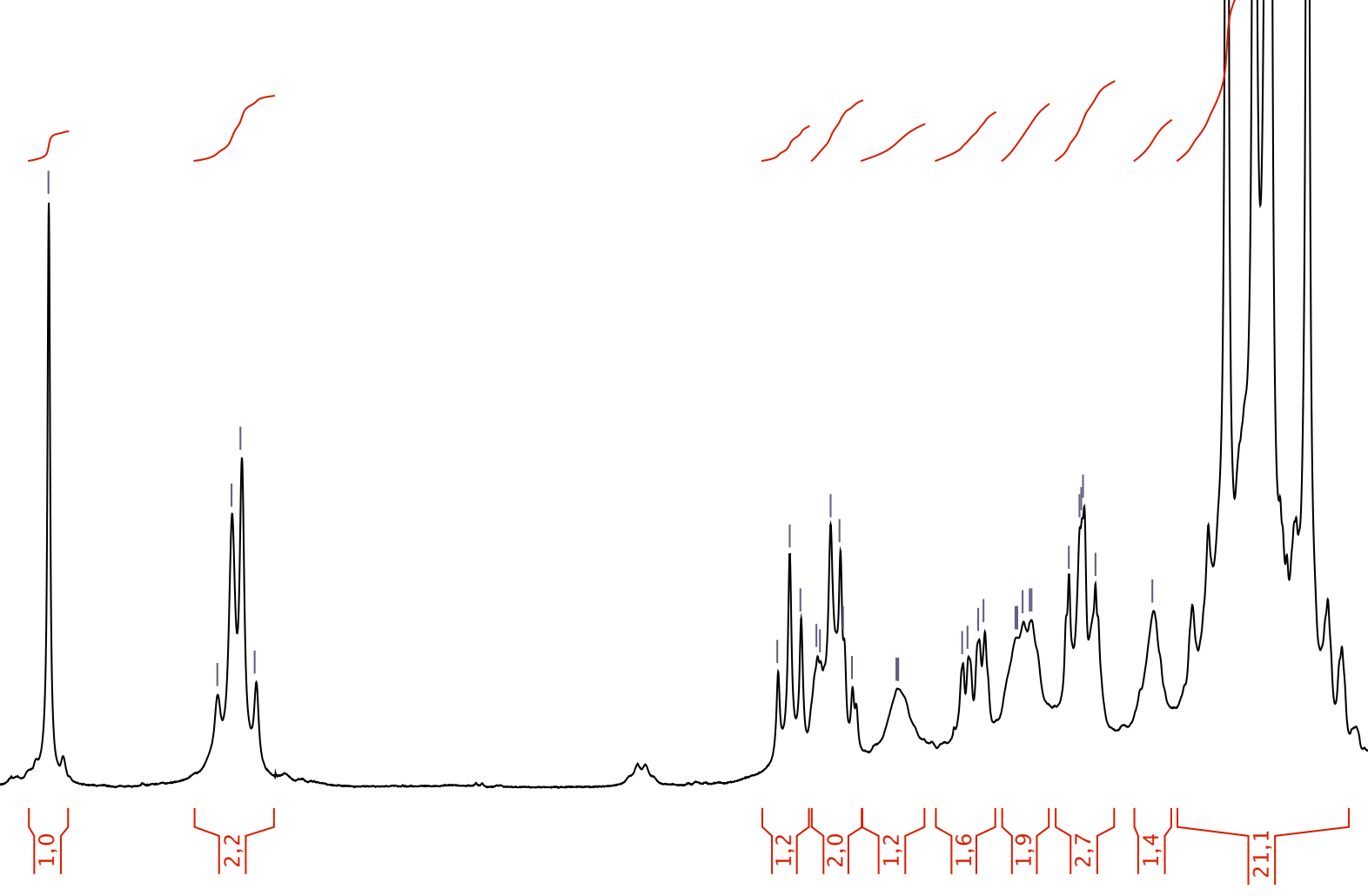


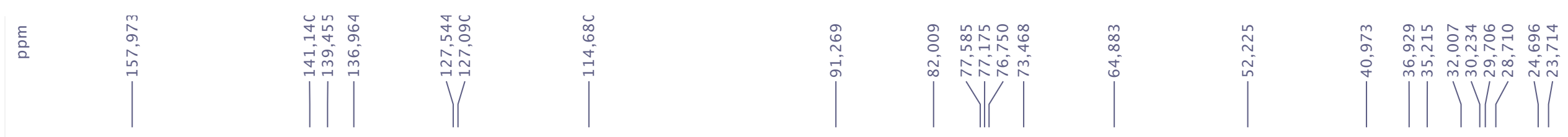

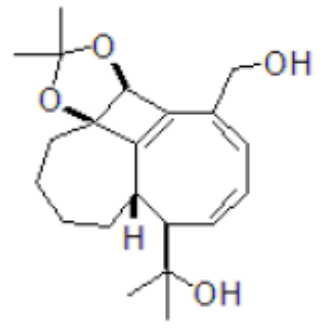

$18 a$

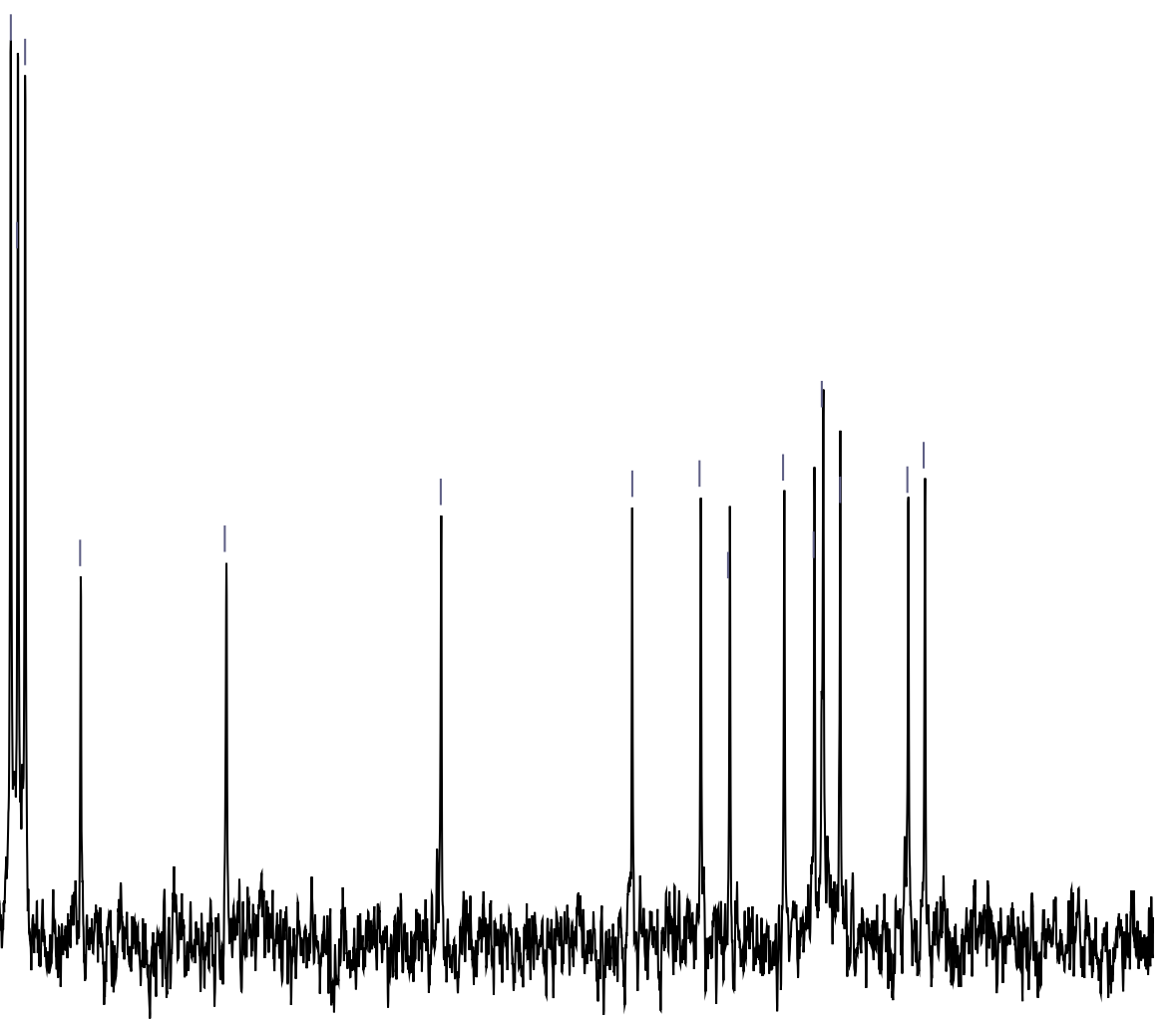

ppm I I

150

140

130

120

110

100

90

80

70

60

50

40

30 

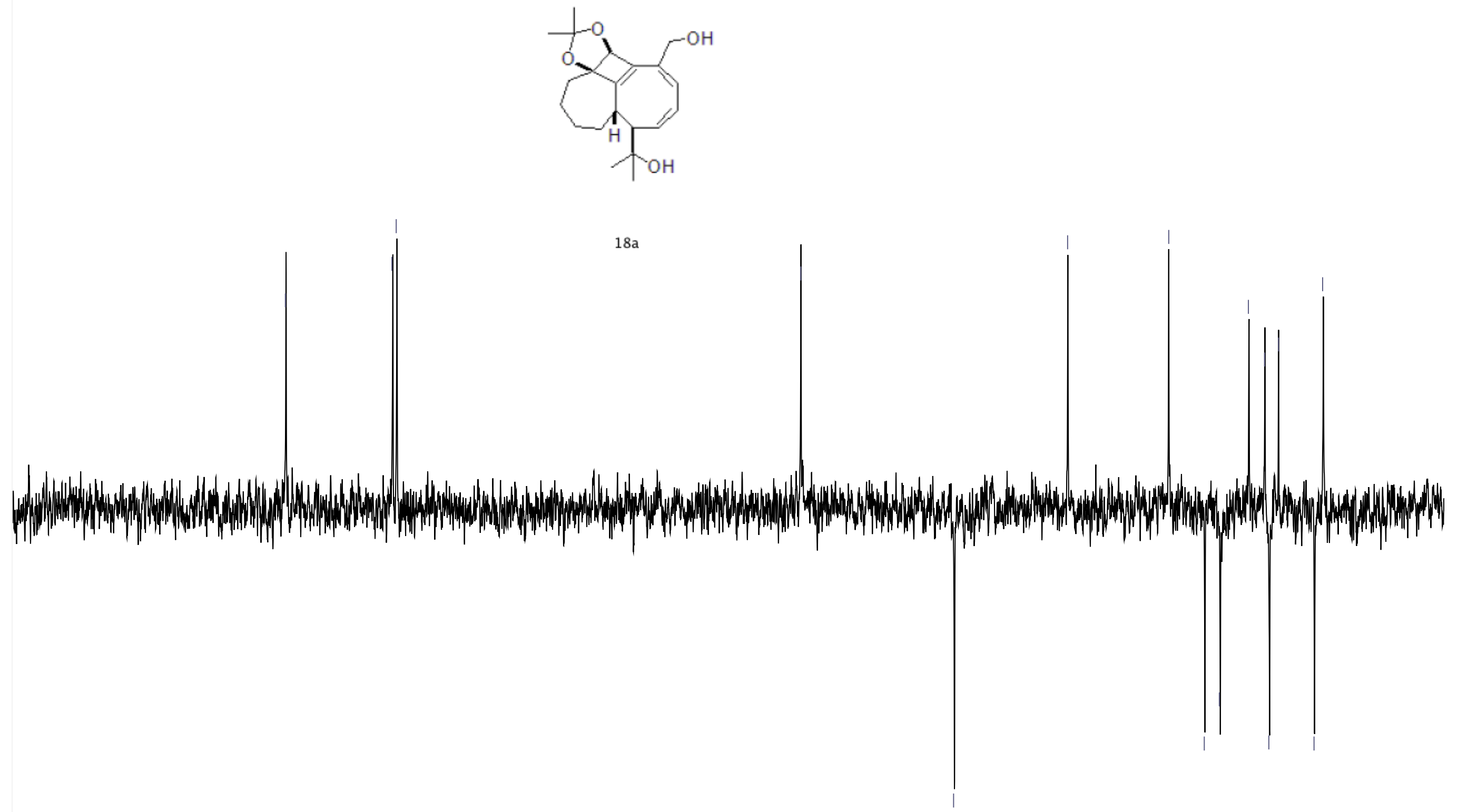


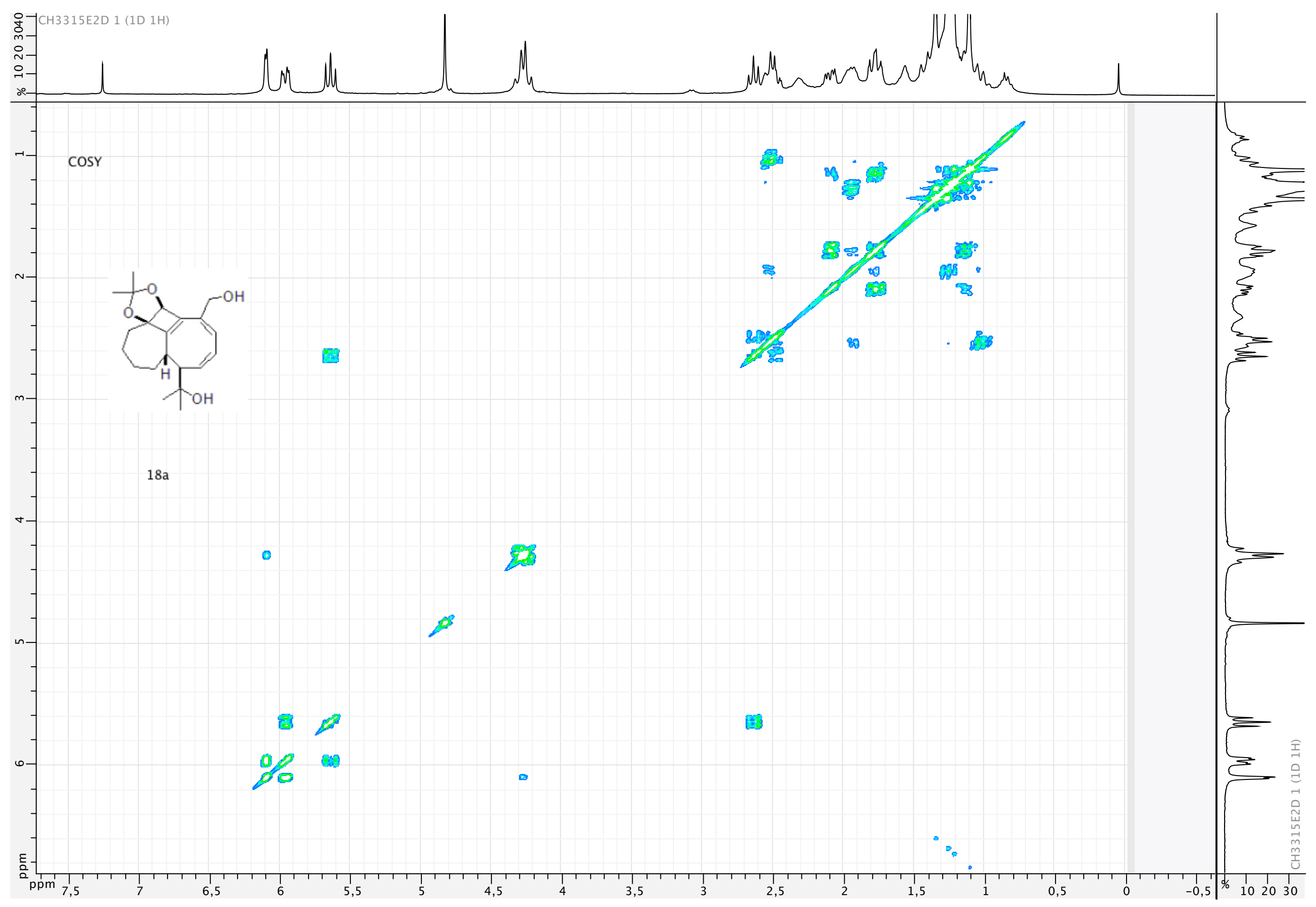




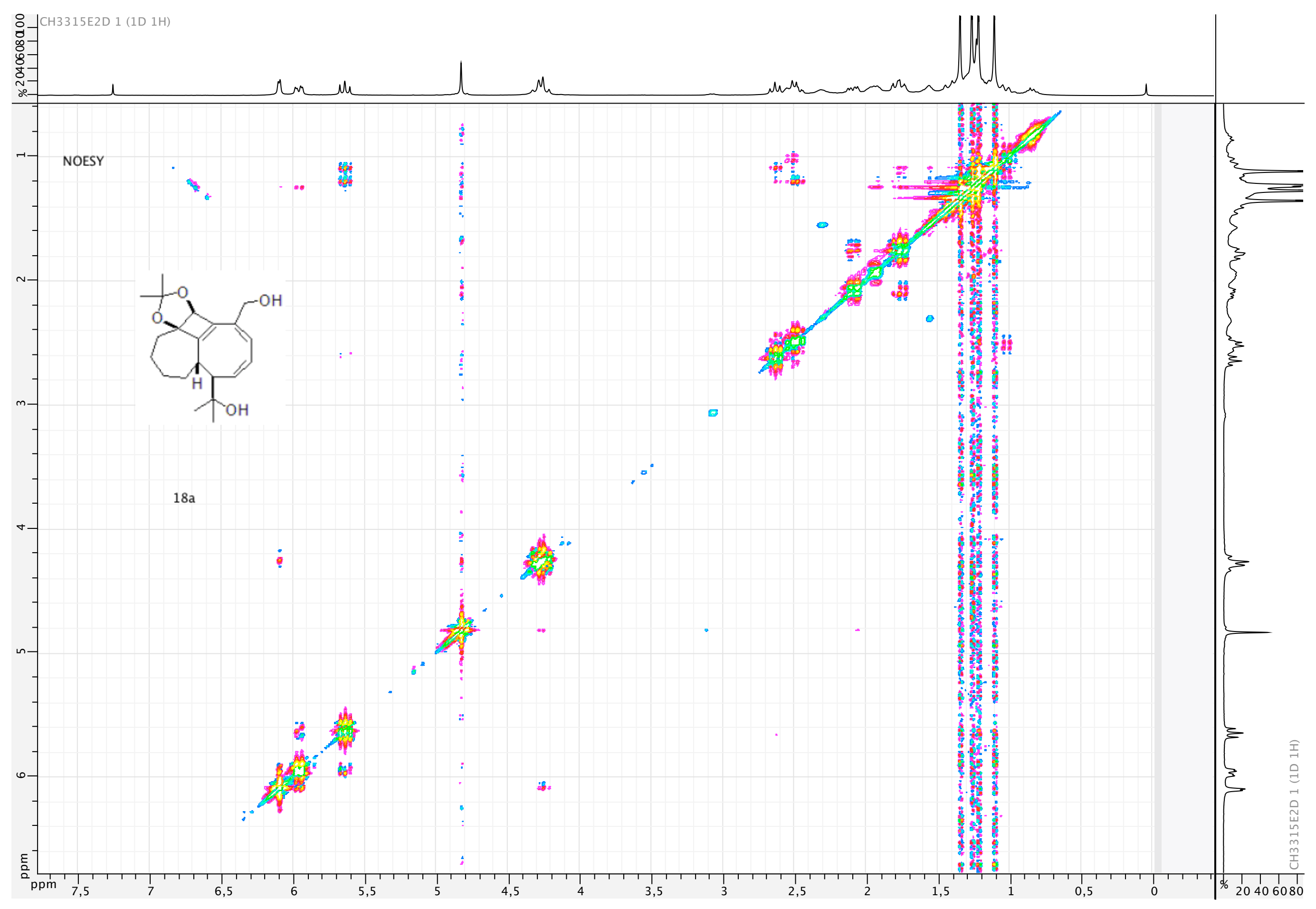




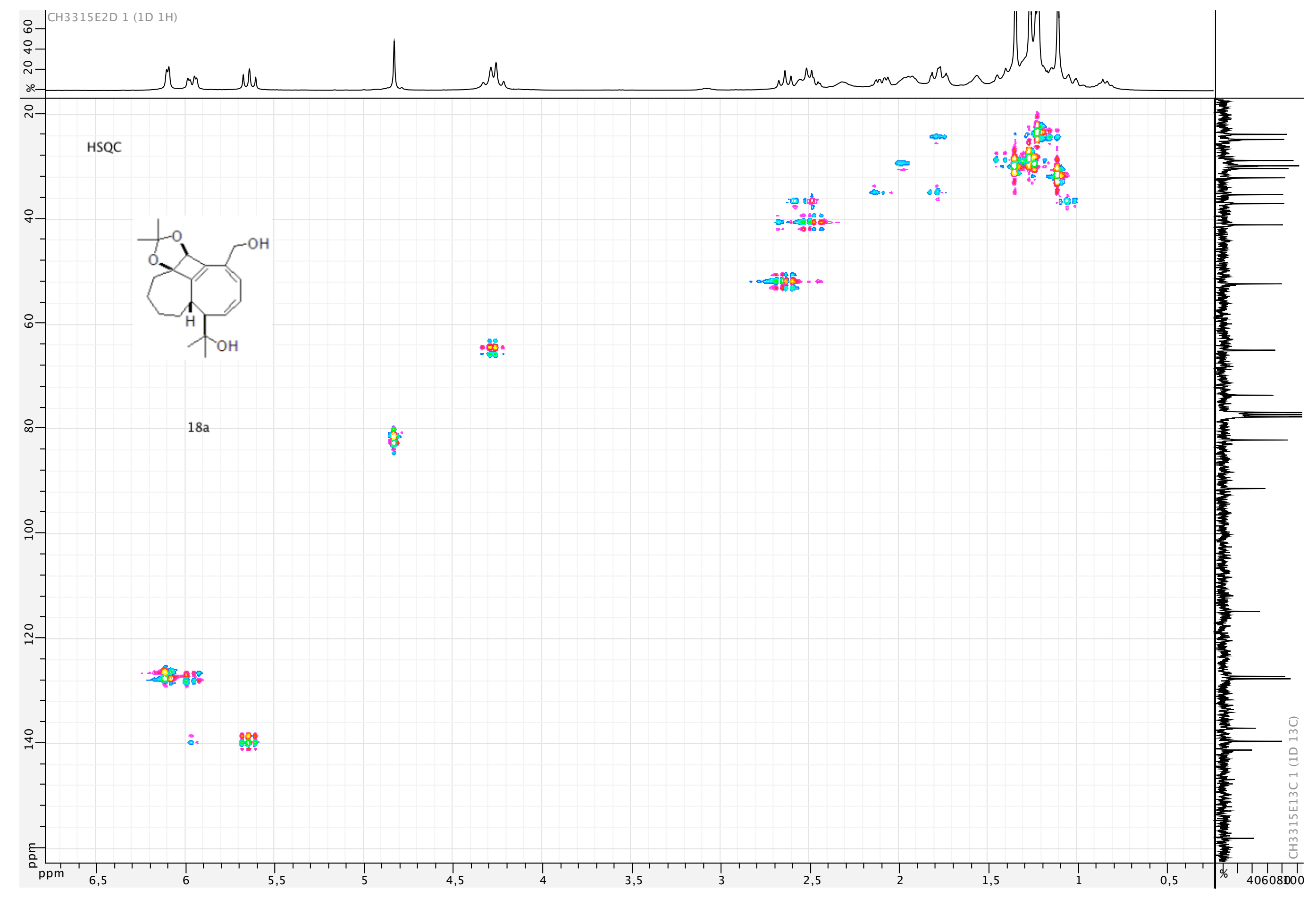




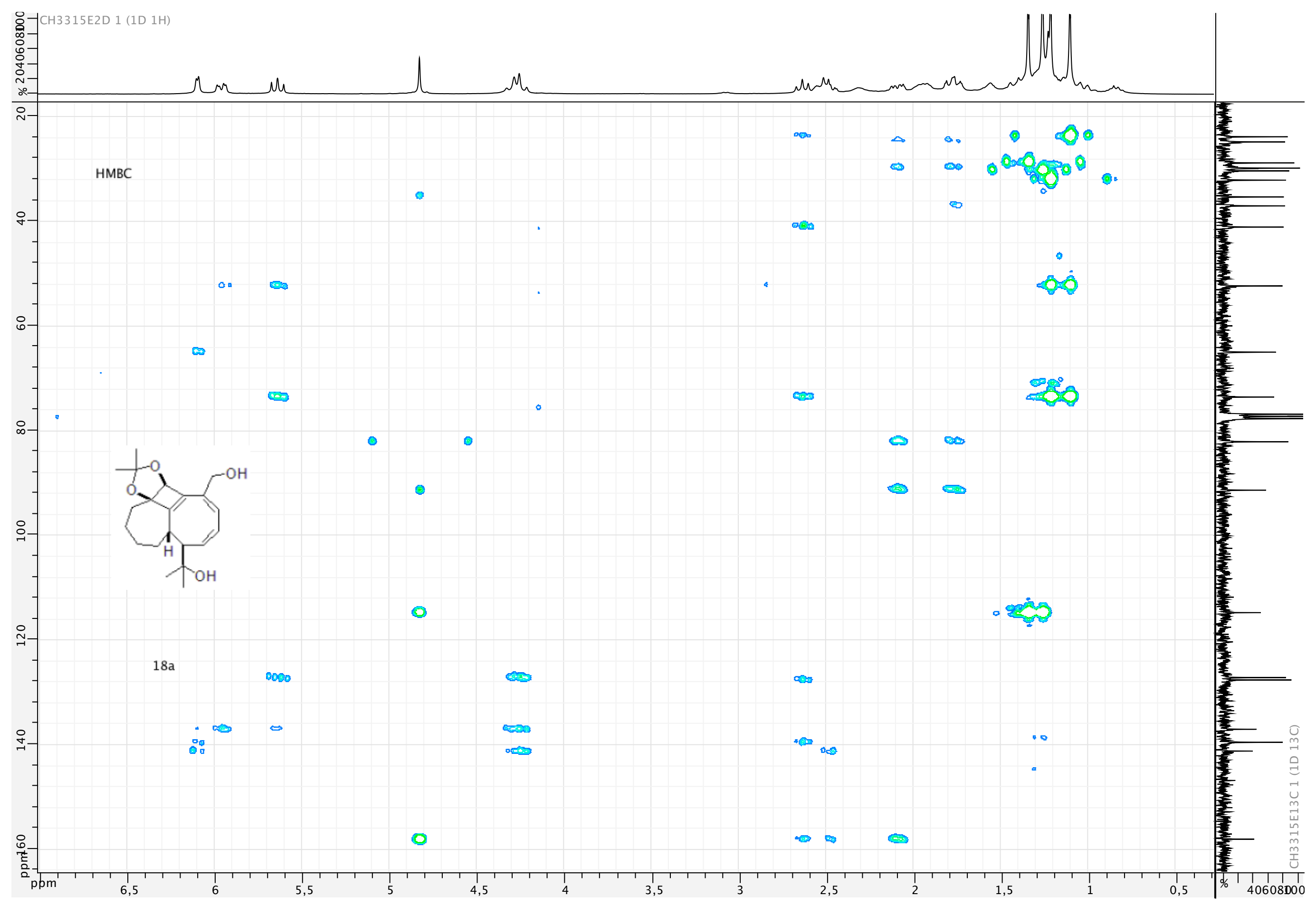




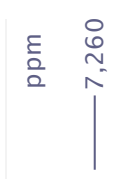

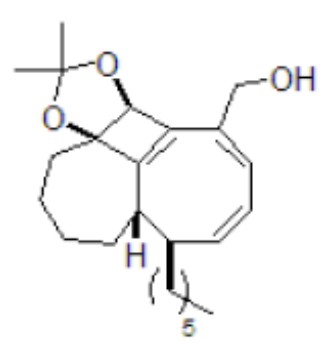

$18 \mathrm{~b}$

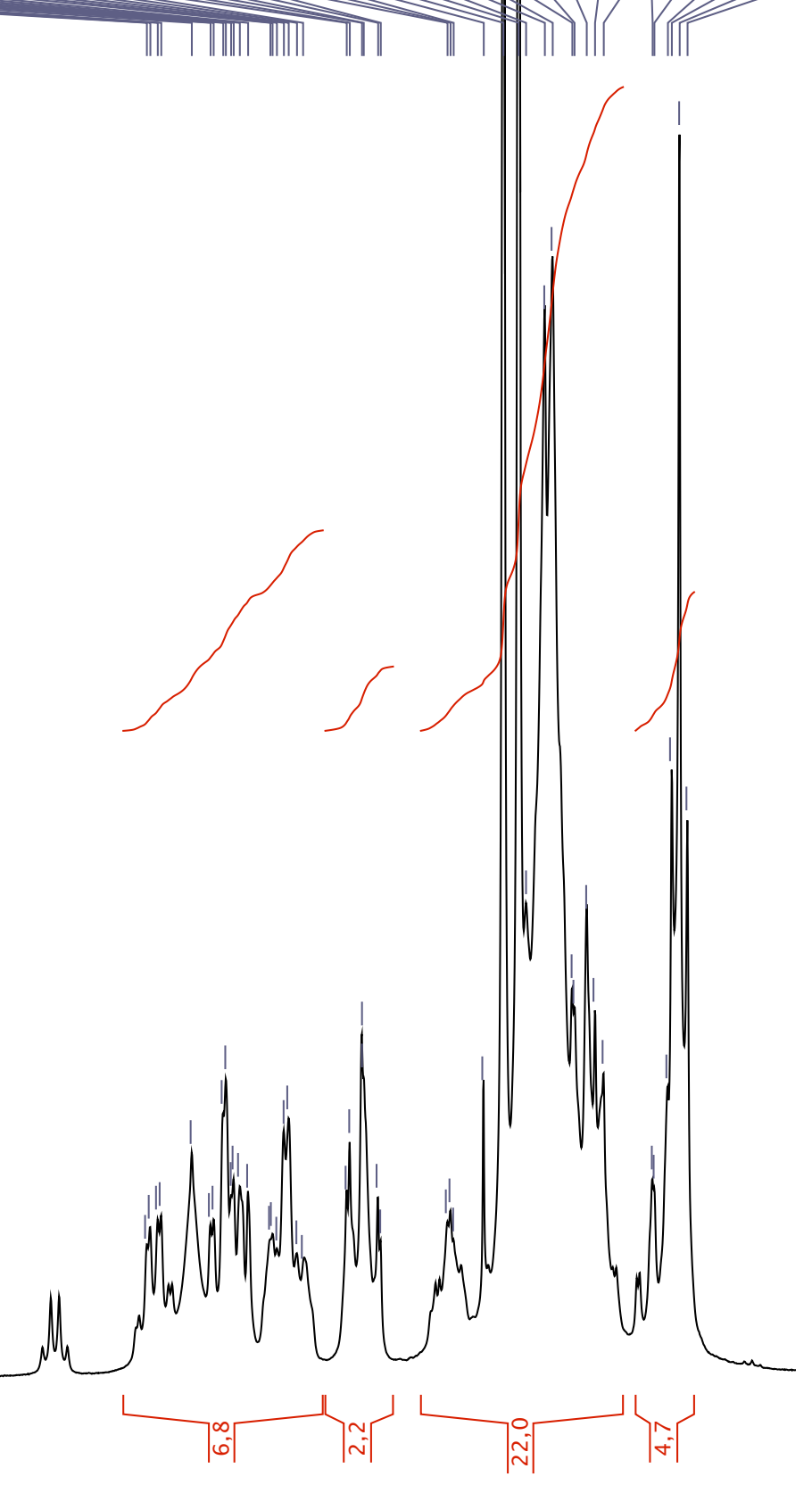




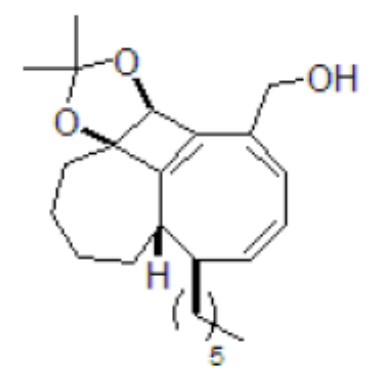

$18 \mathrm{~b}$

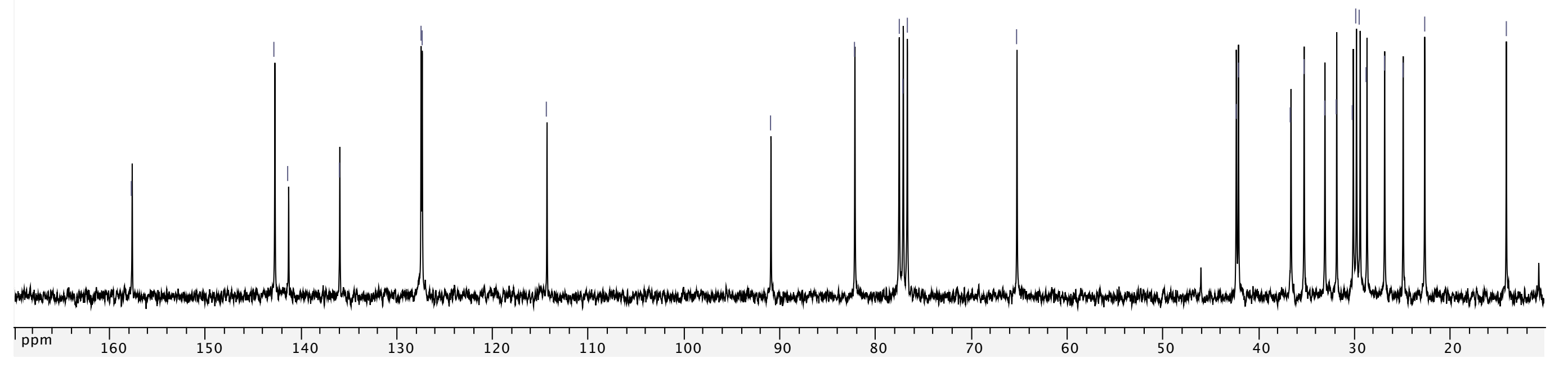




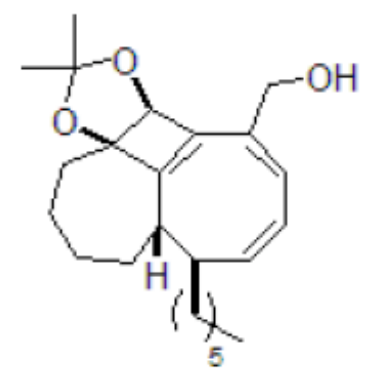

$18 b$

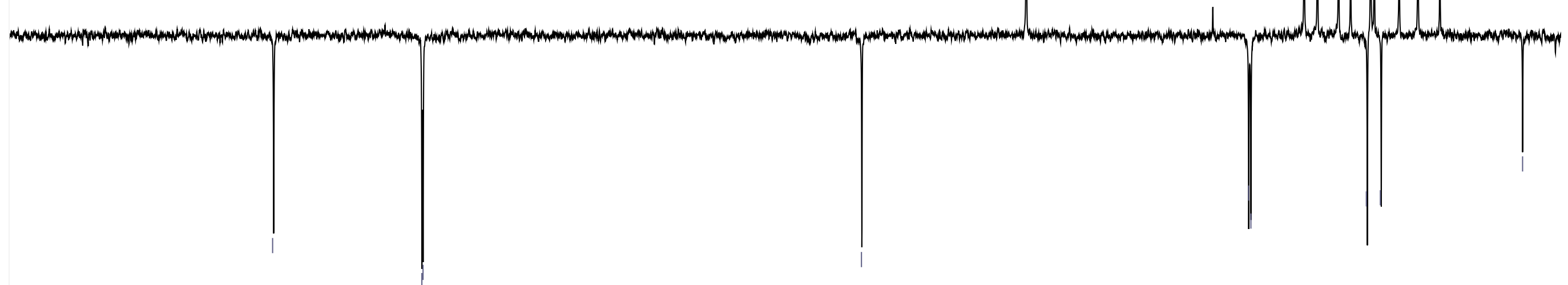




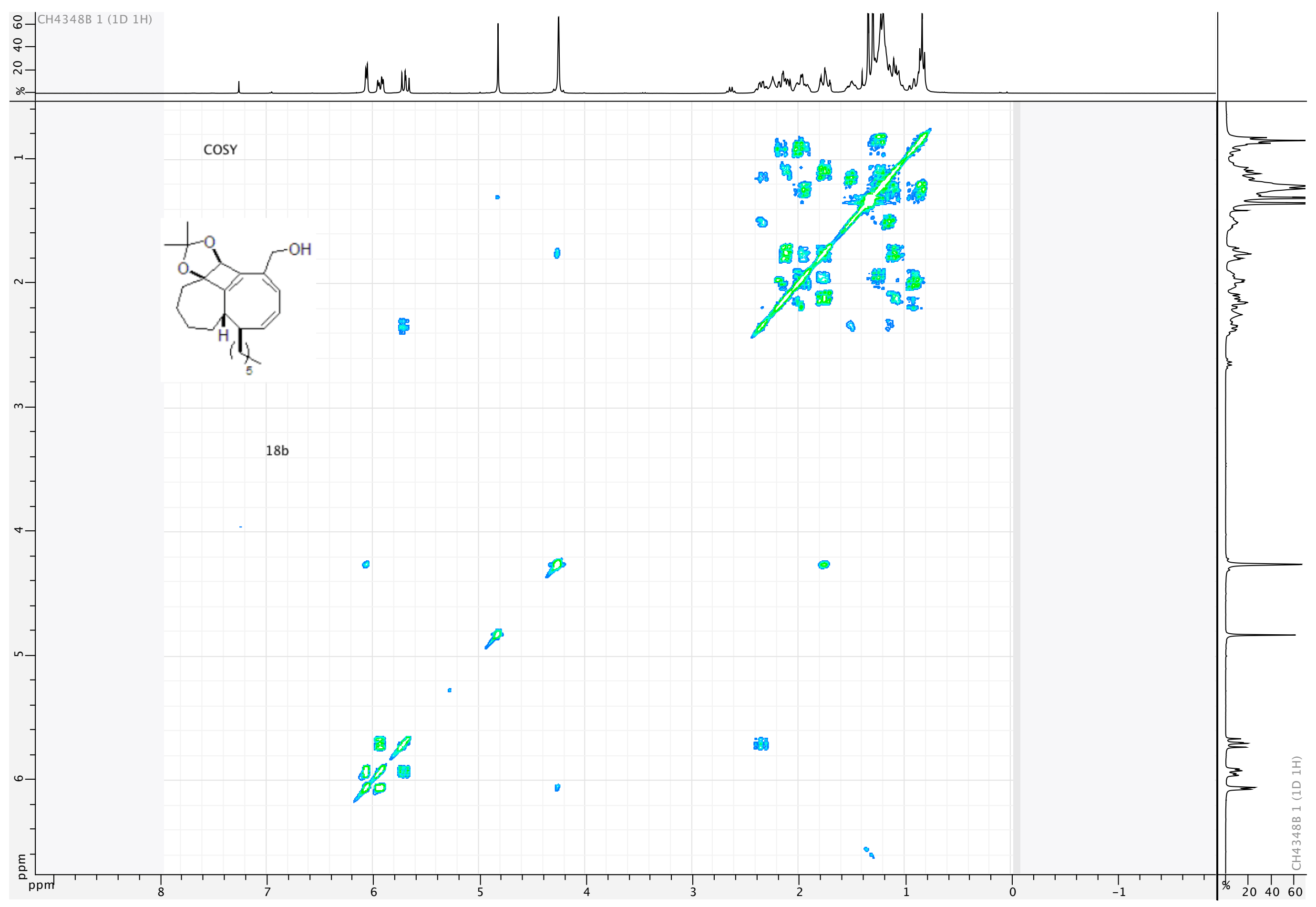




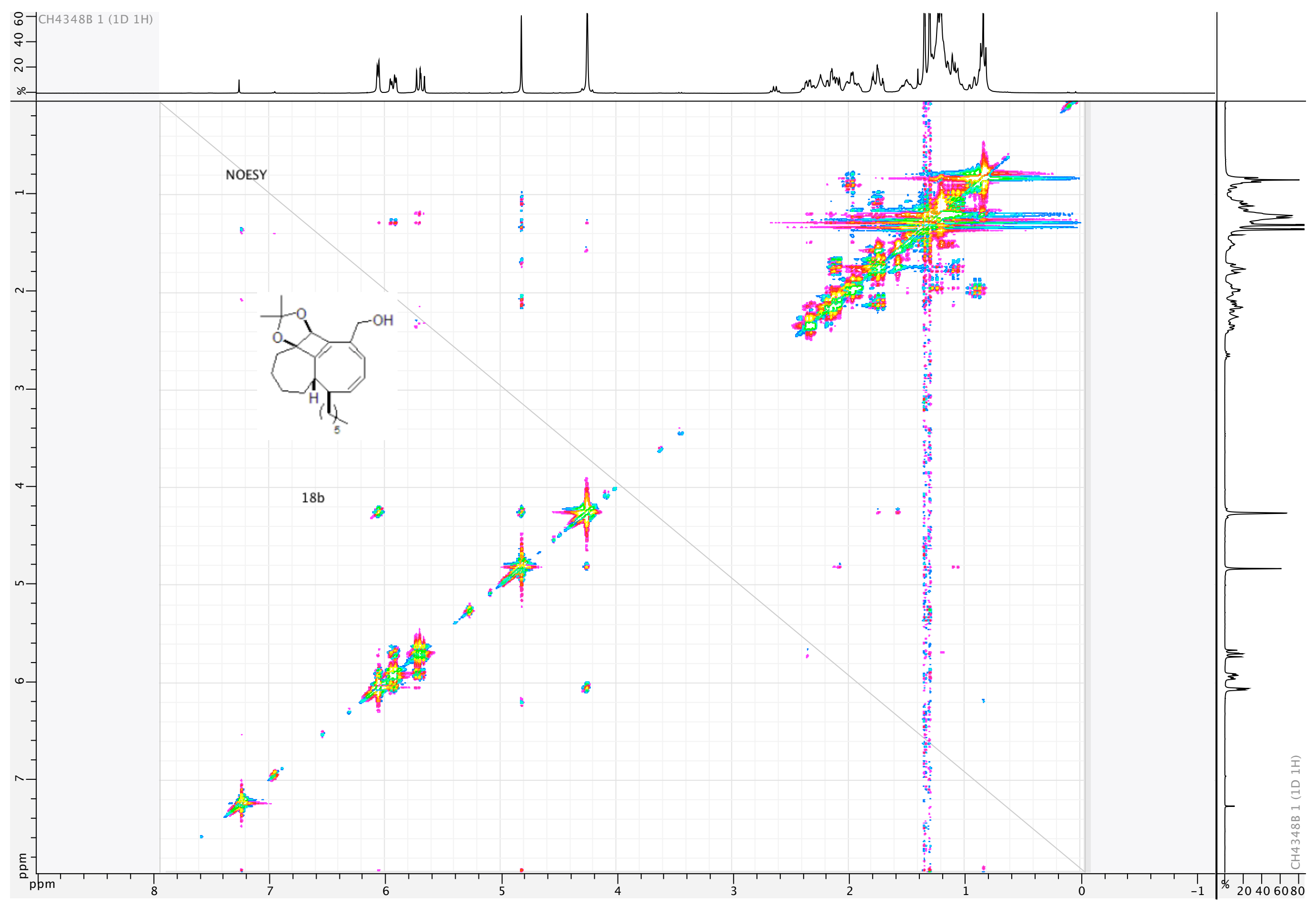




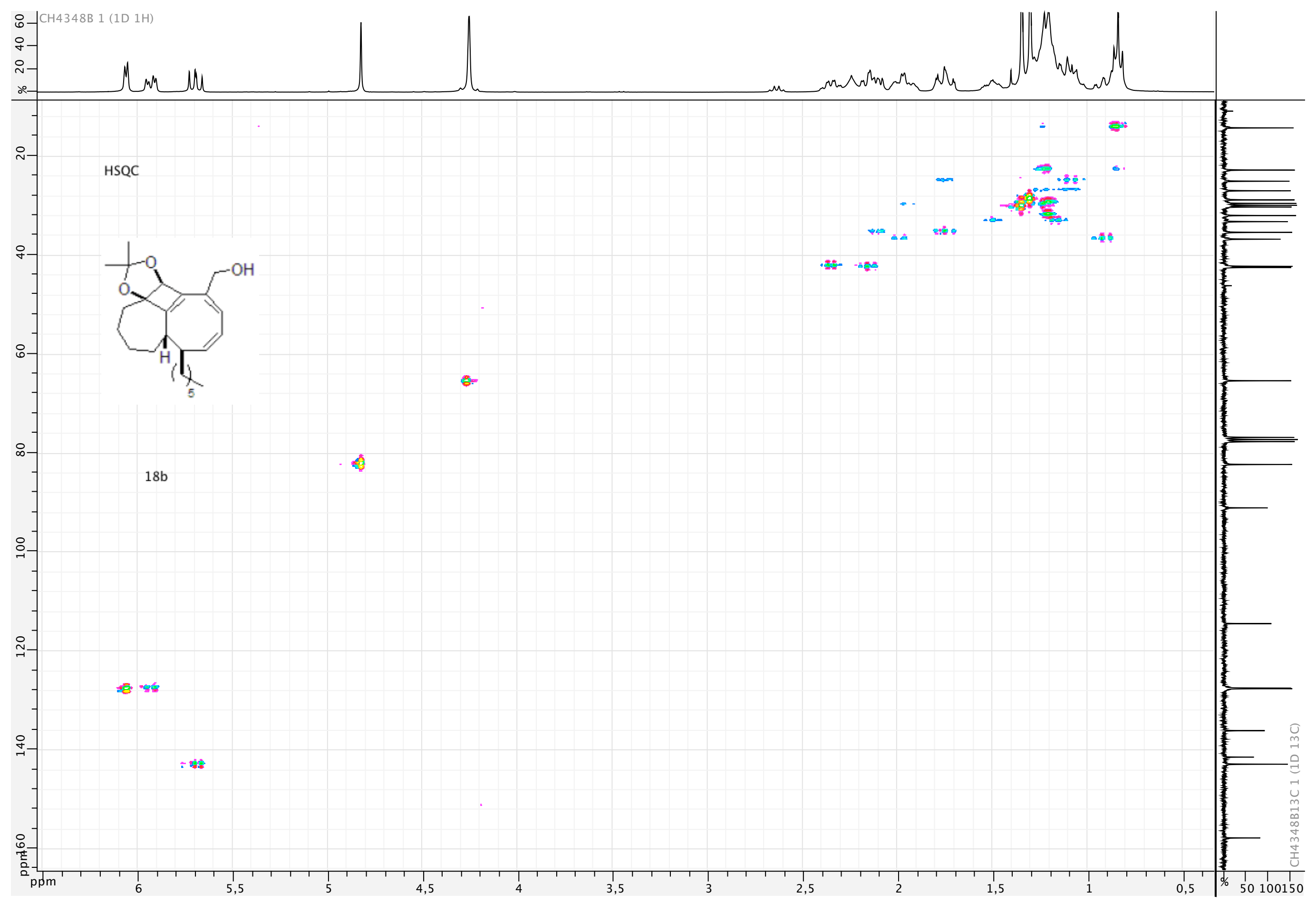




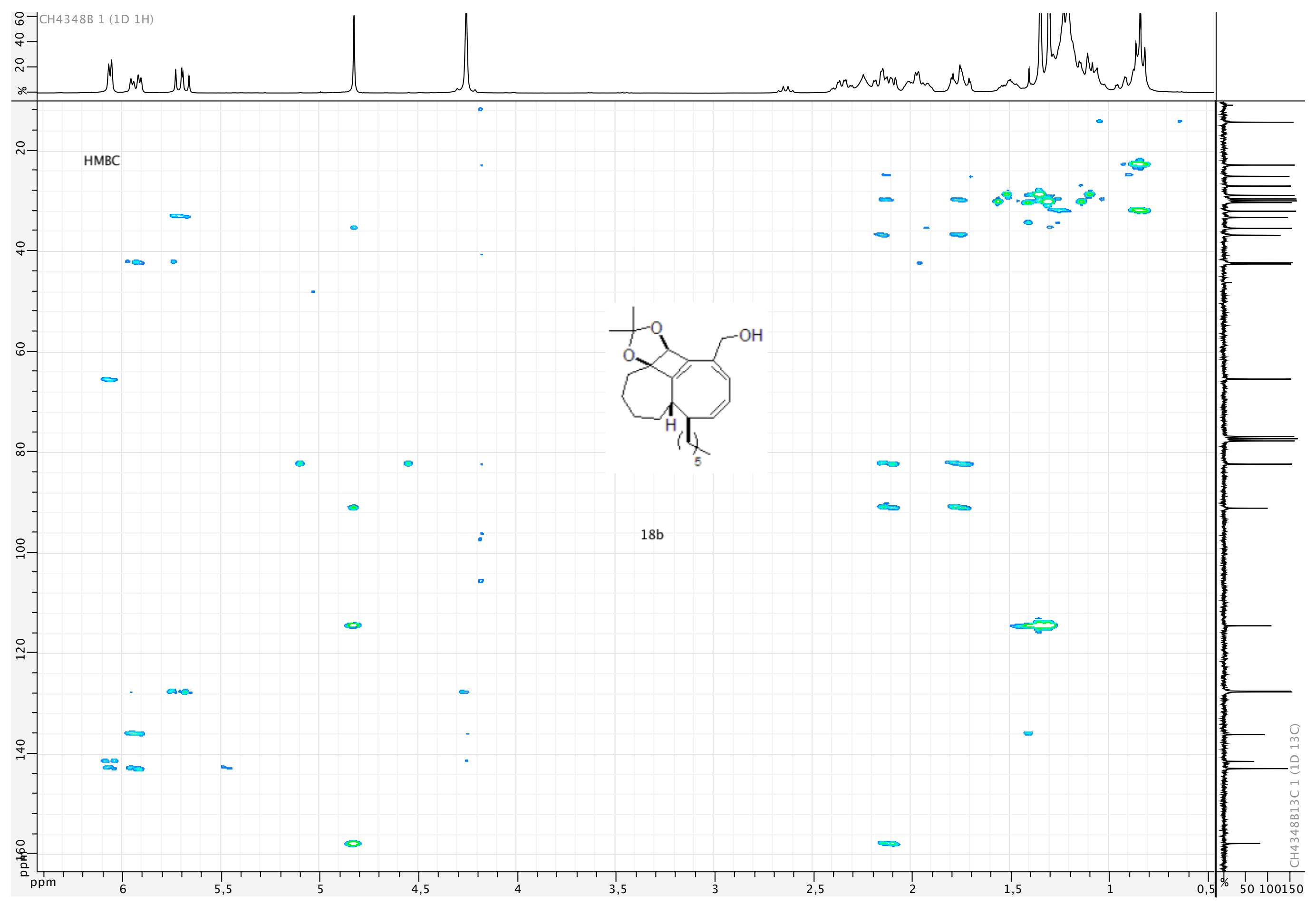



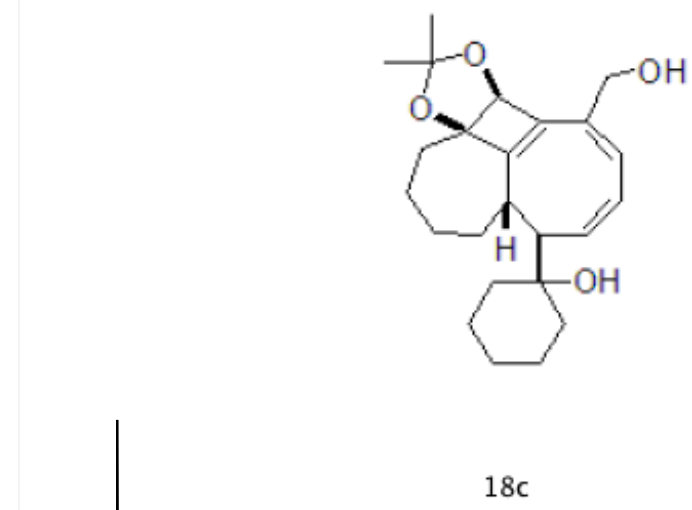

$18 \mathrm{c}$
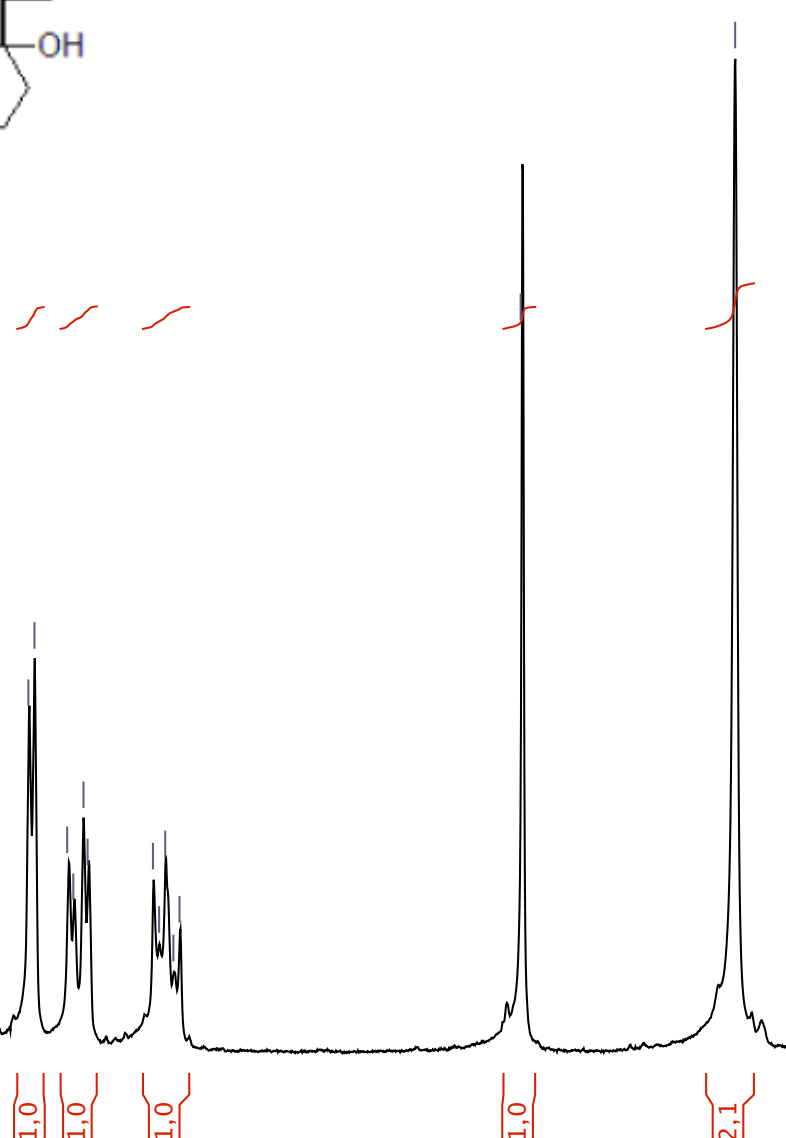
|

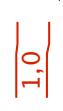




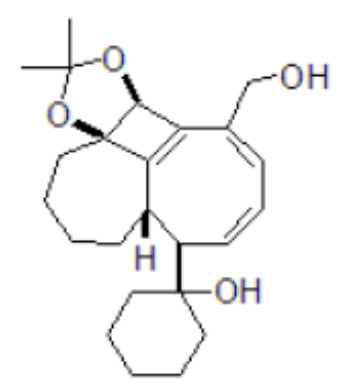

$18 \mathrm{c}$

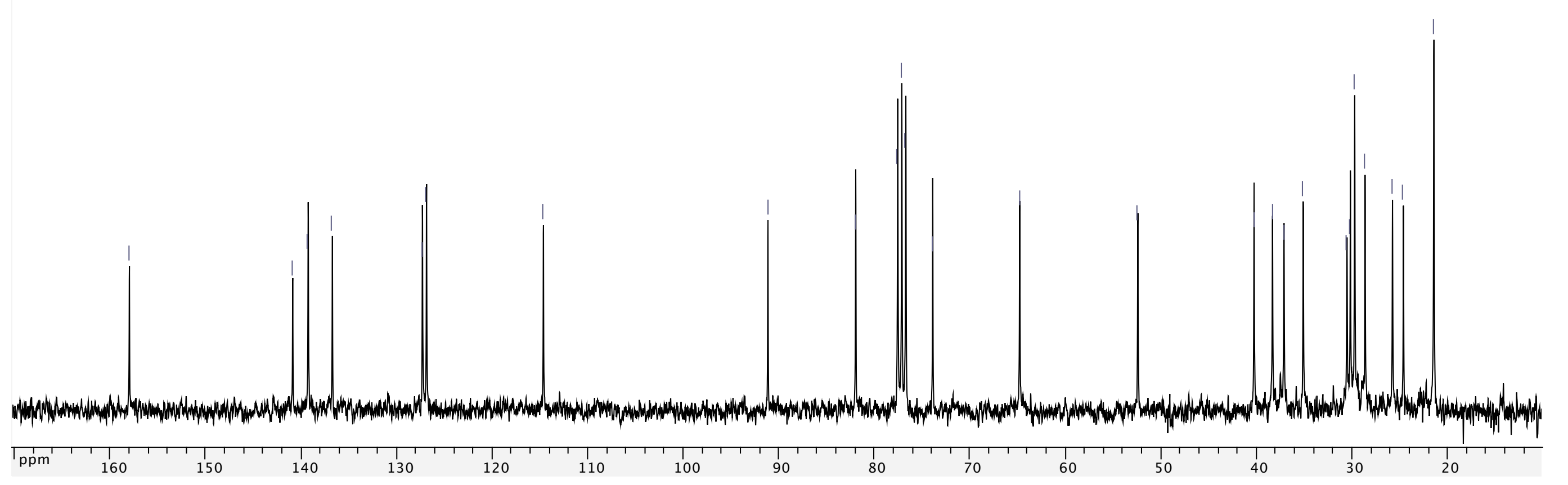



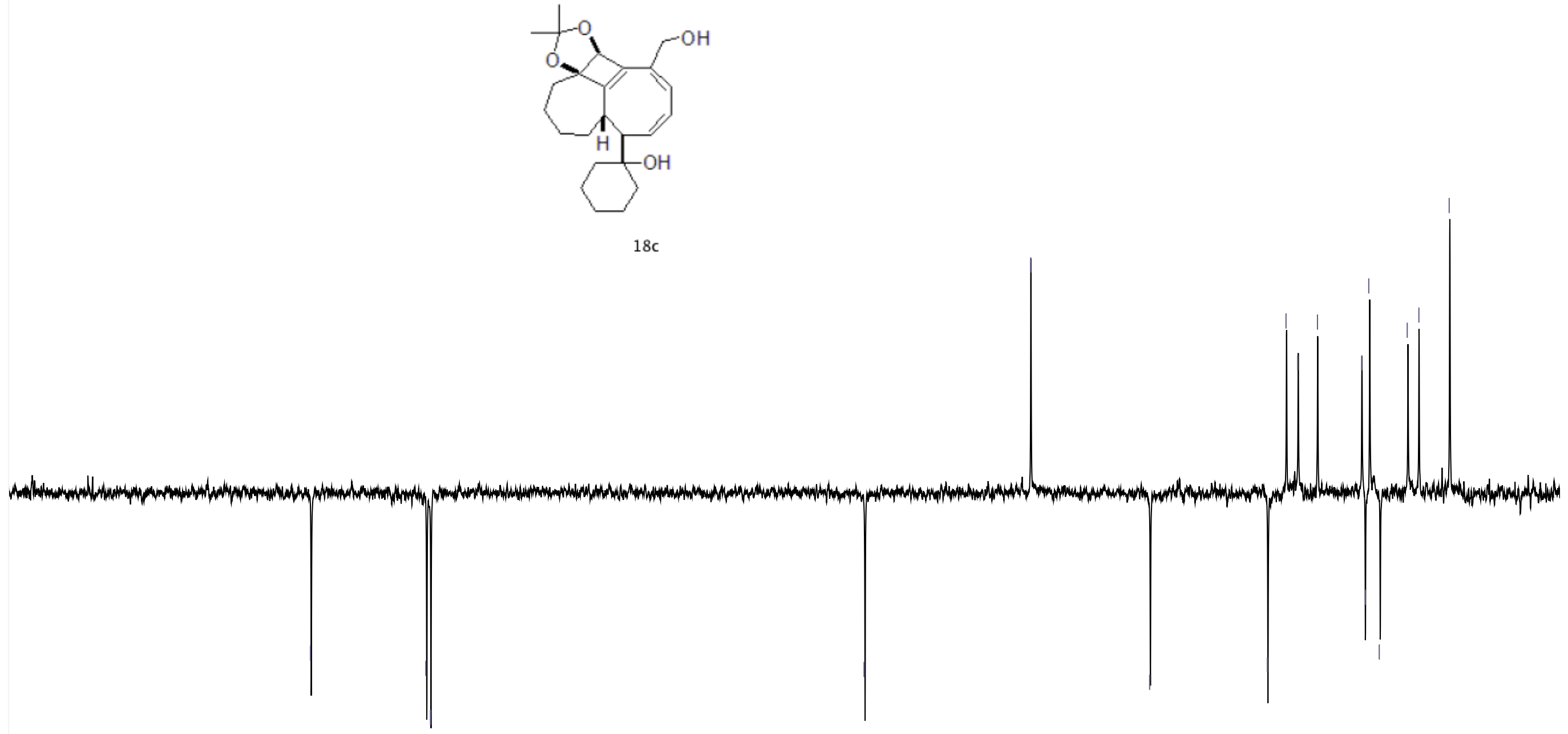


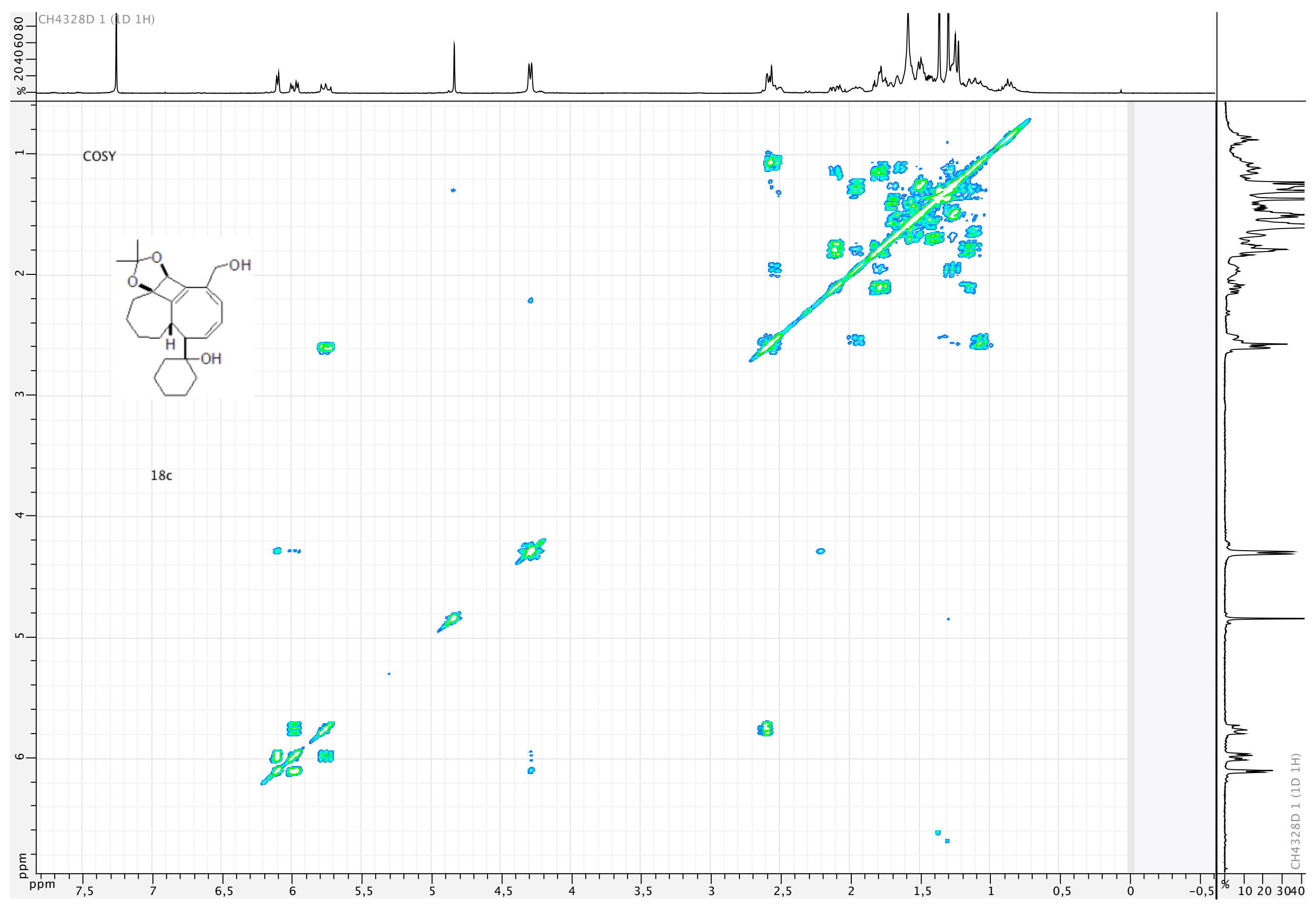




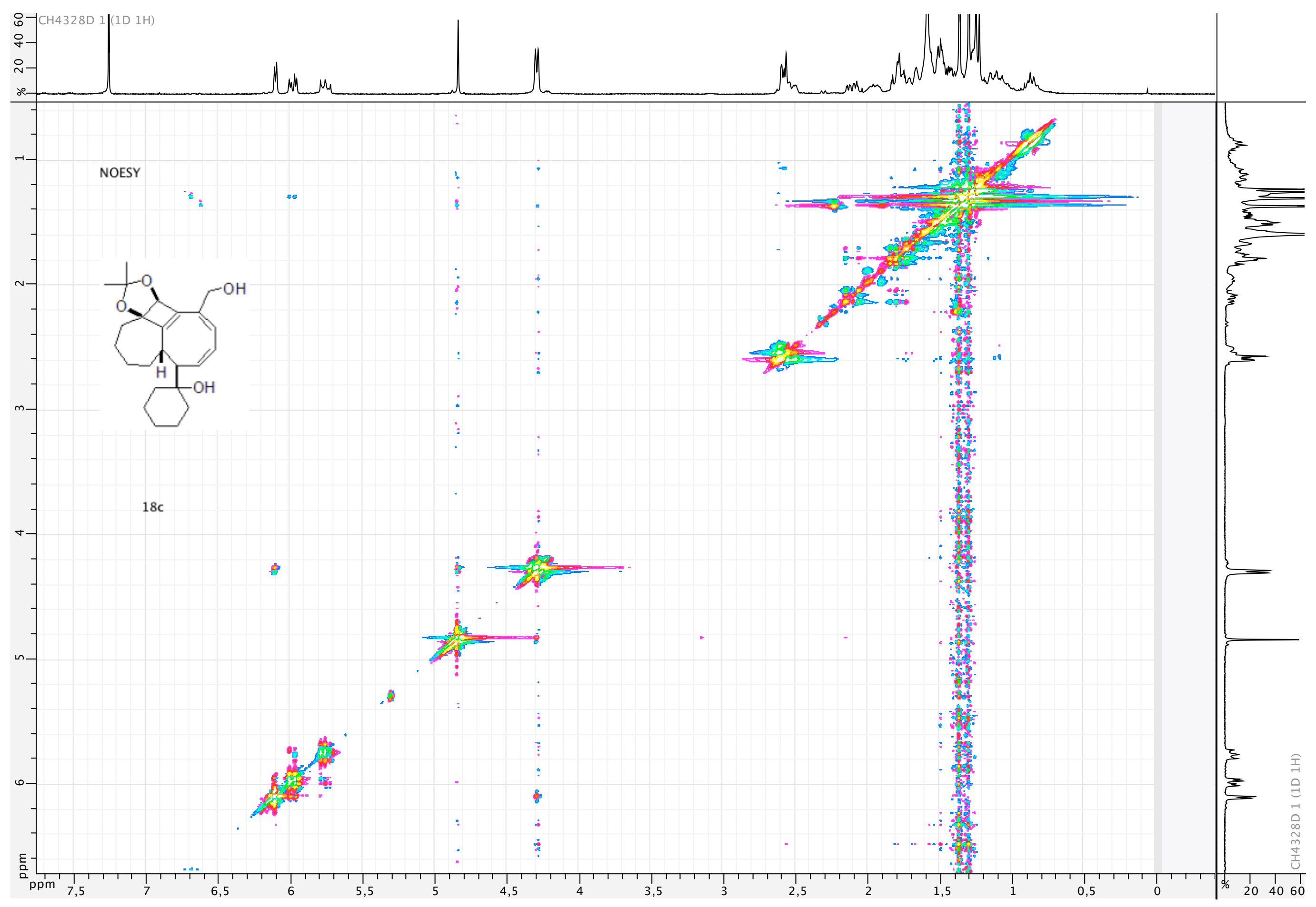




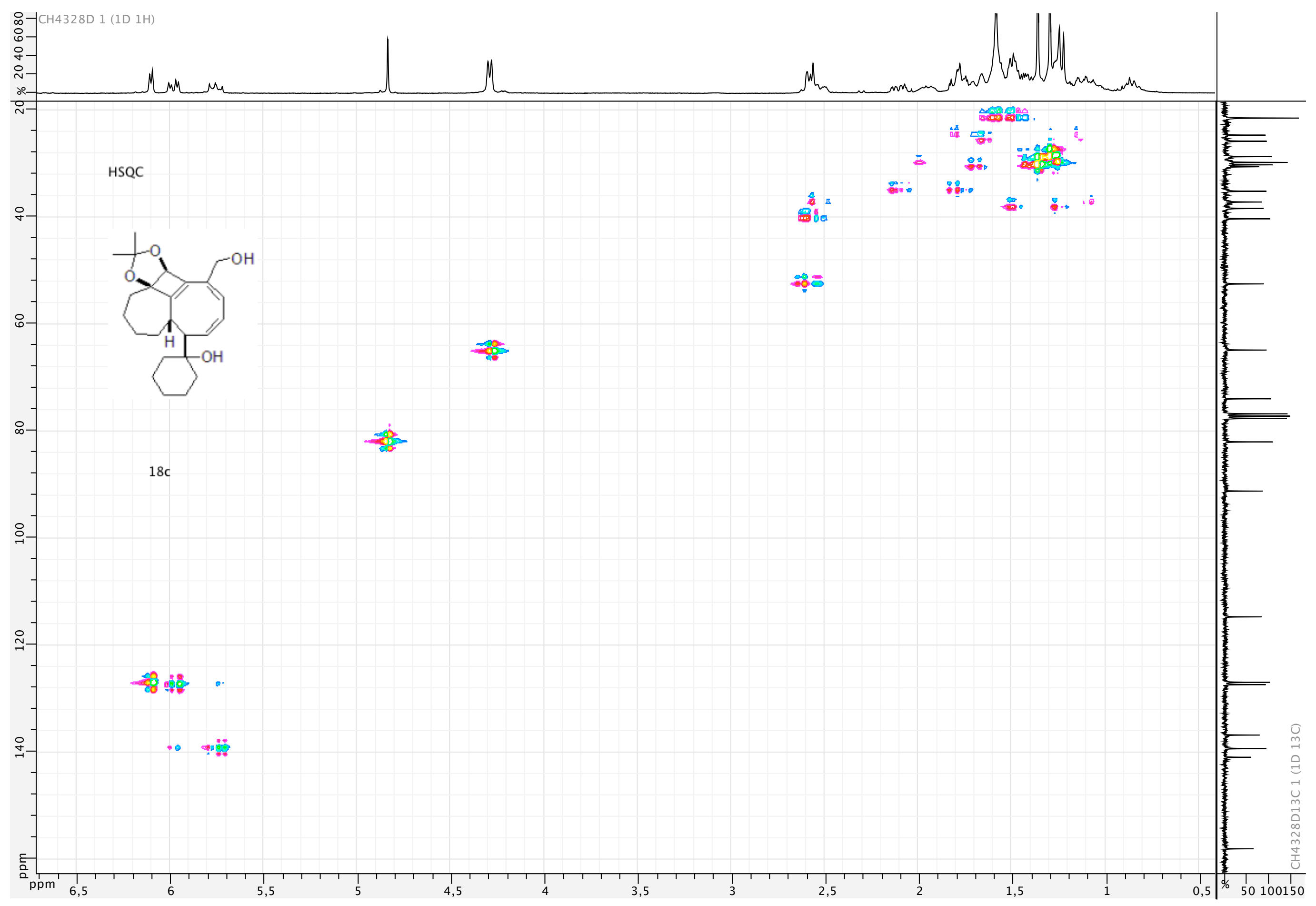




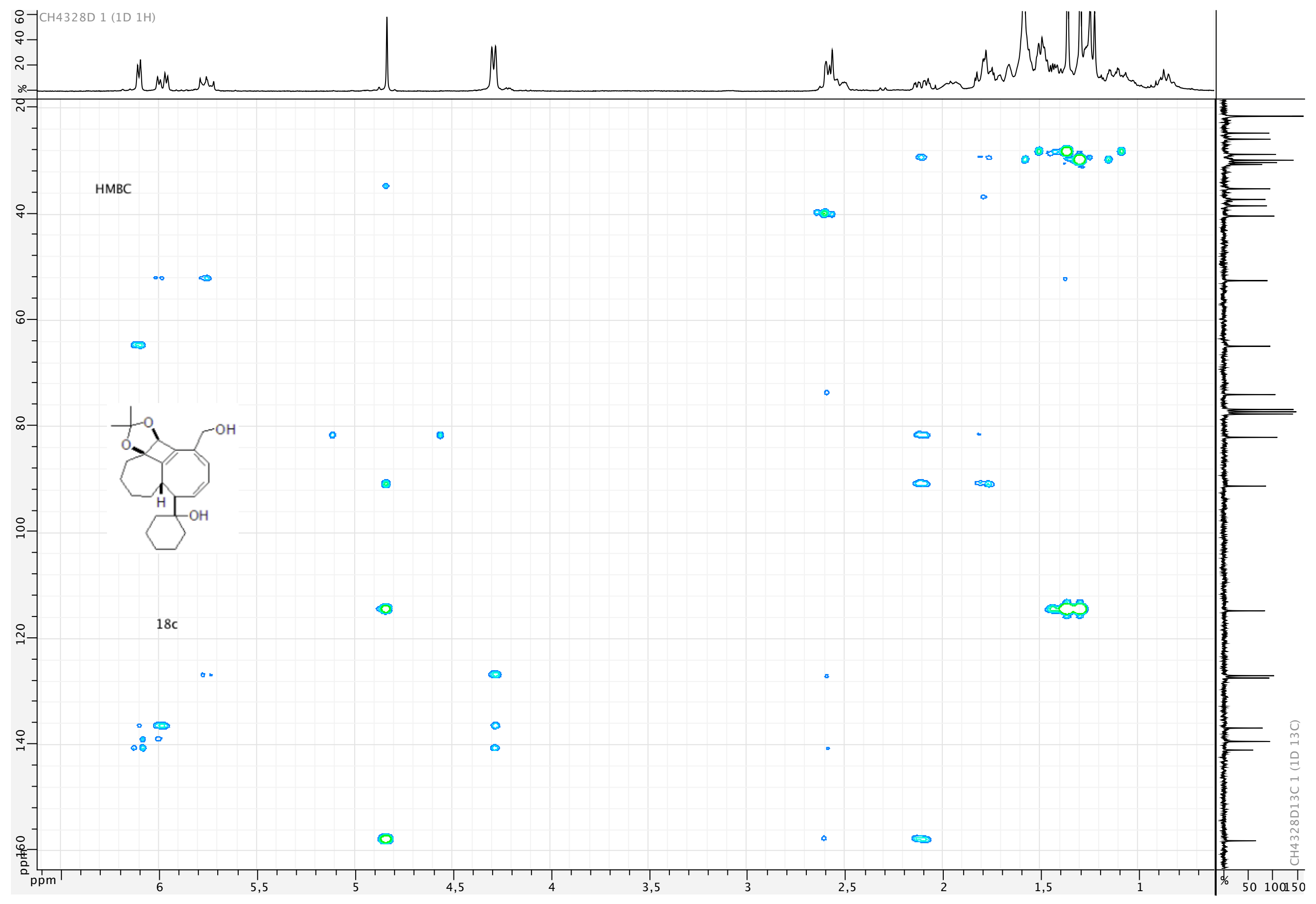




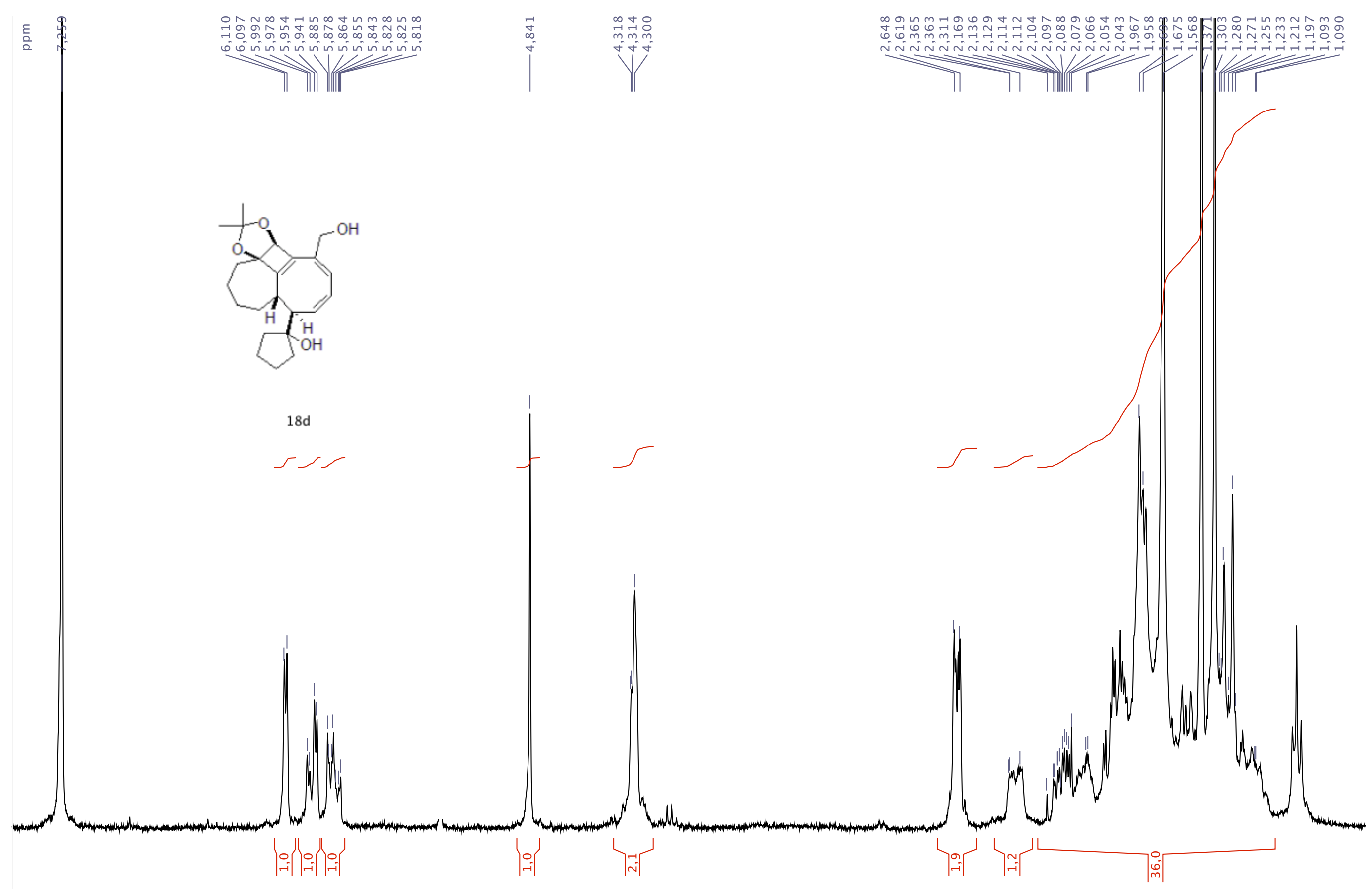




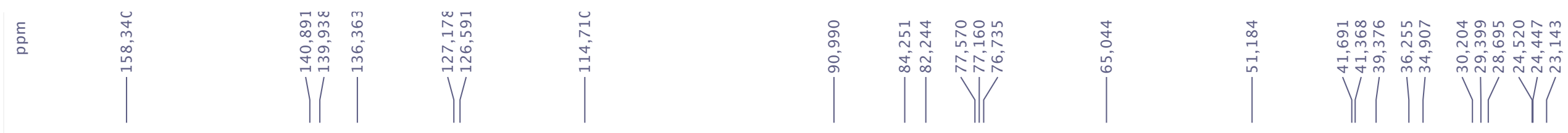
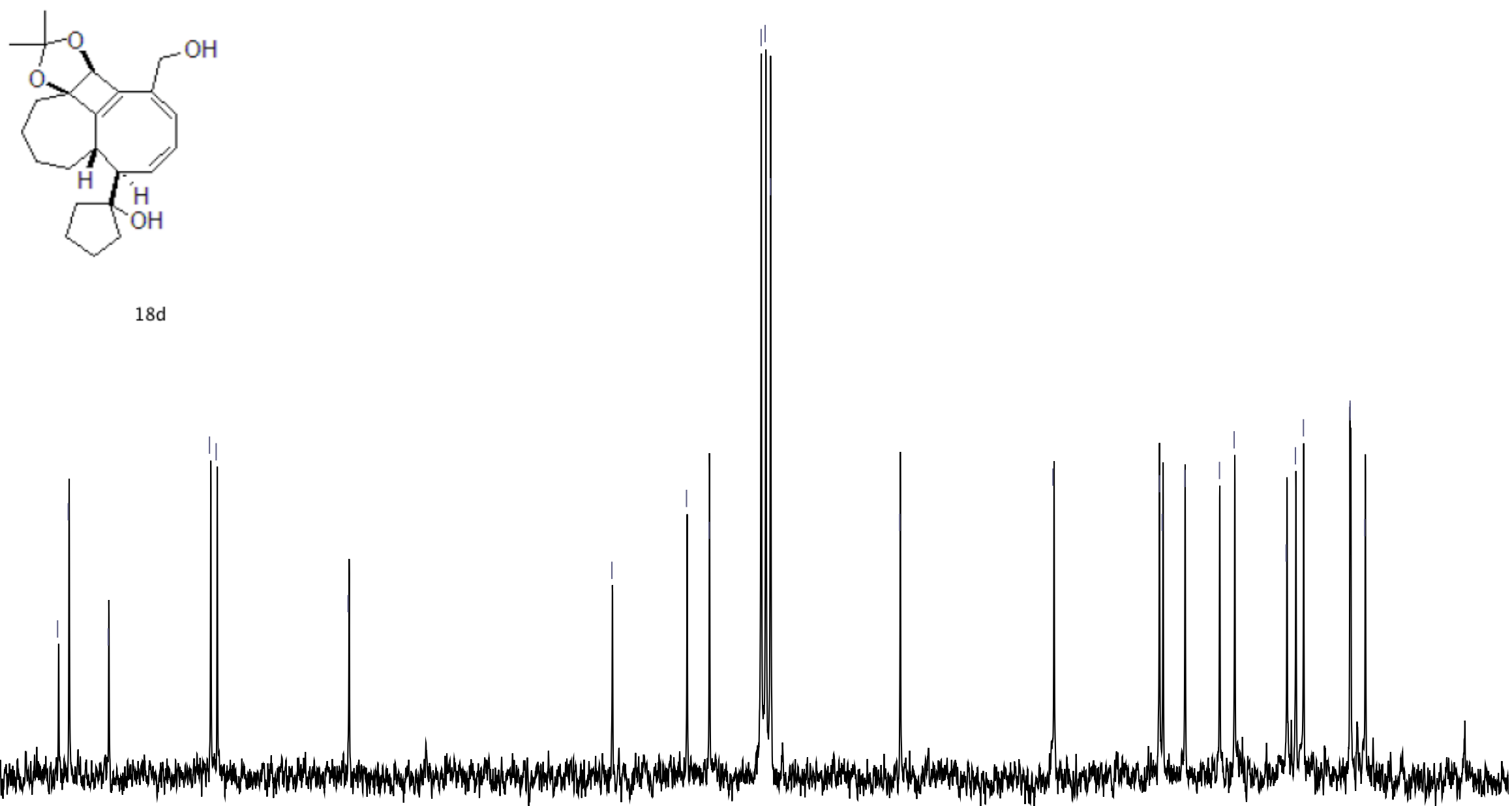


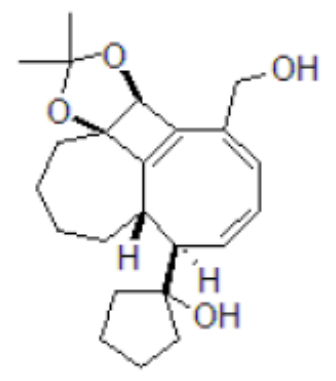

$18 d$

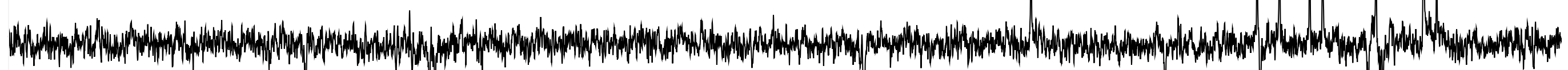

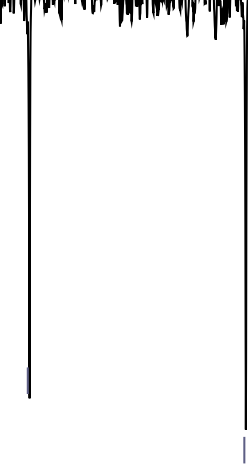




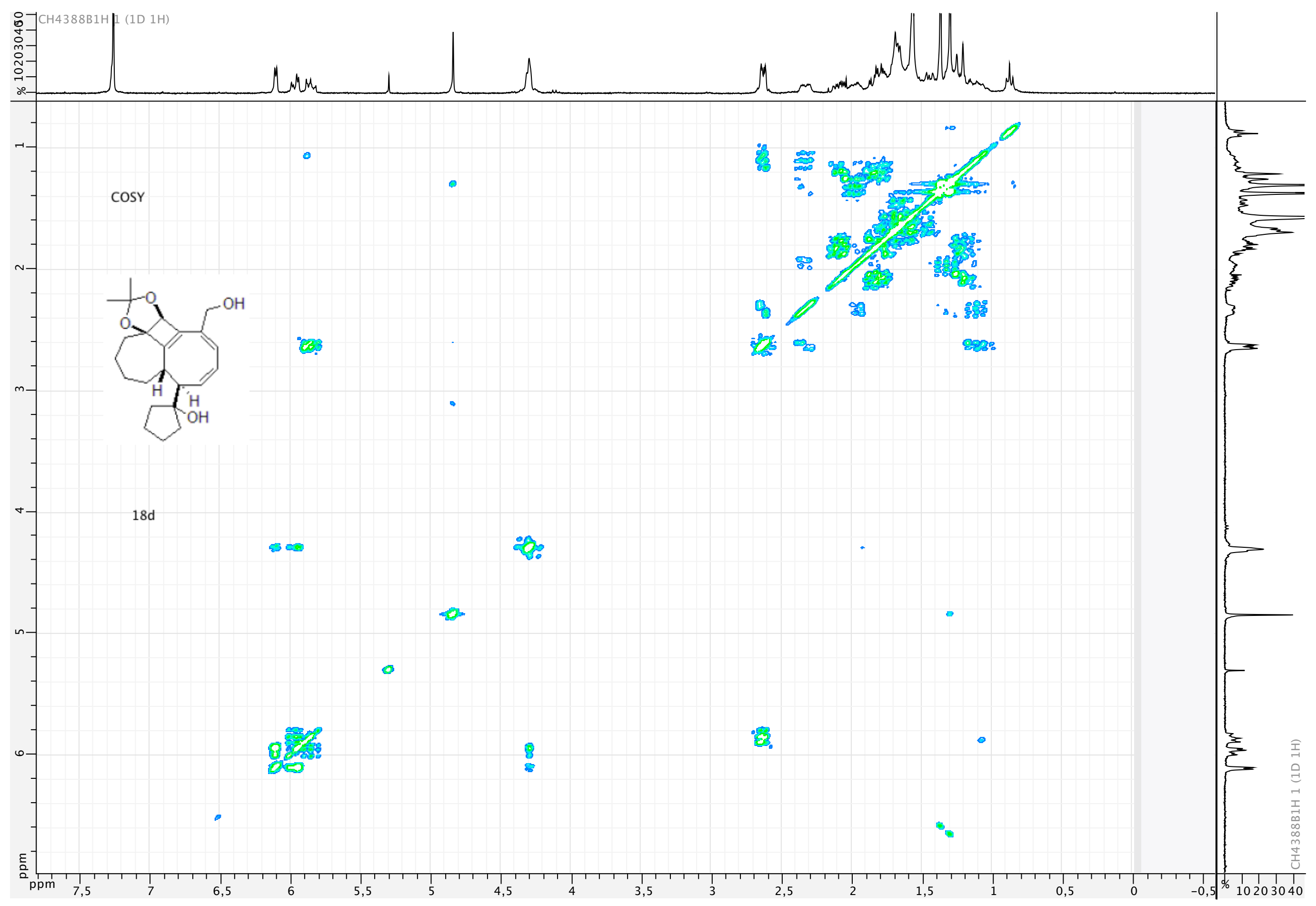




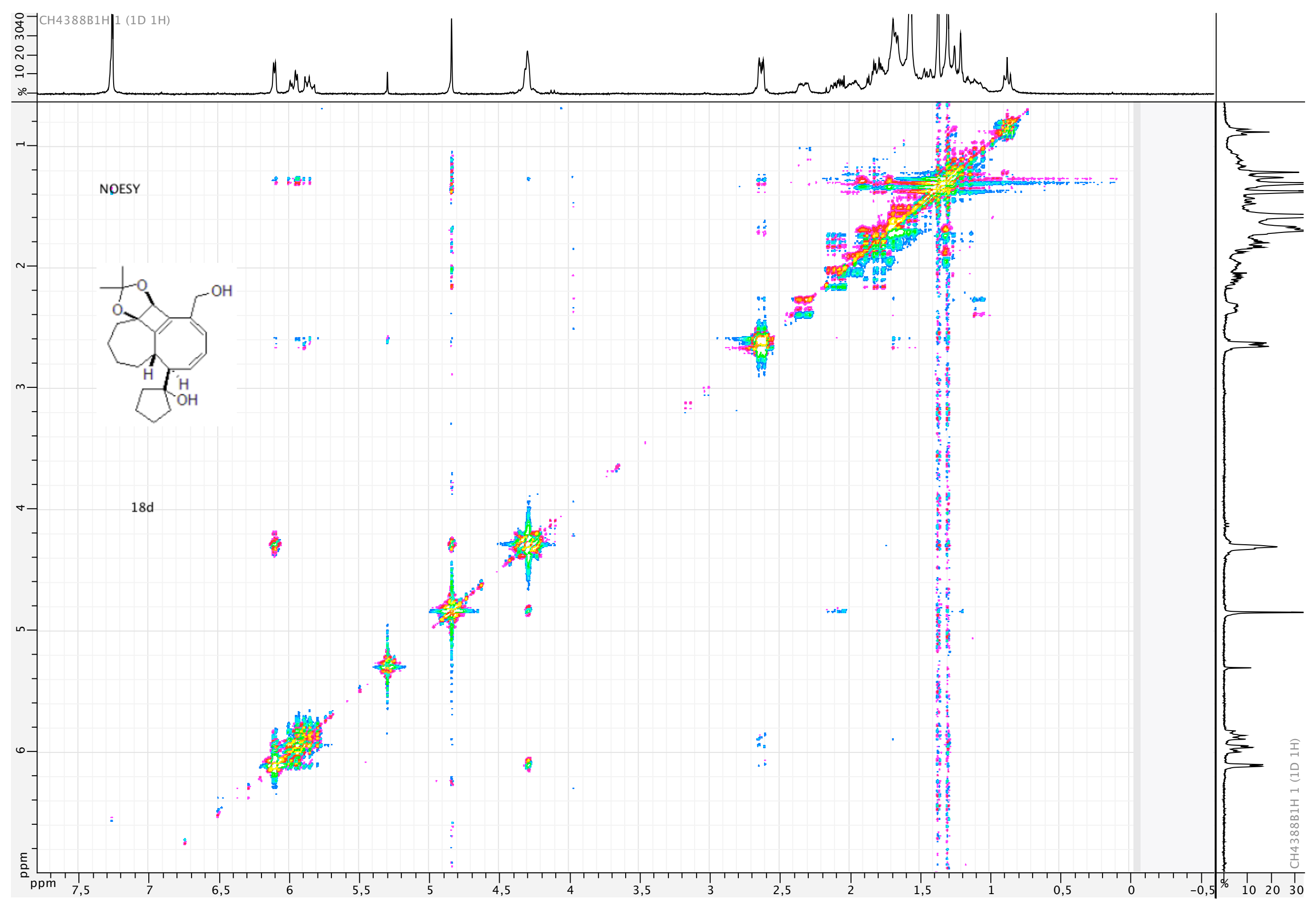




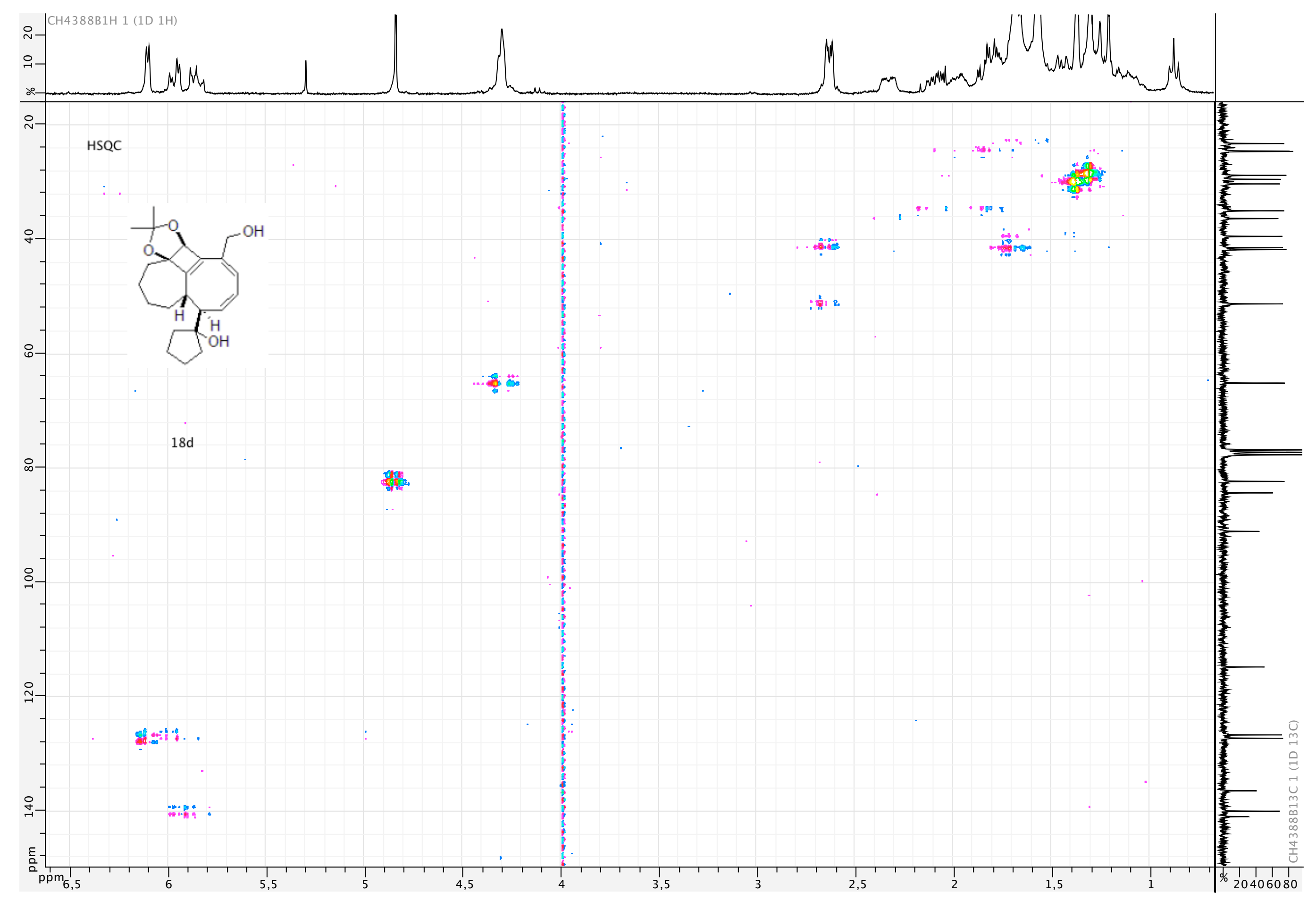




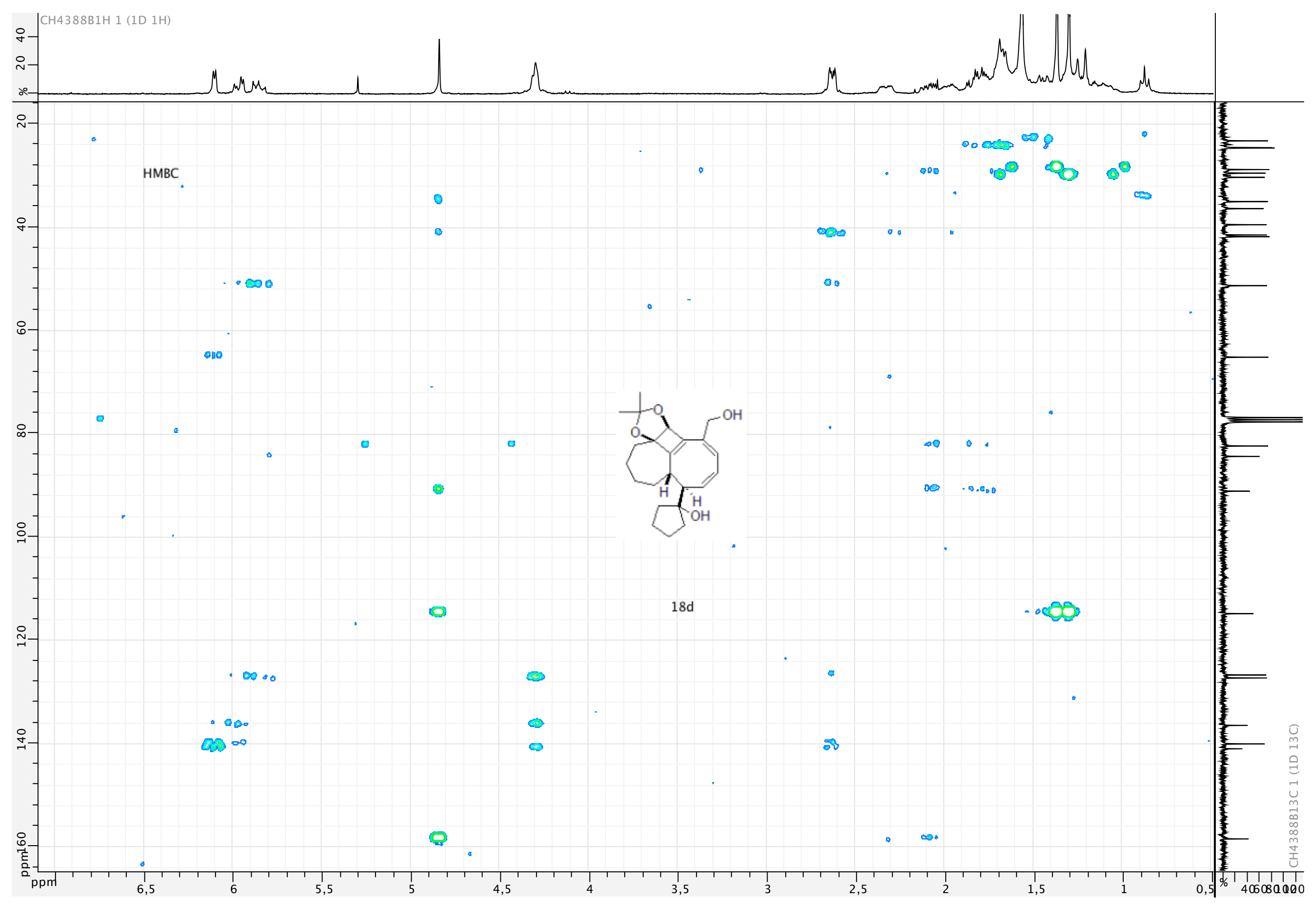




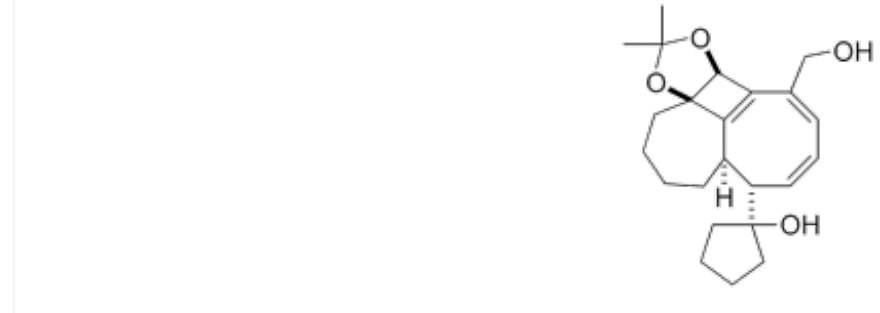

$18 \mathrm{~d}^{\prime}$
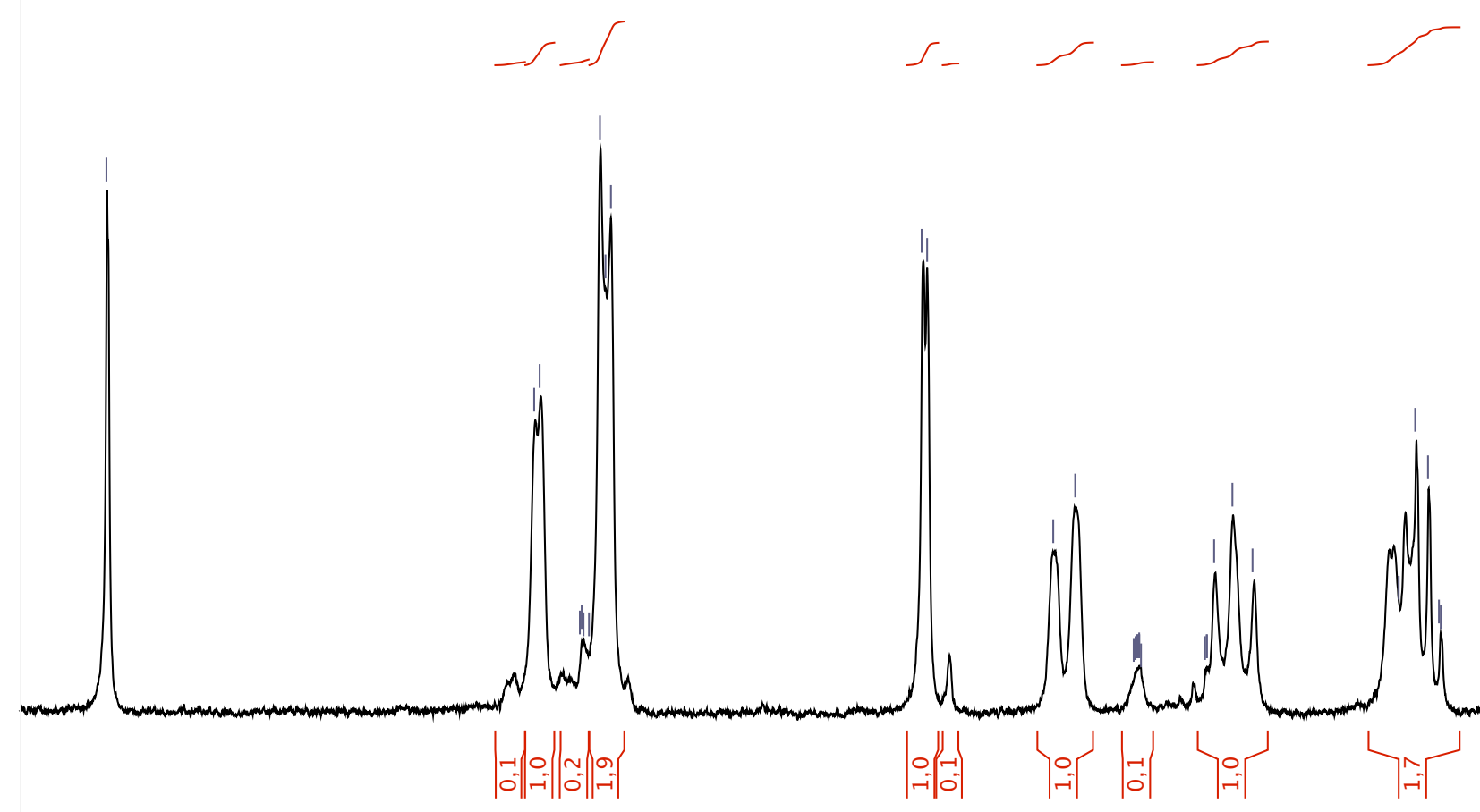
1

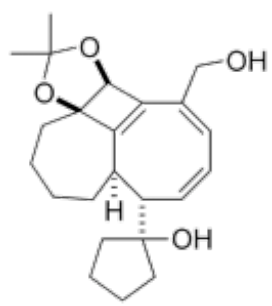

$18 d^{\prime}$

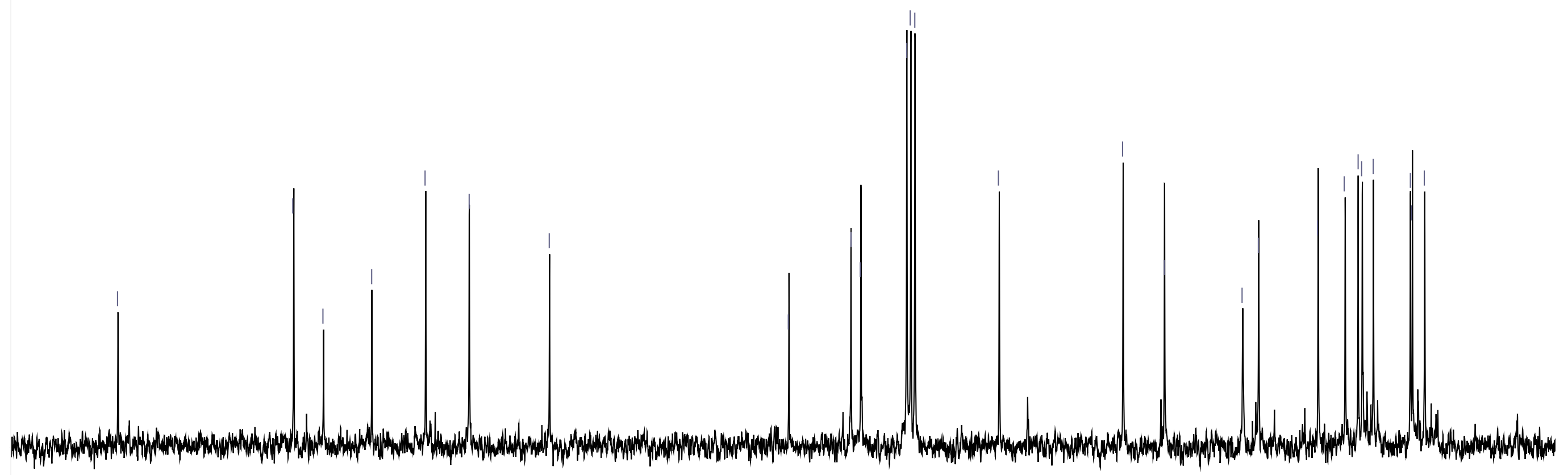




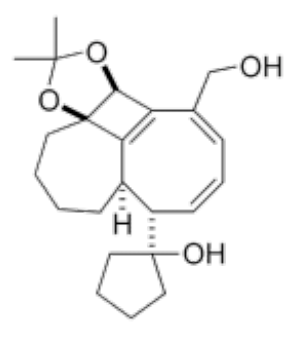




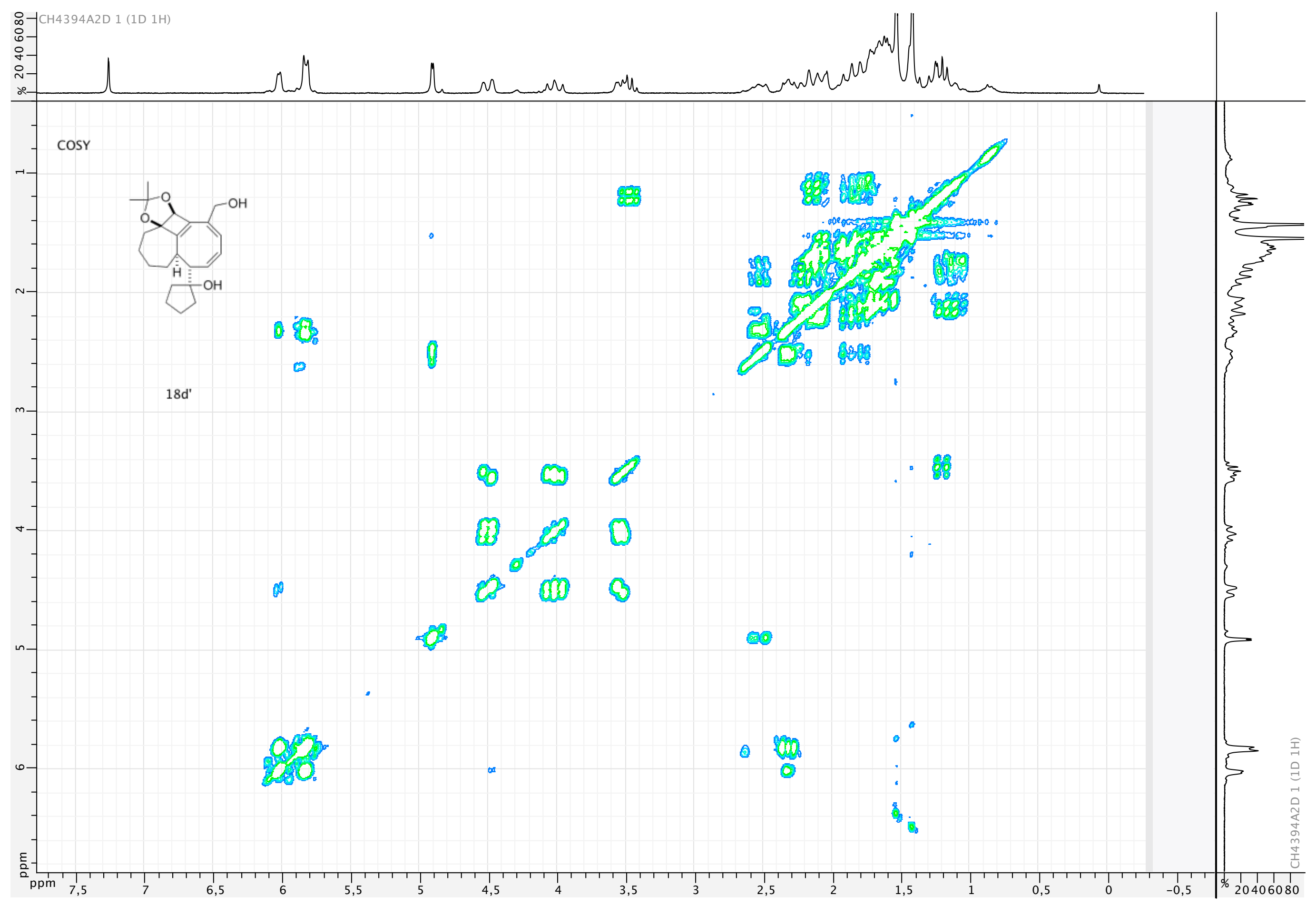




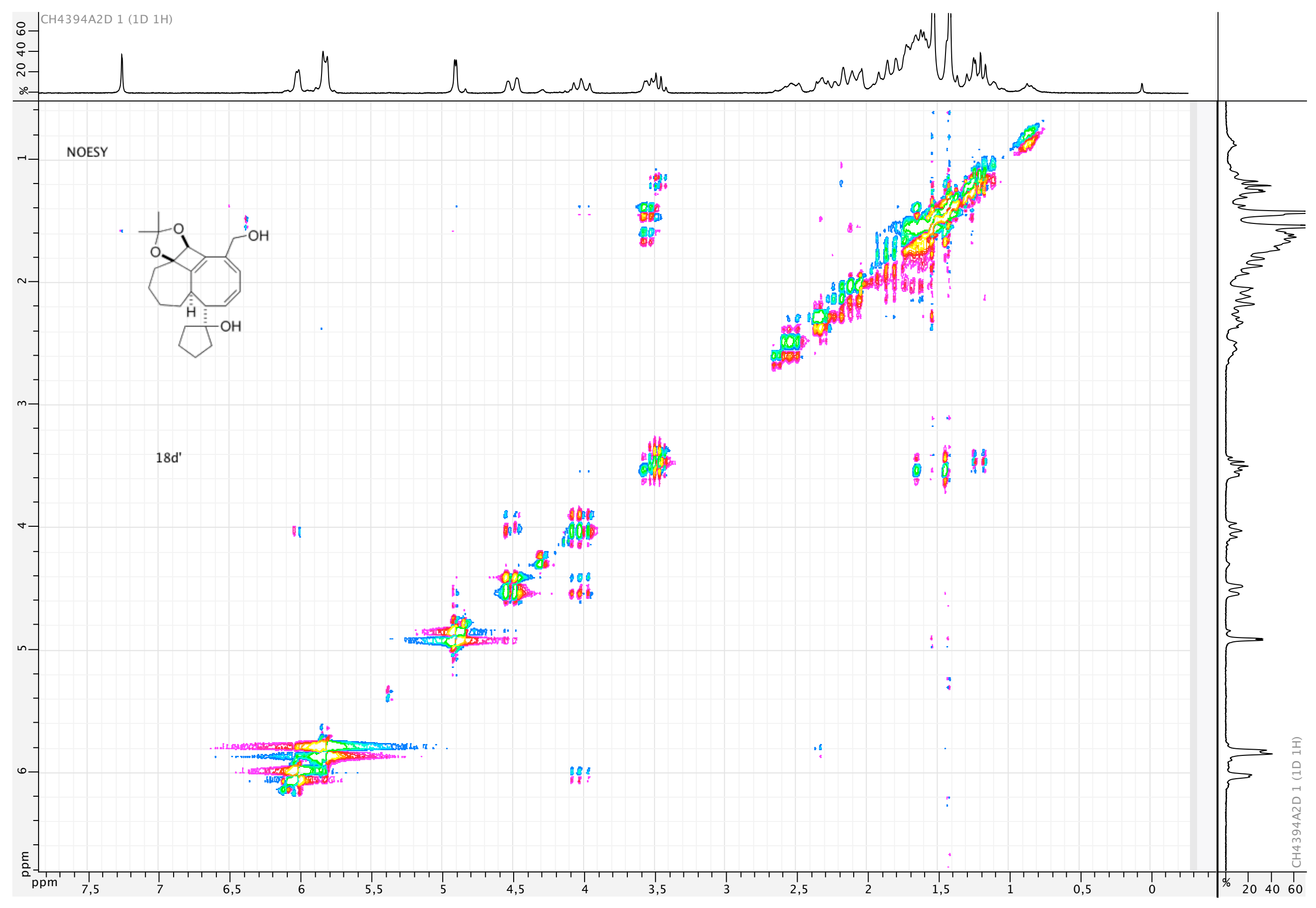




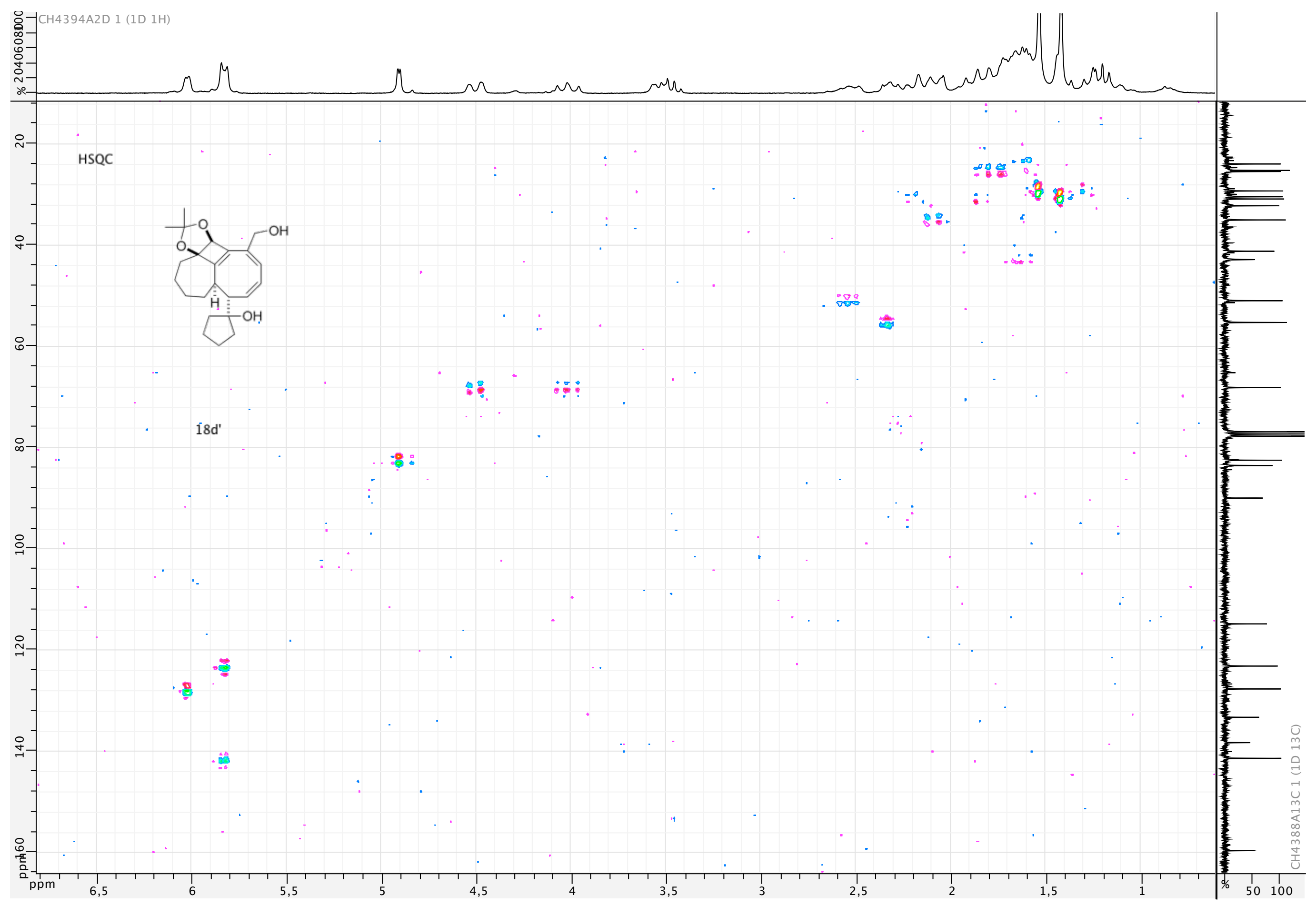




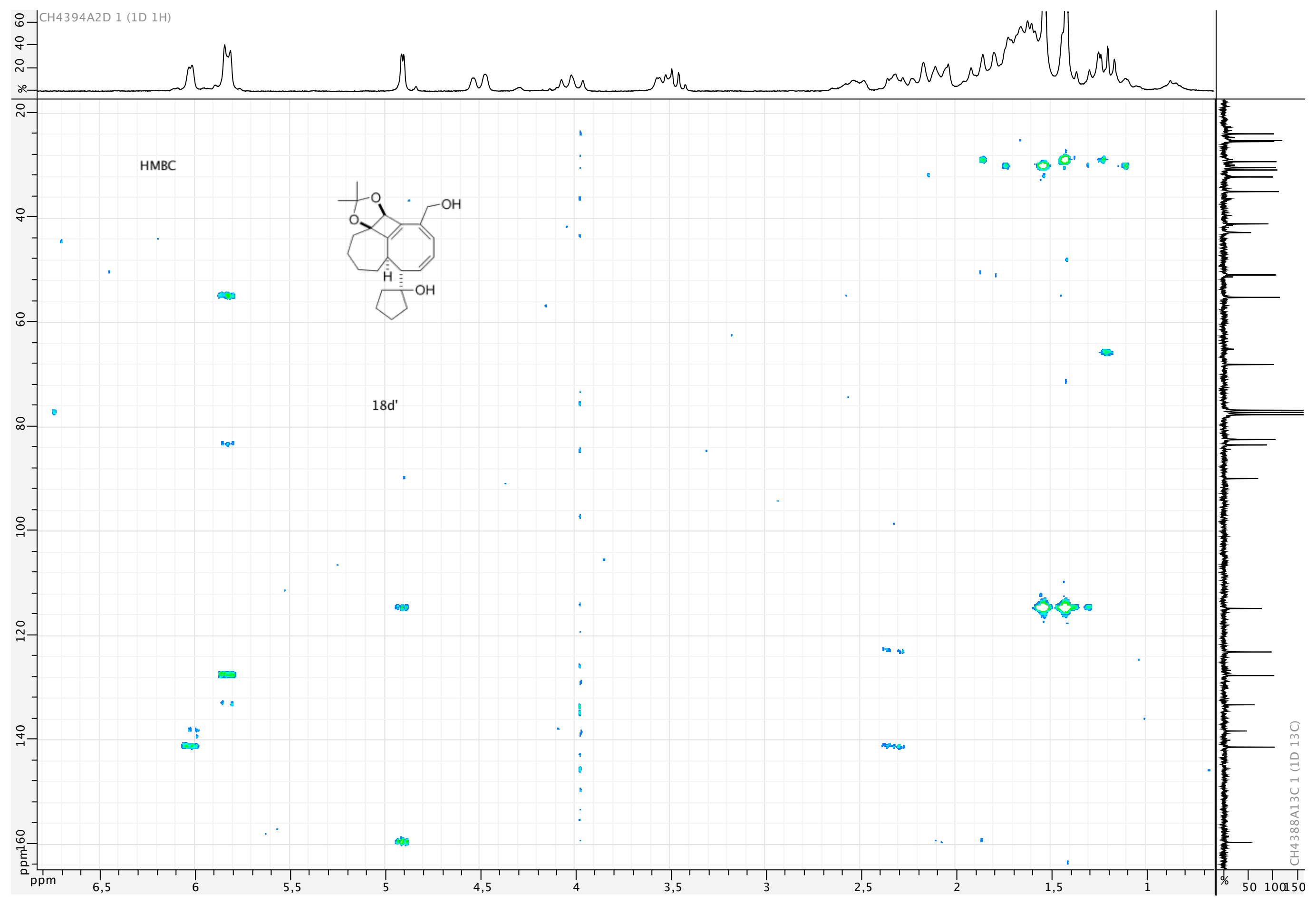



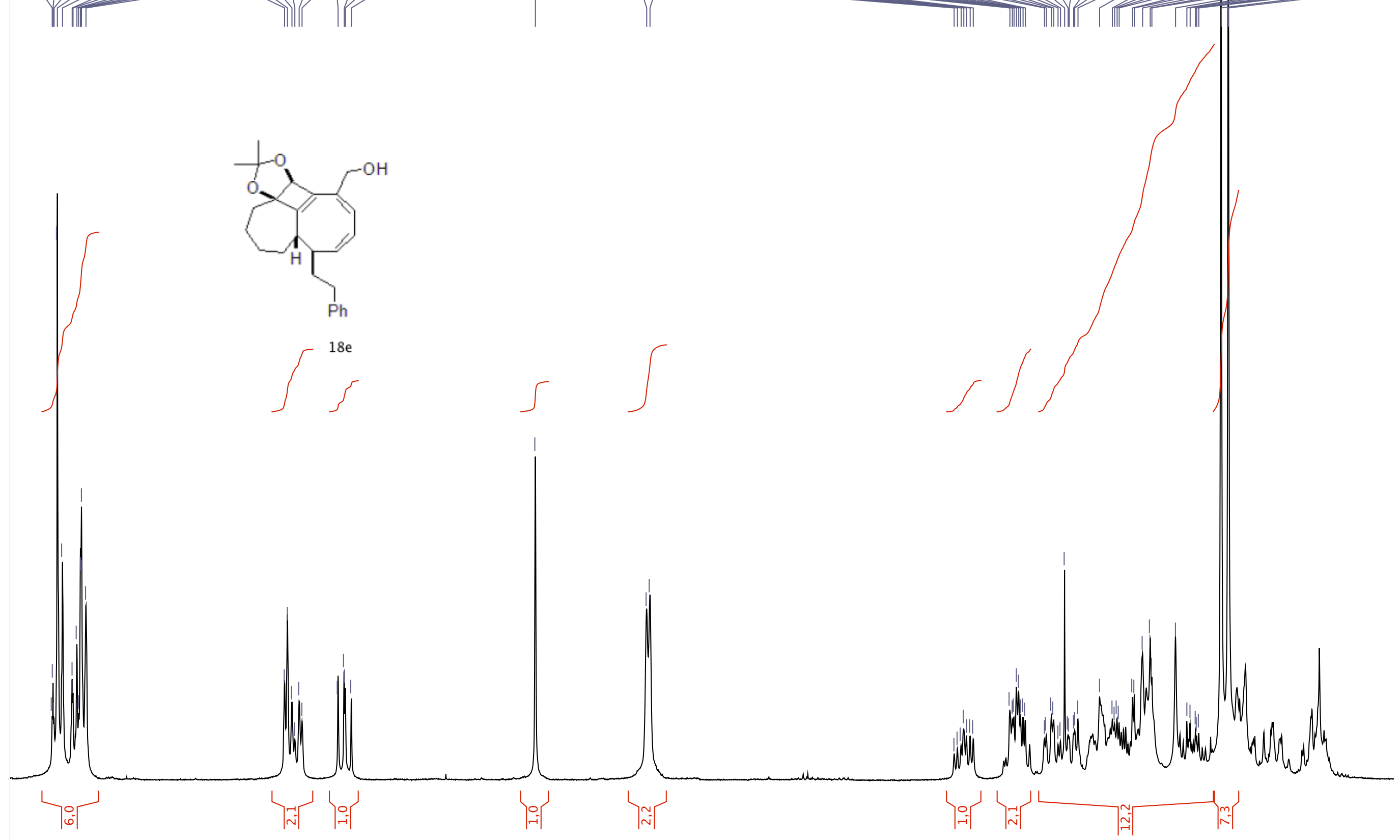

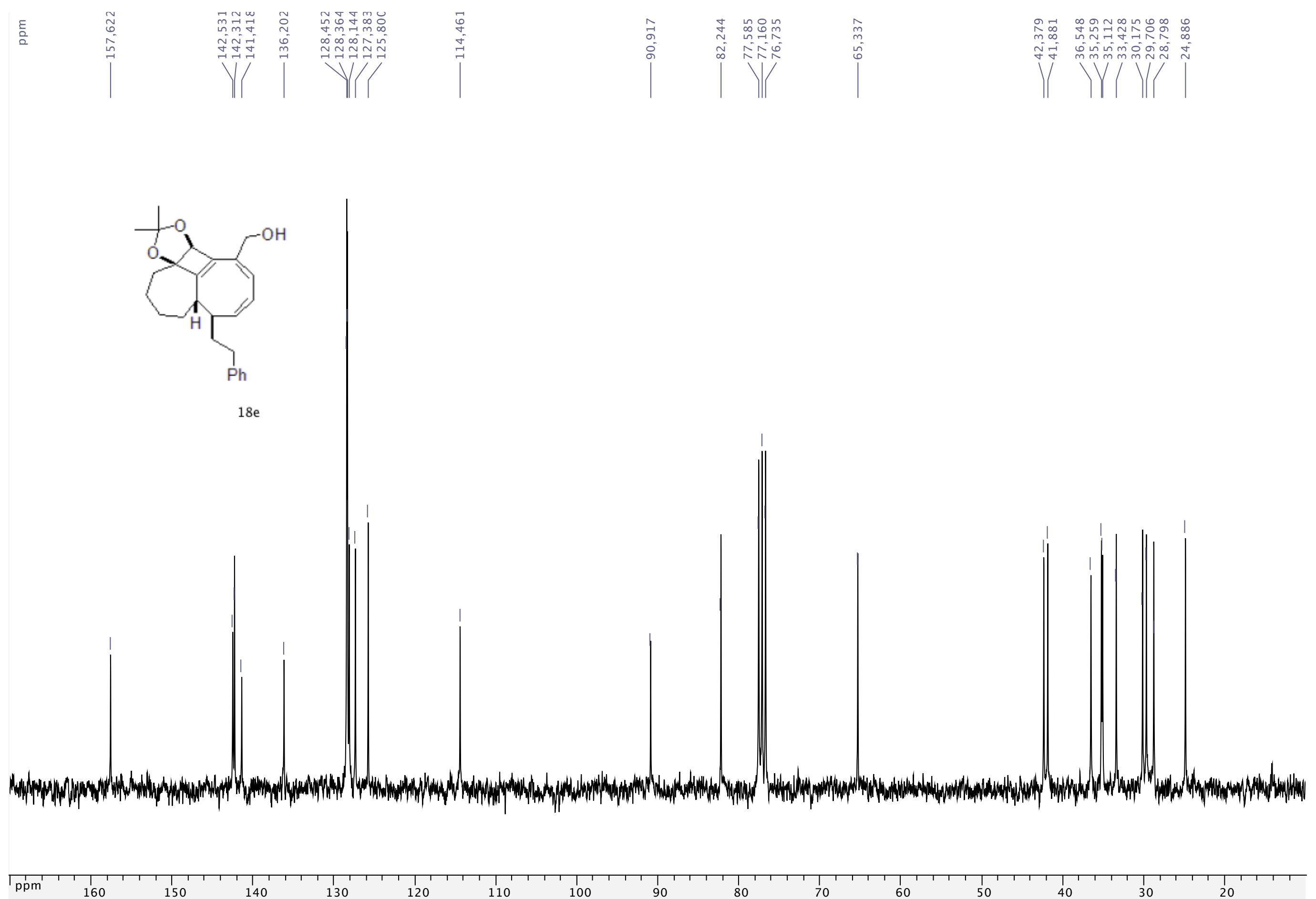

130

120

110

100

90 


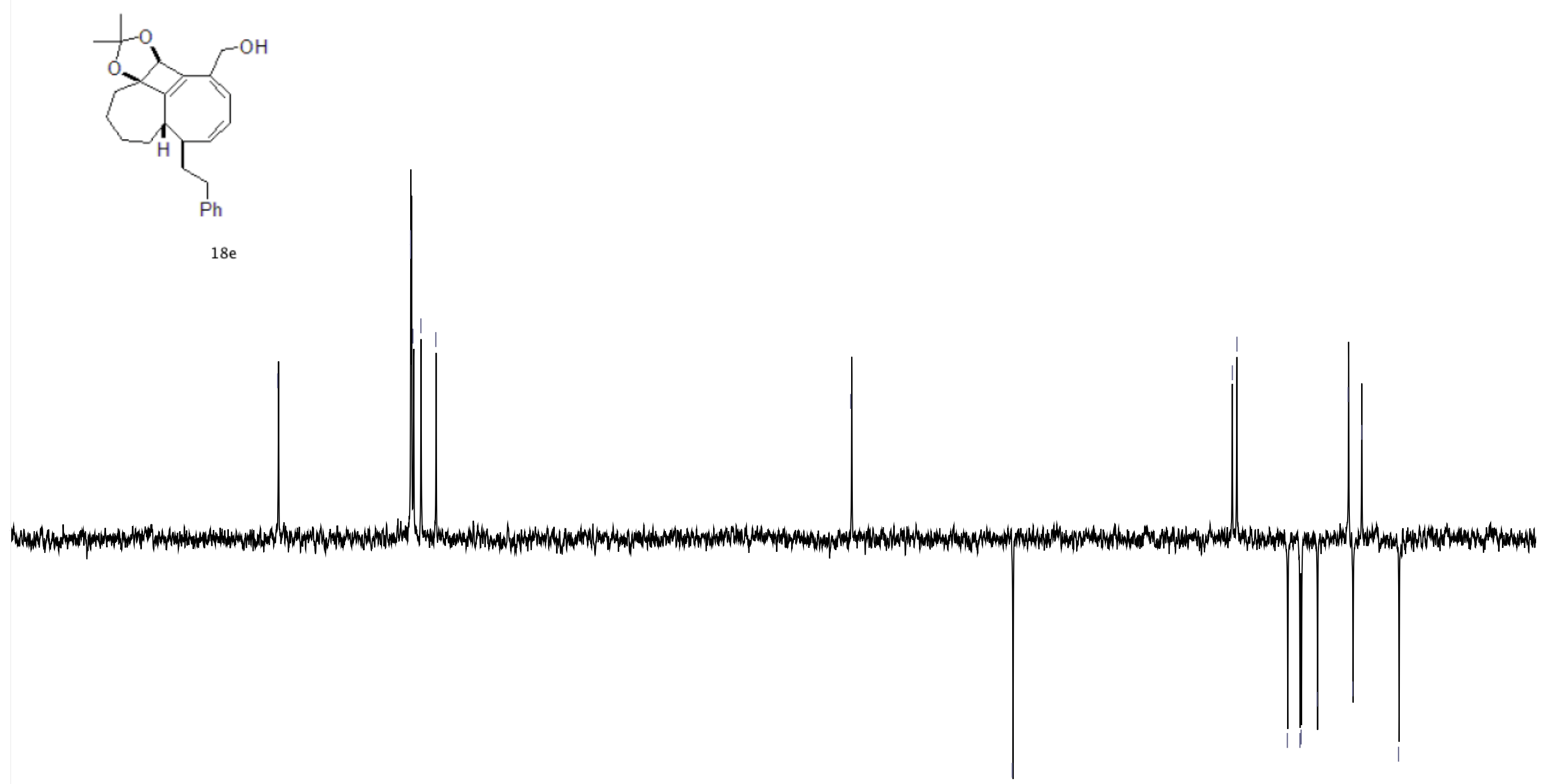




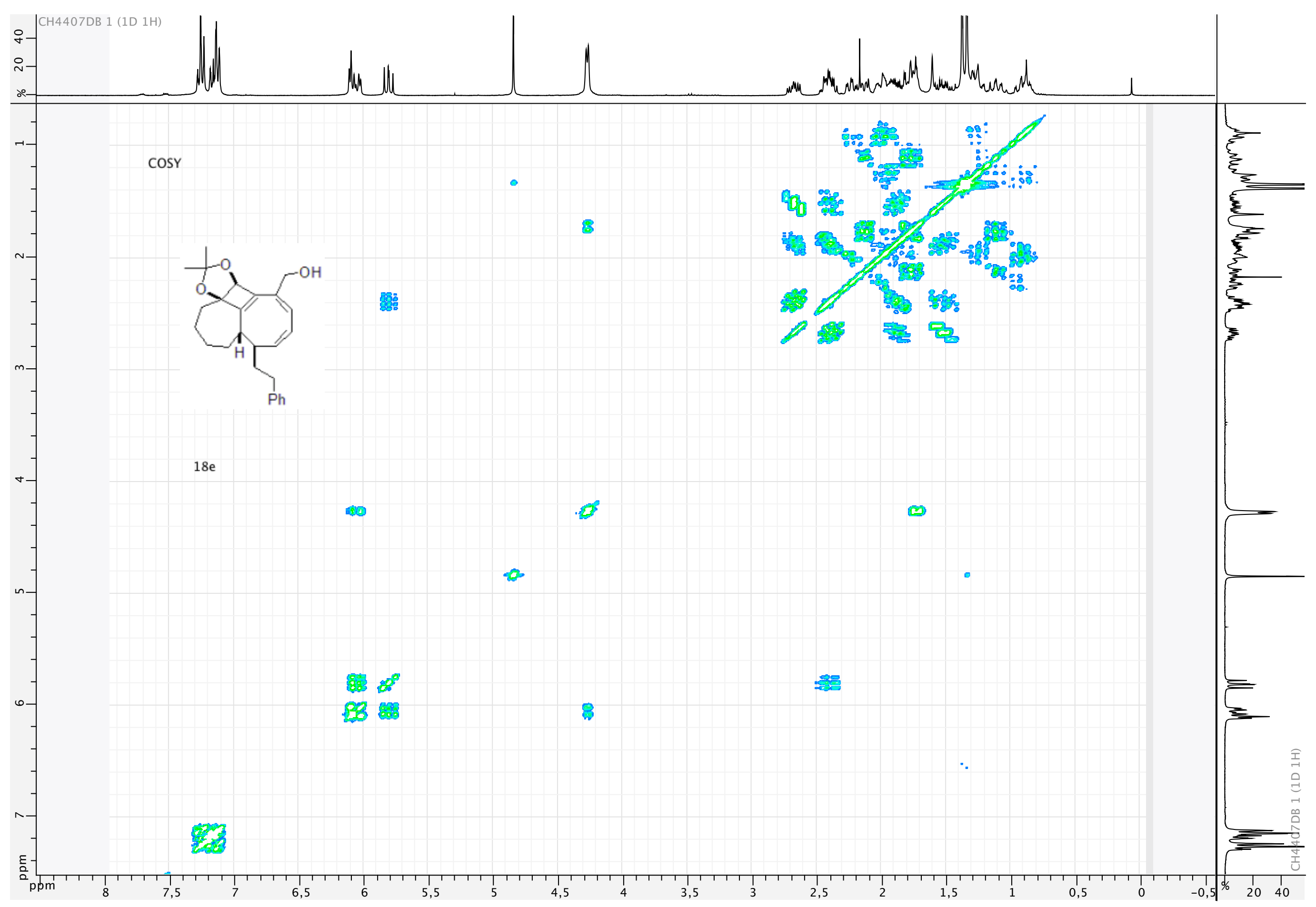




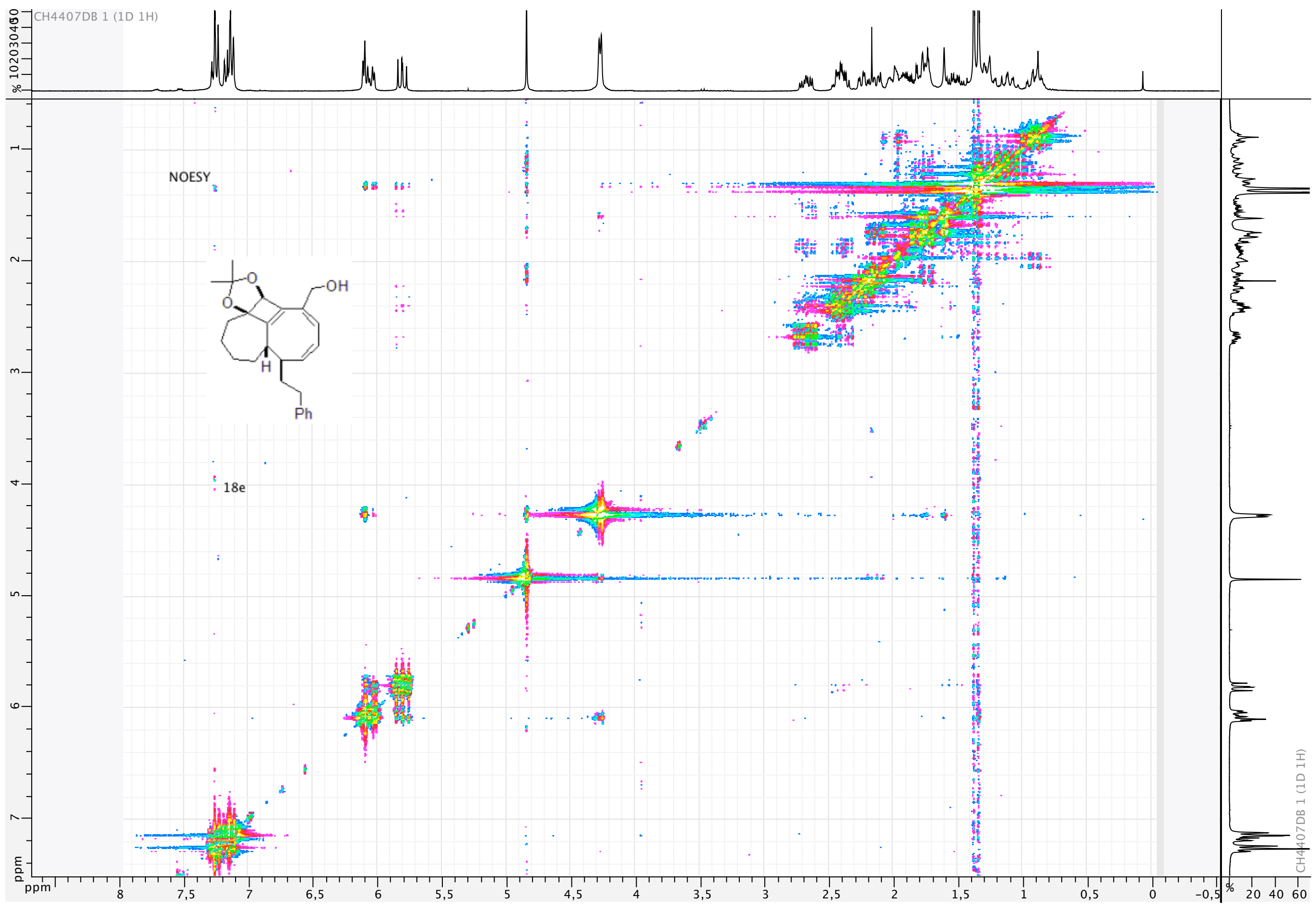




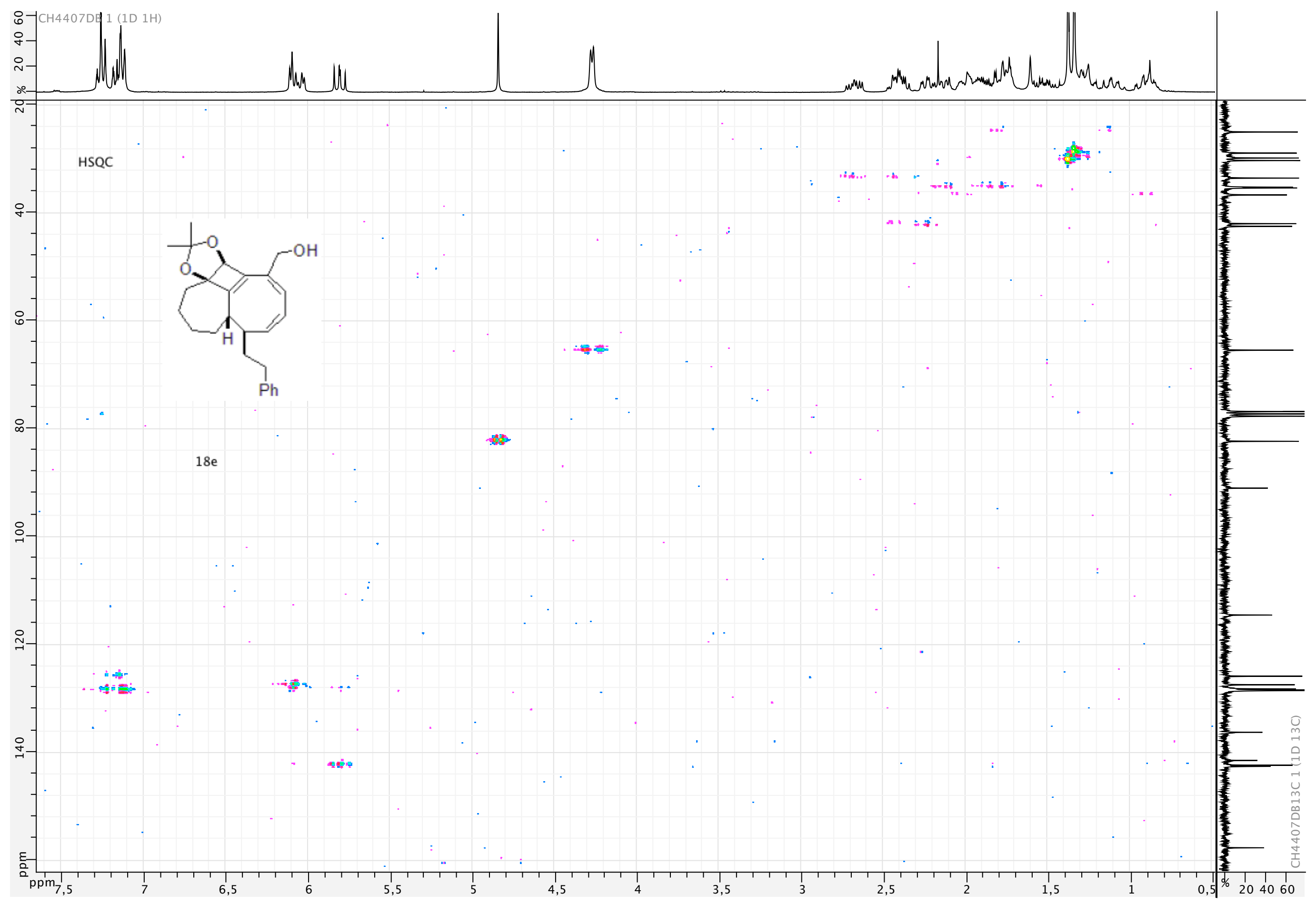




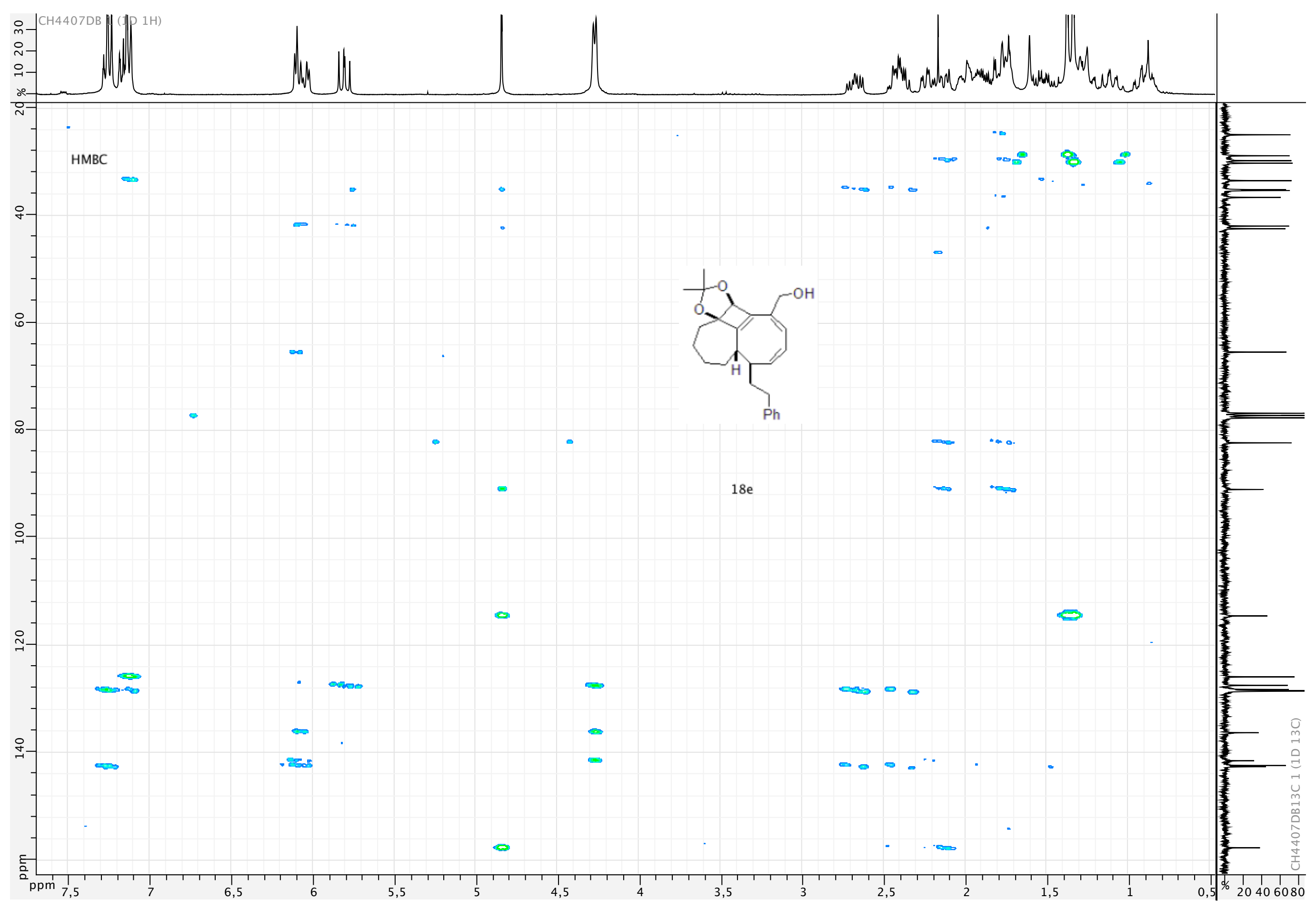




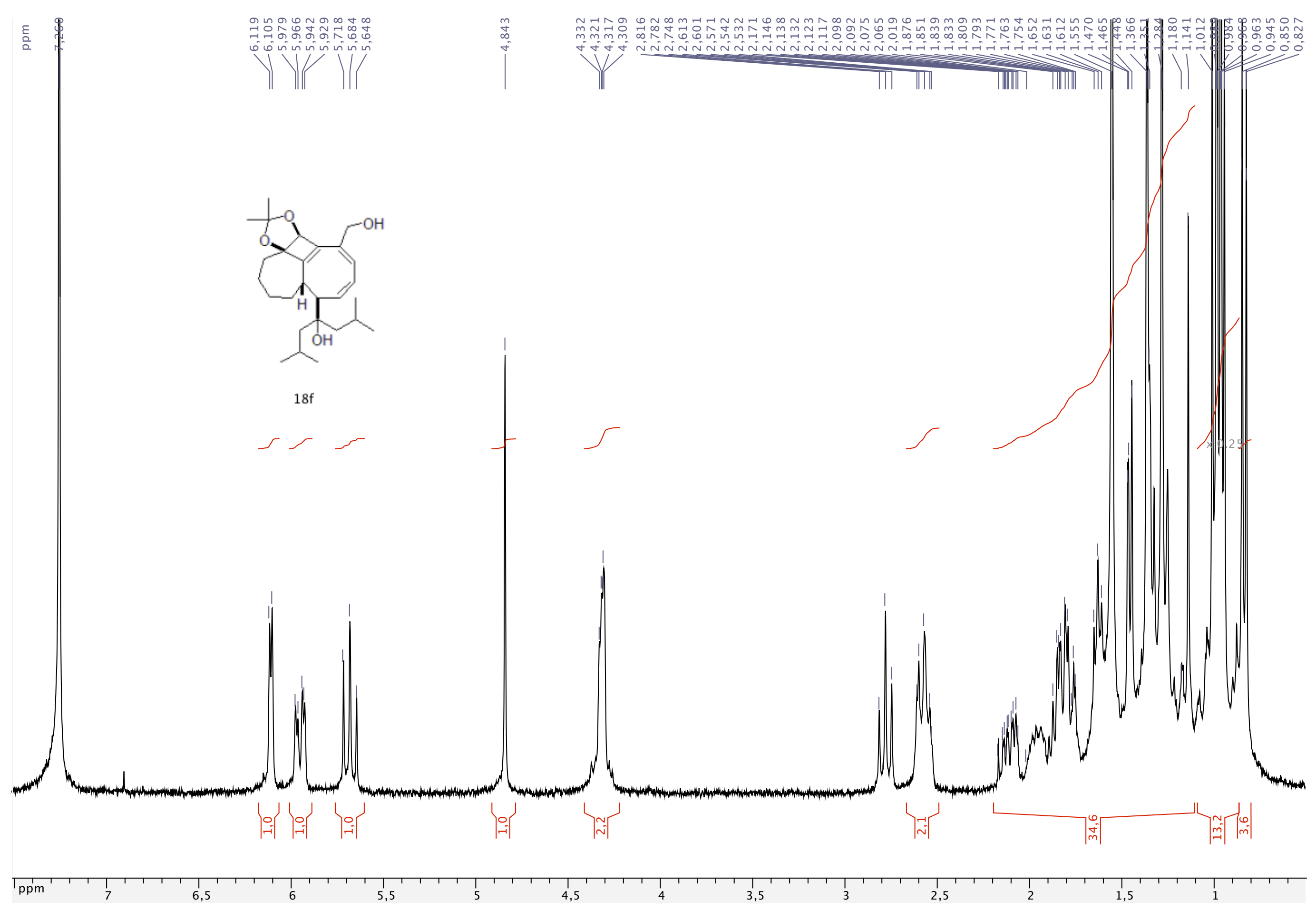


ह

$\left.\right|^{1}$

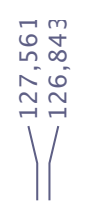

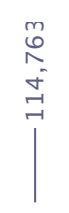

$\substack{0 \\ 0 \\ 0 \\ 0 \\ 0}$
$\mid$
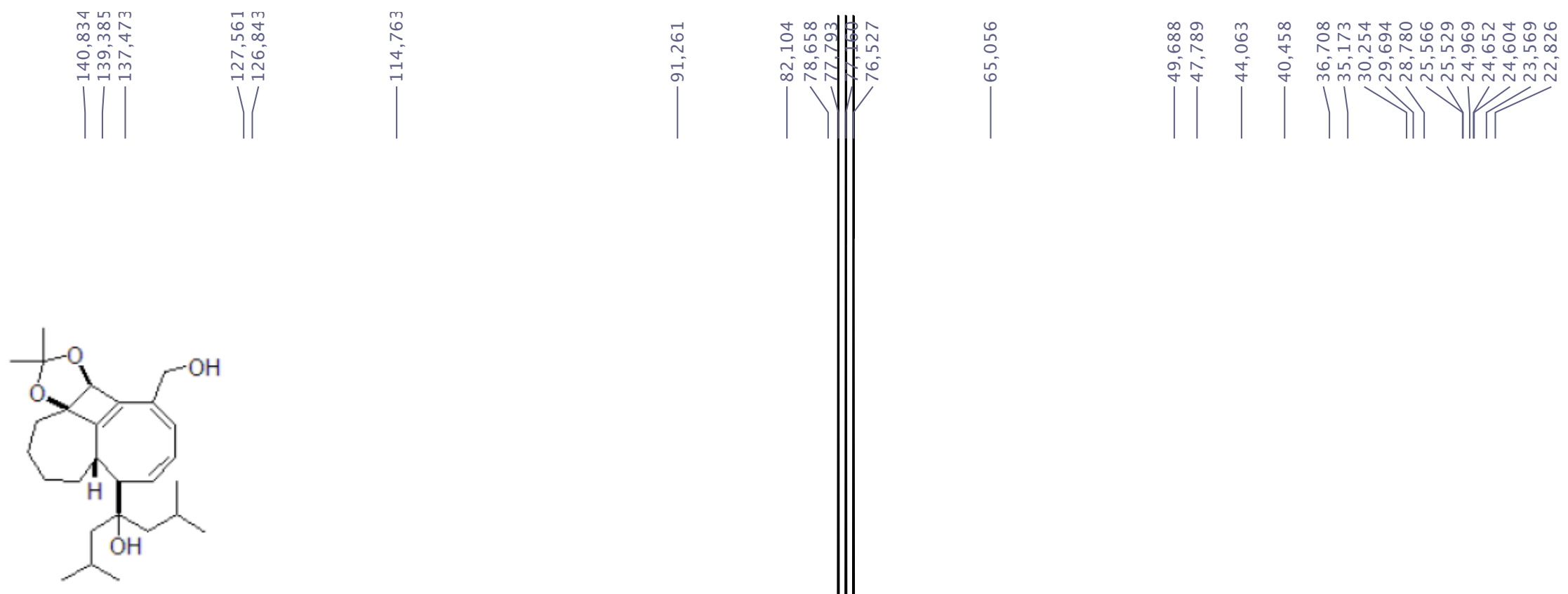

$18 \mathrm{f}$

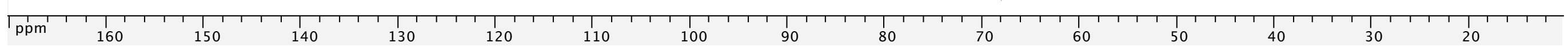




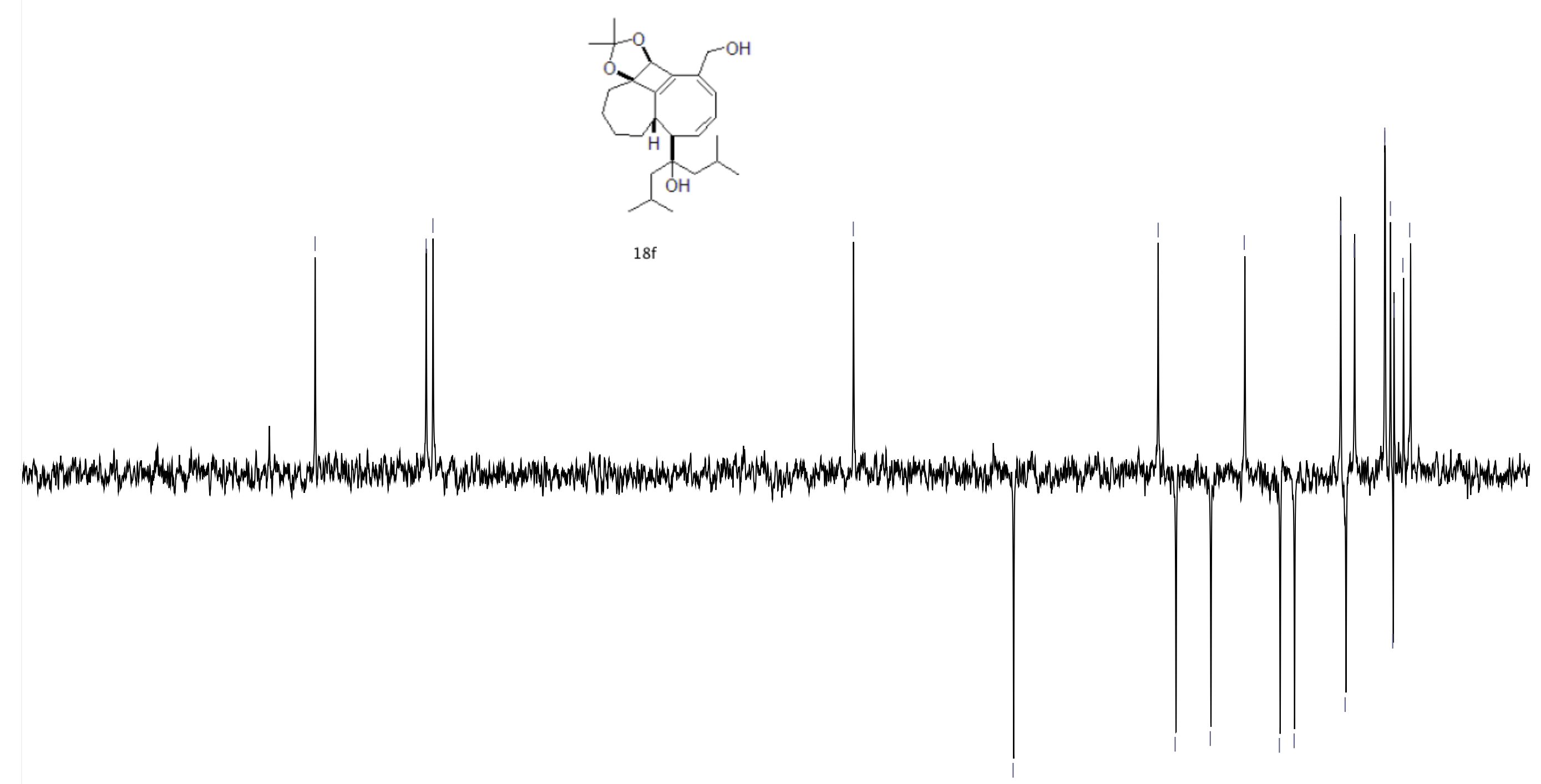




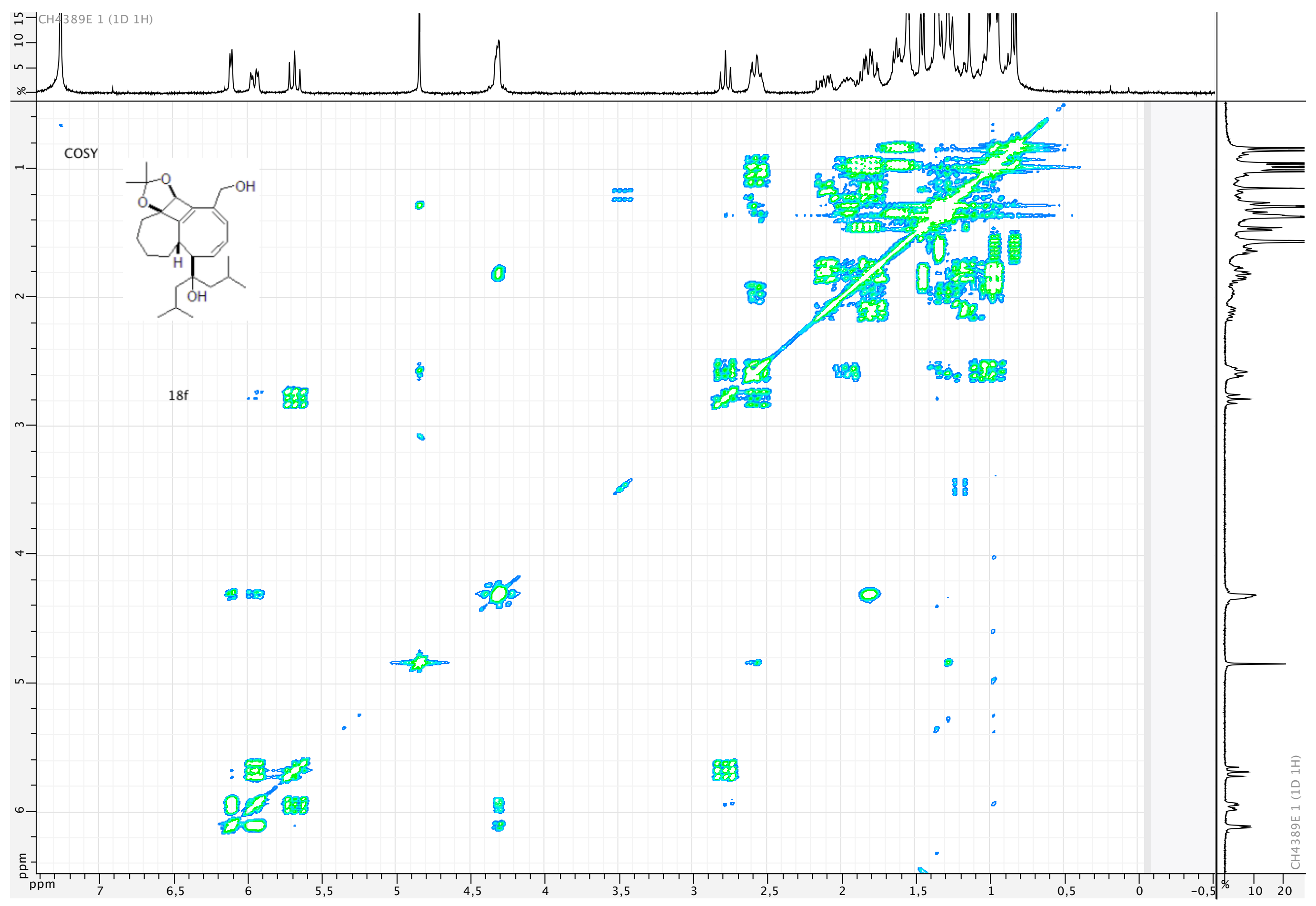




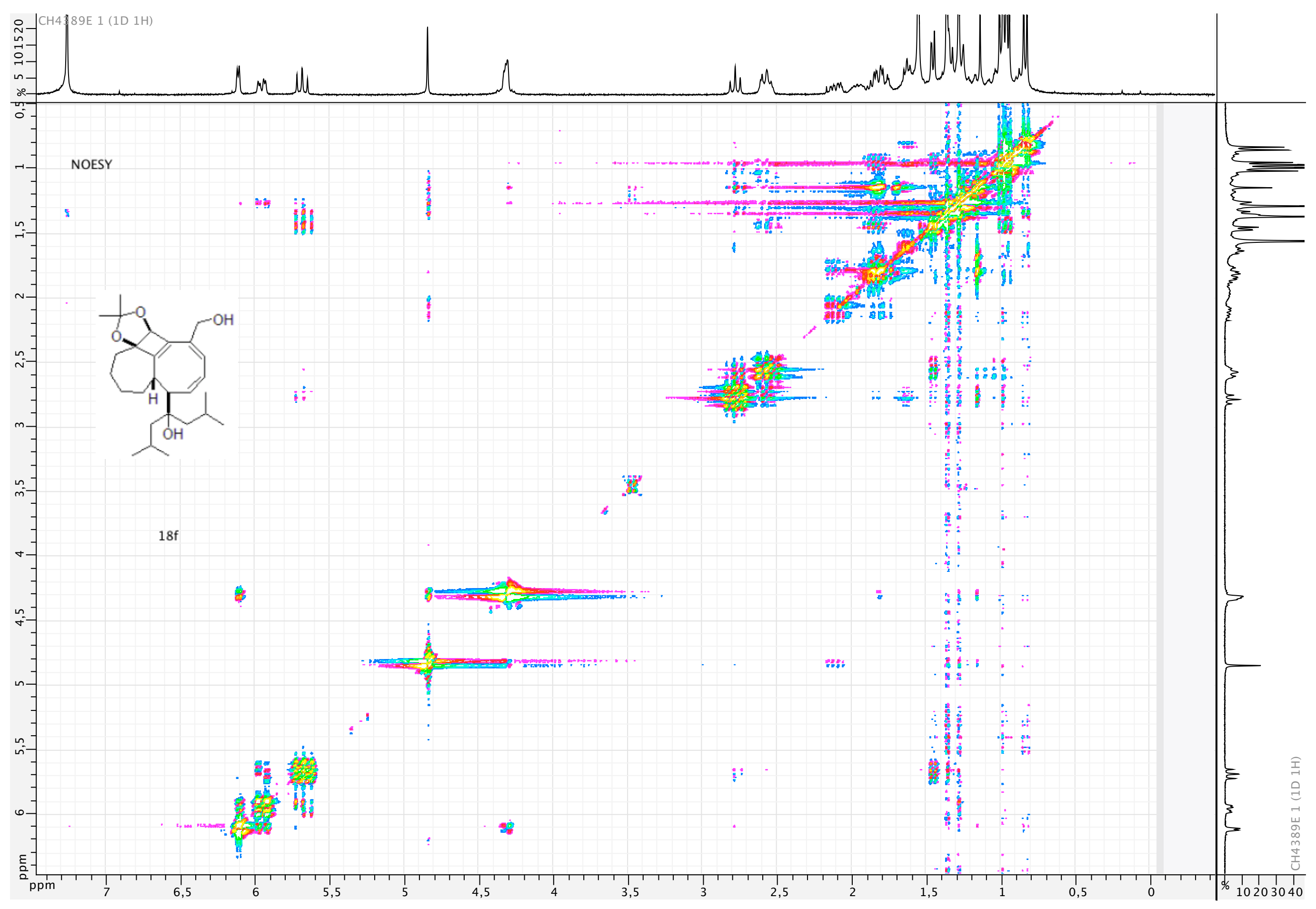




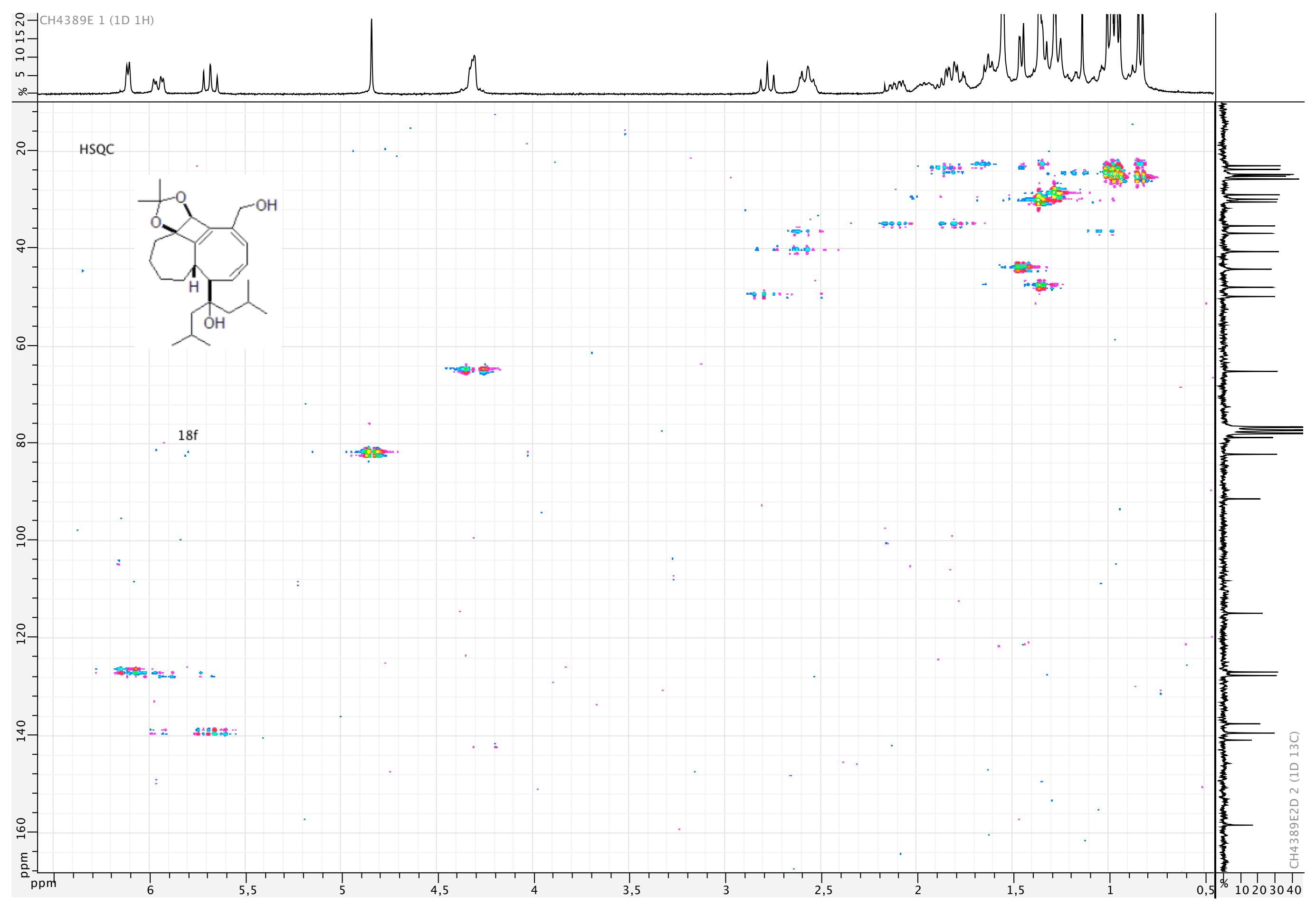




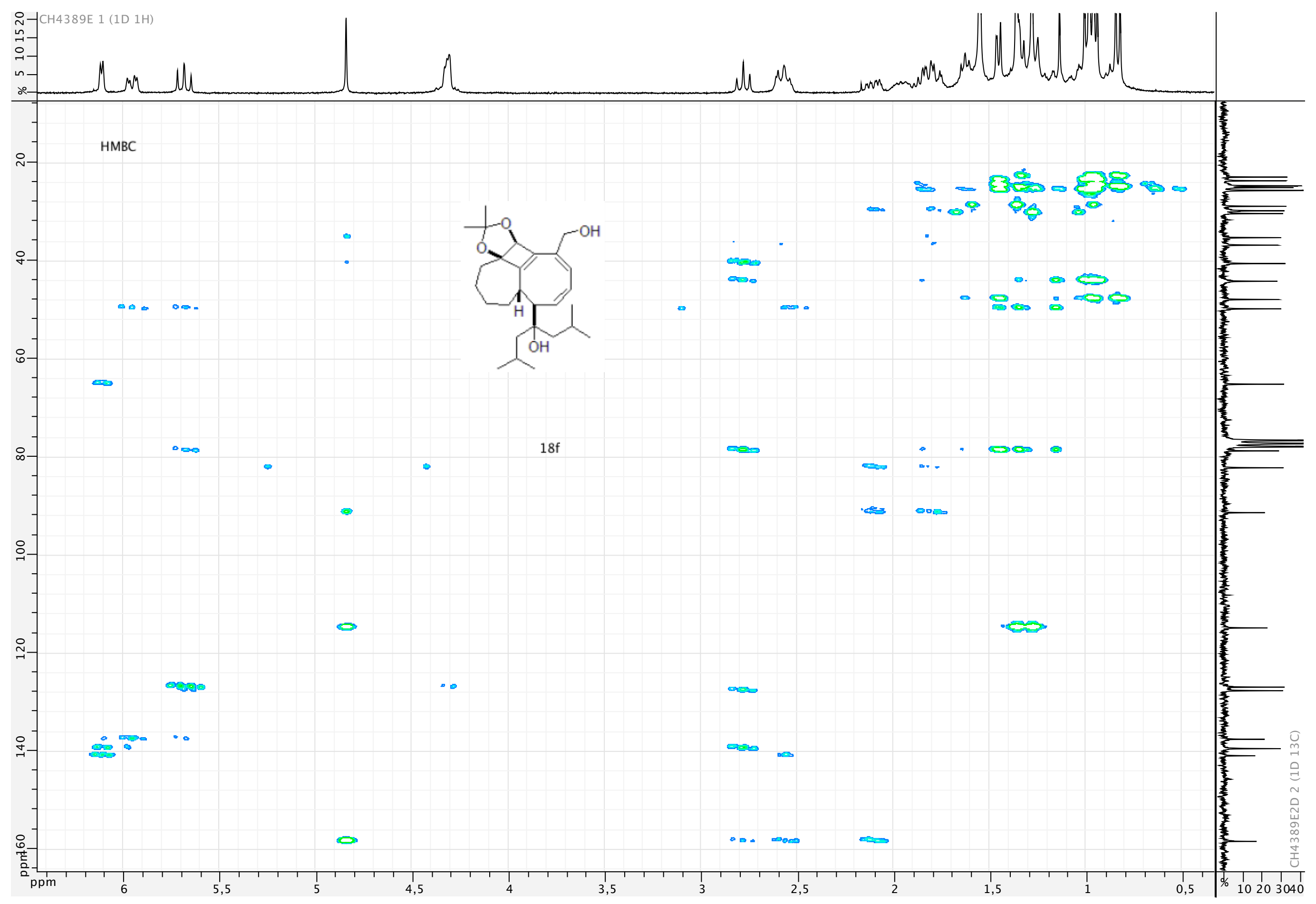

